\title{
Cellulose based materials as a template for the synthesis of copper nanoparticles for antimicrobial applications
}

\author{
Tuhua Zhong
}

Follow this and additional works at: https://researchrepository.wvu.edu/etd

\section{Recommended Citation}

Zhong, Tuhua, "Cellulose based materials as a template for the synthesis of copper nanoparticles for antimicrobial applications" (2015). Graduate Theses, Dissertations, and Problem Reports. 7046.

https://researchrepository.wvu.edu/etd/7046

This Dissertation is protected by copyright and/or related rights. It has been brought to you by the The Research Repository @ WVU with permission from the rights-holder(s). You are free to use this Dissertation in any way that is permitted by the copyright and related rights legislation that applies to your use. For other uses you must obtain permission from the rights-holder(s) directly, unless additional rights are indicated by a Creative Commons license in the record and/ or on the work itself. This Dissertation has been accepted for inclusion in WVU Graduate Theses, Dissertations, and Problem Reports collection by an authorized administrator of The Research Repository @ WVU.

For more information, please contact researchrepository@mail.wvu.edu. 


\title{
CELLULOSE BASED MATERIALS AS A TEMPLATE FOR THE SYNTHESIS OF COPPER NANOPARTICLES FOR ANTIMICROBIAL APPLICATIONS
}

\author{
Tuhua Zhong \\ Dissertation submitted \\ to the Davis College of Agriculture, Natural Resources and Design \\ at West Virginia University \\ in partial fulfillment of the requirements for the degree of \\ Doctor of Philosophy in \\ Forest Resources Science
}

Gloria S. Oporto, Ph.D., Committee Chair

Benjamin Dawson-Andoh, Ph.D.

Jacek Jaczynski, Ph.D.

Robin Hissam, Ph.D.

Xiaodong Shi, Ph.D.

School of Natural Resources

Morgantown, West Virginia

2015

Keywords: cellulose, nanocellulose, copper nanoparticles, thermoplastic resins, antimicrobial, thermal properties, mechanical properties

Copyright 2015 Tuhua Zhong 


\section{ABSTRACT \\ CELLULOSE BASED MATERIALS AS A TEMPLATE FOR THE SYNTHESIS OF COPPER NANOPARTICLES FOR ANTIMICROBIAL APPLICATIONS}

\section{Tuhua Zhong}

Copper has been used as a broad-spectrum biocide for centuries, showing effective antimicrobial performance against bacteria, fungi and viruses. Among various forms of copper, copper nanoparticles is attracting particular attention because of its high surface area and crystal morphology that will enhance copper antimicrobial properties. However, the high tendency of copper particles to aggregate at a nanoscale remains as a drawback for the development of copper-based nanostructured products. In this research, cellulose-based materials were utilized as a template and stabilizer for copper nanoparticles, and the resulting hybrid material was used as bi-functional material in thermoplastic resins improving the final antimicrobial and mechanical performance of the composites. This research work was divided in the following stages: 1) developing of a simple way to synthesize copper nanoparticles using cellulose raw material as template and stabilizer for copper; 2) evaluating the antimicrobial, thermal, mechanical, and copper release performance of films prepared by adding the hybrid cellulose-copper nanoparticles into a thermoplastic resin using a wet process; 3) investigating and characterizing composite materials after the incorporation of hybrids cellulose-copper nanoparticles on thermoplastic resins using a extrusion/film formation process (dry process); 4) evaluating drying methodologies to dry hybrid cellulose-copper nanoparticles suspensions determining their morphology, particle size, oxidation state of copper and copper crystallite size. 


\section{DEDICATION}

I would like to dedicate this dissertation to my parents for their endless understanding, love and support throughout my life. 


\section{ACKNOWLEDGMENTS}

I would like to express my sincere gratitude to my advisor and chair of my graduate committee, Dr. Gloria S. Oporto, for helping me develop this research plan, for giving constant feedback on the results and drafts I passed along to her, and especially for guiding me as I have grown as a researcher and an academic over the past four years. Without Dr. Oporto's unfailing supports and instructions, I could not have completed my doctoral program.

I would like to thank my graduate committee Dr. Jacek Jaczynski, for his patience with me discussing the antimicrobial testing experiments and helping revise my manuscripts that have been published in the peer-review journals.

I would like to thank my other members serving in this graduate committee, Dr. Ben Dawson-Andoh, Dr. Robin Hissam, Dr. Xiaodong Shi for spending the time evaluating my work and providing constructive suggestions and feedback.

I appreciate my thanks to USDA Forest Service, Forest Products Laboratory where I was allowed to use the twin-screw extruder to prepare my samples, and Dr. Ronal Sabo for his kind offer of TEMPO nanofibrillated cellulose.

Many thanks go to WVU shared research facilities staff, Dr. Weiqiang Ding for his help with XRD and XPS analyses, and Dr. Marcela Redigolo for her help with SEM and TEM analyses.

I would like to thank Dr. Xinfeng Xie, a Postdoctoral researcher, for his patience with me discussing the XRD analysis and helping revise my manuscript.

It has been my pleasure to work with all members-Juan Carrasco, Changle Jiang, Masoumeh Hassanzadeh in Dr. Gloria Oporto's group past and present for their help. 
I acknowledge the funding for this work that was provided by NIFA McStennis WVA00098 "Efficient utilization of biomass for biopolymers in central Appalachia", and USDA NIFA Grant No. 2013-34638-21481 "Development of novel hybrid cellulose nanocomposite film with potent biocide properties utilizing low quality Appalachian hardwoods". 


\section{TABLE OF CONTENTS}

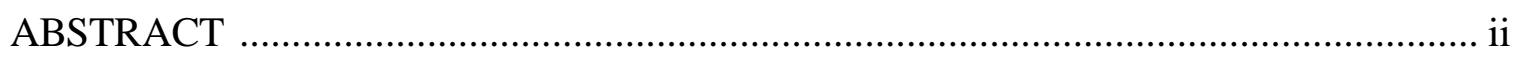

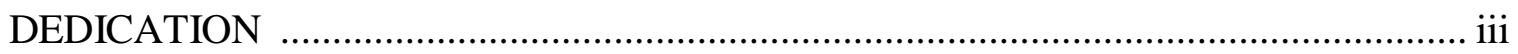

ACKNOWLEDGMENTS ………………………………........................................iv

TABLE OF CONTENTS .....................................................................................

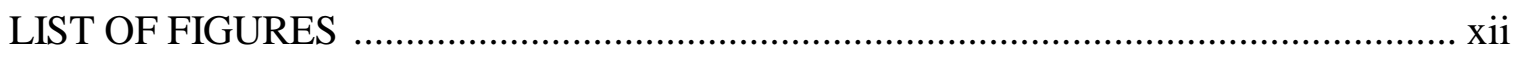

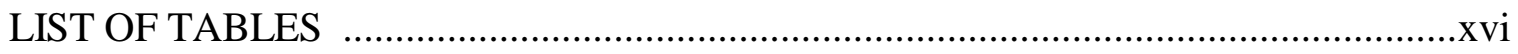

LIST OF SYMBOLS/ABREVIATION/NOMENCLATURE ......................................... vii

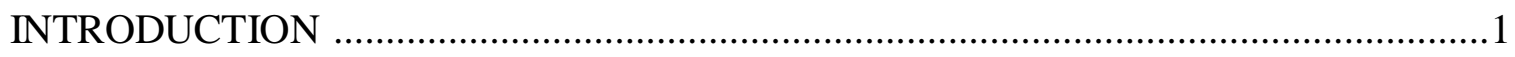

CHAPTER 1: LITERATURE REVIEW ..........................................................10

1.1 Antimicrobial food packaging ………………………...................................10

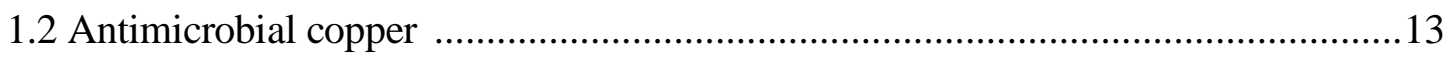

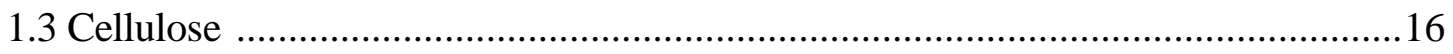

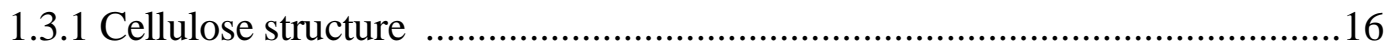

1.3.2 Carboxylate cellulose as template for copper nanoparticles ..........................17

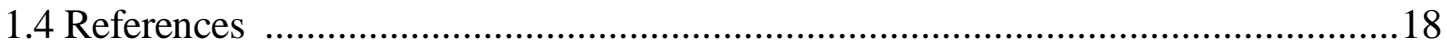

CHAPTER 2: ANTIMICROBIAL PROPERTIES OF THE HYBRID COPPER NANOPARTICLES-CARBOXYMETHYL CELLULOSE $\quad$..........27

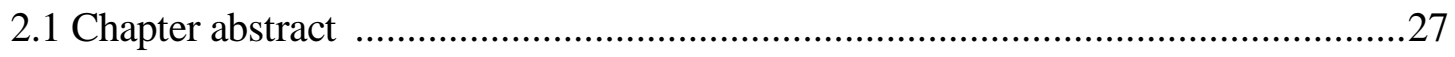

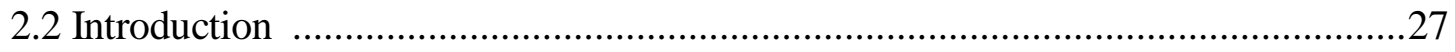

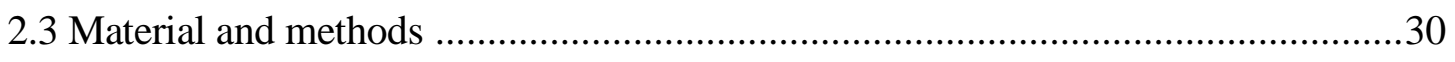

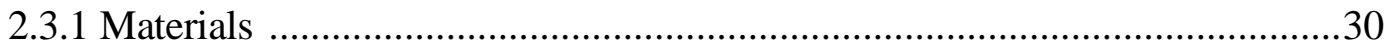

2.3.2 Preparation of copper-carboxymethyl cellulose composite material ..............31

2.3.3 Scanning electron microscopy-energy-dispersive X-ray spectroscopy characterization $\quad$..................31

2.3.4 Transmission electron microscopy characterization .......................................31

2.3.5 Antimicrobial properties of copper-carboxymethyl cellulose solution against $\begin{array}{llll}\text { nonpathogenic } \quad \text { Escherichia } & \text { coli }\end{array}$ 
2.4 Results and discussion

2.4.1 Scanning electron microscopy-energy-dispersive X-ray spectroscopy characterization of pure carboxymethyl cellulose and carboxymethyl cellulosecopper

nanoparticle

films 33

2.4.2 Transmission electron microscopy analysis of carboxymethyl cellulosecopper nanoparticles

solution 36

2.4.3 Antimicrobial properties of copper-carboxymethyl cellulose solution against nonpathogenic Escherichia coli

DH5 $\alpha$ 36

2.5 Conclusions .38

2.6 References .38 CHAPTER 3: NANOFIBRILLATED CELLULOSE AND COPPER NANOPARTICLES EMBEDDED IN POLYVINYL ALCOHOL FILMS FOR ANTIMICROBIAL APPLICATION .43

3.1 Chapter abstract .43

3.2 Introduction .43

3.3 Materials and methods .45

3.3.1 Materials .45

3.3.2 Preparation of the hybrid TNFC-copper nanoparticles .46

3.3.3 Preparation of PVA/TNFC and PVA/TNFC-copper nanoparticles nanocomposite

films 47

3.3.4 Transmission

electron microscopy

(TEM)

3.3.5 Thermogravimetric

analyzer

(TGA)

characterization .48

3.3.6 Determination of antimicrobial activity of PVA/TNFC-copper nanoparticles nanocomposite

films 48 
3.4 Results and discussion

3.4.1 Morphology of copper nanoparticles on TNFC template

3.4.3 Antimicrobial activity of PVA/TNFC-copper nanoparticles films

vii

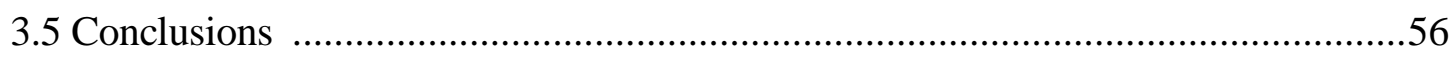

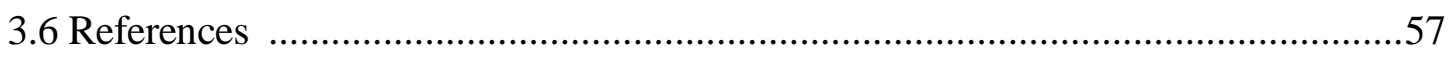

CHAPTER 4: USING TWO CELLULOSIC TEMPLATES TO SYNTHESIZE COPPER NANOPARTICLES FOR ANTIMICROBIAL PURPOSES ........................................60

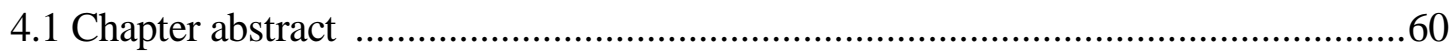

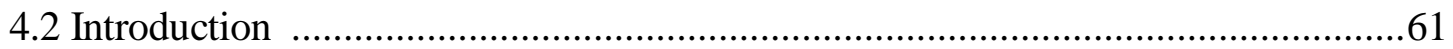

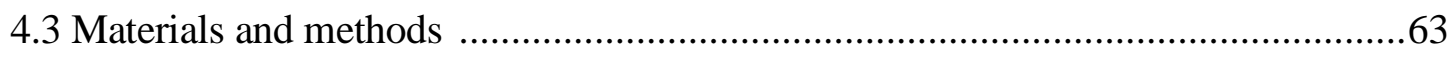

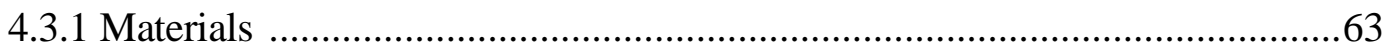

4.3.2 Preparation of the hybrid TNFC-copper nanoparticles ..............................64

4.3.3 Preparation of the hybrid CMC-copper nanoparticles ...............................64

4.3.4 Preparation of PVA/TNFC-copper nanoparticles and PVA/CMC-copper

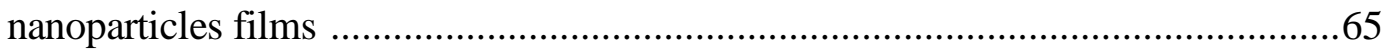

4.3.5 Transmission electron microscopy (TEM) ..........................................66

4.3.6 Ultraviolet-visible (UV-vis) characterization ..........................................66

4.3.7 X-ray diffraction (XRD) characterization ................................................66

4.3.8 X-ray photoelectron spectroscopy (XPS) characterization .........................67

4.3.9 Antimicrobial activities of PVA/TNFC-Cu and PVA/CMC-Cu films .........67

4.3.10 Inductively coupled plasma-optical emission (ICP-OES) spectrometry ......68

4.3.11 Thermo-gravimetric analyzer (TGA) characterization .............................69

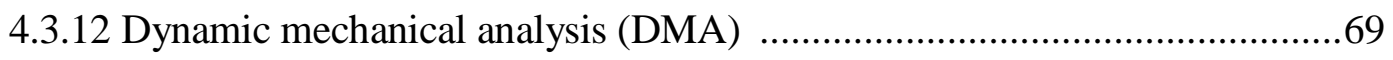

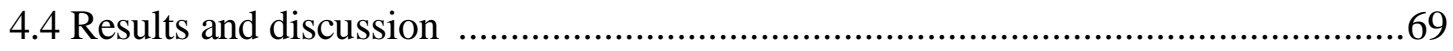

4.4.1 Morphological characterization of the hybrid TNFC-copper nanoparticles and CMC-copper nanoparticles 
4.4.2 Ultraviolet-visible (UV-vis) analysis

.70

4.4.3 Copper crystal structure and oxidation states of copper within PVA/TNFC-

$\mathrm{Cu} 0.6$ and PVA/CMC-Cu0.6 films .71

4.4.4 Antimicrobial activities of PVA/TNFC-Cu0.6 and PVA/CMC-Cu0.6 films .75

4.4.5 Copper ion release from PVA/cellulose-copper nanoparticles films .78

4.4.6 Thermal analysis .81

4.4.7 Dynamic mechanical analysis .83

4.5 Conclusions .86

4.6 References .87

CHAPTER 5: MICROSTRUCTURE, MECHANICAL, THERMAL AND ANTIMICROBIAL PROPERTIES OF FREEZE-DRIED HYBRID COPPER NANOPARTICLES AND CELLULOSE BASED MATERIALS EMBEDDED IN THERMOPLASTIC RESINS .94

5.1 Chapter abstract .94

5.2 Introduction .95

5.3 Materials and methods .99

5.3.1 Materials .99

5.3.2 Preparation of the hybrid CMC-copper nanoparticles .......100 5.3.3 Preparation of the hybrid TNFC-copper nanoparticles .......101 5.3.4 Preparation of composites: hybrid cellulose-copper nanoparticles embedded in thermoplastic

resins 101

5.3.5 Field emission scanning electron microscopy-energy dispersive X-ray spectroscopy characterization

(FESEM-EDX) 103

5.3.6 Mechanical strength-tensile testing 103

5.3.7 Differential scanning calorimetry (DSC) characterization 104 5.3.8 Atomic absorption spectroscopy (AAS) characterization of copper ion release from thermoplastic composites in an aqueous medium 105 
5.3.9 Antimicrobial properties of composite film of PLA containing freeze-dried hybrid 105

5.4 Results and discussion 106

5.4.1 Morphological and copper elemental mapping analysis of the hybrid cellulose-copper nanoparticles 106

5.4.2 Microstructure of the hybrids CMC-copper nanoparticles and TNFC-copper nanoparticles embedded in thermoplastic resins (extruded-injected specimens)... 107 5.4.3 Mechanical strength-tensile testing on the hybrids CMC-copper nanoparticles and TNFC-copper nanoparticles embedded in thermoplastic resins (extrudedinjected specimens) 110

5.4.4 Thermal properties of hybrid copper nanoparticles and cellulose based materials in thermoplastic resins (extruded-injected specimens) .112 5.4.5 Copper ion release from extruded-injected composites and film composites in aqueous medium

5.4.6 Antimicrobial properties of pure PLA film and PLA/TNFC-Cu film against E.coli DH5 $\alpha$

5.5 Conclusions 118

5.6 References 119

CHAPTER 6: DRYING CELLULOSE-BASED MATERIALS CONTAINING COPPER NANOPARTICLES

6.1 Chapter abstract

6.2 Introduction 128

6.3 Materials and methods 130

6.3.1 Materials 130

6.3.2 Preparation of control suspensions of cellulose base materials: CMC and TNFC 131

6.3.3 Preparation of the hybrid CMC-copper nanoparticles 131 
6.3.4 Preparation of the hybrid TNFC-copper nanoparticles

6.3.5 Spray drying process

6.3.6 Freeze drying process

6.3.7 Modified freeze drying process

6.3.8 Field emission scanning electron microscopy-energy dispersive X-ray spectroscopy characterization (FESEM-EDX)

6.3.9 Laser diffraction (LD) characterization

6.3.10 Inductively coupled plasma-optical emission spectroscopy (ICP-OES) characterization

6.3.11 X-ray photoelectron spectroscopy (XPS) characterization

6.3.12 X-ray diffraction (XRD) characterization

6.4 Results and discussion

6.4.1 Morphology of the spray-, freeze- and modified freeze dried cellulosic materials

6.4.2 Particle size distribution using laser diffraction (LD)

6.4.3 Energy dispersive X-ray (EDX) elemental quantification and ICP-OES analysis to determine copper content on the dried materials

6.4.4 Oxidation state of copper CMC-copper nanoparticles and TNFC-copper nanoparticles using X-ray photoelectron spectroscopy (XPS)

6.4.5 Crystal structures of CMC-copper nanoparticles and TNFC-copper nanoparticles using X-ray diffraction (XRD)

6.4.6 Estimation of crystallite size using XRD data.....

6.5 Conclusions

6.6 References 155

CHAPTER 7: CONCLUSIONS AND REMARKS ON FUTURE RESEARCH 160

7.1 Conclusions 160

7.2 Remarks on future research 163

7.3 References 165 


\section{LIST OF FIGURES}

Figure 1. 1 Antimicrobial packaging systems (Han 2000; Quintavalla and Vicini 2002; Bastarrachea et al. 2011)

Figure 2. 1 (a) Scanning electron microscope (SEM) image of pure carboxymethyl cellulose $(\mathrm{CMC})$ film (scale bar $=1 \mu \mathrm{m})$; (b) SEM image of CMC-copper particles film (scale bar $=1 \mu \mathrm{m})$.

Figure 2. 2 Scanning electron microscope (SEM) image of "big" spherical particles present on the surface of the hybrid carboxymethyl cellulose-copper film (scale bar $=6$ $\mu \mathrm{m})$. Spectrum 1 shows the place of energy-dispersive X-ray spectroscopy analysis (Table 2.1)

Figure 2. 3 Scanning electron microscope image of a "small" spherical particle present on the surface of the hybrid carboxymethyl cellulose-copper nanoparticles film. Spectrum 1 shows the place of energy-dispersive X-ray spectroscopy analysis (Table 2.2) .35

Figure 2. 4 (a) Transmission electron microscopy (TEM) image of copper particles on the carboxymethyl cellulose (CMC) structure (b) high-resolution TEM image of copper particles on the CMC structure .36

Figure 2. 5 Bacterial enumeration of the 18-h Escherichia coli culture. (a) Representative Petrifilm plate for $10^{-6}$ dilution with too-numerous-to-count number of colonies; (b) representative Petrifilm for $10^{-7}$ dilution with 34 colonies. Thus, the 18 -h E. coli culture contained $3.4 \times 10^{8}$ colony-forming units $/ \mathrm{mL}$

Figure 2. 6 Bacterial enumeration of the 18-h Escherichia coli culture exposed to coppercarboxymethyl cellulose solution for $10 \mathrm{~h}$ at 4 . (a) Representative Petrifilm plate ${ }^{\circ} \mathrm{C}$ for $10^{-1}$ dilution. (b) Representative Petrifilm plate for $10^{-2}$ dilution ......................38

Figure 3. 1 (a) TEM images of hybrid TNFC-copper nanoparticles (Scale bar = $10 \mathrm{~nm}$ ); (b) histogram of particle size distribution ( $n=55$ particles); (c) EDX spectrum of the hybrid TNFC-copper nanoparticles.

Figure 3. 2 (a) TGA curves for the pure PVA, PVA/TNFC film, and PVA/TNFC-Cu composite films; (b) the corresponding DTG curves. Peak temperatures $\left(T_{\max }\right)$ of 
PVA, PVA/TNFC, PVA/TNFC-Cu0.4, PVA/TNFC-Cu0.5, PVA/TNFC-Cu0.6, and PVA/TNFC-Cu0.7 were 260, 263, 274, 275, 278, and 278 , respectively). .....51 ${ }^{\circ} \mathrm{C}$

Figure 3. 3 Bacterial enumeration of the 24-h Escherichia coli culture exposed to control materials (a, b) PVA film and (c, d) PVA/TNFC film for 1 week at room temperature. ( $\mathrm{a}, \mathrm{c}$ ) Representative Petrifilm plates for $10^{-5}$ dilution; (b, d) Representative Petrifilm co unt plates for $10^{-6}$ dilution

Figure 3. 4 Bacterial enumeration of the 24-h Escherichia coli culture exposed to PVA nanocomposite film containing various copper content (a, b) PVA/TNFC-Cu0.4, (c, d) PVA/TNFC-Cu0.5, and (e, f) PVA/TNFC-Cu0.6 films for 1 week at room temperature. (a-f) Representative Petrifilm count plates for various dilutions 55

Figure 3. 5 Microbial reduction induced by PVA/TNFC-copper nanoparticles after 1-week exposure. The Asterisks refer to significant levels compared to one of the controls: PVA/TNFC, $\quad \mathrm{p}<0.05 \quad(*), \quad \mathrm{p}<0.01 \quad(* *), \quad$ and $\quad \mathrm{p}<0.001(* * *)$. .56

Figure 4. 1 TEM images of (a) TNFC-copper nanoparticles (scale bar $=10 \mathrm{~nm}$ ) and (b)

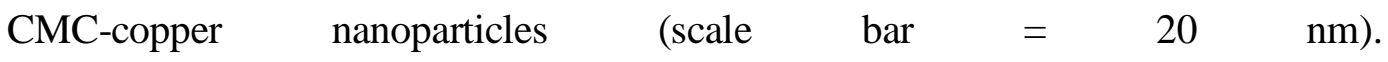

Figure 4. 2 UV-vis spectra of carboxylate cellulose suspensions and hybrid cellulosecopper nanoparticles suspensions .71

Figure 4. 3 XRD patterns of PVA composite films .73

Figure 4. 4 XPS full survey scan of (a) PVA/TNFC-Cu0.6 and (b) PVA/CMC-Cu0.6. Inset: $\begin{array}{lllll}\mathrm{Cu} 2 \mathrm{p}_{3 / 2} & \text { and } & \mathrm{Cu} 2 \mathrm{p}_{1 / 2} & \mathrm{XPS} & \text { detail scan }\end{array}$

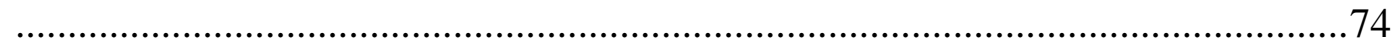

Figure 4. 5 Bacterial enumeration of the 24-h Escherichia coli culture exposed to PVA/TNFC-Cu0.6 film (a,c,e) and PVA/CMC-Cu0.6 film (b,d,f) after various exposure time .77

Figure 4. 6 Microbial reduction induced by PVA containing cellulose-copper nanoparticles after various exposure time 
Figure 4. 7 The amount of copper leaching from PVA/TNFC-Cu0.6 and PVA/CMC-Cu0.6 films and their corresponding mass fraction of copper ions leaching from the films (inset) .

Figure 4.8 (a) TGA curves for the pure PVA, PVA/TNFC film, and PVA/TNFC-Cu0.6 film, PVA/CMC film, and PVA/CMC-Cu0.6 film; (b) the corresponding DTG 
curves (Peak temperature $\left(T_{\max }\right)$ of PVA, PVA/TNFC, PVA/TNFC-Cu0.6, PVA/CMC, PVA/CMC-Cu0.6 was 260, 263, 278, 269, and 285, respectively); ${ }^{\circ} \mathrm{C}$

(c) TGA cure for CMC and CMC-copper nanoparticles and its corresponding DTG cure (Inset)

Figure 4. 9 (a) Storage modulus as a function of temperature for PVA composite films and

(b) corresponding tan

Figure 5. 1 FESEM-EDX characterization of (a) hybrid CMC-copper nanoparticles and (b) hybrid TNFC-copper nanoparticles and their corresponding copper elemental mapping images of (c) hybrid CMC-copper nanoparticles and (d) hybrid TNFCcopper nanoparticles (Tiny light dots represent copper element) 107

Figure 5. 2 SEM images of cryo-fractured surfaces of (a) PP, (b) PP/CMC-Cu (1), (c) PP/CMC-Cu (2), (d) PP/CMC-Cu (3), (e) PP/CMC-Cu (4), (f) PP/TNFC-Cu ......108

Figure 5. 3 SEM images of cryo-fractured surfaces of (a) PLA, (b) PLA/CMC-Cu (1), (c) PLA/CMC-Cu (2), (d) PLA/CMC-Cu (3), (e) PLA/CMC-Cu (4), (f) PLA/TNFC$\mathrm{Cu}$ 109

Figure 5. 4 SEM images of cryo-fractured surfaces of (a) PLA, (b) PLA/CMC-Cu(3), (c) PLA/CMC-Cu(4), (d) PLA/TNFC-Cu at higher magnification. 110

Figure 5. 5 Tensile strength of (a) PP composites and (b) PLA composites .111

Figure 5. 6 DSC analysis for (a) PP and PP composites, and (b) PLA and PLA composites.

Figure 5. 7 Degree of crystallinity of (a) PP composites and (b) PLA composites .116

Figure 6. 1 SEM images of dried materials. CMC: a) spray dried, b) freeze dried; CMCcopper nanoparticles: c) spray dried, d) freeze dried; TNFC: e) spray dried, f) freeze dried. 137

Figure 6. 2 SEM images of freeze and modified freeze dried materials: CMC: (a) CMC freeze dried, (b) CMC after an E/1-B-FD treatment; CMC-Cu: (c) CMC-Cu freeze dried, (d) CMC-Cu after an E/1-B-FD treatment; TNFC: (e) TNFC freeze dried, (f) TNFC after an E/1-B-FD treatment; TNFC-Cu: (g) TNFC-Cu freeze dried, (h) TNFC-Cu after an E/1-B-FD treatment. 
Figure 6. 3 SEM images of freeze and modified freeze dried materials: CMC: (a) CMC freeze dried, (b) CMC after an E/tert-B-FD treatment; CMC-Cu: (c) CMC freeze dried, (d) CMC-Cu after an E/tert-B-FD treatment; TNFC: (e) TNFC freeze dried, (f) TNFC after an E/tert-B-FD treatment; TNFC-Cu: (g) TNFC-Cu freeze dried, (h) TNFC-Cu after an E/tert-B-FD treatment

Figure 6. 4 Particle size distribution of spray dried particles: (a) CMC, (b) CMC-copper nanoparticles, (c) TNFC

Figure 6. 5 Average diameter size histograms at 10, 50 and 90 percentile for various spray dried particles

Figure 6. 6 (a) Energy dispersive X-Ray (EDX) copper elemental quantification of dried CMC-copper nanoparticles materials, (b) ICP-OES quantification of copper contents on CMC and TNFC templates

Figure 6. 7 XPS full survey scan of (a) SD CMC-copper nanoparticles, (b) FD CMCcopper nanoparticles and (c) E/1-B-FD CMC-copper nanoparticles. Inset: $\mathrm{Cu} 2 \mathrm{p}_{3 / 2}$ and $\mathrm{Cu} 2 \mathrm{p}_{1 / 2}$ XPS detail scan

Figure 6. 8 XPS full survey scan of (a) FD TNFC-copper nanoparticles, and (c) E/tert-BFD TNFC-copper nanoparticles. Inset: $\mathrm{Cu} 2 \mathrm{p}_{3 / 2}$ and $\mathrm{Cu} 2 \mathrm{p}_{1 / 2}$ XPS detail scan ........148

Figure 6. 9 XRD patterns of (a) CMC based materials obtained from SD process, (b) CMC based materials obtained FD and E/1-B-FD processes and (c) TNFC based materials obtained from FD and E/tert-B-FD processes 151 


\section{LIST OF TABLES}

Table 2. 1 Energy-dispersive X-ray spectroscopy (EDX) analysis on the spherical 'big' particles present on the surface of carboxymethyl cellulose-copper nanoparticle films (Figure 2.2)

Table 2. 2 Energy-dispersive X-ray spectroscopy (EDX) analysis on the spherical 'small' particles present on the surface of carboxymethyl cellulose-copper nanoparticle

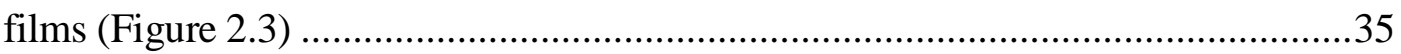

Table 3. 1 Preparation of the hybrid TNFC-copper nanoparticles ...................................47

Table 3. 2 Preparation of the PVA/TNFC-copper nanoparticles nanocomposite films........48

Table 4. 1 Weight percentage (wt.\%) of main element to the total weight of PVA, cellulose and copper

Table 4. 2 Binding energy $\left(\mathrm{Cu} 2 \mathrm{p}_{3 / 2}\right)$ for copper different state of oxidation (McIntyre and Cook 1975)

Table 5. 1 Preparation of the hybrid CMC-copper nanoparticles

Table 5. 2 Processing setting parameters for PP and PLA extruded-injected composites. 102

Table 5. 3 Mass percentage of the thermoplastic resin PP and the hybrid cellulose-copper nanoparticles in the composite production 102

Table 5. 4 Mass percentage of the thermoplastic resin PLA and the hybrid cellulose-copper nanoparticles in the composite production 103

Table 5. 5 Copper ions released from PLA film composites 117

Table 5. 6 Antimicrobial results for PLA film and PLA/TNFC-Cu film against E.coli DH5 $\alpha$ under different exposure time.

Table 6. 1 Drying experiments

Table 6. 2 Binding energy $\left(\mathrm{Cu} 2 \mathrm{p}_{3 / 2}\right)$ for copper different states of oxidation (Mclntyre and Cook 1975) 148

Table 6. 3 Summary of binding energy for $\mathrm{Cu} 2 \mathrm{p}_{3 / 2}$

Table 6. 4 Typical XRD parameters for copper and cellulose according to its chemical state (International Center for Diffraction Data (ICDD) 


\section{LIST OF SYMBOLS/ABREVIATION/NOMENCLATURE}

CMC: Carboxymethyl cellulose

DMA: Dynamic mechanical analysis

DSC: Differential scanning calorimetry

EDX: Energy-dispersive X-ray spectroscopy

ICP-OES: Inductively coupled plasma-optical emission spectroscopy

LD: Laser diffraction

LTB: Lauryl tryptose broth

PLA: Polylactic acid

PP: Polypropylene

PVA: Polyvinyl alcohol

SEM: Scanning electron microscopy

TEM: Transmission electron microscopy

TEMPO: 2,2,6,6-tetramethylpiperidine-1-oxyl radical

TGA: Thermogravimetric analyzer

TNFC: TEMPO nanofibrillated cellulose

TSB: Trypticase soy broth

UV-vis: Ultraviolet-visible spectroscopy

XPS: X-ray photoelectron spectroscopy

XRD: X-ray diffraction 


\section{INTRODUCTION}

Motivation. The flexible packaging industry represents the second largest production in the packaging industry, which is approximately $\$ 135$ billion around the world (USA Flexible Packaging Association). Any improvement in the manufacture of these films will generate benefits not only to the industry but also to the population. The development of antimicrobial packaging, with the aim to inhibit the growth of microorganisms on the surface of the food, continues to be developed in response to the increasing demand for extended shelf life and food safety (Han 2003; Suppakul et al. 2003). US Center for Disease Control and Prevention estimated that there are approximate 76 million foodrelated illnesses resulting in 325,000 hospitalizations and 5,000 deaths in the United States each year (Mead et al. 1999). Foodborne pathogens are still the leading causes of illness and death, resulting in billions of dollars loss in medical care associated with foodborne illness. Therefore, the emerging of antimicrobial film would open a new avenue for combating pathogen and preventing foodborne illness.

Hypothesis. Our main hypothesis is that functionalized cellulose with carboxylic groups can be effectively used as a template and stabilizer for the synthesis of the antimicrobial agent copper in the form of nanoparticles. The incorporation of the hybrid cellulose/copper nanoparticles into thermoplastic resins intended for packaging film application will provide them with antimicrobial properties against foodborne microorganisms.

Overall research goal and specific objectives. The overall goal of this research was to develop an hybrid cellulose-copper nanoparticles material with antimicrobial properties for packaging film applications. 


\section{Specific objectives:}

1) To develop a simple and effective methodology to synthesize "in situ" copper nanoparticles on the cellulosic raw materials composed of carboxymethyl cellulose (CMC) and TEMPO nanofibrillated cellulose (TNFC).

2) To evaluate the antimicrobial, thermal and mechanical performance of thermoplastic films after the incorporation of the hybrid cellulose-copper nanoparticles using a wet process.

3) To evaluate the antimicrobial, thermal and mechanical performance of thermoplastic films after the incorporation of the hybrid cellulose-copper nanoparticles using a dry process.

4) To evaluate different drying methods to dry the hybrid cellulose-copper nanoparticle suspensions and investigate changes in their morphology, particle size, copper content and oxidation state of copper. 
Research approach. To accomplish the specific objectives the following approaches were made:

Copper nanoparticles were in situ introduced to cellulosic templates through chemical reduction method in an aqueous system. The surface morphology of cellulose/copper nanoparticles was observed under scanning electron microscopy (SEM). Energy-dispersive $\mathrm{X}$-ray spectroscopy (EDX) was used in this study to characterize the chemical composition of the hybrid cellulose-copper nanoparticles. High-resolution transmission electron microscopy (HRTEM) was used to determine the size, shape and size distribution of copper nanoparticles on the cellulosic templates. Antimicrobial activities against E.coli DH5a, following exposure to the hybrid cellulose-copper nanoparticles suspension, have been studies using dynamic shake flask test followed by plate counting method.

The incorporation of the hybrid cellulose-copper nanoparticles into the polar thermoplastic matrix, polyvinyl alcohol (PVA), was performed through a solvent casting method in a wet process. Antimicrobial activities against E.coli $\mathrm{DH} 5 \alpha$, following exposure to the thermoplastic films, were evaluated using a plate counting method. Copper ion release from the films was determined by inductively coupled plasma-optical emission spectroscopy (ICP-OES). X-ray photoelectron spectroscopy (XPS) was used to characterize the film surface chemistry and determine the state of oxidation of copper. The particular crystalline structure and the chemical composition of the copper nanoparticles within the films were investigated under X-ray diffraction (XRD). Thermogravimetric analysis (TGA) was used in this study to evaluate the film thermal properties after the incorporation of hybrid cellulose-copper nanoparticles. The film mechanical performance was evaluated using dynamical mechanical analysis (DMA).

The incorporation of the hybrid cellulose-copper nanoparticles into non-polar thermoplastic matrices such as polypropylene (PP) and polylactic acid (PLA) was performed using a drying process. The hybrid cellulose-copper nanoparticles were incorporated into the thermoplastic matrix using a twin-screw extruder followed by both injection molding 
process and a dry film formation process. Antimicrobial activities against E.coli DH5 following exposure to the thermoplastic films, were evaluated using plate counting method. The microstructure of the film after the incorporation of hybrid materials was observed using SEM. The thermal properties was evaluated by differential scanning calorimetry (DSC). The film mechanical performance was evaluated using tensile strength testing according to ASTM D638 standard. The copper ion release properties of thermoplastic composites were determined using atomic absorption spectroscopy (AAS).

Finally, with the aim to improve the incorporation of the hybrid material in a dry process three drying methods were examined to dry the hybrid cellulose-copper nanoparticles suspensions. The drying methods studied were: (1) freeze-drying; (2) spray-drying; (3) modified freeze-drying. The morphologies of dried hybrid materials were observed by SEM. Laser diffraction (LD) analysis was the technique used to determine the size and size distribution of spray dried particles. Copper content on the cellulosic templates before and after the various drying processes were determined by inductively coupled plasma-optical emission spectroscopy (ICP-OES). The state of oxidation of copper on the cellulosic templates was analyzed using XPS. The particular crystalline structure, crystallite size and the chemical composition were analyzed by XRD.

Dissertation organization. This dissertation consists of seven chapters. In chapter 1 is presented a background on the antimicrobial packaging film area, copper as a potent biocide and cellulose based materials as template for biocide materials. Chapter 2-5 correspond to the main research performed and have been written based on three peerreview papers published during the development of this dissertation research. Chapter 6 corresponds to a research published in a conference proceedings and in Chapter 7 is presented the general conclusions of this dissertation research and proposed future work.

In Chapter 1 is presented background on the antimicrobial packaging film area, copper as a potent biocide and cellulose based materials as template for biocide materials. 
In Chapter 2 a simple method to introduce copper nanoparticles on carboxymethyl cellulose template using sodium borohydride as a copper reducing agent is presented. Copper nanoparticles with the diameter of 10 - to $20 \mathrm{~nm}$ were observed on the cellulose surface. Antimicrobial test exhibited that the hybrid carboxymethyl cellulose-copper nanoparticles (CMC-copper nanoparticles) have effective antimicrobial activity against E.coli $\mathrm{DH} 5 \alpha$, a nonpathogenic surrogate of foodborne pathogen.

In Chapter $\mathbf{3}$ is presented the antimicrobial properties of the hybrid TEMPO nanofibrillated cellulose (TNFC)-copper nanoparticles embedded in a polar thermoplastic resin (Polyvinyl alcohol). Likewise, the investigation of the effect of copper concentration on the antimicrobial and thermal properties of PVA composite films was performed. The thermal properties were characterized using TGA and the antimicrobial properties were tested against E.coli DH5 $\alpha$. The PVA composite films containing various copper content were prepared by solvent casting of the mixture of PVA solution and TNFC-copper nanoparticle suspension. The results indicated that increasing copper content in the PVA films resulted in greater reduction of E.coli DH5 $\alpha$, the optimum copper loading is $0.6 \mathrm{wt} . \%$ that could result in up to 5-log microbial E.coli reduction after 1-week exposure.

In Chapter 4 is presented the correlation between copper ion release and antimicrobial properties of PVA composite films, and the probable antimicrobial mechanism of PVA composite films. PVA composite films containing the same copper loading $(0.6 \mathrm{wt} . \%)$ with different cellulosic templates (CMC and TNFC) were prepared, respectively. Approximately 90\% less cellulosic material was required when using TNFC as copper template compared to CMC template to get similar films antimicrobial performance. Antimicrobial and copper ion release studies indicated that there is no direct evidence showing correlation between antimicrobial properties and the amount of copper ion release. PVA embedded with CMC-copper nanoparticles inactivated more E.coli DH5 $\alpha$ at the same exposure time compared with PVA containing TNFC-copper nanoparticles. That might be attributed to higher amount of crystalline $\mathrm{Cu}_{2} \mathrm{O}$ in PVA/CMC matrix. The decomposition temperatures, corresponding to maximum weight loss of PVA films, were increased by 18 
${ }^{\circ} \mathrm{C}$ and $25{ }^{\circ} \mathrm{C}$ when TNFC-copper nanoparticles and CMC-copper nanoparticles were incorporated into the films, respectively. Likely, the storage modulus at $48{ }^{\circ} \mathrm{C}$ was also enhanced by $164 \%$ and $217 \%$, respectively.

In Chapter 5 is presented a preliminary research on the incorporation of freeze-dried hybrid materials into nonpolar and hydrophobic thermoplastic resins [polypropylene (PP) and polylactic acid (PLA)]. Composites were prepared using a twin-screw extruder followed by both injection molding process and a dry film formation process. The results indicated that the incorporation of freeze-dried hybrid materials into PP and PLA has negative effects on the tensile strength due to the aggregates of hybrid materials in the matrices. No copper ion release was observed on either extruded-injected PP composites or PLA composites. Although copper ion release was observed from the thin PLA composite films that were prepared extrusion-film formation processes, the amount of copper leaching from hydrophobic PLA film is insufficient to inactivate E.coli DH5 $\alpha$.

In chapter 6 is presented the effect of different drying methods on the morphology and surface chemistry of the hybrid materials. Three methods were examined to dry hybrid cellulose-copper nanoparticles suspensions that included CMC-copper nanoparticles and TEMPO nanofibrillated cellulose-copper nanoparticles (TNFC-copper nanoparticles). These methods were: (1) spray-drying, (2) freeze-drying, and (3) modified freeze drying. After the freeze-drying process, plate-like structures of cellulose and cellulose-copper nanoparticles were formed, with nano-scaled thickness but length and width over hundreds of microns. Most of the spray dried particles exhibited spherical shapes with the particle size ranging from around $300 \mathrm{~nm}$ to several microns. The spray drying process caused the most copper loss and freeze drying process caused the least copper loss. XPS results showed that the oxidation state of copper on the final dried material depends on the specific substrate. CMC surfaces exhibit mostly $\mathrm{Cu}^{0}$ and/or $\mathrm{Cu}^{+}$. On the contrary TNFC surfaces present mostly $\mathrm{Cu}^{2+}$. Solvent exchange process using ethanol and butanol creates more porous structures on the CMC substrates. A solvent exchange process using ethanol and tert-butanol sequence facilitates the formation of a more porous structure on TNFC. 
Finally, in Chapter $\mathbf{7}$ is presented the general conclusions of the current work and future work is proposed. Copper nanoparticles were successfully synthesized on two cellulosic templates [TEMPO nanofibrillated cellulose (TNFC) and carboxymethyl cellulose (CMC)]. The hybrid materials were used as a bi-functional (antimicrobial and reinforcing) filler and were incorporated into polyvinyl alcohol (PVA) matrix in wet condition. The resulting PVA composite films that were prepared using wet process exhibited strong antimicrobial activities against E.coli DH5 $\alpha$, and had enhanced thermal and mechanical performances as well. However, the incorporation of freeze-dried hybrid materials into the hydrophobic thermoplastic resins such as PLA in dry condition was detrimental to the thermal and mechanical performance. Although low amount of copper ions leaching from the films were detected, the amount of ions leaching were not enough to inactivate E.coli. The degree of agglomeration and surface chemistry of the hybrid materials not only depended on the cellulosic template, but also on the drying methods. We recommended the future work should focus on the studies on the effect of cellulosic template on the controlled release of copper ions from the thermoplastic films, the investigation of antimicrobial mechanism of copper nanoparticles on the films, and the investigations of the effects of other factors such as $\mathrm{pH}$, reducing agents, carboxylic group concentration in the cellulosic templates on the particle morphology, particles size, crystalline structure and oxidation state of copper. 
The following publications are, so far, the result of the research performed:

1) Zhong, T., Oporto, G. S., Jaczynski, J., Tesfai, A. T., \& Armstrong, J. (2013). Antimicrobial properties of the hybrid copper nanoparticles-carboxymethyl cellulose. Wood and Fiber Science, 45(2), 215-222.

2) Oporto, G. S., Zhong, T., Jaczynski, J., \& Sabo, R. (2014, June). Microstructure, mechanical, thermal and antimicrobial properties of hybrid copper nanoparticles and cellulose based materials embedded in thermoplastic resins. In 57th SWST International Convention 7th Wood Structure and Properties Conference 6th European Hardwood (p. 441).

3) Zhong, T., Oporto, G. S., Jaczynski, J., \& Jiang, C. (2015). Nanofibrillated cellulose and copper nanoparticles embedded in polyvinyl alcohol films for antimicrobial applications. BioMed Research International, 2015, 1-8.

4) Zhong, T., Oporto, G. S., Peng, Y., Xie, X., \& Gardner, D. J. (2015) Drying cellulosebased materials containing copper nanoparticles. Cellulose, 22(4), 2665-2681. 


\section{References}

Han, J. H. (2003). Antimicrobial food packaging. In: Ahvenainen R (ed) Novel food packaging techniques. CRC Press, Boca Raton, pp 50-65.

Mead, P. S., Slutsker, L., Dietz, V., McCaig, L. F., Bresee, J. S., Shapiro, C., ... \& Tauxe, R. V. (1999). Food-related illness and death in the United States. Emerging Infectious Diseases, 5(5), 607.

Suppakul, P., Miltz, J., Sonneveld, K., \& Bigger, S. W. (2003). Active packaging technologies with an emphasis on antimicrobial packaging and its applications. Journal of Food Science, 68(2), 408-420. 


\section{CHAPTER 1: LITERATURE REVIEW}

\subsection{Antimicrobial food packaging}

The growth of microorganisms on the foods not only worsens the food quality, shortens the shelf life of the food products, the contamination of food products by microorganisms or microbial toxins also can lead to serious foodborne illness. It is reported by US Centers for Disease Control and Prevention that there are approximate 76 million illnesses, 325,000 hospitalizations, and 5,000 deaths caused by foodborne disease (Mead et al. 1999). Foodborne pathogens are still the main causes for illness and death and cost billions of dollars in medical care associated with foodborne illness. Therefore the microbial growth on the packaged food would pose threats to food safety and public health. The emerging of novel antimicrobial packaging would provide a new path toward eliminating foodborne pathogens and protecting human from foodborne illness.

Conventional food packaging used to be passive packaging in the past and simply acted as container protecting food product from moisture, oxygen and physical damage. In recent years passive packaging has been developed to active packaging that is novel concept in the food packaging area. Active packaging can interact with microorganisms on the surfaces of the foods so as to reduce the number of viable microorganism, thereby increasing the shelf life and protecting the quality of products (Risch 2009). Antimicrobial packaging is one of the most promising active packaging and was prepared by incorporation of antimicrobial agents into conventional packaging to produce novel antimicrobial system. Antimicrobial packaging has a capability of inactivating or inhibiting the growth of pathogens by extending the lag period, reducing the growth rate and decreasing the number of microorganism survivors, and ultimately extending the shelf life and enhancing food safety (Han 2000).

A wide range of antimicrobial agents could be incorporated into thermoplastic resins intended for packaging to produce new novel antimicrobial packaging. The chemical 
substances, such as organic acid, fungicides, alcohols and antibiotics, are the most common antimicrobial agents. Most of organic acids have been used as food preservatives and food contact material sanitizers, which includes benzoic acids, sorbates, sorbic acid, acetic acid and lactic acid (Han 2003). Natural antimicrobial substances have attracted interests because these antimicrobial materials can be extracted from natural plants, such as grapefruit extract, clove extract, cinnamon and horseradish, these natural substances have been added to food packaging to prove effective antimicrobial activity against bacteria (Han 2003; Han 2005). The antimicrobial, antifungal, antiviral and insecticidal properties of some essential oils also have long been recognized and even have been designated as generally regarded as safe (Espitia et al. 2011). Therefore natural antimicrobial substances are desirable for the development of antimicrobial food packaging systems due to their easier regulation process when compared to the chemical processes. Some bacteriocin produced by microorganisms have also been demonstrated inhibiting the growth of spoilage and pathogenic microorganisms. These bacteriocin includes nisin, lacticin, pediocin and diplococci (Han 2003; Han 2005).

Melting-extrusion and solvent casting are two most common methods for packaging film formation, the production of packaging films often takes place in the harsh conditions such as high pressures or temperatures. During plastic fabrication process, the chemical stabilities of heat-sensitive or volatile antimicrobial agents might be affected, leading to the antimicrobial activity loss in the final products. Metallic antimicrobials have high thermal stability and low volatility, thereby metallic antimicrobial agent has advantages over some poor heat-resistant antimicrobial agents (Han 2000; Appendini and Hotchkiss 2002; De Azeredo et al. 2009).

Heavy metals, such as gold, silver, copper and zinc, have been demonstrated to possess effective antimicrobial activities against a wide variety of bacteria, they do not have adverse effects on eukaryotic cells below certain concentrations and may be good candidates for the use as antimicrobial agents (Llorens et al. 2012). Because of high specific surface areas and enhanced surface reactivity in addition to the advantages such as 
high temperature stability and low volatility, antimicrobials materials metallic nanoparticles have attracted a growing attention (Azeredo et al 2013). The antimicrobial packaging that was embedded with nanostructured antimicrobial agents such as silver nanoparticles or copper nanoparticles are able to inactivate more microorganisms when compared with higher scale counterparts (Azam et al. 2012). Bigger size and higher concentration of antimicrobial agents might affect their mechanical properties of their filled composites, whereas nanostructured antimicrobial agents can be positioned at the amorphous region of the polymeric structure without interfering the polymer and polymer interaction, the nanostructured antimicrobial agents would not damage physical and mechanical integrity (Han 2003).

According to the reviews performed by (Appendini and Hotchkiss 2002; Han 2003), antimicrobial agents could be incorporated into packaging materials through methodologies like 1) simply blending of antimicrobial agents and food packaging materials, 2) coating antimicrobial agents on the food packaging materials surface, and 3) immobilization of antimicrobials to food packaging materials via covalent binding. Four common antimicrobial packaging systems and the release profiles of antimicrobial agents were presented in Figure 1.1. The antimicrobial agents via simple blending or coating onto packaging materials could release from the packaging materials into the foods as shown in the Figure 1.1a, 1.1b, 1.1c (Han 2000; Quintavalla and Vicini 2002; Bastarrachea et al. 2011). When the regulations do not allow the migration of antimicrobial agent into the foods, antimicrobial agents could be chemically immobilized into packaging materials via covalent binding of antimicrobial substances into the structures of packaging materials as shown in Figure 1.1d (Han 2005; Bastarrachea et al. 2011). 


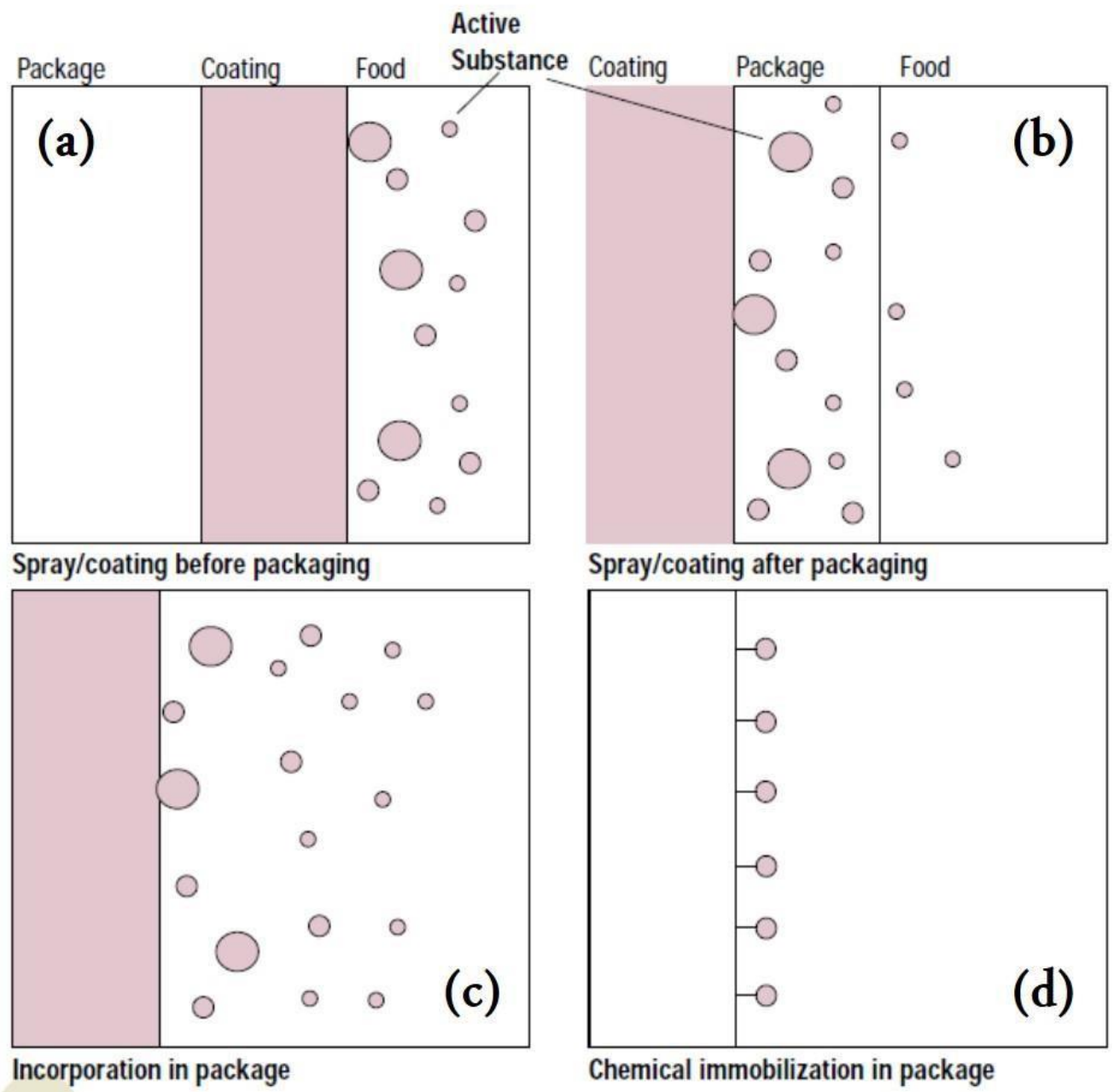

Figure 1. 1 Antimicrobial packaging systems (Han 2000; Quintavalla and Vicini 2002; Bastarrachea et al. 2011).

\subsection{Antimicrobial copper}

The antimicrobial properties of copper have long been recognized and benefited human civilization for centuries, because copper is a broad-spectrum biocide, showing effective antimicrobial activities against bacteria, fungus and viruses (Cady et al. 2011). In the ancient Egypt (2000 BC), copper was used to disinfect water and treat the wounds. In the ancient Greece (400BC), Hippocrates prescribed copper for pulmonary diseases and for purifying drinking water. The early Phoenicians nailed the copper strips to ships' hull to prevent fouling (Borkow and Gabbay 2009). 
The potent antimicrobial properties of copper, in various forms such as zero-valent copper, ionic copper, copper oxide and copper-containing molecular complexes, have been well established and recognized. Bacteria killed by copper and copper compounds include E.coli (Yoon et al. 2007; Ruparelia et al. 2008; Palza et al. 2010; Raffi et al. 2010; Ramyadevi et al. 2012; Chatterjee et al. 2014; Hassan et al. 2014; Meghana et al. 2015), Staphylococcus aureus (Ruparelia et al. 2008; Ren et al. 2009; Ramyadevi et al. 2012; Hassan et al. 2014), Campylobacter jejuni (Faundze et al. 2004), Bacillus (Yoon et al. 2007; Ruparelia et al. 2008; Pang et al. 2009), Salmonella (Faundez et al. 2003; Keyhani et al. 2006), Listeria monocytogenes (Wilks et al. 2006), Pseudomonas fluorescens (Longano et al. 2012), Shigella flexnerii (Cortes et al. 2006). Based on the large amount of antimicrobial efficacy testing conducted by the Copper Development Association (CDA), U.S. Environmental Protection Agency (EPA) allowed the CDA to make public claims that the surfaces of antimicrobial copper alloys can inactivate greater than $99.9 \%$ bacterial within two hours, and continue to eliminate more than $99 \%$ of bacteria even after repeated contamination (Borkow and Gabbay 2009). The public health claims also acknowledged that copper, brass and bronze are capable of inactivating harmful, potentially deadly bacteria, such as methicillin-resistant S. Aureus (MRSA). MRSA has been called superbug because it is resistant to many different antibiotics and is one of most virulent strains of bacteria in the hospitals (Borkow and Gabbay 2009). Copper became the first solid surface materials to receive the registration as antimicrobial material, which was approved by EPA (Llorens et al. 2012a). In contrast to microorganisms, which is extremely susceptible to copper, human tissue has low sensitivity to copper (Perelshtein et al. 2009).

Among various forms of copper species, copper nanoparticles can act as high-performance antimicrobial agents due to their high surface areas and crystal morphologies with numerous edge/corner and other reactive surface sites, which allow them to interact with cell membranes more closely (Stoimenov et al. 2002; Raffi et al. 2010; Diaz-Visurraga et al. 2012; Giannousi et al. 2014). Chatterjee et al. (2014) reported that copper nanoparticles have higher antimicrobial activity than the equivalent amount of its precursor $\mathrm{CuCl}_{2}$ or 
copper salt. Micrometric metal copper did not cause cell damage efficiently as compared with highly biocidal copper nanoparticles at the same mass (Karlsson et al. 2013).

The procuration of copper particles on the nanoscale is one of the most important steps for designing a nano-structured copper materials, because the size, shape, size distribution and stability of the nanoparticles would affect their functionalities and properties (Lagaron 2011). Copper nanoparticles would aggregate quickly in the solution as a result of van der Waals forces and larger surface area (Longano et al. 2012). The high tendency of aggregation of copper nanoparticles remains an obstacle for the development of copperbased nanocomposite products. According to the review performed by Mallick et al. (2012) and Kanninen et al. (2008), several methods have been proposed for the synthesis of copper nanoparticles that include chemical reduction, sono-chemical reduction, thermal reduction, radiation methods, metal vapor synthesis, vacuum vapor deposition, laser ablation and microemulsion techniques. One of the most preventive methods to synthesize colloidal metal particles is chemical reduction of cationic ions in the presence of organic templates, because the experiment was performed under relatively convenient operation with simple equipment. In addition, organic templates can help protect metallic nanoparticles from aggregation and reduce the extent of aggregation by lowing the surface energy by absorption of stabilizer such as polymers (Lagaron 2011). Biopolymers such as cellulose and its derivatives are promising candidates as copper stabilizer because they are readily available, inexpensive, environmentally friendly and fully compatible with scaling up industrious needs (Abdel-Halim and Al-Deyab 2011, Longano et al. 2012). Cellulose is a relatively stable polymer due to the intra- and inter-chain hydrogen bonding network, in addition, its unique nano-porous structure also make cellulose ideal for stabilizing metallic nanostructures (He et al. 2003; Dong and Hinestroza 2009; Moon et al. 2011). One of template roles of the host macromolecular chains is to help improve the distribution of copper nanoparticles inside the cellulose matrix and prevent the formation of aggregates. At the same time another important template role of the polymer chains is to narrow size distribution and well defined shape for the metallic nanoparticles (Dallas et al 2011). In this regard, cellulose was widely used as a template and stabilizer for the synthesis of 
copper nanoparticles (Kotelnikova et al. 2007; Mary et al. 2009; Perelshtein et al. 2009; Padalkar et al. 2010; Cady et al. 2011; Cao et al. 2011; Jia et al. 2012; Llorens et al. 2012b). Cellulose is ideal candidate as a template and stabilizer for copper nanoparticles and prevents the aggregation.

\subsection{Cellulose}

\subsubsection{Cellulose structure}

Cellulose is one of the most important natural polymers, it is abundant and almost inexhaustible in nature, it is not only found in plant cell walls, but it is also produced by some animal (e.g. tunicates), algae and few bacteria (Klemm et al. 2011; Kalia et al. 2014). Cellulose is a homopolymer that is composed of $\beta$-D-glucopyranose units, the repeated units are linked together by $(1 \rightarrow 4)$-glycosidic bonds. The length of a native cellulose molecules is 5000-7500 $\mathrm{nm}$ corresponding to a chain with about 10000-15000 glucopyranose units depending on the cellulose source materials (Gardner et al. 2008; Moon et al. 2011). Elementary fibril is formed via multiple cellulose chains parallel stack, and then further aggregate of elementary fibril forms larger microfibrils through van der Waals and inter- and intramolecular hydrogen bonds between hydroxyl groups and oxygens of adjacent molecules (Moon et al. 2011). The microfibrils comprise two phases crystalline regions and amorphous regions. In crystalline regions, cellulose chains are arranged in a highly ordered, whereas in amorphous regions the arrangement of cellulose chains are disordered (Gardner et al. 2008; Moon et al. 2011). High-pressure homogenization is one of most popular mechanical approaches to disintegrate the nature cellulose microfibril assembly, further multiple treatments at high pressures in combination with surface modifications such as TEMPO-mediation oxidation, microfibrillated cellulose (MFC) can be further disintegrated into nanofirillated cellulose (NFC) that was also referred to as cellulose nanofibers (CNF) and nanofibril (Wang and Drzal 2012). 


\subsubsection{Carboxylate cellulose as template for copper nanoparticles}

The general processes in which copper can be attached to cellulosic material are: 1) physical impregnation of copper into cellulose. Perelshtein et al. (2009) for instance, used ultrasound irradiation to incorporate copper oxide nanoparticles at the surface of cellulose; 2) ion-dipole interactions. Copper ions were tightly anchored on ether and hydroxyl sites via ion-dipole interactions, followed the reduction of copper ions by chemical reducing agents or UV irradiation (He et al. 2003); 3) electrostatic assembly. Cady et al. (2011), for example, grafted negative charged functional groups, such as carboxylate, on cellulose. After that, they used cationic copper ions that were electrostatically attracted to the negatively charged surface of cellulose. Negatively charged carboxylate groups can chelate cationic metal ions, thus copper ions are able to form cellulose complexes with sulfur-, nitrogen- or oxygen-containing functional groups present on the cellulose (Cady et al. 2011; Palza 2015).

There are a great deal of hydroxyl groups on the cellulose surface, C6 is a primary hydroxyl, which is the most reactive position for esterification reactions (Gardner et al. 2008). In this study carboxymethyl cellulose (CMC) and 2,2,6,6-tetramethylpiperidine-1oxyl radical (TEMPO) nanofibrillated cellulose (TNFC) were chosen as templates for the synthesis of copper nanoparticles. Both polymers were chemically modified by grafting carboxylate groups in their backbone structures. It is well known that carboxylate groups can be used as chelating groups to quantitatively capture metal ions (Ifuku et al. 2009; Saito et al. 2007, Zhong et al. 2013).

Carboxymethyl cellulose (CMC) is a cellulose derivative and obtained by replacing the C6 hydroxyl groups with carboxymethyl groups through chloroacetic acid treatment (He et al. 2007; Cady et al. 2011). Carboxymethyl cellulose is a commercial available microcrystalline cellulose which is composed of aggregate bundles of cellulose microfibrils and has the particles size ranging from 10-50 $\mu \mathrm{m}$ in diameter (Moon et al. 2011). The 
commercial carboxymethyl cellulose has a degree of polymerization ranging from 400 to 3200.

Nanofibrillated cellulose (NFC) comprises long and flexible cellulose fibrils with a high aspect ratio (4-20 nm wide, 500-2000 nm in length), and contains both crystalline and amorphous regions (Moon et al. 2011). An intense high mechanical shearing treatment is a very common approach to delaminate cellulosic fibers to produce nanofibrillated cellulose. In order to overcome inter-fibrillar hydrogen bonds in both crystalline and amorphous domains and better release the NFC, further surface oxidation of cellulose fibers was performed in combination with mechanical shearing treatment (Kalia et al. 2014). This promising and easier way to facilitate the release of NFC with low energy mechanical disintegration is to use 2,2,6,6-tetramethylpiperidine-1-oxyl (TEMPO)-mediated oxidation that was first reported by Saito et al. (2004). TEMPO is a water-soluble and highly stable nitroxyl radicals, which can efficiently and selectively oxidize alcoholic hydroxyls to aldehydes, ketones and carboxyl groups under mild conditions (Isogai et al. 2011; Kalia et al. 2014). After TEMPO-mediated oxidation, negatively charged carboxylate groups were introduced into cellulosic materials through regioselective conversion of C6 primary hydroxyls to carboxylate groups (Saito and Isogai 2004; Isogai et al. 2011). The negative charge on cellulose surface leads to the repulsion of the nanofibers, thus easing fibrillation (Siro and Plackett 2010). Depending on TEMPO oxidation conditions such as $\mathrm{pH}$ and the amount of $\mathrm{NaClO}$, TNFC has a degree of polymerization ranging from 200 to 600 (Isogai et al. 2011; Moon et al. 2011; Saito and Isogai 2004).

\subsection{References}

Abdel-Halim, E. S., \& Al-Deyab, S. S. (2011). Utilization of hydroxypropyl cellulose for green and efficient synthesis of silver nanoparticles. Carbohydrate Polymers, 86(4), $1615-1622$.

Appendini, P., \& Hotchkiss, J. H. (2002). Review of antimicrobial food packaging. Innovative Food Science \& Emerging Technologies, 3(2), 113-126. 
Azam, A., Ahmed, A. S., Oves, M., Khan, M. S., \& Memic, A. (2012). Size-dependent antimicrobial properties of $\mathrm{CuO}$ nanoparticles against Gram-positive and-negative bacterial strains. International Journal of Nanomedicine, 7, 3527.

Bastarrachea, L., Dhawan, S., \& Sablani, S. S. (2011). Engineering properties of polymeric-based antimicrobial films for food packaging: a review. Food Engineering Reviews, 3(2), 79-93.

Borkow, G., \& Gabbay, J. (2009). Copper, an ancient remedy returning to fight microbial, fungal and viral infections. Current Chemical Biology, 3(3), 272-278.

Cady, N. C., Behnke, J. L., \& Strickland, A. D. (2011). Copper-Based Nanostructured Coatings on Natural Cellulose: Nanocomposites Exhibiting Rapid and Efficient

Inhibition of a Multi-Drug Resistant Wound Pathogen, A. baumannii, and Mammalian Cell Biocompatibility In Vitro. Advanced Functional Materials, 21(13), 2506-2514.

Cao, J., Xu, R., Tang, H., Tang, S., \& Cao, M. (2011). Synthesis of monodispersed CMCstabilized $\mathrm{Fe}-\mathrm{Cu}$ bimetal nanoparticles for in situ reductive dechlorination of 1, 2, 4trichlorobenzene. Science of the Total Environment, 409(11), 2336-2341.

Chatterjee, A. K., Chakraborty, R., \& Basu, T. (2014). Mechanism of antibacterial activity of copper nanoparticles. Nanotechnology, 25(13), 135101.

Cortés, P., Atria, A. M., Contreras, M., Garland, M. T., Peña, O., \& Corsini, G. (2006). Magnetic properties and antibacterial activity of tetranuclear copper complexes bridged by oxo group. Journal of the Chilean Chemical Society, 51(3), 957-960. 
Dallas, P., Sharma, V. K., \& Zboril, R. (2011). Silver polymeric nanocomposites as advanced antimicrobial agents: classification, synthetic paths, applications, and perspectives. Advances in Colloid and Interface Science, 166(1), 119-135.

De Azeredo, H. M. (2009). Nanocomposites for food packaging applications. Food Research International, 42(9), 1240-1253.

De Azeredo, H. M. (2013). Antimicrobial nanostructures in food packaging. Trends in Food Science \& Technology, 30(1), 56-69.

Díaz-Visurraga, J., Gutiérrez, C., Von Plessing, C., \& García, A. (2011). Metal nanostructures as antibacterial agents. Science and Technology against Microbial Pathogens: Research, Development and Evaluation. Badajoz: Formatex, 210-218.

Dong, B. H., \& Hinestroza, J. P. (2009). Metal nanoparticles on natural cellulose fibers: electrostatic assembly and in situ synthesis. ACS Applied Materials \& Interfaces, 1(4), 797-803.

Espitia, P. J., Soares, N. D. F., Botti, L., \& Silva, W. A. (2011, January). Effect of essential oils in the properties of cellulosic active packaging. In Macromolecular Symposia (Vol.

299, No. 1, pp. 199-205). WILEY-VCH Verlag.

Faúndez, G., Troncoso, M., Navarrete, P., \& Figueroa, G. (2004). Antimicrobial activity of copper surfaces against suspensions of Salmonella enterica and Campylobacter jejuni. BMC Microbiology, 4(1), 19.

Giannousi, K., Lafazanis, K., Arvanitidis, J., Pantazaki, A., \& Dendrinou-Samara, C. (2014). Hydrothermal synthesis of copper based nanoparticles: antimicrobial screening and interaction with DNA. Journal of Inorganic Biochemistry, 133, 24-32. 
Gardner, D. J., Oporto, G. S., Mills, R., \& Samir, M. A. S. A. (2008). Adhesion and surface issues in cellulose and nanocellulose. Journal of Adhesion Science and Technology, 22(5-6), 545-567.

Gabbay, J., Borkow, G., Mishal, J., Magen, E., Zatcoff, R., \& Shemer-Avni, Y. (2006). Copper oxide impregnated textiles with potent biocidal activities. Journal of Industrial Textiles, 35(4), 323-335.

Han, J. H. (2000). Antimicrobial food packaging. Food Technology, 54(3), 56-65.

Han, J. H. (2003). Antimicrobial food packaging. In: Ahvenainen R (ed) Novel food packaging techniques. CRC Press, Boca Raton, pp 50-65.

Han, J. H. (2005). Antimicrobial food packaging. In: Han JH (ed) Innovations in food packaging. Elsevier Academic Press, San Diego, pp 81-107.

Hassan, I. A., Parkin, I. P., Nair, S. P., \& Carmalt, C. J. (2014). Antimicrobial activity of copper and copper (I) oxide thin films deposited via aerosol-assisted CVD. Journal of Materials Chemistry B, 2(19), 2855-2860.

He, F., Zhao, D., Liu, J., \& Roberts, C. B. (2007). Stabilization of Fe-Pd nanoparticles with sodium carboxymethyl cellulose for enhanced transport and dechlorination of trichloroethylene in soil and groundwater. Industrial \& Engineering Chemistry Research, 46(1), 29-34.

He, J., Kunitake, T., \& Nakao, A. (2003). Facile in situ synthesis of noble metal nanoparticles in porous cellulose fibers. Chemistry of Materials, 15(23), 4401-4406. 
Ifuku, S., Tsuji, M., Morimoto, M., Saimoto, H., \& Yano, H. (2009). Synthesis of silver nanoparticles templated by TEMPO-mediated oxidized bacterial cellulose nanofibers. Biomacromolecules, 10(9), 2714-2717.

Isogai, A., Saito, T., \& Fukuzumi, H. (2011). TEMPO-oxidized cellulose nanofibers. Nanoscale, 3(1), 71-85.

Jia, B., Mei, Y., Cheng, L., Zhou, J., \& Zhang, L. (2012). Preparation of copper nanoparticles coated cellulose films with antibacterial properties through one-step reduction. ACS Applied Materials \& Interfaces, 4(6), 2897-2902.

Kalia, S., Boufi, S., Celli, A., \& Kango, S. (2014). Nanofibrillated cellulose: surface modification and potential applications. Colloid and Polymer Science, 292(1), 5-31.

Kanninen, P., Johans, C., Merta, J., \& Kontturi, K. (2008). Influence of ligand structure on the stability and oxidation of copper nanoparticles. Journal of Colloid and Interface Science, 318(1), 88-95.

Karlsson, H. L., Cronholm, P., Gustafsson, J., \& Moller, L. (2008). Copper oxide nanoparticles are highly toxic: a comparison between metal oxide nanoparticles and carbon nanotubes. Chemical Research in Toxicology, 21(9), 1726-1732.

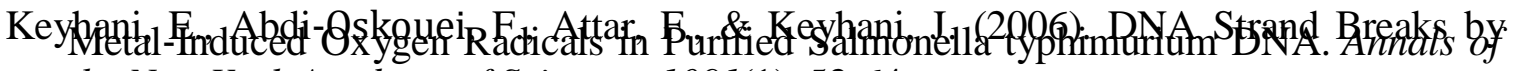
the New York Academy of Sciences, 1091(1), 52-64.

Klemm, D., Kramer, F., Moritz, S., Lindström, T., Ankerfors, M., Gray, D., \& Dorris, A. (2011). Nanocelluloses: a new family of nature-based materials. Angewandte Chemie International Edition, 50(24), 5438-5466. 
Kotelnikova, N., Vainio, U., Pirkkalainen, K., \& Serimaa, R. (2007, August). Novel Approaches to Metallization of Cellulose by Reduction of Cellulose-Incorporated Copper and Nickel Ions. In Macromolecular Symposia (Vol. 254, No. 1, pp. 74-79).

\section{WILEY-VCH Verlag.}

Lagaron, J. M. (Ed.). (2011). Multifunctional and nanoreinforced polymers for food packaging. Elsevier.

Llorens, A., Lloret, E., Picouet, P. A., Trbojevich, R., \& Fernandez, A. (2012). Metallicbased micro and nanocomposites in food contact materials and active food packaging. Trends in Food Science \& Technology, 24(1), 19-29.

Llorens, A., Lloret, E., Picouet, P., \& Fernandez, A. (2012b). Study of the antifungal potential of novel cellulose/copper composites as absorbent materials for fruit juices. International Journal of Food Microbiology, 158(2), 113-119.

Longano, D., Ditaranto, N., Sabbatini, L., Torsi, L., \& Cioffi, N. (2012). Synthesis and antimicrobial activity of copper nanomaterials. In Nano-Antimicrobials (pp. 85-117). Springer Berlin Heidelberg.

Longano, D., Ditaranto, N., Cioffi, N., Di Niso, F., Sibillano, T., Ancona, A., ... \& Torsi, L. (2012). Analytical characterization of laser-generated copper nanoparticles for antibacterial composite food packaging. Analytical and Bioanalytical Chemistry, 403(4), 1179-1186.

Mallick, S., Sharma, S., Banerjee, M., Ghosh, S. S., Chattopadhyay, A., \& Paul, A. (2012). Iodine-stabilized $\mathrm{Cu}$ nanoparticle chitosan composite for antibacterial applications. ACS Applied Materials \& Interfaces, 4(3), 1313-1323. 
Mary, G., Bajpai, S. K., \& Chand, N. (2009). Copper (II) ions and copper nanoparticles-loaded chemically modified cotton cellulose fibers with fair antibacterial properties. Journal of Applied Polymer Science, 113(2), 757-766.

Mead, P. S., Slutsker, L., Dietz, V., McCaig, L. F., Bresee, J. S., Shapiro, C., Griffin, p. M., \& Tauxe, R. V. (1999). Food-related illness and death in the United States. Emerging Infectious Diseases, 5(5), 607.

Meghana, S., Kabra, P., Chakraborty, S., \& Padmavathy, N. (2015). Understanding the pathway of antibacterial activity of copper oxide nanoparticles. RSC Advances, 5, 12293-12299.

Moon, R. J., Martini, A., Nairn, J., Simonsen, J., \& Youngblood, J. (2011). Cellulose nanomaterials review: structure, properties and nanocomposites. Chemical Society Reviews, 40(7), 3941-3994.

Padalkar, S., Capadona, J. R., Rowan, S. J., Weder, C., Won, Y. H., Stanciu, L. A., \& Moon, R. J. (2010). Natural biopolymers: novel templates for the synthesis of nanostructures. Langmuir, 26(11), 8497-8502.

Palza, H., Gutiérrez, S., Delgado, K., Salazar, O., Fuenzalida, V., Avila, J. I., Figueroa, G., \& Quijada, R. (2010). Toward Tailor-Made Biocide Materials Based on Poly (propylene)/Copper Nanoparticles. Macromolecular Rapid Communications, 31(6), 563-567.

Palza, H. (2015). Antimicrobial polymers with metal nanoparticles. International Journal of Molecular Sciences, 16, 2099-2116.

Pang, H., Gao, F., \& Lu, Q. (2009). Morphology effect on antibacterial activity of cuprous oxide. Chemical Communications, (9), 1076-1078. 
Perelshtein, I., Applerot, G., Perkas, N., Wehrschuetz-Sigl, E., Hasmann, A., Guebitz, G., \& Gedanken, A. (2009). CuO-cotton nanocomposite: formation, morphology, and antibacterial activity. Surface and Coatings Technology, 204(1), 54-57.

Quintavalla, S., \& Vicini, L. (2002). Antimicrobial food packaging in meat industry. Meat Science, 62(3), 373-380.

Raffi, M., Mehrwan, S., Bhatti, T. M., Akhter, J. I., Hameed, A., Yawar, W., \& ul Hasan, M. M. (2010). Investigations into the antibacterial behavior of copper nanoparticles against Escherichia coli. Annals of Microbiology, 60(1), 75-80.

Ramyadevi, J., Jeyasubramanian, K., Marikani, A., Rajakumar, G., \& Rahuman, A. A. (2012). Synthesis and antimicrobial activity of copper nanoparticles. Materials Letters, 71, 114-116.

Ren, G., Hu, D., Cheng, E. W., Vargas-Reus, M. A., Reip, P., \& Allaker, R. P. (2009). Characterisation of copper oxide nanoparticles for antimicrobial applications. International Journal of Antimicrobial Agents, 33(6), 587-590.

Risch, S. J. (2009). Food packaging history and innovations. Journal of Agricultural and Food Chemistry, 57(18), 8089-8092.

Ruparelia, J. P., Chatterjee, A. K., Duttagupta, S. P., \& Mukherji, S. (2008). Strain specificity in antimicrobial activity of silver and copper nanoparticles. Acta Biomaterialia, 4(3), 707-716.

Saito, T., \& Isogai, A. (2004). TEMPO-mediated oxidation of native cellulose. The effect of oxidation conditions on chemical and crystal structures of the water-insoluble fractions. Biomacromolecules, 5(5), 1983-1989. 
Saito, T., Kimura, S., Nishiyama, Y., \& Isogai, A. (2007). Cellulose nanofibers prepared by TEMPO-mediated oxidation of native cellulose. Biomacromolecules, 8(8), 2485-2491.

Siró, I., \& Plackett, D. (2010). Microfibrillated cellulose and new nanocomposite materials: a review. Cellulose, 17(3), 459-494.

Stoimenov, P. K., Klinger, R. L., Marchin, G. L., \& Klabunde, K. J. (2002). Metal oxide nanoparticles as bactericidal agents. Langmuir, 18(17), 6679-6686.

Wang, T., \& Drzal, L. T. (2012). Cellulose-nanofiber-reinforced poly (lactic acid) composites prepared by a water-based approach. ACS Applied Materials \& Interfaces, 4(10), 5079-5085.

Wilks, S. A., Michels, H. T., \& Keevil, C. W. (2006). Survival of Listeria monocytogenes Scott A on metal surfaces: implications for cross-contamination. International Journal of Food Microbiology, 111(2), 93-98.

Yoon, K. Y., Byeon, J. H., Park, J. H., \& Hwang, J. (2007). Susceptibility constants of Escherichia coli and Bacillus subtilis to silver and copper nanoparticles. Science of the Total Environment, 373(2), 572-575. 


\section{CHAPTER 2: ANTIMICROBIAL PROPERTIES OF THE HYBRID COPPER NANOPARTICLES-CARBOXYMETHYL CELLULOSE}

\subsection{Chapter abstract}

In this study, a simple method to produce a cellulose-based material with antimicrobial properties was developed by introducing copper nanoparticles on carboxymethyl cellulose (CMC) using sodium borohydride as a copper reducing agent. The hybrid material was characterized by scanning electron microscopy (SEM), energy-dispersive Xray spectroscopy (EDX), and transmission electron microscopy (TEM). SEM and EDX analysis confirmed the formation of copper nanoparticles within the CMC matrix. TEM indicated a 10- to 20-nm diameter of copper nanoparticles. Antimicrobial properties of the hybrid material were effectively evaluated against the nonpathogenic surrogate of foodborne pathogen Escherichia coli.

\subsection{Introduction}

Nanotechnology is currently used as a transformational tool to develop and enhance highvalue products from renewable raw materials, such as wood. Cellulose, the most important skeletal component of wood, can be used not only in fields in which biocompatibility and biodegradability are relevant, but also potentially as a support structure for nanoparticles in promoting novel applications. One interesting application is the use of cellulose nanostructure as a template and stabilizer for metallic nanoparticles with a goal of use as an antimicrobial nanocomposite for packaging materials (Duncan 2011; Cushen et al. 2012; Llorens et al. 2012). Copper is widely known as a broadspectrum biocide. Various forms of copper, such as zero-valent copper, copper oxide, ionic copper, and copper-containing molecular complexes, have all shown antimicrobial properties (Cioffi et al. 2005; Gabbay et al. 2006; Ruparelia et al. 2008; Hu et al. 2009; Pang et al. 2009; Ren et al. 2009; Cady et al. 2011). 
In contrast to the low sensitivity of human tissue to copper (Hostynek and Maibach 2004), prokaryotes (i.e. bacteria) are extremely susceptible to copper. US Environmental Protection Agency approved the registration of copper alloys in February 2008 because copper has been demonstrated eliminating some bacteria linked to potentially fatal microbial infections. They also confirmed the antimicrobial efficacy of copper against Escherichia coli O157:H7, Staphylococcus aureus, Enterobacter aerogenes, and Pseudomonas aeruginosa (Llorens et al. 2012). The mechanism of metal nanoparticle action against microorganisms has not been clearly established. The traditional hypothesis was that metal ions are able to attach to the negatively charged bacterial cell wall, disrupting cell wall permeability to produce protein denaturation and finally induce cell death. Cioffi et al. (2005) and Anyaogu et al. (2008) agree with this hypothesis because they attribute the antibacterial property of copper to the $\mathrm{Cu}^{2+}$ ions released in aqueous medium. Cady et al (2011), Jia et al. (2012), and Mallick et al. (2012) indicated that the antimicrobial properties of copper nanoparticles against bacteria may not be simply caused by enhanced release of metal ions, but they may be also associated with contact killing.

According to reviews performed by Kanninen et al. (2008) and Mallick et al. (2012), several methods have been developed for the synthesis of copper nanoparticles, including thermal reduction, sono-chemical reduction, metal vapor synthesis, chemical reduction, vacuum vapor deposition, radiation methods, microemulsion techniques, and laser ablation. Most of these methods have been performed in a nitrogen atmosphere, because copper nanoparticles get readily oxidized in an aqueous environment in an open atmosphere (Kanninen et al. 2008; Mallick et al. 2012; Pinto et al. 2012). With the focus on simple routes to produce copper nanoparticles in an aqueous solution and air environment, using organic material proved to be effective in protecting copper from fast oxidation. In this regard, chitin, cotton, and cellulosic fibers and films were technically useful as a template or nanoreactor as well as a good reducing agent and an excellent stabilizer for copper nanoparticles (Kotelnikova et al. 2007; Nadagouda and Varma 2007; 
Mary et al. 2009; Perelshtein et al. 2009; Padalkar et al. 2010; Jia et al. 2012; Mallick et al. 2012; Pinto et al. 2012).

Kotelnikova et al. (2007) synthesized copper nanoparticles into insoluble microcrystalline cellulose by reducing the number of metal ions with sodium borohydride and hydrazine in the presence of sulfuric acid. The media used in the experiments included water and ammonia, and copper nanoparticles were in the range of 5-55 nm. Mary et al. (2009) synthesized copper nanoparticles (average diameter of $28 \mathrm{~nm}$ ) on a chitosan-bound cellulose composite using sodium borohydride as a reducing agent. The authors initially induced the oxidation of cellulose followed by covalent attachment of biopolymer chitosan. Perelshtein et al. (2009) synthesized copper oxide nanoparticles, with an average of $15 \mathrm{~nm}$, and deposited them on cotton fabrics using ultrasound and ammonium as a solution medium. The resulting material possessed effective bactericidal properties. Padalkar et al. (2010) used a multistep process to synthesize metallic nanoparticles (5 nm diameter average sizes for copper nanoparticles) on the surface of tunicate cellulose nanocrystals. The authors used the cationic surfactant cetyltrimethylammonium bromide as a stabilizer of metallic nanoparticles.

Cady et al. (2011) described a layer-by-layer electrostatic self-assembly process for fabricating an antimicrobial nanocoating on a chemically modified cotton substrate. These authors introduced carboxylic groups on the cotton surface for chelation-controlled binding of cupric ions, $\mathrm{Cu}$ (II), followed by chemical reduction with sodium borohydride to yield a nanostructured coating on cotton fibers. The resulting composites demonstrated efficiency in activation of a multidrug-resistant bacterial wound pathogen, Acinetobacter baumannii.

Mallick et al. (2012) synthesized copper nanoparticles between 8 and $12 \mathrm{~nm}$ on average in chitosan polymer using iodine as a stabilizing agent. Hydrazine was used as the reducing agent, and the final nanocomposite demonstrated effective antimicrobial properties against Gram-positive and -negative bacteria. Pinto et al. (2012) demonstrated 
that the cellulose fiber microstructure (vegetable and bacterial) protected copper nanostructures from further oxidation. Jia et al. (2012) improved the antibacterial properties of regenerated cellulose films against $S$. aureus and E. coli by embedding copper nanoparticles on the surface of the films. They used a cellulose-cuprammonium solution in alkaline medium and subsequent reduction using sodium borohydride. In this study, CMC was used as a novel template for copper nanoparticles with respect to their high carboxylic group availability. That fact was expected to improve the copper ion chelation phenomena. The antimicrobial properties of the hybrid CMC-copper nanoparticles were demonstrated against the nonpathogenic surrogate of foodborne pathogen E. coli.

\subsection{Material and methods}

\subsubsection{Materials}

Sodium carboxymethyl cellulose (NaCMC) (average molecular weight: 90,000) from Sigma Aldrich, USA; copper sulfate $\left(\mathrm{CuSO}_{4} \cdot 5 \mathrm{H} 2 \mathrm{O}\right.$; Technical-Crystal) from Fisher Scientific, USA; sodium borohydride $\left(\mathrm{NaBH}_{4}, 0.5 \mathrm{M}\right.$ solution in diglyme, Acroseal) from

Acros Organics, USA; nonpathogenic E. coli DH5 $\alpha$ from Invitrogen Inc.,USA ( $\alpha$ substrain of DH5 described by Hanahan in 1985, "DH" stands for Douglas Hanahan) is a very sensitive microorganism. This $E$. coli contains mutations of the recA and gyrA (gyrase subunit A) genes that are necessary for DNA repair and replication. Therefore, recA and gyrA mutants have impaired ability to repair and recombine their DNA strands making the mutants sensitive to any stress including chemicals. This is why this sensitive and non-pathogenic E. coli was selected as a model microbial target in this study. Sterile tryticase soy broth (TSB) from Becton Dickinson, USA; Petrifilm E. coli/Coliform count plate from Hardy Diagnostics, USA; Butterfield phosphate buffer from Hardy Diagnostics, USA. 


\subsubsection{Preparation of copper-carboxymethyl cellulose composite material}

One gram of $\mathrm{NaCMC}$ was dissolved in $49 \mathrm{~mL}$ distilled water under vigorous magnetic stirring. The resulting solution was approximately $50 \mathrm{~mL}$ of NaCMC solution $(20 \mathrm{~g} / \mathrm{L})$. Ten milliliters of copper sulfate $(0.1 \mathrm{~mol} / \mathrm{L})$ was added by drops into the $\mathrm{CMC}$ solution. The mixture was subjected to high-speed mixing while the copper sulfate solution was added. Then the mixture was allowed to react at room temperature for $3 \mathrm{~h}$. The excess of cupric ions was removed by placing the mixture into dialysis tubing, and the washing process was performed with distilled water for $24 \mathrm{~h}$. After that, $\mathrm{Cu}^{2+}$ ions were reduced to metallic copper by introducing $4 \mathrm{~mL}$ of reducing agent sodium borohydride $(0.5 \mathrm{M})$. The resulting copper-CMC mixture was stored in an airtight container attached to a vacuum pump to prevent further oxidation of the copper. This mixture was used for antimicrobial testing and transmission electron microscopy (TEM) analysis. Films of the copper-CMC composite were prepared by the solvent casting process in which the resulting $\mathrm{CMC}-$ copper solution on the glass was air-dried at room temperature for 1 week. Dried films were analyzed using scanning electron microscopy (SEM) and energy-dispersive X-ray spectroscopy (EDX).

\subsubsection{Scanning electron microscopy-energy-dispersive X-ray spectroscopy characterization}

The morphology and chemical composition of films were characterized using a JEOL (Tokyo, Japan) JSM 7600F SEM equipped with an INCA X-ray microanalysis systemEDX. Dried pure CMC films and copper-CMC films were previously coated with platinum using the sputtering process.

\subsubsection{Transmission electron microscopy characterization}

TEM analysis was carried out on a JEOL TEM-2100 instrument operating at $200 \mathrm{kV}$. A drop of freshly prepared copper-CMC mixture, with copper concentration of $1 \mathrm{~g} / \mathrm{L}$, was 
diluted to a copper concentration of $9.1 \mathrm{mg} / \mathrm{mL}$. The drop was deposited on a carboncoated copper grid, which was followed by air-drying overnight.

\subsubsection{Antimicrobial properties of copper-carboxymethyl cellulose solution against nonpathogenic Escherichia coli $\mathrm{DH} 5 \alpha$}

Antimicrobial efficacy of $\mathrm{Cu}-\mathrm{CMC}$ solution was evaluated against nonpathogenic $E$. coli DH5 $\alpha$. E. coli DH5a lyfo-disks were reconstituted by crushing one pellet using a sterile spatula in $0.5 \mathrm{~mL}$ of sterile TSB. The content was aseptically transferred to $4.5 \mathrm{~mL}$ of sterile TSB and allowed to grow aerobically at $37{ }^{\circ} \mathrm{C}$ for $24 \mathrm{~h}$ in an incubator/shaker set at 150 rpm (C24 Incubator/Shaker; New Brunswick Scientific, Edison, USA). The 5-mL aliquot of E. coli culture was aseptically transferred to $95 \mathrm{~mL}$ of sterile TSB and allowed to grow aerobically at $37{ }^{\circ} \mathrm{C}$ for $18 \mathrm{~h}$ in the incubator/shaker set at $150 \mathrm{rpm}$. This procedure yielded an 18-h E.coli culture at a stationary phase of growth and approximately $10^{8}$ colony-forming units (CFU)/mL (Tesfai et al. 2011). The bacterial enumeration procedure is subsequently described. The 18-h E.coli culture was used in subsequent experiments with the $\mathrm{Cu}-\mathrm{CMC}$ solution. A $10-\mathrm{mL}$ aliquot of the 18 -h $E$. coli culture was mixed with $40 \mathrm{~mL}$ of the $\mathrm{Cu}-\mathrm{CMC}$ solution. The mixture was gently mixed for $10 \mathrm{~h}$ at $4{ }^{\circ} \mathrm{C}$ followed by enumeration of E. coli survivors.

A serial 10-fold dilution procedure was performed to enumerate E. coli in the 18-h culture and following the 10-h exposure of E. coli to the $\mathrm{Cu}-\mathrm{CMC}$ solution (Black and Jaczynski 2006; Levanduski and Jaczynski 2008). A 10-mL aliquot of the 18-h E. coli culture or the E. coli/ $\mathrm{Cu}-\mathrm{CMC}$ mixture was aseptically mixed with diluent (Butterfield phosphate buffer). Further serial dilutions were aseptically made by taking $10 \mathrm{~mL}$ of diluted sample and transferring it to a $90-\mathrm{mL}$ diluent bottle followed by shaking the bottle to uniformly distribute bacterial cells. Survivors were enumerated on selective medium (Petrifilm E. coli/Coliform Count Plate) using a standard spread-plating technique. A 1.0$\mathrm{mL}$ aliquot of each serial dilution was pipetted and spread on $3 \mathrm{M}$ Petrifilm plates. The 3M Petrifilm plates were incubated at $35^{\circ} \mathrm{C}$ for $48 \mathrm{~h}$ (AOAC 1995, method 991.14). All 
bacterial enumerations were performed in duplicate, and mean values are reported as $\mathrm{CFU} / \mathrm{mL}$.

\subsection{Results and discussion}

\subsubsection{Scanning electron microscopy-energy-dispersive X-ray spectroscopy characterization of pure carboxymethyl cellulose and carboxymethyl cellulose- copper nanoparticle films}

Figure 2.1 presents the surface morphology of 1) a control sample of CMC film; and 2) a $\mathrm{Cu}-\mathrm{CMC}$ composite film. The presence of "big" and "small" spherical particles with an average size of $2.68 \pm 0.34 \mu \mathrm{m}$ and $0.27 \pm 0.09 \mu \mathrm{m}$, respectively, caused the different morphology on the surface of the films. Particle size was determined using Image $\mathrm{J}$ (Ferreira and Rasband 2012) processing program. Particles were analyzed using SEM and EDX. Results indicated that the big spherical particles corresponded mainly to carbon, oxygen, and sodium elements (Figure 2.2; Table 2.1); no copper was present on these particles. The analysis on the small particles revealed the presence of copper in addition to carbon, oxygen, and sodium (Figure 2.3; Table 2.2).
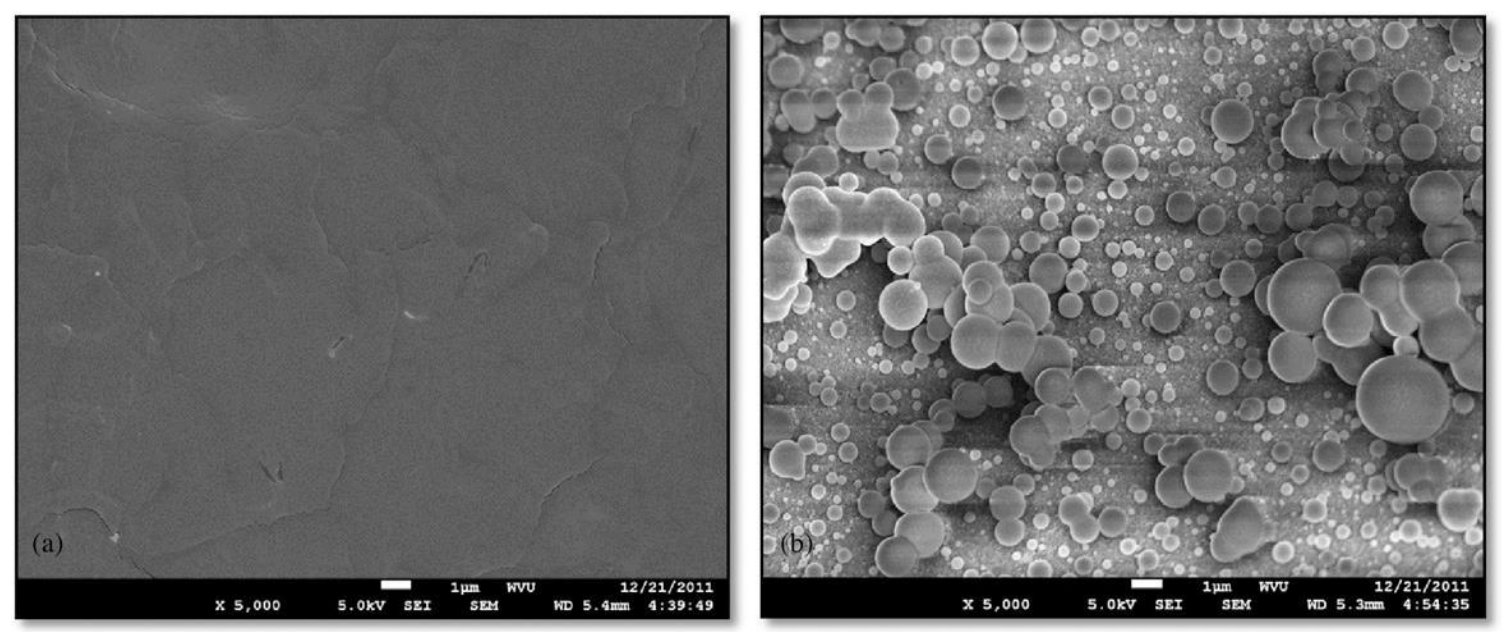

Figure 2. 1 (a) Scanning electron microscope (SEM) image of pure carboxymethyl cellulose $(\mathrm{CMC})$ film (scale bar $=1 \mu \mathrm{m})$; (b) SEM image of CMC-copper particles film (scale bar $=1 \mu \mathrm{m})$. 


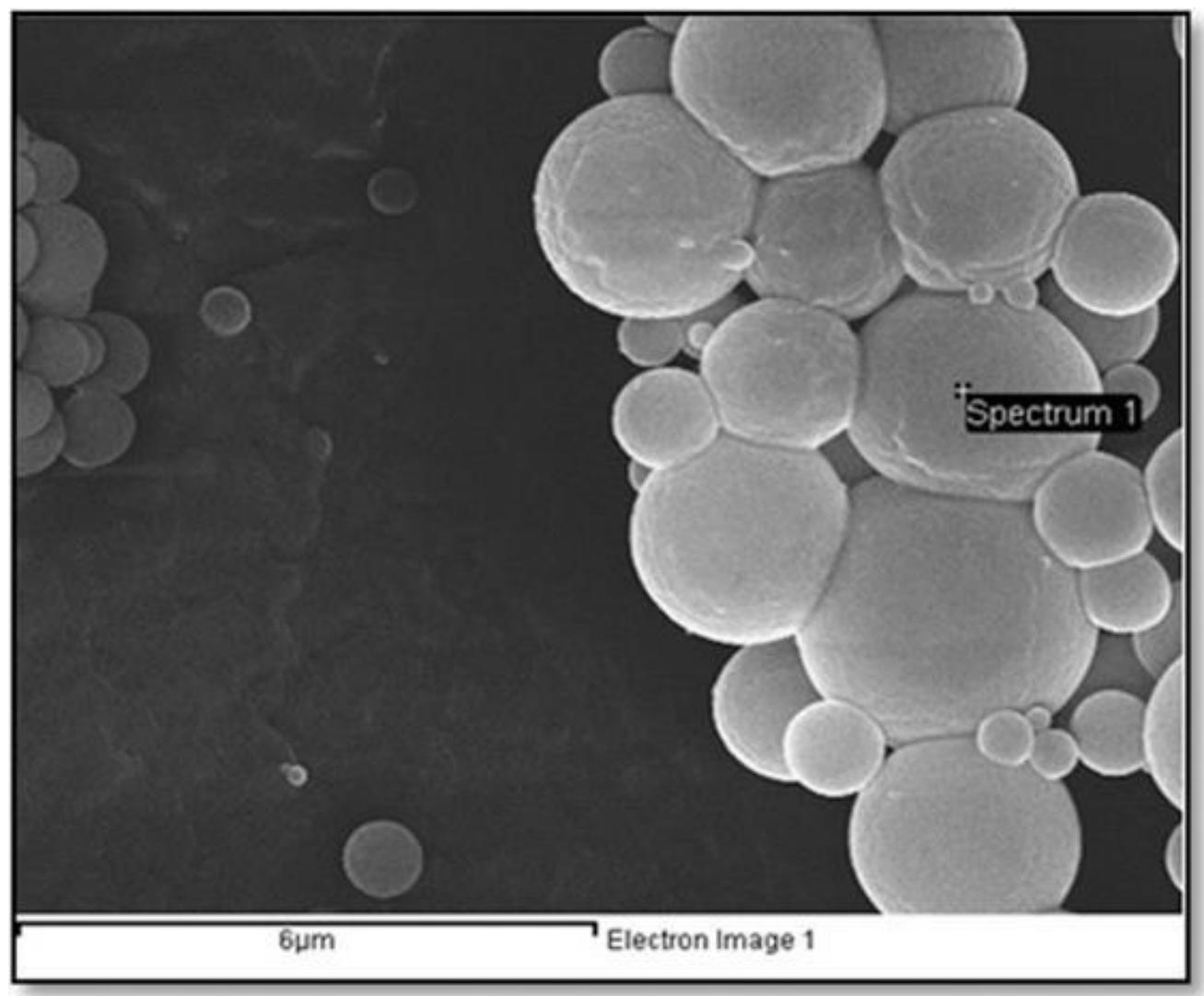

Figure 2. 2 Scanning electron microscope (SEM) image of "big" spherical particles present on the surface of the hybrid carbox ymethyl cellulose-copper film (scale bar $=6 \mu \mathrm{m}$ ). Spectrum 1 shows the place of energy-dispersive X-ray spectroscopy analysis (Table 2.1).

Table 2. 1 Energy-dispersive X-ray spectroscopy (EDX) analysis on the spherical 'big' particles present on the surface of carboxymethyl cellulose-copper nanoparticle films (Figure 2.2).

\begin{tabular}{cc}
\hline Element & Weight $^{\mathrm{a}}(\%)$ \\
\hline Carbon (C) & 39.44 \\
Oxygen (O) & 35.24 \\
Sodium (Na) & 13.9 \\
Platinum (Pt) & 11.41 \\
Total & 100 \\
\hline
\end{tabular}

${ }^{a}$ Weight percentage corresponds to the ratio of the atomic mass of the specific element and the total atomic mass. 
Table 2. 2 Energy-dispersive X-ray spectroscopy (EDX) analysis on the spherical 'small' particles present on the surface of carboxymethyl cellulose-copper nanoparticle films (Figure 2.3).

\begin{tabular}{cc}
\hline Element & Weight $^{\mathrm{a}}(\%)$ \\
\hline Carbon $(\mathrm{C})$ & 28.38 \\
Oxygen $(\mathrm{O})$ & 19.48 \\
Sodium $(\mathrm{Na})$ & 12.10 \\
Copper $(\mathrm{Cu})$ & 22.70 \\
Platinum $(\mathrm{Pt})$ & 17.35 \\
Total & 100 \\
\hline
\end{tabular}

${ }^{a}$ Weight percentage corresponds to the ratio of the atomic mass of the specific element and the total atomic mass.

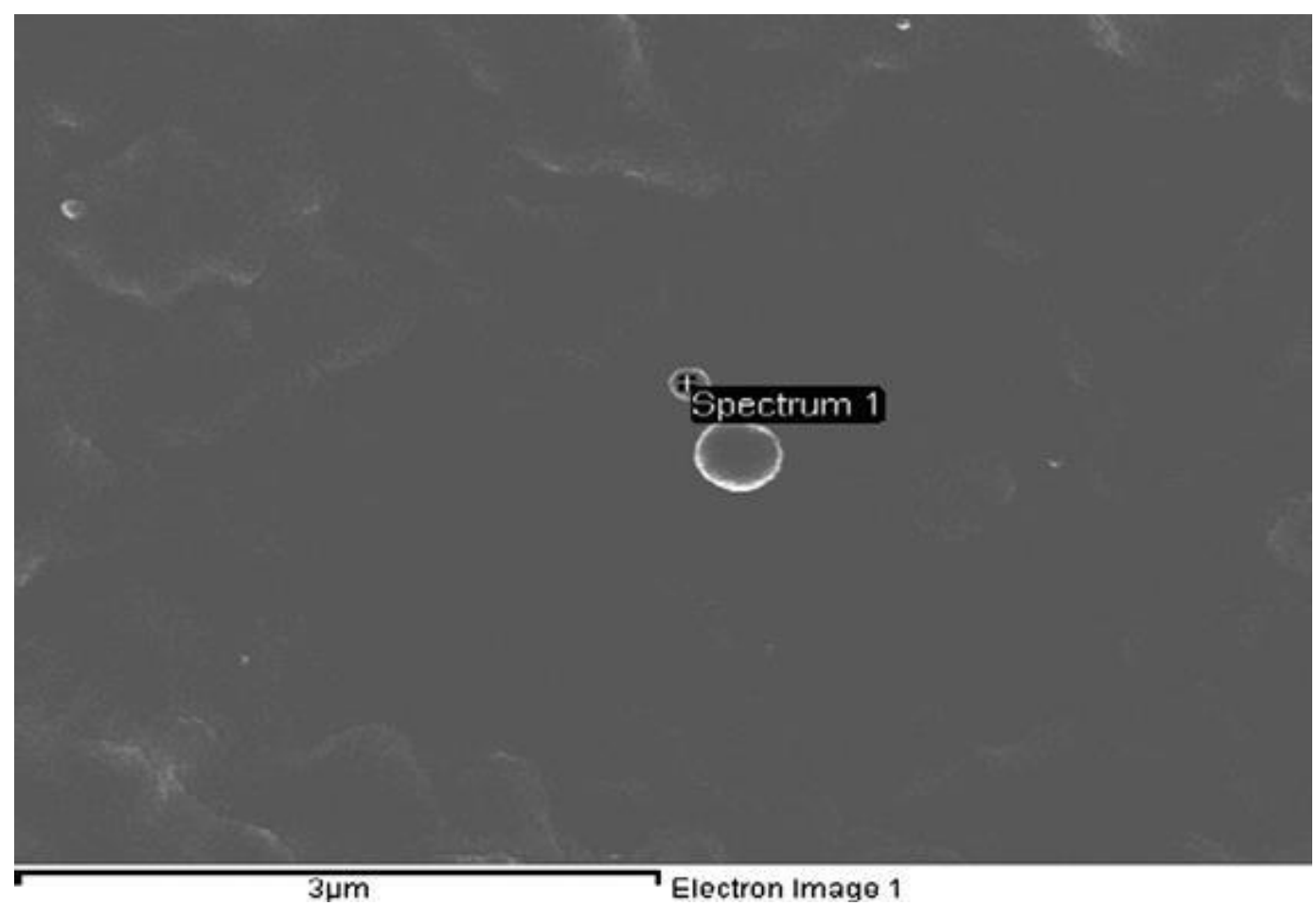

Figure 2. 3 Scanning electron microscope image of a "small" spherical particle present on the surface of the hybrid carboxymethyl cellulose-copper nanoparticles film. Spectrum 1 shows the place of energy-dispersive X-ray spectroscopy analysis (Table 2.2). 


\subsubsection{Transmission electron microscopy analysis of carboxymethyl cellulose-copper nanoparticles solution}

Figures 2.4a and 2.4b display the TEM and high-resolution TEM (HRTEM) images of a $\mathrm{Cu}-\mathrm{CMC}$ solution, respectively. Figure $2.4 \mathrm{a}$ confirms that copper nanoparticles were present on the surface of the CMC polymer. Particle sizes ranged from 10 to $20 \mathrm{~nm}$. The HRTEM image of single-crystalline copper nanoparticles on the CMC structure displays lattice fringes (Figure 2.4b).
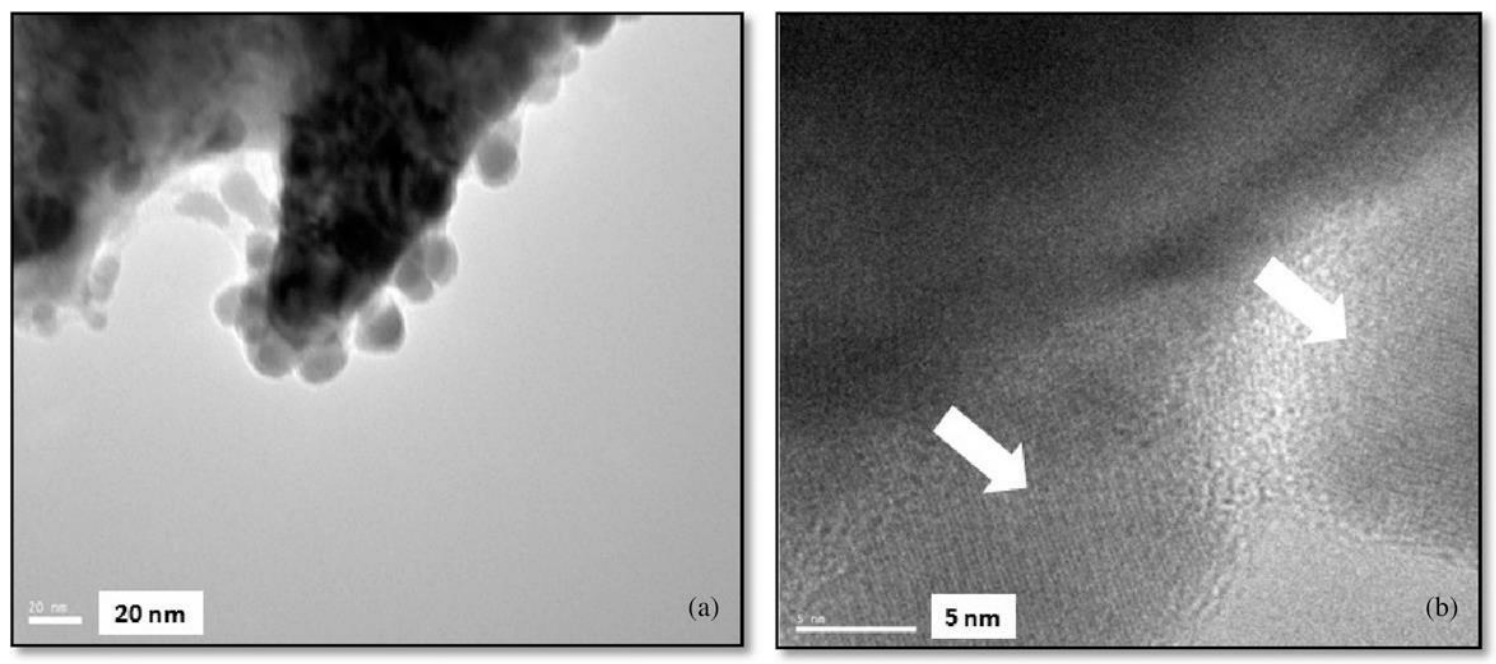

Figure 2. 4 (a) Transmission electron microscopy (TEM) image of copper particles on the carboxymethyl cellulose (CMC) structure (b) high-resolution TEM image of copper particles on the CMC structure.

\subsubsection{Antimicrobial properties of copper-carboxymethyl cellulose solution against nonpathogenic Escherichia coli DH5 $\alpha$}

The two photographs in Figure 2.5 show representative examples of the results for bacterial enumeration of the 18-h E. coli culture. Figure $2.5 \mathrm{a}$ is a $10^{-6}$ serial dilution showing too-numerous-to-count number of colonies, whereas Figure $2.5 \mathrm{~b}$ represents a $10^{-7}$ dilution of the 18 -h E. coli culture. Figure 2.5 b shows 34 colonies, and therefore, the procedure followed in this study to incubate E. coli resulted in a culture with $3.4 \times 10^{8}$ 
$\mathrm{CFU} / \mathrm{mL}$. The two photos in Figure 2.6 show representative examples for bacterial enumeration of the 18-h E. coli culture exposed to the $\mathrm{Cu}-\mathrm{CMC}$ solution for $10 \mathrm{~h}$ at $4{ }^{\circ} \mathrm{C}$. Both dilutions in Figure 2.6 show non-detectable E. coli survivors. Therefore, the 10-h exposure of $E$. coli to the $\mathrm{Cu}-\mathrm{CMC}$ solution in this study resulted in at least an 8-log microbial reduction. Both Petrifilms in Figure 2.6 show no colonies. Thus, the 10-h exposure of the 18-h $E$. coli culture to $\mathrm{Cu}-\mathrm{CMC}$ solution resulted in non-detectable levels of E. coli survivors. This represents at least an 8-log microbial reduction.
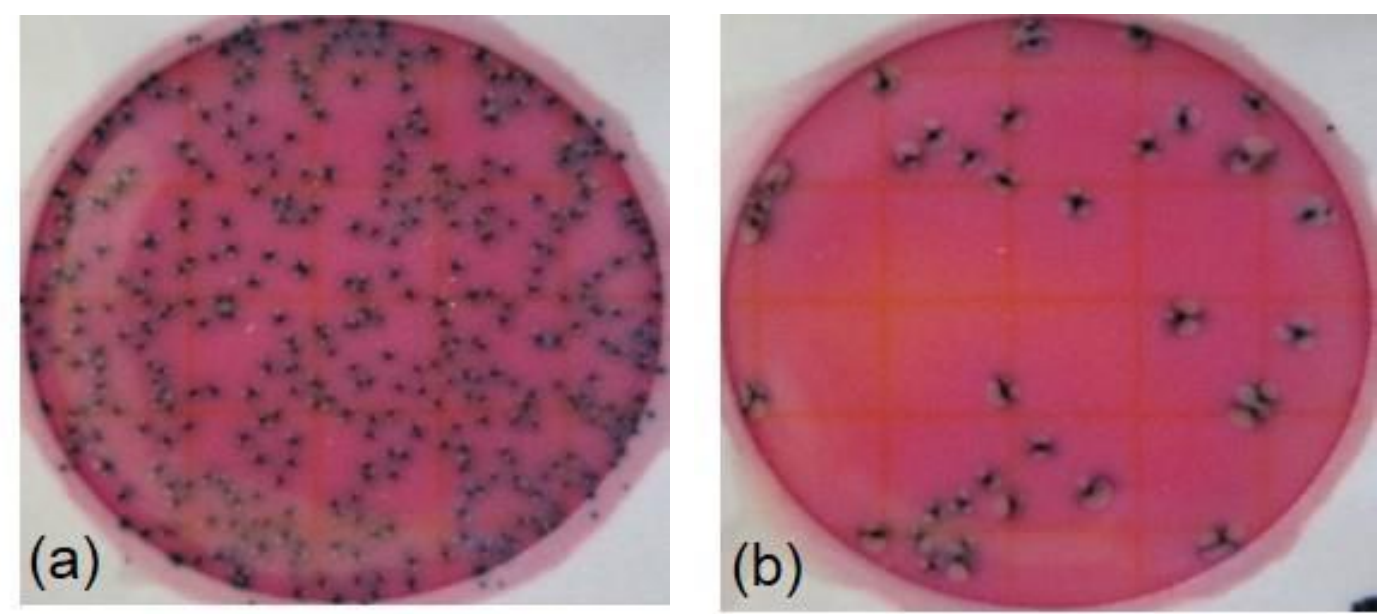

Figure 2. 5 Bacterial enumeration of the 18-h Escherichia coli culture. (a) Representative Petrifilm plate for $10^{-6}$ dilution with too-numerous-to-count number of colonies; (b) representative Petrifilm for $10^{-7}$ dilution with 34 colonies. Thus, the 18 -h E. coli culture contained $3.4 \times 10^{8}$ colony-forming units $/ \mathrm{mL}$.

Although an 8- $\log$ microbial reduction is encouraging, it needs to be emphasized that $E$. coli DH5 $\alpha$ is nonpathogenic with a high sensitivity to various stresses, unlike E. coli O157:H7, which is a typical foodborne pathogen with a higher resistance (Chalise et al 2007). E. coli DH5 $\alpha$ was used in this study as a nonpathogenic surrogate for the pathogenic E. coli $\mathrm{O} 157: \mathrm{H} 7$. The use of nonpathogenic bacterial surrogates is standard procedure in the initial determination of novel antimicrobial treatments such as in this study. Also, this study used E. coli DH5 $\alpha$ exposed to Cu-CMC solution and not the actual food packaging. However, a forthcoming study will include the determination of 
antimicrobial properties of copper nanoparticles in food packaging against foodborne pathogens such as E. coli $\mathrm{O} 157: \mathrm{H} 7$ and Salmonella spp.
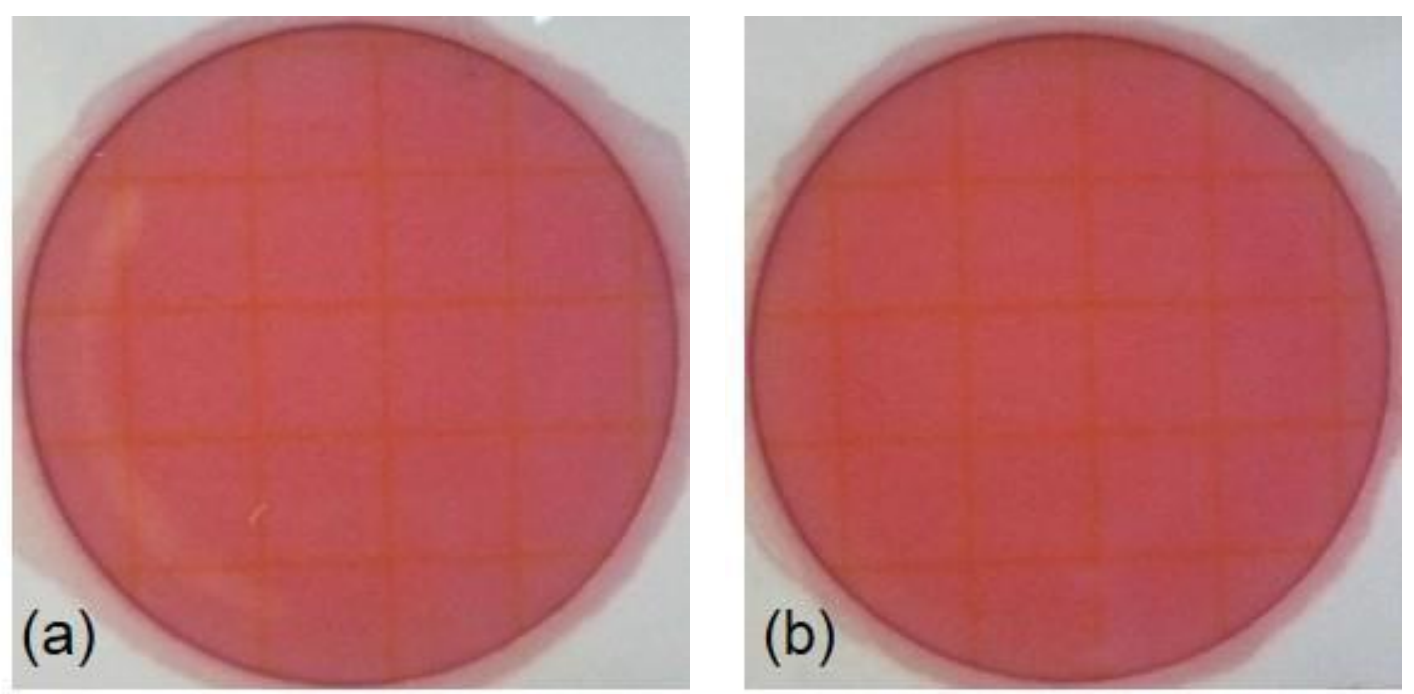

Figure 2. 6 Bacterial enumeration of the 18-h Escherichia coli culture exposed to coppercarboxymethyl cellulose solution for $10 \mathrm{~h}$ at $4{ }^{\circ} \mathrm{C}$. (a) Representative Petrifilm plate for $10^{-1}$ dilution. (b) Representative Petrifilm plate for $10^{-2}$ dilution.

\subsection{Conclusions}

$\mathrm{CMC}$ can be effectively used as a starting material to synthesize in situ $\mathrm{Cu}$ nanoparticles, and the final hybrid material has effective antimicrobial properties against the nonpathogenic surrogate foodborne pathogen E. coli $\mathrm{DH} 5 \alpha$.

\subsection{References}

Anyaogu, K. C., Fedorov, A. V., \& Neckers, D. C. (2008). Synthesis, characterization, and antifouling potential of functionalized copper nanoparticles. Langmuir, 24(8), 43404346. 
AOAC (1995) Official methods of analysis. 16th ed. Association of Official Analytical Chemists International, Gaithersburg, MD.

Black, J. L., \& Jaczynski, J. (2006). Temperature effect on inactivation kinetics of Escherichia coli O157: H7 by electron beam in ground beef, chicken breast meat, and trout fillets. Journal of Food Science, 71(6), M221-M227.

Cady, N. C., Behnke, J. L., \& Strickland, A. D. (2011). Copper-Based Nanostructured Coatings on Natural Cellulose: Nanocomposites Exhibiting Rapid and Efficient

Inhibition of a Multi-Drug Resistant Wound Pathogen, A. baumannii, and Mammalian Cell Biocompatibility In Vitro. Advanced Functional Materials, 21(13), 2506-2514.

Chalise, P. R., Hotta, E., Matak, K. E., \& Jaczynski, J. (2007). Inactivation kinetics of Escherichia coli by pulsed electron beam. Journal of Food Science, 72(7), M280M285.

Cioffi, N., Torsi, L., Ditaranto, N., Tantillo, G., Ghibelli, L., Sabbatini, L., ... \& Traversa, E. (2005). Copper nanoparticle/polymer composites with antifungal and bacteriostatic properties. Chemistry of Materials, 17(21), 5255-5262.

Cushen, M., Kerry, J., Morris, M., Cruz-Romero, M., \& Cummins, E. (2012). Nanotechnologies in the food industry-Recent developments, risks and regulation. Trends in Food Science \& Technology, 24(1), 30-46.

Duncan, T. V. (2011). Applications of nanotechnology in food packaging and food safety: barrier materials, antimicrobials and sensors. Journal of Colloid and Interface Science, 363(1), 1-24.

Ferreira, T., \%Rasband, W. (2012) ImageJ user guide IJ 1.46r. National Institutes of Health, Bethesda, MD. 193 pp. http://imagej.nih.gov/ij/docs/guide/ (14 August 2012). 
Gabbay, J., Borkow, G., Mishal, J., Magen, E., Zatcoff, R., \& Shemer-Avni, Y. (2006). Copper oxide impregnated textiles with potent biocidal activities. Journal of Industrial Textiles, 35(4), 323-335.

Hostynek, J. J., \& Maibach, H. I. (2004). Copper hypersensitivity: dermatologic aspects*. Dermatologic Therapy, 17(4), 328-333.

Hu, Y. H., Dang, W., Liu, C. S., \& Sun, L. (2010). Analysis of the effect of copper on the virulence of a pathogenic Edwardsiella tarda strain. Letters in Applied Microbiology, 50(1), 97-103.

Jia, B., Mei, Y., Cheng, L., Zhou, J., \& Zhang, L. (2012). Preparation of copper nanoparticles coated cellulose films with antibacterial properties through one-step reduction. ACS Applied Materials \& Interfaces, 4(6), 2897-2902.

Kanninen, P., Johans, C., Merta, J., \& Kontturi, K. (2008). Influence of ligand structure on the stability and oxidation of copper nanoparticles. Journal of Colloid and Interface Science, 318(1), 88-95.

Kotelnikova, N., Vainio, U., Pirkkalainen, K., \& Serimaa, R. (2007, August). Novel Approaches to Metallization of Cellulose by Reduction of Cellulose-Incorporated Copper and Nickel Ions. In Macromolecular Symposia (Vol. 254, No. 1, pp. 74-79).

\section{WILEY-VCH Verlag.}

Levanduski, L., \& Jaczynski, J. (2008). Increased resistance of Escherichia coli O157: H7 to electron beam following repetitive irradiation at sub-lethal doses. International Journal of Food Microbiology, 121(3), 328-334. 
Llorens, A., Lloret, E., Picouet, P. A., Trbojevich, R., \& Fernandez, A. (2012). Metallicbased micro and nanocomposites in food contact materials and active food packaging. Trends in Food Science \& Technology, 24(1), 19-29.

Mallick, S., Sharma, S., Banerjee, M., Ghosh, S. S., Chattopadhyay, A., \& Paul, A. (2012). Iodine-stabilized $\mathrm{Cu}$ nanoparticle chitosan composite for antibacterial applications. ACS Applied Materials \& Interfaces, 4(3), 1313-1323.

Mary, G., Bajpai, S. K., \& Chand, N. (2009). Copper (II) ions and copper nanoparticles-loaded chemically modified cotton cellulose fibers with fair antibacterial properties. Journal of Applied Polymer Science, 113(2), 757-766.

Nadagouda, M. N., \& Varma, R. S. (2007). Synthesis of thermally stable carboxymethyl cellulose/metal biodegradable nanocomposites for potential biological applications. Biomacromolecules, 8(9), 2762-2767.

Padalkar, S., Capadona, J. R., Rowan, S. J., Weder, C., Won, Y. H., Stanciu, L. A., \& Moon, R. J. (2010). Natural biopolymers: novel templates for the synthesis of nanostructures. Langmuir, 26(11), 8497-8502.

Pang, H., Gao, F., \& Lu, Q. (2009). Morphology effect on antibacterial activity of cuprous oxide. Chemical Communications, (9), 1076-1078.

Perelshtein, I., Applerot, G., Perkas, N., Wehrschuetz-Sigl, E., Hasmann, A., Guebitz, G., \& Gedanken, A. (2009). CuO-cotton nanocomposite: formation, morphology, and antibacterial activity. Surface and Coatings Technology, 204(1), 54-57.

Pinto, R. J., Neves, M. C., Neto, C. P., \& Trindade, T. (2012). Growth and chemical stability of copper nanostructures on cellulosic fibers. European Journal of Inorganic Chemistry, 2012(31), 5043-5049. 
Ren, G., Hu, D., Cheng, E. W., Vargas-Reus, M. A., Reip, P., \& Allaker, R. P. (2009). Characterisation of copper oxide nanoparticles for antimicrobial applications. International Journal of Antimicrobial Agents, 33(6), 587-590.

Ruparelia, J. P., Chatterjee, A. K., Duttagupta, S. P., \& Mukherji, S. (2008). Strain specificity in antimicrobial activity of silver and copper nanoparticles. Acta Biomaterialia, 4(3), 707-716.

Tesfai, A. T., Beamer, S. K., Matak, K. E., \& Jaczynski, J. (2011). Radioresistance development of DNA repair deficient Escherichia coli DH5 $\alpha$ in ground beef subjected to electron beam at sub-lethal doses. International Journal of Radiation Biology, 87(6), 571-578. 


\section{CHAPTER 3: NANOFIBRILLATED CELLULOSE AND COPPER NANOPARTICLES EMBEDDED IN POLYVINYL ALCOHOL FILMS FOR ANTIMICROBIAL APPLICATION}

\subsection{Chapter abstract}

Our long-term goal is to develop a hybrid cellulose-copper nanoparticle material as a functional nanofiller to be incorporated in thermoplastic resins for efficiently improving their antimicrobial properties. In this study, copper nanoparticles were first synthesized through chemical reduction of cupric ions on TEMPO nanofibrillated cellulose (TNFC) template using borohydride as a copper reducing agent. The resulting hybrid material was embedded into a polyvinyl alcohol (PVA) matrix using a solvent casting method. The morphology of TNFC-copper nanoparticles was analyzed by transmission electron microscopy (TEM); spherical copper nanoparticles with average size of $9.2 \pm 2.0 \mathrm{~nm}$ were determined. Thermogravimetric analysis and antimicrobial performance of the films were evaluated. Slight variations in thermal properties between the nanocomposite films and PVA resin were observed. Antimicrobial analysis demonstrated that one-week

exposure of nonpathogenic Escherichia coli DH5 $\alpha$ to the nanocomposite films results in up to 5-log microbial reduction.

\subsection{Introduction}

Currently a great amount of low-value, low-quality hardwood were generated in the Appalachian region, which can potentially be used as feedstock for novel bioproducts. Merely in West Virginia there is 2.41 million of dry tons of underutilized wood being produced each year, these low-quality and low-value wood might be a great source for nanocellulose production. Today the nanotechnology is opening a variety of possibilities of isolating and obtaining biopolymers from wood on the nanoscale. It has proved to be successful; specific applications for these novel raw materials are still a challenge. Based on our preliminary results (Zhong et al. 2013), one interesting application is the 
utilization of micro- and nanostructures of cellulose as templates and stabilizers for biocide nanoparticles with emphasis on the application as antimicrobial nanocomposites in the packaging and/or medical industry.

Copper, silver and gold are the most common metal antimicrobial materials that can be incorporated as nanomaterials in thermoplastic films for packaging and/or medical industry (Del Nobile et al. 2004; Zhang et al. 2008; Llorens et al. 2012); however, to avoid leaching, to improve metal dispersion, and to improve the contact between the metal and the bacterial wall a supportive material might be required. In addition, it is expected that the metal ions can be released from the film in a controlled way to effectively inhibit microbial growth. To date, the development of nanocomposite films fabricated from thermoplastic resins with cellulose nanofibers has emerged as a potentially effective approach for improving mechanical properties of these films (Liu et al. 2012; Liu et al. 2013). Our own preliminary findings provide evidence that these cellulose nanofibers could be used as support materials for copper nanoparticles, in turn, copper nanoparticles can endow the hybrid materials with the antimicrobial properties.

In general, the prevailing concept of grafting metal on the surface of cellulose derivatives includes coordinating the metallic cations via electrostatic interactions with negatively charged groups (e.g., carboxylate and hydroxyl) on the cellulose backbones. The presence of carboxyl groups on the cellulose backbone will help stabilize and reduce copper ions on the cellulosic structure. This research is primarily focused on the utilization of TEMPO-oxidized cellulose nanofibers as nano-sized cellulose for the synthesis of copper nanoparticles. The antimicrobial and thermal performances of the thermoplastic composites that incorporated resulting hybrid material were evaluated.

2,2,6,6-tetramethylpiperidine-1-oxyl (TEMPO) molecule is a highly stable nitroxyl radical which is used extensively in the selective oxidation of primary alcohols to corresponding aldehydes and carboxylic acids (Isogai et al. 2011). In aqueous environments, TEMPO catalyzes the conversion of carbohydrate primary alcohols to 
carboxylate (COO-) functionalities in the presence of a primary oxidizing agent, for example, sodium hypochlorite $(\mathrm{NaOCl})$. Different TEMPO-mediated oxidation reactions of mono-, oligo-, and polysaccharides for regioselective conversion of primary hydroxyls to carboxylate groups have been published elsewhere (de Nooy et al. 1995; Bragd et al. 2004). In particular, wood celluloses can be converted to individual nanofibers $3-4 \mathrm{~nm}$ wide with several microns length by TEMPO-mediated oxidation and successive mild disintegration in water (Fukuzumi et al. 2009; Isogai et al. 2011; Rodionova et al. 2012). During this reaction significant amounts of C6 carboxylate groups are selectively formed on each cellulose microfibril surface without any changes to the original crystallinity or crystal width of cellulose from woody biomass.

Polyvinyl alcohol (PVA) is a commercial important water-soluble, semicrystalline, transparent, biocompatible, and biodegradable polymer (Liu et al. 2013). It has been successfully blended with several natural materials such as fibers for PVA mechanical properties improvements. The increasing interest in the use of biodegradable and sustainable plastics is fueling the applications of PVA-based materials in several applications such as tissue scaffolding, filtration materials, and membranes and drug delivery (Ogur 2005; Peresin et al. 2010; Liu et al. 2013).

As mentioned previously, in this preliminary work a simple method was developed to produce hybrids of TEMPO nanofibrillated cellulose (TNFC) and copper nanoparticles. The hybrid material was subsequently embedded in polyvinyl alcohol thermoplastic resin and the final films were produced using a solvent casting method. The films were evaluated in terms of their morphology and thermal and antimicrobial properties.

\subsection{Materials and methods}

\subsubsection{Materials}


TEMPO nanofibrillated cellulose (TNFC) $(0.96$ wt.\%) from the Forest Product

Laboratory, Madison, WI; technical-crystal cupric sulfate pentahydrate $\left(\mathrm{CuSO}_{4} \cdot 5 \mathrm{H}_{2} \mathrm{O}\right)$ from Fisher Scientific, USA; sodium borohydride $\left(\mathrm{NaBH}_{4}\right)(0.5 \mathrm{M})$ from Acros Organics, USA; poly(vinyl alcohol) (99-100\% hydrolyzed, approx. M.W. 86000) was from Acros

Organics, USA; nonpathogenic E. coli DH5 $\alpha$ from Invitrogen Inc.,USA ( $\alpha$ substrain of DH5 described by Hanahan in 1985, "DH" stands for Douglas Hanahan) is a very sensitive microorganism. This E. coli contains mutations of the recA and gyrA (gyrase subunit A) genes that are necessary for DNA repair and replication. Therefore, $\operatorname{recA}$ and gyrA mutants have impaired ability to repair and recombine their DNA strands making the mutants sensitive to any stress including chemicals. This is why this sensitive and nonpathogenic E. coli was selected as a model microbial target in this study. Sterile trypticase soy broth (TSB) from Becton Dickinson, USA; Petrifilm E. coli/Coliform count plate from 3M, USA; Butterfield phosphate buffer from Hardy Diagnostics, USA.

\subsubsection{Preparation of the hybrid TNFC-copper nanoparticles}

Hybrid TNFC-copper nanoparticles were prepared by introducing copper nanoparticles on TNFC substrate by the chemical reduction of cupric ions. Twelve grams of TNFC gel containing 0.96 wt.\% cellulose nanofibers was dissolved in the deionized water under vigorous magnetic stirring. A predefined amount of cupric sulfate $\left(\mathrm{CuSO}_{4}\right)$ solution $(0.1$ $\mathrm{mol} / \mathrm{L}$ ) as shown in Table 3.1 was added by drops into the TEMPO-oxidized cellulose nanofibers solution. The mixture of TNFC and cupric sulfate was subjected to high-speed mixing while the cupric sulfate solution was added. Then the mixture was allowed to react at room temperature for $3 \mathrm{~h}$. After that, cupric ions were reduced to metallic copper or cuprous oxide nanoparticles by adding the predefined amount of reducing agent sodium borohydride $(0.5 \mathrm{~mol} / \mathrm{L})$. 
Table 3. 1 Preparation of the hybrid TNFC-copper nanoparticles.

\begin{tabular}{cccccc}
\hline Sample & $\begin{array}{c}\text { TNFC gel } \\
(\mathrm{g})\end{array}$ & $\begin{array}{c}\mathrm{TNFC} \\
(\mathrm{g})\end{array}$ & $\begin{array}{c}\mathrm{CuSO}_{4} \\
(0.1 \mathrm{~mol} / \mathrm{L}) \\
(\mathrm{mL})\end{array}$ & $\begin{array}{c}\mathrm{NaBH}_{4} \\
(0.5 \mathrm{~mol} / \mathrm{L}) \\
(\mathrm{mL})\end{array}$ & $\begin{array}{c}\text { Total Amount } \\
(\mathrm{mL})\end{array}$ \\
\hline $\begin{array}{c}\text { TNFC-copper } \\
\text { nanoparticles }(1) \\
\text { TNFC-copper } \\
\text { nanoparticles (2) } \\
\text { TNFC-copper }\end{array}$ & 12 & 0.1152 & 6.4 & 2.56 & 30 \\
$\begin{array}{c}\text { nanoparticles (3) } \\
\text { TNFC-copper }\end{array}$ & 12 & 0.1152 & 8.0 & 3.2 & 30 \\
nanoparticles (4) & 12 & 0.1152 & 11.2 & 4.42 & 30 \\
\hline
\end{tabular}

\subsubsection{Preparation of PVA/TNFC and PVA/TNFC-copper nanoparticles nanocomposite films}

Polyvinyl alcohol/TNFC-copper nanoparticle nanocomposite films were prepared by solvent casting method described elsewhere (Cheng et al. 2007). Ninety milliliters of deionized water was heated to $90{ }^{\circ} \mathrm{C}$ using a hot plate. Upon the desired temperature, $10 \mathrm{~g}$ of PVA was added into the hot water under vigorous magnetic stirring, after all PVA was added; the beaker will be covered; the mixture was heated at $90{ }^{\circ} \mathrm{C}$ for $2 \mathrm{~h}$. Subsequently, hybrid TNFC-copper nanoparticles solution was added by drops into clear PVA solution under vigorous magnetic stirring for $2 \mathrm{~h}$. The resulting solution was transferred to glass dish and put in the desiccator to degas for $24 \mathrm{~h}$ under vacuum and then put in the oven at $50{ }^{\circ} \mathrm{C}$ for $24 \mathrm{~h}$. PVA films with different copper concentration were formed and the compositions of the film were shown in Table 3.2. According to the weight ratio of copper in the composites, that is, 0.4, 0.5, 0.6, and $0.7 \mathrm{wt} . \%$, the nanocomposite films were coded as PVA/TNFC-Cu0.4, PVA/TNFC-Cu0.5, PVA/TNFC-Cu0.6, and PVA/TNFC-Cu0.7. Pure PVA and PVA/TNFC films were also prepared as control for antimicrobial testing. 
Table 3. 2 Preparation of the PVA/TNFC-copper nanoparticles nanocomposite films.

\begin{tabular}{|c|c|c|c|c|}
\hline \multirow[b]{2}{*}{ Composition } & \multicolumn{4}{|c|}{ Sample Code } \\
\hline & $\begin{array}{r}\text { PVA/ } \\
\text { TNFC- } \\
\text { Cu0.4 }\end{array}$ & $\begin{array}{l}\text { PVA/ } \\
\text { TNFC- } \\
\text { Cu0.5 }\end{array}$ & $\begin{array}{c}\text { PVA/ } \\
\text { TNFC- } \\
\text { Cu0.6 }\end{array}$ & $\begin{array}{c}\text { PVA/ } \\
\text { TNFC- } \\
\mathrm{Cu} 0.7\end{array}$ \\
\hline PVA $(\mathrm{g})$ & 10 & 10 & 10 & 10 \\
\hline TNFC-copper nanoparticles (1) (mL) & 30 & - & - & - \\
\hline TNFC-copper nanoparticles (2) (mL) & - & 30 & - & - \\
\hline TNFC-copper nanoparticles (3) (mL) & - & - & 30 & - \\
\hline TNFC-copper nanoparticles (4) (mL) & - & - & - & 30 \\
\hline $\begin{array}{l}\text { Copper content within final films } \\
\text { (wt.\%) }\end{array}$ & 0.4 & 0.5 & 0.6 & 0.7 \\
\hline
\end{tabular}

\subsubsection{Transmission electron microscopy (TEM)}

The morphology and particle size of copper nanoparticles on TNFC substrate were observed by JEOL TEM-2100 instrument (Tokyo, Japan) operating at $120 \mathrm{kV}$. TEM samples were typically prepared by dropping the hybrid TNFC-copper nanoparticles solution on a 200-mesh nickel grid coated with a carbon film.

\subsubsection{Thermogravimetric analyzer (TGA) characterization}

The thermal behaviors of pure PVA and its nanocomposite films were determined by TA Q50 thermogravimetric analyzer (Delaware, USA), with temperature ramp-up rate of 10 ${ }^{\circ} \mathrm{C} / \mathrm{min}$ while being purged with nitrogen at a flow rate of $20 \mathrm{~mL} / \mathrm{min}$. The sample weight was chosen between $3 \mathrm{mg}$ and $4 \mathrm{mg}$ for all of the samples tested.

\subsubsection{Determination of antimicrobial activity of PVA/TNFC-copper nanoparticles nanocomposite films}

E. coli lyfo-disks were reconstituted by crushing one pellet using a sterile spatula in 0.5 $\mathrm{mL}$ of sterile TSB. The content was aseptically transferred to $99.5 \mathrm{~mL}$ of sterile TSB and allowed to grow aerobically at $37^{\circ} \mathrm{C}$ for $24 \mathrm{~h}$ in an incubator/shaker set at $150 \mathrm{rpm}(\mathrm{C} 24$, 
New Brunswick Scientific, New Jersey, USA). This procedure yielded $100 \mathrm{~mL}$ of E. coli stock culture. For experiments, a loopful of the stock culture was transferred to $100 \mathrm{~mL}$ of sterile TSB followed by incubation at $37^{\circ} \mathrm{C}$ for $24 \mathrm{~h}$ in the $\mathrm{C} 24$ incubator/shaker set at $150 \mathrm{rpm}$. This procedure yielded a culture with appropriately $10^{8}$ colony forming units permilliliter $(\mathrm{CFU} / \mathrm{mL})$. A 2-mL aliquot of such culture was transferred to a surface of pure PVA, PVA/TNFC, and PVA/TNFC-copper nanoparticles films and incubated at room temperature for 1 week. To prevent excessive evaporation of the E. coli culture from the surface of films, the films were kept in an aerobic environment with saturated humidity. After 1-week exposure, $1 \mathrm{~mL}$ of $E$. coli culture was removed from the surface of films to enumerate $E$. coli survivors. Prior to removal, the $E$. coli culture on films was carefully mixed to obtain equal cell distribution. Enumeration was performed by a standard serial 10-fold dilution procedure and spread plating in a biosafety cabinet under aseptic procedures (Black and Jaczynski 2006; Levanduski and Jaczynski 2008). A 1-mL aliquot of the E. coli culture removed from the surface of films was aseptically mixed with $9 \mathrm{~mL}$ of diluent (Butterfield phosphate buffer) followed by shaking the diluent bottle to uniformly distribute bacterial cells. Subsequent serial 10- fold dilutions were aseptically made by taking $10 \mathrm{~mL}$ of diluted sample and transferring it to a $90 \mathrm{~mL}$ diluent bottle. Survivors were enumerated on selective medium (Petrifilm E. coli/Coliform Count Plate) using a standard spread-plating technique. A $1.0 \mathrm{~mL}$ aliquot of each serial 10 -fold dilution was pipetted and spread on 3M Petrifilm plates. The 3M Petrifilm plates were incubated at $35{ }^{\circ} \mathrm{C}$ for $48 \mathrm{~h}$ (AOAC 1995). Only plates with 15-150 colonies were counted. Experiments were independently triplicated $(n=3)$. Enumeration of $E$. coli survivors in each equipment was performed in duplicate. Mean values for $E$. coli survivors were used to calculate log reductions of E. coli on the tested films (Figure 4.5). Differences between treatments (i.e., different films) were tested using the Least Significant Difference (LSD) test. All statistical analyses of data were performed using JMP version 12 Statistical Software (Statistical Discovery, from SAS). 


\subsection{Results and discussion}

\subsubsection{Morphology of copper nanoparticles on TNFC template}
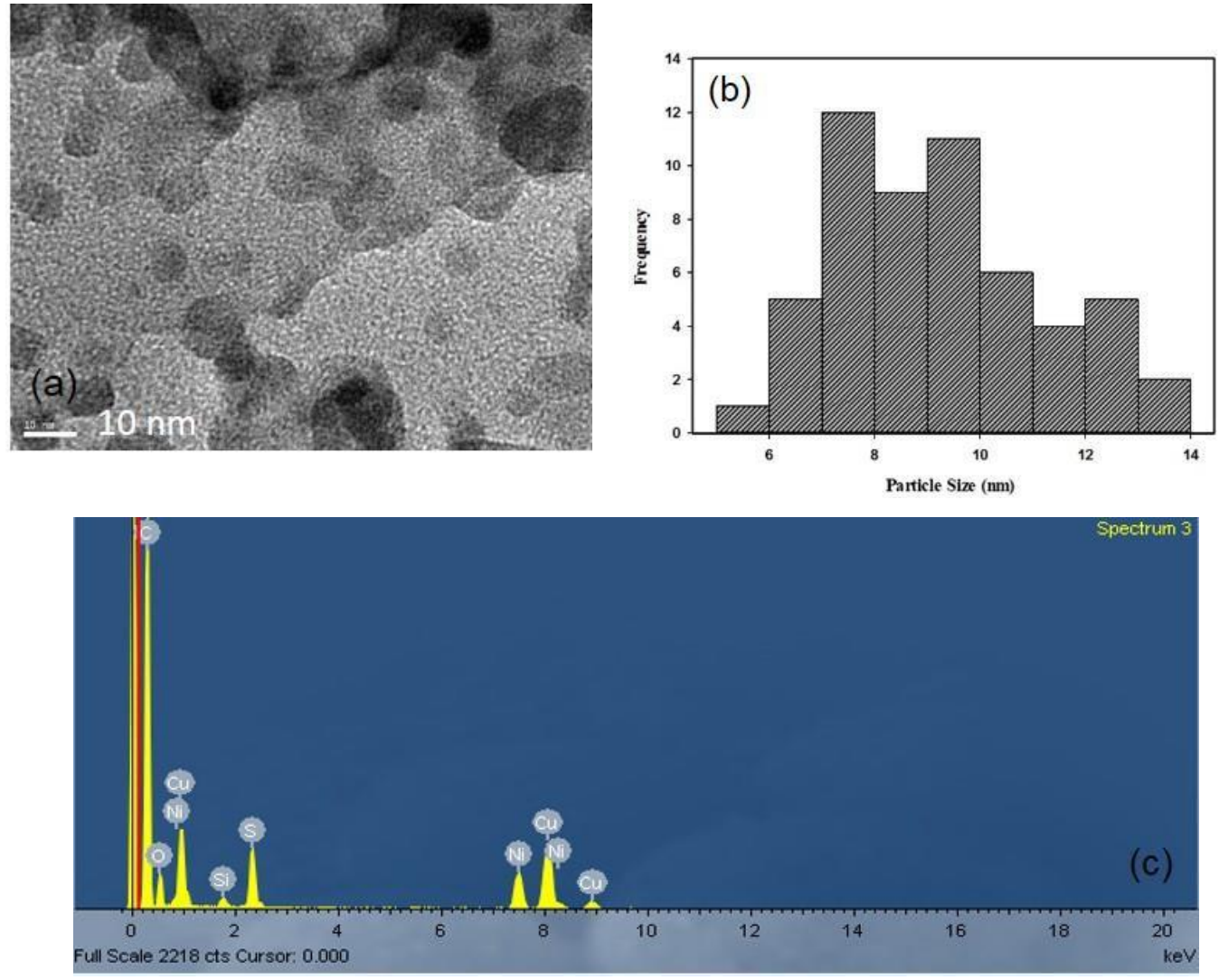

Figure 3. 1 (a) TEM images of hybrid TNFC-copper nanoparticles $($ Scale bar $=10 \mathrm{~nm})$; (b) histogram of particle size distribution ( $\mathrm{n}=55$ particles); (c) EDX spectrum of the hybrid TNFC-copper nanoparticles.

Figure 3.1 displays the TEM image of hybrid TNFC-copper nanoparticles (a), particle size histogram (b), and its corresponding EDX spectrum (c). Spherical copper nanoparticles with the particle size ranging from $5 \mathrm{~nm}$ to $14 \mathrm{~nm}$ and with average particles size $9.2 \pm 2.0 \mathrm{~nm}$ are observed. EDX confirms the formation of copper nanoparticles by exhibiting peaks at approximately $8 \mathrm{keV}$ (Figure 3.1c). 


\subsubsection{TGA analysis}
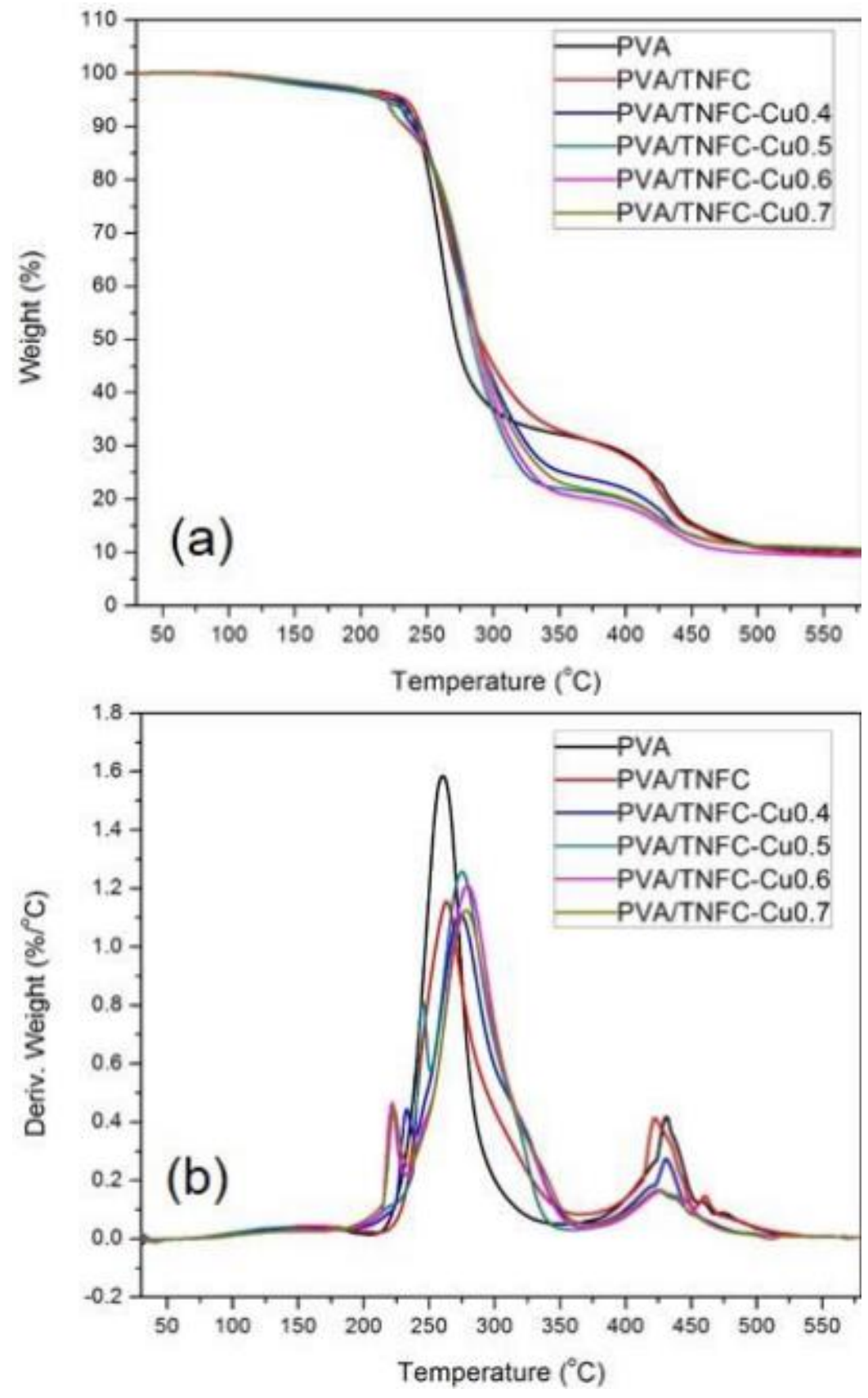

Figure 3. 2 (a) TGA curves for the pure PVA, PVA/TNFC film, and PVA/TNFC-Cu composite films; (b) the corresponding DTG curves. Peak temperatures $\left(T_{\max }\right)$ of PVA, PVA/TNFC, PVA/TNFC-Cu0.4, PVA/TNFC-Cu0.5, PVA/TNFC-Cu0.6, and PVA/TNFC-Cu0.7 were $260,263,274,275,278$, and $278^{\circ} \mathrm{C}$, respectively). 
Figure 3.2 shows typical TGA and DTG curves of the PVA composite films. All the PVA/TNFC-Cu composite films display four distinct weight loss stages at $30-210{ }^{\circ} \mathrm{C}$ as a result of loss of weakly physic-sorbed water, $210-230^{\circ} \mathrm{C}$ [decomposition of TNFCcopper nanoparticles nanocomposites, the thermal behavior of TNFC-copper nanoparticles is similar to that of carboxymethyl cellulose-copper nanoparticles reported by Nadagouda and Varma (2007)], 230-380 ${ }^{\circ} \mathrm{C}$ due to the decomposition of side chain of

PVA, and $380-550{ }^{\circ} \mathrm{C}$ as a consequence of the decomposition of main chain of PVA. Major weight losses were observed in the range of $210-550{ }^{\circ} \mathrm{C}$, which corresponded to the structural decomposition of PVA and thermal degradation of TNFC. $T_{\max }$ is the decomposition temperature corresponding to the maximum weight loss and relates to the

maximum decomposition rate. In Figure $3.2 \mathrm{~b}$, we can see that $T_{\max }$ of PVA/TNFC-Cu composite films shifted to higher temperature compared to that of pure PVA and PVA/TNFC; an increase of $T_{\max }$ was observed from $260{ }^{\circ} \mathrm{C}$ to $278{ }^{\circ} \mathrm{C}$ for PVA and PVA/TNFC-Cu0.7 composite film, respectively. The thermal decomposition of PVA/TNFC-Cu films shifted slightly toward high temperature, suggesting that the composite films had higher thermal stability, which can be attributed to the presence of copper nanoparticles embedded in the PVA matrix.

\subsubsection{Antimicrobial activity of PVA/TNFC-copper nanoparticles films}

The antimicrobial properties of pure PVA, PVA/TNFC films, and PVA/TNFC-copper nanoparticle films with different copper concentration were tested against $E$. coli. Figures 3.3-3.4 show representative images of visual examples of different serial 10-fold dilutions for E. coli survivors following their exposure to pure PVA and PVA/TNFC films (Figure 3.3) and PVA/TNFC-copper nanoparticle films with different copper concentration (Figure 3.4).

The initial concentration of E. coli (i.e., prior to exposure to films) was approximately $10^{8}$ $\mathrm{CFU} / \mathrm{mL}$. Figure 3.3 shows relatively minimal reduction of the initial $E$. coli concentration following exposure to pure PVA and PVA/TNFC films, while Figure 3.4 
shows a trend of increasing microbial reduction as a function of greater copper nanoparticles concentration in films. The counts from enumeration of E. coli survivors (representative images shown in Figures 3.3-3.4) were log converted and used to determine $E$. coli reductions as a function of exposure to different films (Figure 3.5).

Figure 3.5 shows that PVA and PVA/TNFC films resulted in similarly $(P>0.05)$ minimal reduction of $E$. coli.
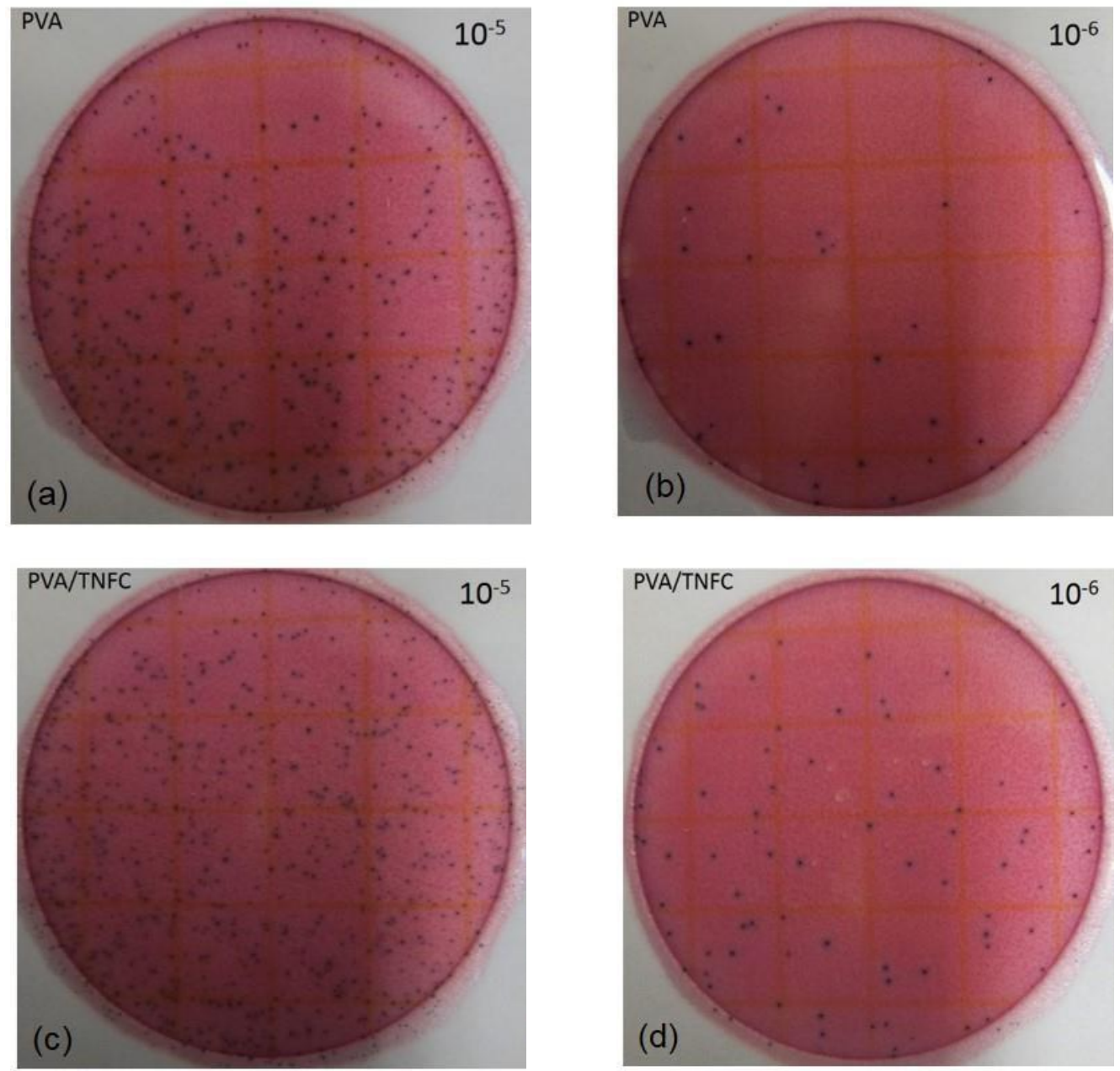

Figure 3. 3 Bacterial enumeration of the 24-h Escherichia coli culture exposed to control materials (a, b) PVA film and (c, d) PVA/TNFC film for 1 week at room temperature. (a, c) Representative Petrifilm plates for $10^{-5}$ dilution; (b, d) Representative Petrifilm co unt plates for $10^{-6}$ dilution. 
However, increasing concentration of copper nanoparticles in PVA/TNFC films resulted in greater $(P<0.05)$ reduction of E. coli. After 1-week exposure of E. coli to films containing $0.4 \mathrm{wt} . \%, 0.5 \mathrm{wt} . \%$, and $0.6 \mathrm{wt} . \%$ of copper nanoparticles, reduction of $E$. coli

has gradually increased $(P<0.05)$ and reached about 5-log reduction for the highest inclusion of copper nanoparticles in the film. Based on Figure 3.5, embedding copper nanoparticles in the PVA/TNFC film results in inactivation of $E$. coli that shows greater efficacy with higher concentration of copper nanoparticles. However, it needs to be emphasized that $E$. coli used in this study as a microbial target was a very sensitive strain; and, therefore, the antimicrobial efficacy of copper nanoparticles would likely be less profound for a typical foodborne pathogen such as E. coli O157:H7. Further research using more resistant foodborne pathogens is recommended. Even though the antimicrobial mechanism of copper nanoparticles against microorganisms has not been fully understood, three hypothetical mechanisms are the most widely accepted and reported in the literature: (1) copper nanoparticles accumulate in the bacterial membrane and cause changes in membrane permeability (Amro et al. 2000); (2) reactive oxygen species (ROS) produced through Fenton-type reactions lead to free-radical-mediated cellular damage (Santo et al. 2010; Chatterjee et al. 2014); (3) the release of copper ions from nanoparticles causes inactivation of enzymes and depletion of intracellular ATP as well as disruption of DNA replication (Macomber and Imlay 2009).

In this study we propose that the antimicrobial effect of PVA/TNFC-copper nanoparticles films is directly related to the transfer of copper ions leaching in a controlled manner from the PVA matrix to bacterial cells. This is consistent with the traditional hypothesis that metal ions attach to the negatively charged bacterial cell wall, resulting in disruption of cell wall permeability and thus inducing protein denaturation and finally cell death. PVA is a hydrophilic polymer; and, therefore, it is hygroscopic. Water uptake may induce the release of copper ions embedded in nanoparticles within the PVA matrix as a result of surface oxidation that occurs when copper nanoparticles are exposed to oxygen (Llorens et al. 2012). 

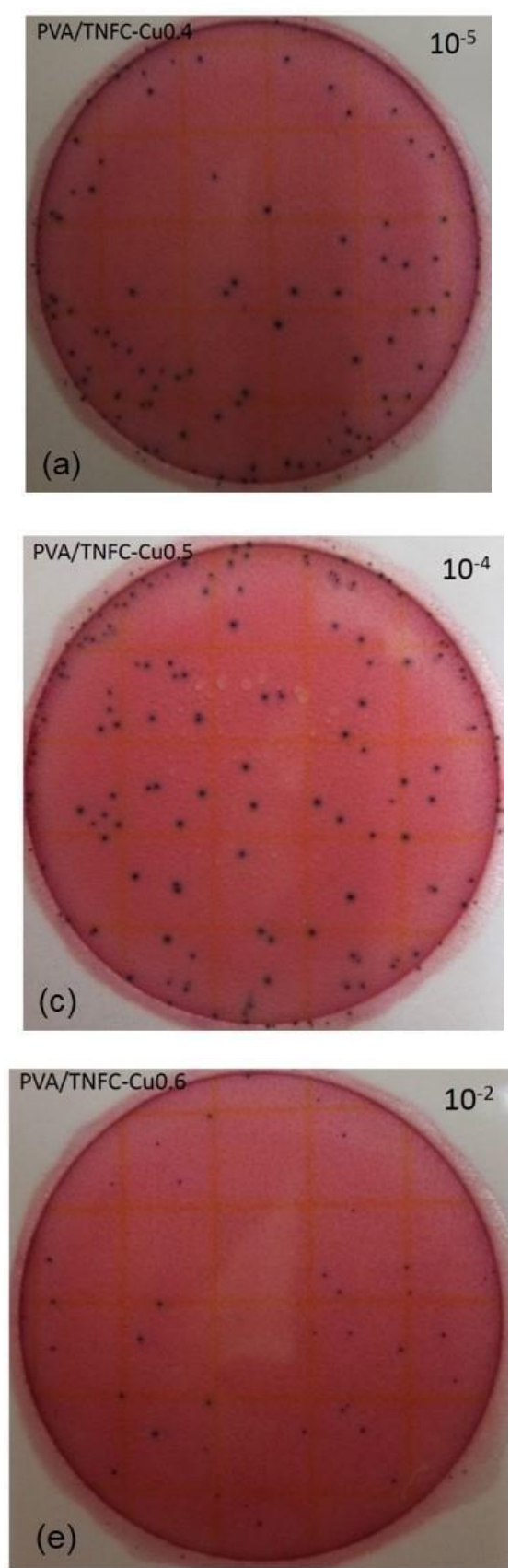
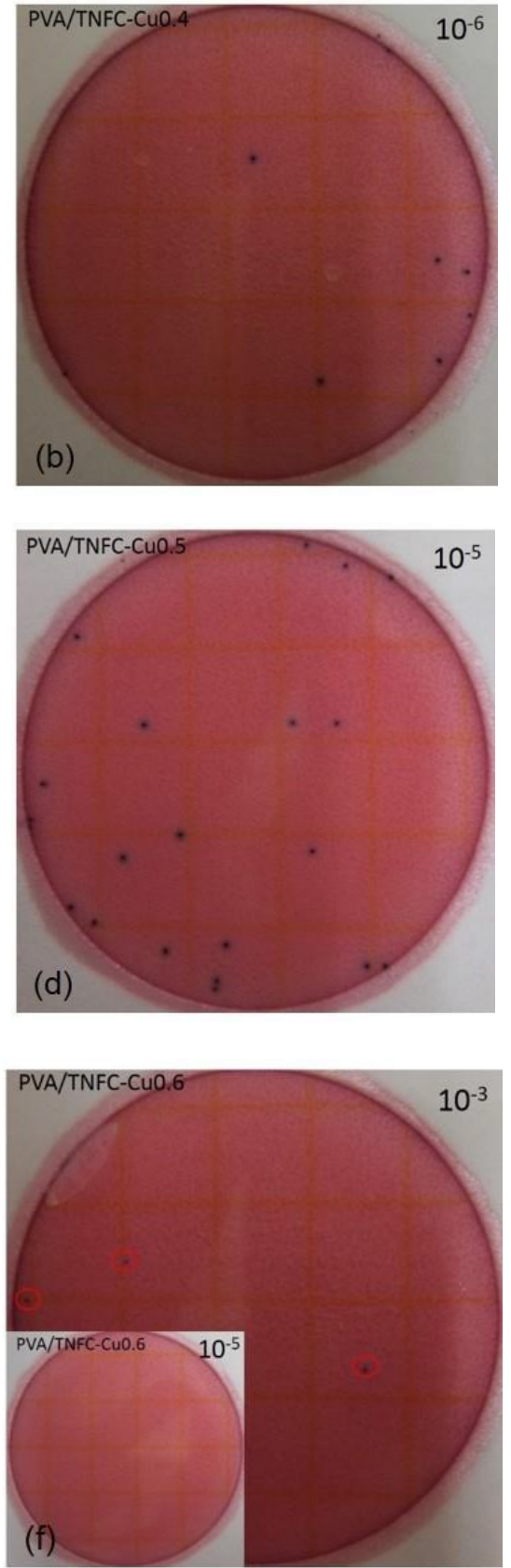

Figure 3. 4 Bacterial enumeration of the 24-h Escherichia coli culture exposed to PVA nanocomposite film containing various copper content (a, b) PVA/TNFC-Cu0.4, (c, d) PVA/TNFC-Cu0.5, and (e, f) PVA/TNFC-Cu0.6 films for 1 week at room temperature. (a-f) Representative Petrifilm count plates for various dilutions. 


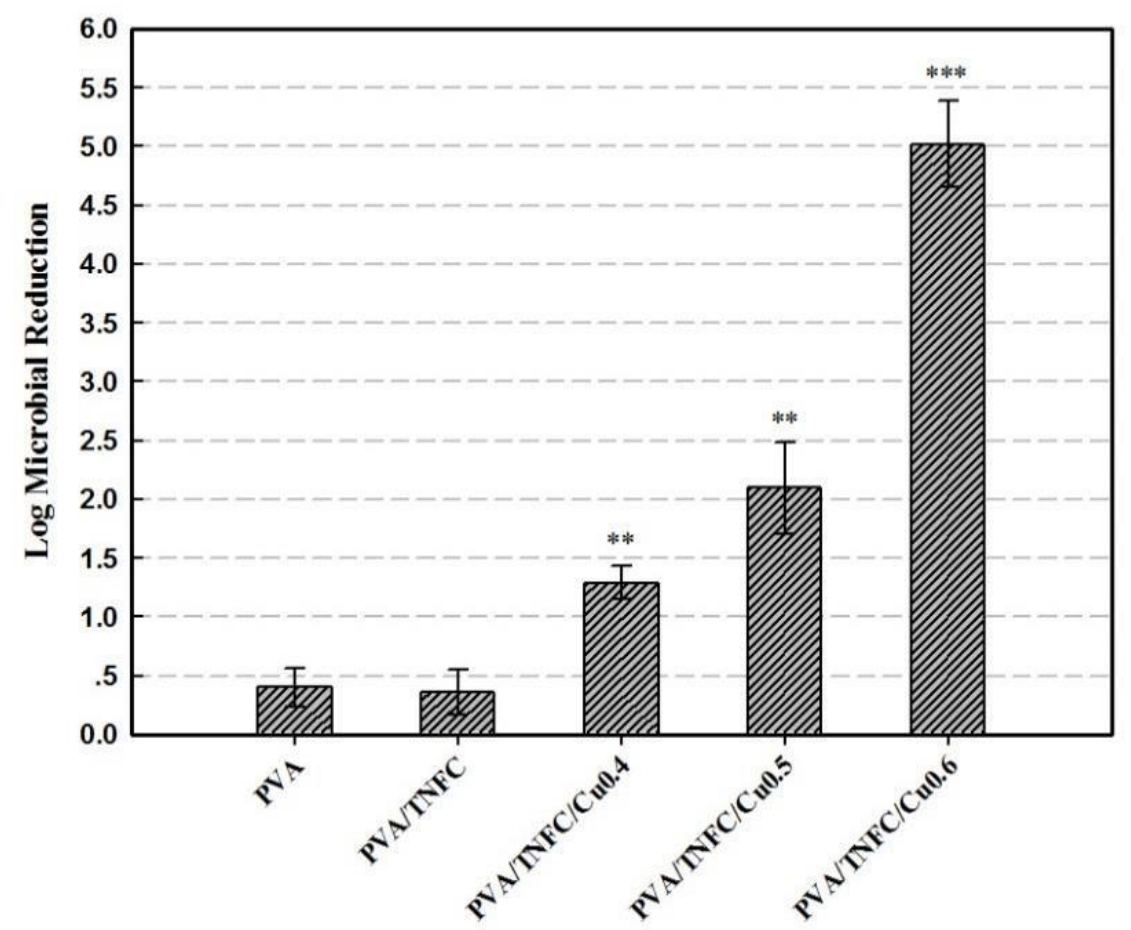

Figure 3. 5 Microbial reduction induced by PVA/TNFC-copper nanoparticles after 1week exposure. The Asterisks refer to significant levels compared to one of the controls: PVA/TNFC, $\mathrm{p}<0.05(*), \mathrm{p}<0.01(* *)$, and $\mathrm{p}<0.001(* * *)$.

\subsection{Conclusions}

Cuprous oxide nanoparticles with average diameter of $9.2 \pm 2.0 \mathrm{~nm}$ were successfully synthesized on the TEMPO nanofibrillated cellulose. The PVA composite films with TNFC-copper nanoparticles exhibited improved thermal stability, the decomposition temperature, corresponding to the maximum weight loss, increased from $260{ }^{\circ} \mathrm{C}$ for pure PVA, to $278{ }^{\circ} \mathrm{C}$ for the PVA composite. The incorporation of hybrid TNFC-copper nanoparticles within PAV matrix endows the resulting composite films with antimicrobial properties. The PVA film containing copper content up to $0.6 \mathrm{wt} \%$

exhibited a strong antimicrobial activity against $E$. coli $\mathrm{DH} 5 \alpha$, resulting in up to 5-log microbial reduction. The results suggest that TNFC-copper nanoparticles nanocomposites as antimicrobial nanofillers are valuable for PVA applications 


\subsection{References}

Amro, N. A., Kotra, L. P., Wadu-Mesthrige, K., Bulychev, A., Mobashery, S., \& Liu, G. Y. (2000). High-resolution atomic force microscopy studies of the Escherichia coli outer membrane: structural basis for permeability. Langmuir, 16(6), 2789-2796.

An, J., Zhang, M., Wang, S., \& Tang, J. (2008). Physical, chemical and microbiological changes in stored green asparagus spears as affected by coating of silver nanoparticlesPVP. LWT-Food Science and Technology, 41(6), 1100-1107.

AOAC (1995) Official methods of analysis. 16th ed. Association of Official Analytical Chemists International, Gaithersburg, MD.

Black, J. L., \& Jaczynski, J. (2006). Temperature effect on inactivation kinetics of Escherichia coli O157: H7 by electron beam in ground beef, chicken breast meat, and trout fillets. Journal of Food Science, 71(6), M221-M227.

Bragd, P. L., Van Bekkum, H., \& Besemer, A. C. (2004). TEMPO-mediated oxidation of polysaccharides: survey of methods and applications. Topics in Catalysis, 27(1-4), 4966.

Chatterjee, A. K., Chakraborty, R., \& Basu, T. (2014). Mechanism of antibacterial activity of copper nanoparticles. Nanotechnology, 25(13), 135101.

Cheng, Q., Wang, S., Rials, T. G., \& Lee, S. H. (2007). Physical and mechanical properties of polyvinyl alcohol and polypropylene composite materials reinforced with fibril aggregates isolated from regenerated cellulose fibers. Cellulose, 14(6), 593-602. 
Del Nobile, M. A., Cannarsi, M., Altieri, C., Sinigaglia, M., Favia, P., Iacoviello, G., \& d'Agostino, R. (2004). Effect of Ag-containing Nano-composite Active Packaging System on Survival of Alicyclobacillus acidoterrestris. Journal of Food Science, 69(8), E379-E383.

de Nooy, A. E., Besemer, A. C., \& van Bekkum, H. (1995). Selective oxidation of primary alcohols mediated by nitroxyl radical in aqueous solution. Kinetics and mechanism. Tetrahedron, 51(29), 8023-8032.

Fukuzumi, H., Saito, T., Iwata, T., Kumamoto, Y., \& Isogai, A. (2008). Transparent and high gas barrier films of cellulose nanofibers prepared by TEMPO-mediated oxidation. Biomacromolecules, 10(1), 162-165.

Isogai, A., Saito, T., \& Fukuzumi, H. (2011). TEMPO-oxidized cellulose nanofibers. Nanoscale, 3(1), 71-85.

Levanduski, L., \& Jaczynski, J. (2008). Increased resistance of Escherichia coli O157: H7 to electron beam following repetitive irradiation at sub-lethal doses. International Journal of Food Microbiology, 121(3), 328-334.

Liu, H., Song, J., Shang, S., Song, Z., \& Wang, D. (2012). Cellulose nanocrystal/silver nanoparticle composites as bifunctional nanofillers within waterborne polyurethane. ACS Applied Materials \& Interfaces, 4(5), 2413-2419.

Llorens, A., Lloret, E., Picouet, P. A., Trbojevich, R., \& Fernandez, A. (2012). Metallicbased micro and nanocomposites in food contact materials and active food packaging. Trends in Food Science \& Technology, 24(1), 19-29. 
Macomber, L., \& Imlay, J. A. (2009). The iron-sulfur clusters of dehydratases are primary intracellular targets of copper toxicity. Proceedings of the National Academy of Sciences, 106(20), 8344-8349.

Nadagouda, M. N., \& Varma, R. S. (2007). Synthesis of thermally stable carboxymethyl cellulose/metal biodegradable nanocomposites for potential biological applications. Biomacromolecules, 8(9), 2762-2767.

Ogur, O. (2005). Polyvinyl alcohol: materials, processing and application. Rapra Review Reports, 16(12), 1-130.

Peresin, M. S., Habibi, Y., Zoppe, J. O., Pawlak, J. J., \& Rojas, O. J. (2010). Nanofiber composites of polyvinyl alcohol and cellulose nanocrystals: manufacture and characterization. Biomacromolecules, 11(3), 674-681.

Rodionova, G., Eriksen, Ø., \& Gregersen, Ø. (2012). TEMPO-oxidized cellulose nanofiber films: effect of surface morphology on water resistance. Cellulose, 19(4), 1115-1123.

Santo, C. E., Morais, P. V., \& Grass, G. (2010). Isolation and characterization of bacteria resistant to metallic copper surfaces. Applied and Environmental Microbiology, 76(5), 1341-1348.

Zhong, T., Oporto, G. S., Jaczynski, J., Tesfai, A. T., \& Armstrong, J. (2013). Antimicrobial properties of the hybrid copper nanoparticles-carboxymethyl cellulose. Wood and Fiber Science, 45(2), 215-222. 


\section{CHAPTER 4: USING TWO CELLULOSIC TEMPLATES TO SYNTHESIZE COPPER NANOPARTICLES FOR ANTIMICROBIAL PURPOSES}

\subsection{Chapter abstract}

Copper nanoparticles were successfully synthesized on TEMPO nanofibrillated cellulose (TNFC) and on carboxymethyl cellulose (CMC). Polyvinyl alcohol (PVA) films containing the hybrid carboxylate cellulose-copper nanoparticles were prepared by solvent casting method. Both PVA films containing CMC-copper nanoparticles and TNFC-copper nanoparticles demonstrated improved antimicrobial performance against E.coli DH $5 \alpha$ compared with the pure PVA thermoplastic resin, and approximately $90 \%$ less cellulosic material was required when using TNFC as template compared to CMC template to get similar films antimicrobial performance. Only copper (II) was present on the surface of both PVA composite films and copper (I) was available in both films bulk material. PVA containing the hybrid CMC-copper nanoparticles inactivate more E.coli at the same exposure time when compared with PVA films containing hybrid TNFC-copper nanoparticles, that can be attributed to the presence of more crystalline $\mathrm{Cu}_{2} \mathrm{O}$ (copper (I)) in PVA/CMC matrix compared to the amount available in PVA/TNFC matrix. Copper ion release measurement revealed that there is not a direct relationship between copper ion release and film antimicrobial performance. The storage modulus, as an indicator of the films mechanical properties, increased up to $164 \%$ and $217 \%$ when CMC and TNFC hybrid materials were introduced into the PVA films. The decomposition temperatures,

corresponding to the maximum weight loss of PVA films, increased by $18{ }^{\circ} \mathrm{C}$ and $25^{\circ} \mathrm{C}$ over pure PVA when TNFC-copper nanoparticles and CMC-copper nanoparticles were added in the films, respectively. 


\subsection{Introduction}

Cellulosic micro- and nanomaterials have been effectively used to improve mechanical properties of several thermoplastic films (Chakraborty et al. 2006; Liu et al. 2013). The feasibility to improve not only mechanical properties, but also antimicrobial performance of those films will open novel opportunities of application that range from packing, medical, safety, etc. To date, some work has been performed introducing copper nanoparticles on cellulosic raw material with the aim to improve the antimicrobial properties of cellulose, but to the knowledge of the authors there are few work performed using this hybrid material in thermoplastics films for improving their antimicrobial activity (Zhong et al. 2015a). Coating copper nanoparticles on the surface of cellulose substrates resulted in composites with rapid and efficient antimicrobial performance against multidrug resistant pathogen A. Baumannii , S. aureus and E. coli (Cady et al. 2011; Jia et al. 2012). Metallic nanoparticles have been attracting important attention because of their large specific surface area and their novel physical and chemical properties at the nanoscale level (Gong et al. 2007). Particularly, metallic copper and copper oxides, have demonstrated effective antimicrobial activities against a broad spectrum of microorganisms, fungi and viruses (Borkow and Gabbay, 2009; Zhong et al. 2013). In contrast to the low sensitivity of human tissue to copper (Hostynek and Maibach 2004), prokaryotes (i.e., bacteria) are extremely susceptible to copper. In February 2008, the US Environmental Protection Agency approved the registration of copper alloys because they can inactivate certain bacteria associated with potentially fatal microbial infections (Llorens et al. 2012). The antimicrobial efficacy of copper have been confirmed against Escherichia coli O157:H7, Staphylococcus aureus, Enterobacter aerogenes, and Pseudomonas aeruginosa (Llorens et al. 2012). It is evident that copper has well-established antimicrobial properties and has great potential in the antimicrobial applications.

The high tendency to agglomerate limits the application of copper nanoparticles. The feasibility to use a template where copper can be synthesized in a uniform manner, avoiding aggregation is therefore highly desirable. The utilization of cellulosic raw material 
resulted effectively in protecting copper nanoparticles from aggregation and fast oxidation (Padalkar et al. 2010; Shi et al. 2012; Zhong et al. 2013). As presented by other research groups, the cellulosic material might act as an excellent stabilizer for the growth of copper nanoparticles (He et al. 2003). There are three general processes in which copper can be attached to cellulosic material are: 1) physical impregnation of copper into cellulose, 2) ion-dipole interaction and 3) electrostatic assembly. Perelshtein et al. (2009) for instance, used ultrasound irradiation to incorporate copper oxide nanoparticles at the surface of cellulose; He at al. (2003) anchored copper ions on ether and hydroxyl cellulosic sites via ion-dipole interactions, followed the reduction of copper ions by chemical reducing agents or UV irradiation; and Cady et al. (2011) grafted negatively charged functional groups, such as carboxylate, on cellulose. After that, they used cationic copper ions that were electrostatically attracted to the negatively charged surface of cellulose. In our research group a simple route to synthesize copper nanoparticles in aqueous solution and use an organic material as template has been successfully demonstrated (Zhong et al. 2013). The antimicrobial properties of the hybrid material were established; however, the performance of the hybrid material in thermoplastic resins for further applications remained as a gap and is the main goal of the present research.

The main objectives of this work consisted of evaluating the antimicrobial performance of polyvinyl alcohol (PVA) films after the incorporation of an hybrid material composed of a carbohydrate and copper nanoparticles. Likewise, to determine changes in the copper crystalline structure and state of oxidation that can be affecting the antimicrobial activities of the PVA films. In addition, copper release from PVA composite films containing hybrid cellulose-copper nanoparticles materials in lauryl tryptose broth (LTB) medium so as to investigate the relationship between copper ions release and antimicrobial performance. LTB was the food simulant to trigger the ion release from the films. Meantime, the evaluation of the films in terms of their thermal and mechanical characteristics was also performed. 
Because of unique properties of cellulosic templates, such as light weight, high strength, biodegradability, biocompatibility, and their carboxylic group availability, carboxymethyl cellulose (CMC) and 2,2,6,6-tetramethylpiperidine-1-oxyl radical (TEMPO) nanofibrillated cellulose (TNFC) was chose as a carbohydrate template to attach copper nanoparticles.

CMC is microcrystalline cellulose, which consists of aggregate bundles of cellulose microfibrils (Moon et al. 2011) and it is commercially available with particle sizes ranging from $10-50 \mu_{\mathrm{m}}$ diameter and degree of polymerization ranging from 400 to 3200 . TNFC suspension consists of cellulose fibrils in water; these fibrils' width range from 4 to $20 \mathrm{~nm}$ and length between 500-2000 nm (Moon et al. 2011). Depending on the TEMPO oxidation conditions, such as $\mathrm{pH}$ and the concentration of $\mathrm{NaClO}$ used in the reaction, TNFC has a degree of polymerization ranging from 200 to 1200 (Saito and Isogai, 2004; Isogai et al. 2011; Moon et al. 2011).

The hybrid cellulose-copper nanoparticle materials were incorporated into polyvinyl alcohol (PVA) resin using a simple solvent casting method. PVA is one of the most commercially important synthetic water-soluble, semicrystalline, nontoxic, transparent, biocompatible and biodegradable polymer (Liu et al. 2013). The increasing interest in the use of biodegradable and sustainable plastics sparked many uses of PVA in the applications such as tissue scaffolding, filtration materials, membranes and drug delivery, etc (Liu et al. 2013).

\subsection{Materials and methods}

\subsubsection{Materials}

TEMPO nanofibrillated cellulose (TNFC) gel (0.96 wt.\% TNFC in water and $1.24 \mathrm{mmol}$ $\mathrm{COONa} / \mathrm{g}$ dry TNFC) from the Forest Product Laboratory, Madison, WI; sodium carboxymethyl cellulose (Molecular weight: 90,000) (NaCMC) from Sigma Aldrich, USA; technical-crystal cupric sulfate pentahydrate $\left(\mathrm{CuSO}_{4} \cdot 5 \mathrm{H}_{2} \mathrm{O}\right)$ from fisher Scientific, USA; 
sodium borohydride $\left(\mathrm{NaBH}_{4}\right)$ (0.5 M) and poly(vinyl alcohol) (99-100\% hydrolyzed, approx. M.W. 86000) from Acros Organics, USA; Escherichia coli DH5 $\alpha$ from Invitrogen Inc., USA; lauryl tryptose broth (LTB) from Becton Dickinson, USA; Petrifilm E. coli/Coliform count plate from 3M, USA; butterfield phosphate buffer from Hardy Diagnostics, USA; nonpathogenic E.coli DH5 $\alpha$ from Invitrogen Inc.,USA ( $\alpha$ substrain of DH5 described by Hanahan in 1985, "DH" stands for Douglas Hanahan) is a very sensitive microorganism. This E. coli contains mutations of the recA and gyrA (gyrase subunit A) genes that are necessary for DNA repair and replication. Therefore, recA and gyrA mutants have impaired ability to repair and recombine their DNA strands making the mutants sensitive to any stress including chemicals. This is why this sensitive and nonpathogenic E. coli was selected as a model microbial target in this study.

\subsubsection{Preparation of the hybrid TNFC-copper nanoparticles}

Hybrid TNFC-copper nanoparticles was prepared by introducing copper nanoparticles on TNFC substrate by the chemical reduction of cupric ions. Twelve grams of TNFC gel containing $0.96 \mathrm{wt} \%$ TNFC was dissolved in the deionized water under vigorous magnetic stirring. A 9.6-mL aliquot of $\mathrm{CuSO}_{4}$ solution $(0.1 \mathrm{~mol} / \mathrm{L})$ was added by drops into the TNFC solution. The mixture of TNFC and cupric sulfate was subjected to high-speed mixing while the cupric sulfate solution was added. Then the mixture was allowed to react at room temperature for $3 \mathrm{~h}$. After that, cupric ions were reduced to metallic copper or copper oxide nanoparticles by adding the $1.92 \mathrm{~mL}$ of reducing agent sodium borohydride $(0.5 \mathrm{~mol} / \mathrm{L})$.

\subsubsection{Preparation of the hybrid CMC-copper nanoparticles}

The general procedure used for the preparation of the hybrid CMC-copper nanoparticles has been described previously (Zhong et al. 2013). A 10.5-mL aliquot of cupric sulfate solution $(0.1 \mathrm{~mol} / \mathrm{L})$ was added by drops into the CMC solutions. The mixtures of CMC and cupric sulfate were subjected to high-speed mixing while the cupric sulfate solution was added. 
Then the mixtures were allowed to react at room temperature for $3 \mathrm{~h}$. After that, cupric ions were reduced to metallic copper or copper oxide nanoparticles by adding $2.1 \mathrm{~mL}$ of reducing agent sodium borohydride.

\subsubsection{Preparation of PVA/TNFC-copper nanoparticles and PVA/CMC-copper nanoparticles films}

Polyvinyl alcohol/TNFC-copper nanoparticle and PVA/CMC-copper nanoparticles films were prepared by solvent casting method described by Cheng et al. (2007). A 90-mL aliquot of deionized water was heated to $90{ }^{\circ} \mathrm{C}$. Upon the desired temperature, $10 \mathrm{~g}$ of PVA was added to the hot water under vigorous magnetic stirring, after all PVA was added, the beaker will be covered, the mixture was heated at $90{ }^{\circ} \mathrm{C}$ for $2 \mathrm{~h}$. Subsequently, resulting cellulose-copper nanoparticles solution was added by drops into clear PVA solution under vigorous magnetic stirring for $2 \mathrm{~h}$. The resulting solution was transferred to glass dish and put in the desiccator to degas for $24 \mathrm{~h}$ under vacuum, and then put in the oven at $40{ }^{\circ} \mathrm{C}$ for 24 h. PVA films containing TNFC-copper nanoparticles or CMC-copper nanoparticles were formed, respectively. According to the weight percentage of copper to the total weight of PVA, cellulose and copper, i.e., $0.6 \mathrm{wt} \%$, and the type of cellulose template for copper nanoparticles as shown in the Table 5.1, the films were coded as PVA/TNFC-Cu0.6 and PVA/CMC-Cu0.6. Pure PVA, PVA/TNFC and PVA/CMC films were also prepared as control.

Table 4. 1 Weight percentage (wt.\%) of main element to the total weight of PVA, cellulose and copper.

\begin{tabular}{cccc}
\hline Sample Code & PVA (wt.\%) & Cellulose (wt.\%) & Copper (wt.\%) \\
\hline PVA/TNFC-Cu0.6 & 98.3 & 1.1 & 0.6 \\
PVA/CMC-Cu0.6 & 90.4 & 9.0 & 0.6 \\
PVA/TNFC & 98.9 & 1.1 & --- \\
PVA/CMC & 90.9 & 9.1 & --- \\
\hline
\end{tabular}




\subsubsection{Transmission electron microscopy (TEM)}

The morphology and particle size of copper nanoparticles on TNFC substrate were observed by JEOL TEM-2100 instrument (Tokyo, Japan) operating at $120 \mathrm{kV}$, the observation of CMC-copper nanoparticles were operated at $200 \mathrm{kV}$. TEM samples were typically prepared by dropping the hybrid TNFC-copper nanoparticles and CMC-copper nanoparticles solutions on a 200 mesh nickel grid coated with a carbon film.

\subsubsection{Ultraviolet-visible (UV-vis) characterization}

The resulting TNFC-copper nanoparticles solution and CMC-copper nanoparticles solution were optical characterized using Shimadzu UV-2550 spectrometer with a wavelength range of 200 - $900 \mathrm{~nm}$ (Kyoto, Japan). The bang gap energy of copper nanoparticles on cellulose templates was estimated using the equation (Eq. 1) (Dharma et al. 2009).

Band Gap Energy $(\mathrm{E})=\mathrm{hC} / \lambda \quad$ Eq. 1

Where $\mathrm{h}$ is planks constants, $\quad 6.626^{\times} 10^{-34} \mathrm{Js} ; \mathrm{C}$ is speed of light, $\quad 3.0^{\times} 10^{8} \mathrm{~m} / \mathrm{s} ; \lambda$ is cut off wavelength.

\subsubsection{X-ray diffraction (XRD) characterization}

The copper crystal structures within the final PVA/TNFC-Cu0.6 and PVA/CMC-Cu0.6 films were studied using a PANalytical X'Pert Pro X-ray diffractometer (Almelo, Netherlands) with a $\mathrm{Cu} \mathrm{K} \alpha \mathrm{X}$-ray source (X-ray wavelength: $0.15418 \mathrm{~nm}$ ). The X-ray source had a $40 \mathrm{kV}$ operating voltage, $45 \mathrm{~mA}$ current, $10-90^{\circ}$ angular $\left(2^{\theta}\right)$, and a step size of $0.0 \AA^{\ominus}(2)$. 


\subsubsection{X-ray photoelectron spectroscopy (XPS) characterization}

Oxidation states of copper on the surface of PVA/TNFC-Cu0.6 and PVA/CMC-Cu0.6 films were determined using a Physical Electronics VersaProbe 5000 X-ray Photoelectron Spectroscope with a monochromatic Aluminum K $\alpha$ X-ray source (Chanhassen, MI, USA). The base pressure in the high vacuum analysis chamber was around $2^{\times} 10^{-6} \mathrm{~Pa}$. An Aluminum X-ray source of $1486.6 \mathrm{eV}$ was used for photoelectron excitation with $\mathrm{X}$-ray power of $25 \mathrm{~W}$ and anode voltage of $15 \mathrm{kV}$. Pass energies of $117.4 \mathrm{eV}$ for survey scan and

$23.5 \mathrm{eV}$ for detail scan were used for the data acquisition with energy steps of $0.5 \mathrm{eV}$ for survey scan and $0.05 \mathrm{eV}$ for detail scan, respectively. PHI MultiPak software was used for element identification and peak fitting. The C1s peak at a binding energy of $284.8 \mathrm{eV}$ was used as the internal reference. A Shirley-type background was subtracted from the spectra and Gauss-Lorentz curves were used to fit the spectra.

\subsubsection{Antimicrobial activities of PVA/TNFC-Cu and PVA/CMC-Cu films}

E. coli lyfo-disks were reconstituted by crushing one pellet using a sterile spatula in $0.5 \mathrm{~mL}$ of sterile LTB. The content was aseptically transferred to $99.5 \mathrm{~mL}$ of sterile LTB and allowed to grow aerobically at $37^{\circ} \mathrm{C}$ for $24 \mathrm{~h}$ in an incubator/shaker set at $150 \mathrm{rpm}$ (C24, New Brunswick Scientific, New Jersey, USA). This procedure yielded $100 \mathrm{~mL}$ of E. coli stock culture. For experiments, a loopful of the stock culture was transferred to 100 $\mathrm{mL}$ of sterile LTB followed by incubation at $\quad 37^{\circ} \mathrm{C}$ for $24 \mathrm{~h}$ in the $\mathrm{C} 24$ incubator/shaker set at $150 \mathrm{rpm}$. This procedure yielded a culture with appropriately $10^{8}$ colony-forming units per milliliter (CFU/mL). A 2-mL aliquot of such culture was transferred to a surface of PVA/TNFC-Cu0.6 and PVA/CMC-Cu0.6 films and incubated at room temperature for 1 week. To prevent excessive evaporation of the E. coli culture from the surface of films, the films were kept in an aerobic environment with saturated humidity. After given exposure time, $1 \mathrm{~mL}$ of $E$. coli culture was removed from the surface of films to enumerate E. coli survivors. Prior to removal, the E. coli culture on films was 
carefully mixed to obtain an equal cell distribution. Enumeration was performed by a standard serial 10-fold dilution 
procedure and spread plating in a bio-safety cabinet under aseptic procedures (Black and Jaczynski 2006; Levanduski and Jaczynski 2008). A 1-mL aliquot of the E. coli culture removed from the surface of films was aseptically mixed with $9 \mathrm{~mL}$ of diluent (Butterfield phosphate buffer) followed by shaking the diluent bottle to uniformly distribute bacterial cells. Subsequent serial 10-fold dilutions were aseptically made by taking $10 \mathrm{~mL}$ of diluted sample and transferring it to a 90-mL diluent bottle. Survivors were enumerated on selective medium (Petrifilm ${ }^{\mathrm{TM}}$ E.coli/Coliform Count Plate) using a standard spread-plating technique. A 1.0-mL aliquot of each serial 10-fold dilution was pipetted and spread on $3 \mathrm{M}^{\mathrm{TM}}$ Petrifilm ${ }^{\mathrm{TM}}$ plates. The $3 \mathrm{M}^{\mathrm{TM}}$ Petrifilm ${ }^{\mathrm{TM}}$ plates were incubated at $35^{\circ} \mathrm{C}$ for $48 \mathrm{~h}$ (AOAC 1995, method 991.14).

Experiments were independently triplicated $(\mathrm{n}=3)$. Enumeration of E.coli Survivors in each equipment were performed in duplicated. Mean Values of E.coli survivors were used to calculate $\log$ reductions of E.coli on the tested films. All statistical analyses of data were performed using JMP version. 12 Statistical Software (Statistical Discovery. ${ }^{\text {TM }}$ From SAS).

\subsubsection{Inductively coupled plasma-optical emission (ICP-OES) spectrometry}

The copper amount leaching from PVA/TNFC-Cu0.6 and PVA/CMC-Cu0.6 films in the medium after given contact period was measured using Varian Vista-PRO CCD simultaneous ICP-OES (Palo Alto, USA). The specimens with the same dimensions of 28 $\mathrm{mm}^{\times} 28 \mathrm{~mm}$ and the same volume of $2 \mathrm{~mL}$ of the medium were used as did in antimicrobial activity testing that was presented previously. The same medium Lauryl tryptose broth (LTB) in the antimicrobial activity testing was also used for the measurement of copper ion release, but the LTB did not contain E.coli when it was used in copper ion release testing. The experiment was conducted in triplicate. 


\subsubsection{Thermo-gravimetric analyzer (TGA) characterization}

The thermal behaviors of pure PVA and its nanocomposite films were determined by TA Q50 thermo-gravimetric analyzer (New Castle, USA), with temperature ramp-up rate of $10{ }^{\circ} \mathrm{C} / \mathrm{min}$ while being purged with nitrogen at a flow rate of $20 \mathrm{~mL} / \mathrm{min}$. The sample weight was chosen between $3 \mathrm{mg}$ and $4 \mathrm{mg}$ for all of the samples tested.

\subsubsection{Dynamic mechanical analysis (DMA)}

Samples for DMA were prepared to dimension of $38 \mathrm{~mm}^{\times} 15 \mathrm{~mm} \times 0.35 \mathrm{~mm}$. Dynamic mechanical analysis was conducted in a tension-film mode on a TA Q800 DMA instrument (New Castle, USA), temperature scans were run from $30{ }^{\circ} \mathrm{C}$ to $150{ }^{\circ} \mathrm{C}$ at a heating rate of $2{ }^{\circ} \mathrm{C} / \mathrm{min}$ with a frequency of $1 \mathrm{~Hz}$ and amplitude $30 \mu_{\mathrm{m}}$.

\subsection{Results and discussion}

\subsubsection{Morphological characterization of the hybrid TNFC-copper nanoparticles and CMC-copper nanoparticles}

The transmission electron micrographs of the hybrid TNFC-copper nanoparticles and CMC-copper nanoparticles suspensions are presented in Figure 4.1. Copper nanoparticles are displayed as spherical shapes for both hybrid materials as shown in Figures 4.1a and 4.1b. The particle size of copper nanoparticles on TNFC template resulted of about $9.2 \pm$ $2.0 \mathrm{~nm}$ (Figure 4.1a), while for CMC template resulted of $12.5 \pm 2.8 \mathrm{~nm}$ (Figure 4.1b). Considering the standard deviation of these measurements, no statistical difference in the copper particle size was determined. 

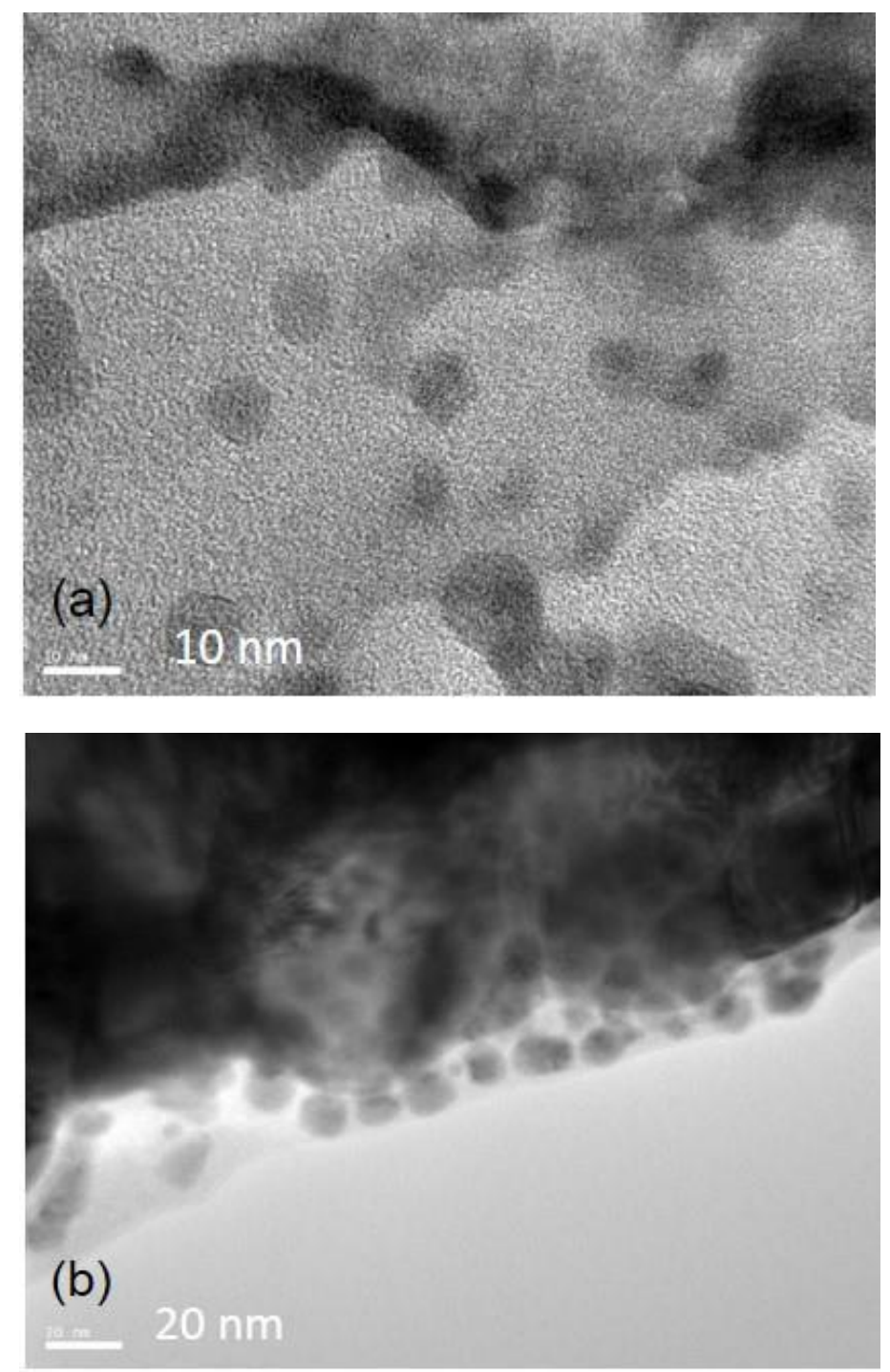

Figure 4. 1 TEM images of (a) TNFC-copper nanoparticles (scale bar = $10 \mathrm{~nm}$ ) and (b) CMC-copper nanoparticles (scale bar $=20 \mathrm{~nm}$ ).

\subsubsection{Ultraviolet-visible (UV-vis) analysis}

The UV-vis spectra show that the absorption band shoulder at about $250 \mathrm{~nm}$ in Figure 4.2 is caused by C6 aldehyde groups on the TEMPO nanofibrillated cellulose (Fukuzumi et al. 2009). The UV-vis spectra for both TNFC-copper nanoparticles and CMC-copper nanoparticles show the strong cut off wavelength $(\lambda)$ at around $525 \mathrm{~nm}$ when compared with pure TNFC and CMC as shown in Figure 4.2, and based on the equation for band gap 
energy $(E)=h C / \lambda$, the bandgap energy of copper nanoparticles on the both TNFC and CMC materials is estimated about $2.37 \mathrm{eV}$, indicating that $\mathrm{Cu}^{2+}$ on the cellulose templates has been reduced to $\mathrm{Cu}_{2} \mathrm{O}$ nanoparticles after chemical reduction by sodium borohydride. The bandgap energy for bulk $\mathrm{Cu}_{2} \mathrm{O}$ is $2.17 \mathrm{eV}$ (Yao et al. 2010), the reason for the bandgap energy of $\mathrm{Cu}_{2} \mathrm{O}$ nanoparticles being higher than bulk value of 2.17 is due to the quantum confinement effect with the decreasing particle size (Chang et al. 2005; Lu et al. 2005).

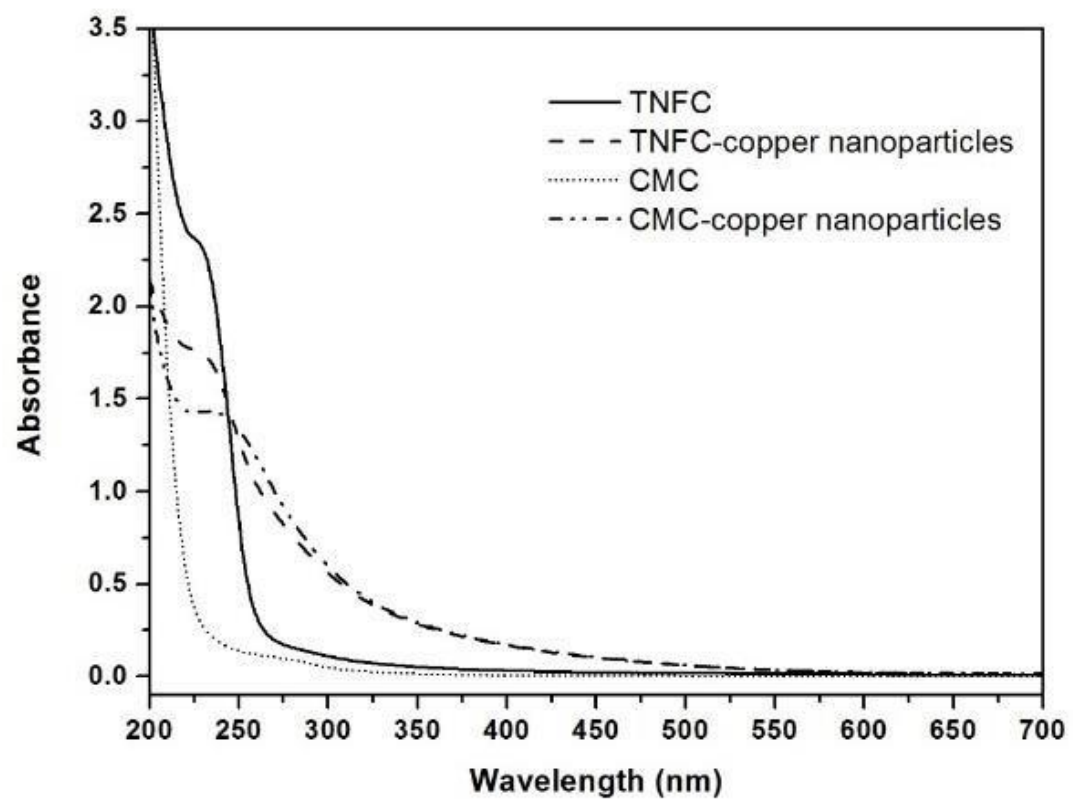

Figure 4. 2 UV-vis spectra of carboxylate cellulose suspensions and hybrid cellulosecopper nanoparticles suspensions.

\subsubsection{Copper crystal structure and oxidation states of copper within PVA/TNFC- Cu0.6 and PVA/CMC-Cu0.6 films}

Figure 4.3 presents the XRD patterns of PVA/TNFC-Cu0.6 and PVA/CMC-Cu0.6 composite materials matching the most prominent characteristic peaks of $\mathrm{Cu}_{2} \mathrm{O}[\mathrm{Cu}(\mathrm{I})]$ at

$2 \theta=36.5^{\circ}$ [International Center for Diffraction Data (ICDD): 01-078-2027]. In our previous work (Zhong et al. 2015b), we also demonstrated that CMC has favored more growth of crystalline $\mathrm{Cu}_{2} \mathrm{O}$ than TNFC. 
From the XRD analysis, the intensity of the characteristic peak for crystalline $\mathrm{Cu}_{2} \mathrm{O}[\mathrm{Cu}$ (I)] in PVA/CMC-Cu0.6 film is much stronger than that in PVA/TNFC-Cu0.6 film, indicating that higher amount of crystalline $\mathrm{Cu}_{2} \mathrm{O}$ is present in PVA/CMC-Cu0.6. $\mathrm{Cu}$ (I) ions possess higher and more efficient antimicrobial activities than $\mathrm{Cu}$ (II) (Meghana et al. 2015). $\mathrm{Cu}$ (I) ions can directly catalyze hydrogen peroxide and form ROS hydroxyl radical; hydrogen peroxide $\left(\mathrm{H}_{2} \mathrm{O}_{2}\right)$ is the byproducts during the aerobic respiration process of bacteria (Lemire et al. 2013). Cu (I)-catalyzed hydroxyl radical is the most powerful oxidizing radical that can react with almost every biological molecule (Buettner and Jurkiewicz 1996; Park et al. 2012). It may cause oxidative damage of protein, lipids, DNA or polysaccharides (Buettner and Jurkiewicz 1996; Lemire et al. 2013; Mathews et al. 2013; Palza 2015). Cu (II) ions also can participate in redox reaction and catalyze the formation of hydroxyl radicals from hydrogen peroxide through the Haber-Weiss reactions as shown in the following equations (Bremner 1988).

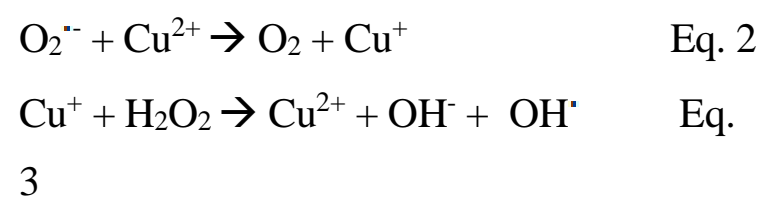

From Eq. $2 \mathrm{Cu}$ (II) ions are first reduced to $\mathrm{Cu}$ (I) ions by superoxide $\left(\mathrm{O}_{2}{ }^{{ }^{\mathrm{x}}}\right)$, which is another byproduct during aerobic respiratory process of bacteria; after that the produced $\mathrm{Cu}$

(I) ions are hydrogen peroxide catalyzed to generate hydroxyl radical (Eq. 3). It has been demonstrated by others that $\mathrm{Cu}$ (I) ions can be directly and more efficiently catalyzed to form hydroxyl radical; therefore $\mathrm{Cu}$ (I) ions have faster killing kinetic than $\mathrm{Cu}$ (II) ions. The generation of $\mathrm{Cu}$ (I)-catalyzed hydroxyl radical has been confirmed by other reports and $\mathrm{Cu}(\mathrm{I})$ appears to be the major species for inactivating E.coli and much more toxic than Cu (II) (Park et al. 2012; Shi et al. 2012; Meghana et al. 2015). In addition, it is reported that though under non-oxidative conditions the ability of $\mathrm{Cu}_{2} \mathrm{O}$ and $\mathrm{Cu}$ (I) salt also can directly and rapidly damages certain enzymes of metabolites, for example fumarase $\mathrm{A}$, in 
the case of E.coli. $\mathrm{CuO}$ salt [Cu (II)] also can produce damage on the enzyme fumarase A, but it is significantly slower than $\mathrm{Cu}_{2} \mathrm{O}$ salt [Cu (I)] (Meghana et al. 2015). 


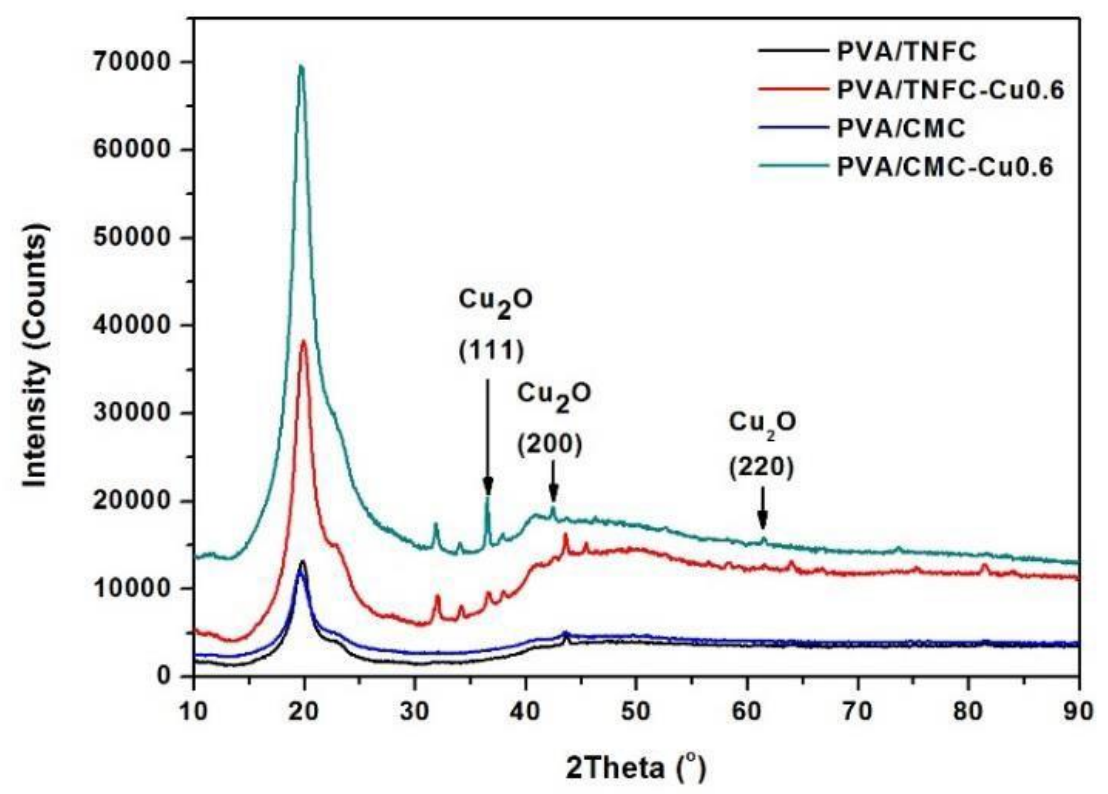

Figure 4. 3 XRD patterns of PVA composite films.

X-ray photoelectron spectroscopy was used to determine the oxidation states of copper on the topmost surface of the composite films. Figure 4.4a and 4.4b display the XPS spectra of PVA films containing copper nanoparticles. The two characteristics peaks $\mathrm{Cu} 2 \mathrm{p}_{1 / 2}$ and $\mathrm{Cu} 2 \mathrm{p}_{3 / 2}$ can be clearly observed in the expanded spectrum (inserts in Figures $4.4 \mathrm{a}$ and $4.4 \mathrm{~b}$ ), which confirm the presence of copper on the surface of both PVA composite films. In the inset of Figure $4.4 \mathrm{a}$, the $\mathrm{Cu} 2 \mathrm{p}_{3 / 2}$ peak centered at $933.7 \mathrm{eV}$ is consistent with $\mathrm{Cu}^{2+}$ (from $\mathrm{CuO}$ ) according to the report as shown in Table 4.2 (McIntyre and Cook 1975), which suggests that $\mathrm{Cu}^{2+}$ is present on the surface of PVA/TNFC-Cu0.6 film. The $\mathrm{Cu} 2 \mathrm{p}_{3 / 2}$ peak in the spectrum of PVA/CMC-Cu0.6 film is centered at $933.9 \mathrm{eV}$ as shown in the inset of Figure 4.4b, indicating that $\mathrm{Cu}^{2+}$ is also present on the surface of PVA/CMC-Cu0.6 film. UV-vis analysis discussed above suggested that $\mathrm{Cu}_{2} \mathrm{O}$ was formed on cellulosic templates. XPS analysis here indicated that $\mathrm{CuO}$ was found on both film surfaces when the 
hybrid cellulose- $\mathrm{Cu}_{2} \mathrm{O}$ nanoparticles were embedded in PVA matrix, which might be attributed to the oxidation of $\mathrm{Cu}_{2} \mathrm{O}$ to $\mathrm{CuO}$ on the film surface.
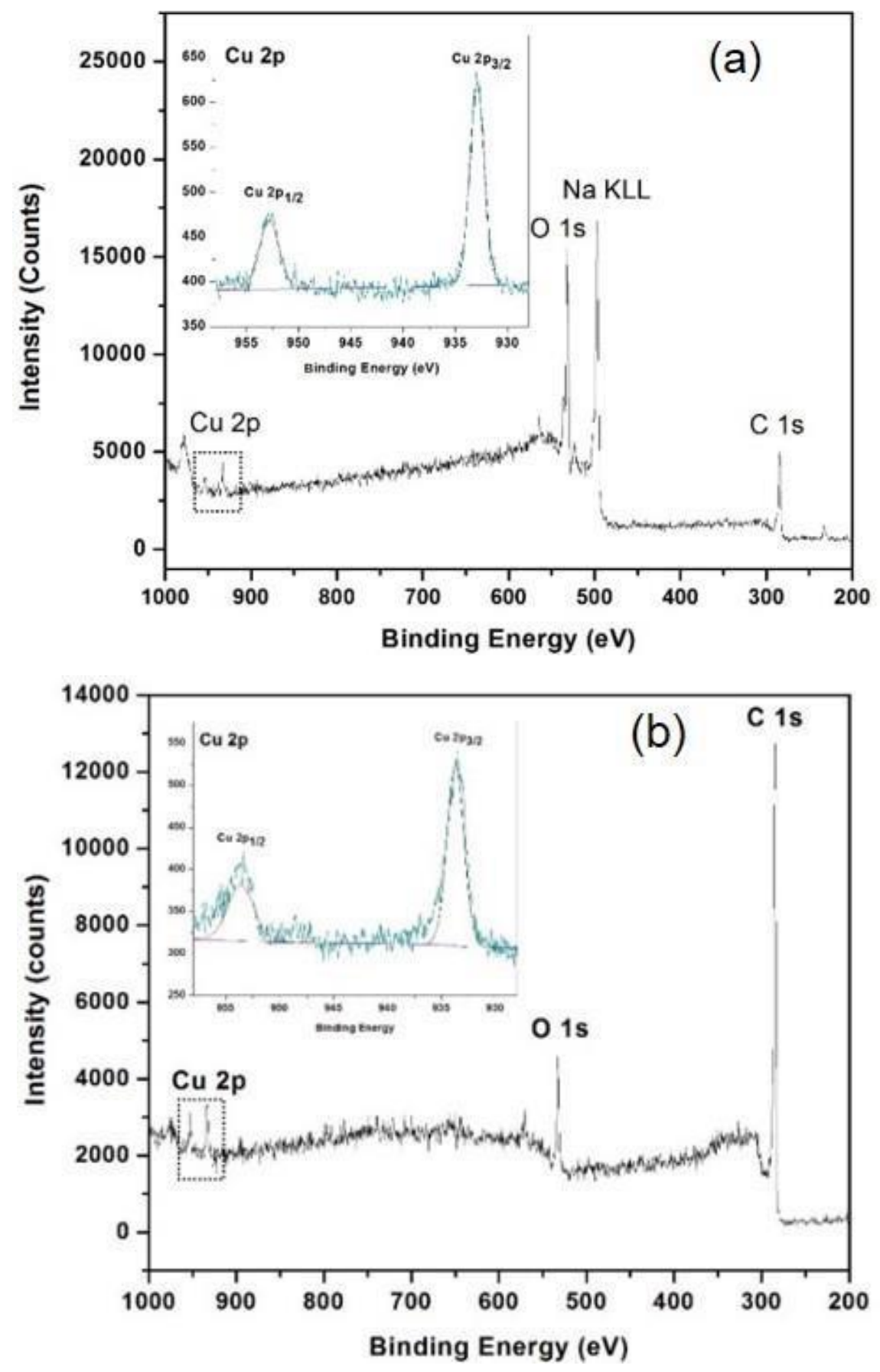

Figure 4. 4 XPS full survey scan of (a) PVA/TNFC-Cu0.6 and (b) PVA/CMC-Cu0.6. Inset: $\mathrm{Cu} 2 \mathrm{p}_{3 / 2}$ and $\mathrm{Cu} 2 \mathrm{p}_{1 / 2}$ XPS detail scan. 
Table 4. 2 Binding energy $\left(\mathrm{Cu} 2 \mathrm{p}_{3 / 2}\right)$ for copper different state of oxidation (McIntyre and Cook 1975).

\begin{tabular}{ccc}
\hline Chemical state & Formula & $\begin{array}{c}\text { Binding energy, } \mathrm{Cu} 2 \mathrm{p}_{3 / 2} \\
(\mathrm{eV})\end{array}$ \\
\hline $\mathrm{Cu}$ metal or $\left(\mathrm{Cu}^{0}\right)$ & $\mathrm{Cu}$ & $932.5 \pm 0.15$ \\
$\mathrm{Cu}(\mathrm{I})$ oxide or $\left(\mathrm{Cu}^{+}\right)$ & $\mathrm{Cu}_{2} \mathrm{O}$ & $932.5 \pm 0.2$ \\
$\mathrm{Cu}(\mathrm{II})$ oxide or $\left(\mathrm{Cu}^{2+}\right)$ & $\mathrm{CuO}$ & $933.8 \pm 0.2$ \\
$\mathrm{Cu}$ hydroxide or $\left(\mathrm{Cu}^{2+}\right)$ & $\mathrm{Cu}(\mathrm{OH})_{2}$ & $934.4 \pm 0.2$ \\
\hline
\end{tabular}

As a summary:

According to UV-vis (bulk analysis) copper state of oxidation in both hybrid cellulosecopper nanoparticles material was $\mathrm{Cu}(\mathrm{I})$.

Based on XRD (bulk analysis) copper state of oxidation in both films was $\mathrm{Cu}$ (I).

Based on XPS (topmost surface analysis) copper state of oxidation in both films was $\mathrm{Cu}$ (II).

\subsubsection{Antimicrobial activities of PVA/TNFC-Cu0.6 and PVA/CMC-Cu0.6 films}

The photographs presented in Figure 4.5 display representative examples of the results for bacterial enumeration of E.coli survivors on PVA/TNFC-Cu0.6 and PVA/CMC-Cu0.6 films after different exposure time. In general, the number of survival E.coli decreased with increasing exposure time. The survival E.coli population in PVA/CMC-Cu0.6 film is lower compared with PVA/TNFC-Cu0.6 films at the same exposure time. The enumeration of E.coli survivors (representative images shown in Figure 4.5) were log-converted and used to determine E.coli reductions as a function of exposure time (Figure 4.6). For both PVA composite films, the reduction of E.coli survivors occurred over time, but more slowly on PVA/TNFC films than on PVA/CMC films (See Figure 4.6). According to the data recorded and presented in Figure 4.6, there are statistical and significant differences in the antimicrobial performance of the films after their exposure to E.coli. After one-day exposure, there is a significant difference in the log microbial reduction $(P$ value $=0.033<$ 
0.05) of PVA/CMC-Cu0.6 when compared with PVA/TNFC-Cu0.6; the difference is even higher on the third day exposure $(p$ value $=0.0011<0.01)$. However, in term of the general antimicrobial performance, both PVA films were equivalently efficient since over $99.99 \%$ of E.coli were inactivated after 3-day exposure on either PVA/TNFC-Cu0.6 film or PVA/CMC-Cu0.6 film. It is important to mention, however, that approximately $90 \%$ less cellulosic material was required when using TNFC as template compared to CMC template to get similar films antimicrobial performance after 3-day exposure of E.coli (That is more than $4 \log$ microbial reduction - Figure 4.6).

The antimicrobial mechanism of PVA/copper nanoparticle nanocomposite against microorganisms has not been fully understood. Three of the most pertinent determinants of antimicrobial efficacy are: (1) metal nanoparticles, (2) metal ion release effectively and (3) metal ion-induced reactive oxygen species (ROS). A growing number of reports agrees that copper ion release is the driving force behind the antimicrobial properties of copper nanoparticles (Lemire et al. 2013; Mathews et al. 2013). Palza (2015) proposed that the antimicrobial mechanism of polymer composites containing metal is that water molecules coming from the food medium with dissolved oxygen diffused through the polymer matrix and reached the surface of embedded metal nanoparticles, and then triggered the dissolution or corrosion process, leaching metal ions to the composite film surface and eventually damaging cell membrane. In this study cellulose-copper nanoparticles were embedded in PVA matrix, we speculated that copper ions release from PVA matrix might play a critical role in inactivating E.coli.

As mentioned earlier, $\mathrm{Cu}_{2} \mathrm{O}[\mathrm{Cu}$ (I)] has demonstrated more effective antimicrobial performance than $\mathrm{Cu}$ (II). According to our $\mathrm{XRD}, \mathrm{Cu}$ (I) is highly available in the bulk of the films, and $\mathrm{Cu}$ (II) is available on the surface of the films (XPS analysis). We can infer then that there is a migration of ions not only from the surface of the films, but also from the bulk of them, generating generally good antimicrobial properties of the films. The higher amount of crystalline $\mathrm{Cu}_{2} \mathrm{O}[\mathrm{Cu}(\mathrm{I})]$ within PVA matrix might contribute to higher antimicrobial activity of PVA/CMC-Cu0.6 film compared to PVA/TNFC-0.6 film. 
PVA/TNFC-Cu0.6
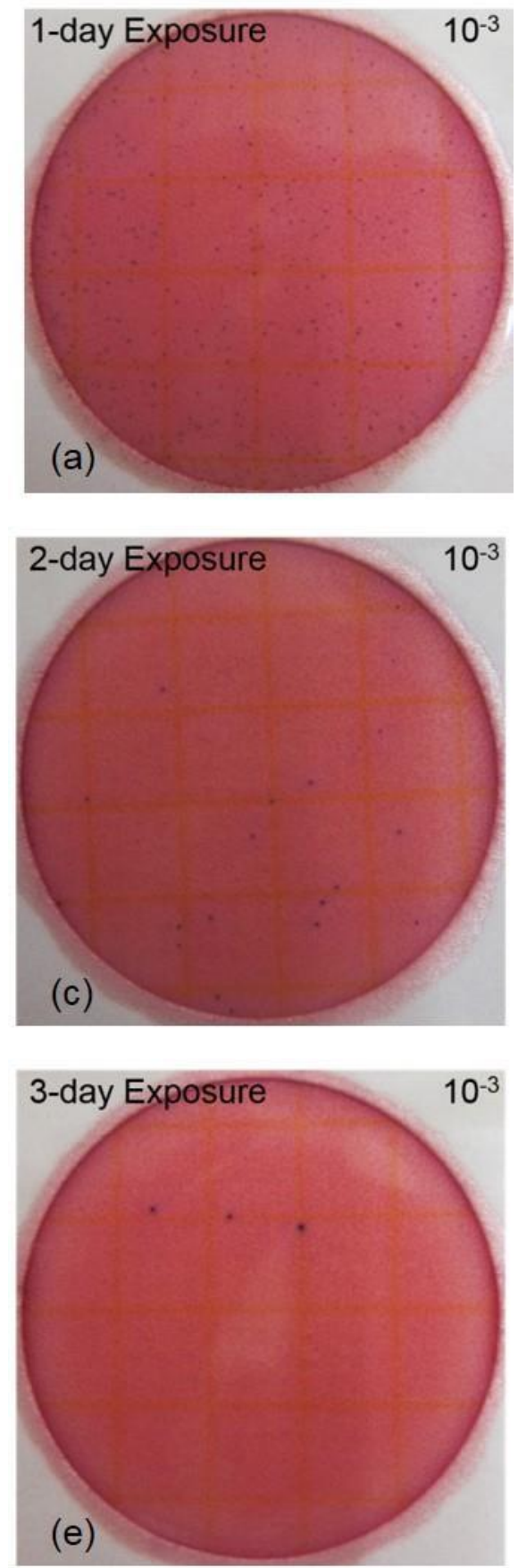

PVA/CMC-Cu0.6
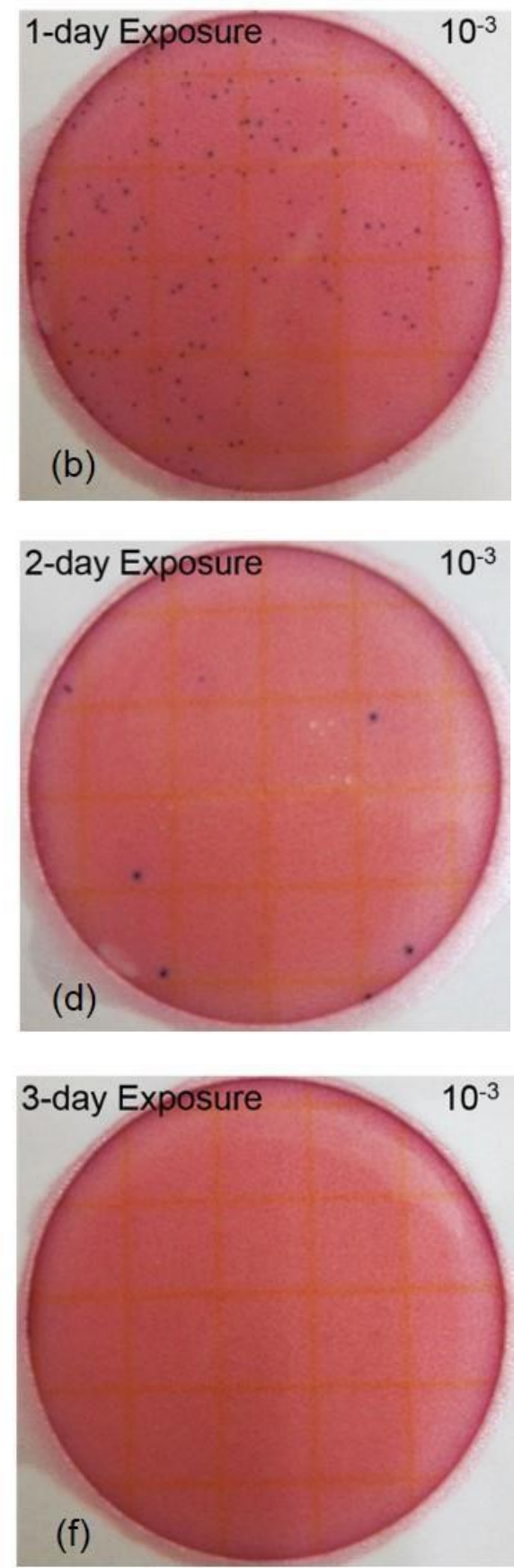

Figure 4. 5 Bacterial enumeration of the 24-h Escherichia coli culture exposed to PVA/TNFC-Cu0.6 film (a,c,e) and PVA/CMC-Cu0.6 film (b,d,f) after various exposure time. 


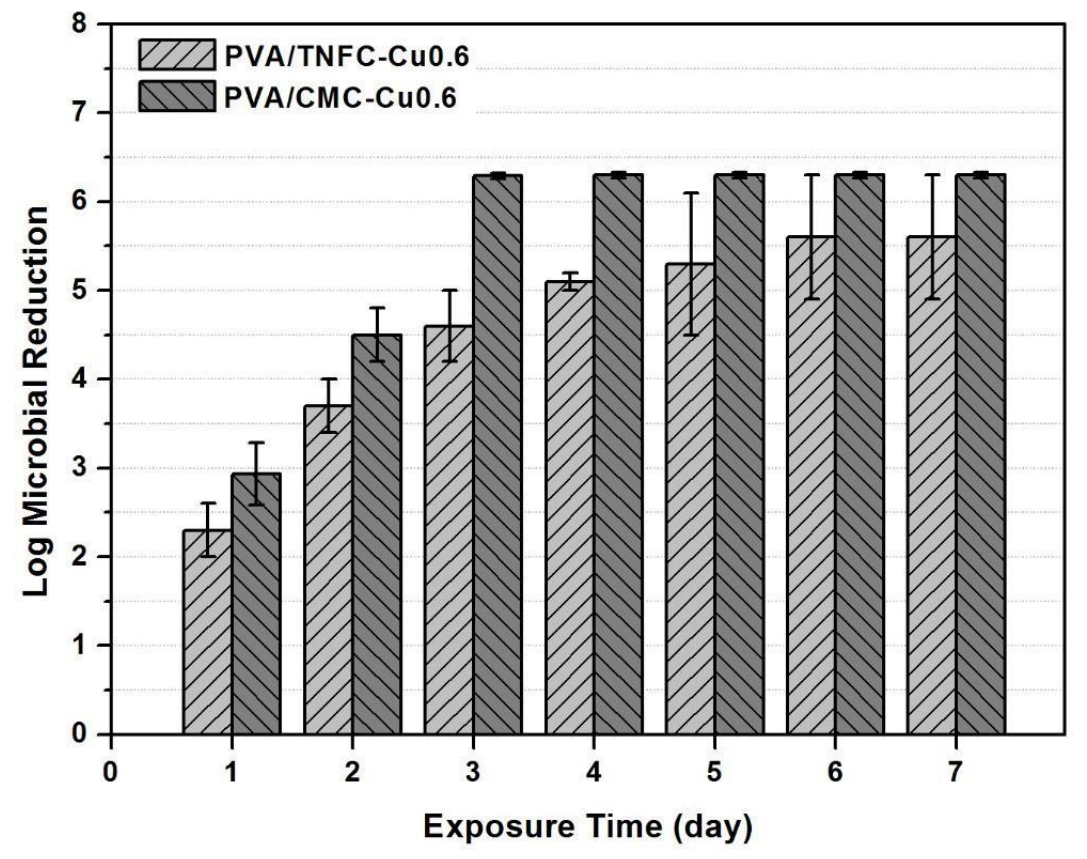

Figure 4. 6 Microbial reduction induced by PVA containing cellulose-copper nanoparticles after various exposure time.

\subsubsection{Copper ion release from PVA/cellulose-copper nanoparticles films}

Copper ion release from PVA/TNFC-Cu0.6 and PVA/CMC-Cu0.6 composite films was evaluated using Lauryl Tryptose Broth (LTB) as a release medium. As mentioned previously LTB was the food simulant to trigger the ion release from the films.

After the films immersion, copper concentration in the medium was determined by ICPOES each day for one week; the results are presented in Figure 4.7. From here, it is evident that the copper concentration increased with increasing contact time of composite films with the medium, and a high copper release rate was observed at the beginning of contact with the medium. Copper ion release rate from polymer/metal nanoparticles composite depends on the nature of the nanofillers and the polymer matrix (Radheshkumar and Münstedt, 2006). PVA is an hydrophilic polymer, and is highly sensitive to humidity. It is easy for water molecules with dissolved oxygen to reach the surface of copper 
nanoparticles embedded in PVA matrix, and to trigger dissolution, this may explain the high initial release of copper from both PVA composite films.

After 1-day of PVA/TNFC-Cu0.6 film exposure to the medium, copper concentration in the medium increased from 0 to $630.2 \pm 40.0 \mathrm{ppm}$ and up to $656.2 \pm 30.8 \mathrm{ppm}$ the second day; the change was not significant different between day 1 and day 2 exposure ( $p$ value $=0.42>0.05$ ). After a longer exposure time, the copper ion release continued slightly increasing but no significantly different respect the day before. Comparing day 1 and day 7 it is possible to appreciate a significant difference in the copper concentration on LTB ( $p$ value $=0.04<0.05$ ); for day 7 an increased up to $702.2 \pm 36.0 \mathrm{ppm}$ in copper concentration was observed. The corresponding film antimicrobial activity showed that 1-day exposure resulted in at least 2-log E.coli reduction while 7-day exposure resulted in at least 5-log reduction as shown in Figure 4.6. The difference in the antimicrobial activities is significant $(p$ value $=0.001<0.05$ ) and can be attributed to the increased amount of copper ions release with the increasing exposure time. A similar release behavior of copper ions from PVA/CMC-Cu0.6 films was also observed. That is, a high release rate was observed at the beginning of contact with the medium. The copper concentration in the medium increased from 0 to $553.0 \pm 17.0 \mathrm{ppm}$ after 1-day exposure, and then the copper concentration was slightly increasing with the increased exposure time without significant difference between consecutive days.

When compared the difference of copper concentration in the medium between films it is possible to notice that at the same exposure time, i.e. after 1-day contact with the medium, copper concentration in the medium is $630.2 \pm 40.0 \mathrm{ppm}$ and $553.0 \pm 17.0 \mathrm{ppm}$ for PVA/TNFC-Cu0.6 and PVA/CMC-Cu0.6, respectively; this difference was statistically significant $(p$ value $=0.037<0.05$ ). Sixty percent and $52.7 \%$ of copper were leaching from their corresponding films as shown in the inset of Figure 4.7 during the first day exposure. The release continued been statistically different between two PVA composites until day five. After the sixth day no significant difference $(p$ value $=0.88>0.05)$ in the medium copper concentration was found. The copper ions released faster from PVA/TNFC-Cu0.6 
films than from PVA/CMC-Cu0.6 films; this difference in release rate might be attributed to variation in the mass ratio of cellulosic template to copper in the PVA matrix. The mass ratio of the cellulosic material to copper in the PVA/CMC-Cu0.6 film was 15:1 and the mass ratio in PVA/TNFC-Cu0.6 film resulted of 1.8:1. Higher mass ratio is retarding the copper ion release rate due to the cellulosic network that is acting as a physical barrier to impede copper to release. However, the higher release rate of copper from PVA/TNFC was not directly related with its antimicrobial performance against E.coli $\mathrm{DH} 5 \alpha$. Both films demonstrated effective antimicrobial properties inactivating over $99.99 \%$ of E.coli DH5 $\alpha$ after 3 days of the bacteria exposure to the films.

There are several studies reporting the antimicrobial mechanism of copper. It has been postulated that the bacterial membrane is the site where copper exerts its bactericidal performance (Thurman et al. 1989; Lemire et al. 2013). Cationic copper ions, leaching from the films, can be attracted to negatively charged cell surface through electrostatic forces, and may undergo reactions at the surface to form organic complex with sulfur-, nitrogen- or oxygen-containing functional groups that is present in the membrane. The net negative charge on microorganisms is attributed to the presence of carboxyl, amino, guanidyl, and imidazole groups. These chemical groups, containing oxygen and nitrogen, act as ligands to coordinate metal ions. In addition, sulfhydryl groups in the bacterial membrane have a strong affinity for copper (Thurman et al. 1989). The presence of copper ions on cell membranes may result in protein dysfunction and the loss of enzyme activity and membrane function impair (Thurman et al. 1989; Raffi et al. 2010; Palza 2015). Likely, it has been previously reported that copper nanoparticles embedded in polymer resins led to the production of copper ions, which induced reactive oxygen species (ROS) that have been considered another main sources of copper toxicity (Hwang et al. 2008). The binding of copper ions to cell membranes can alter the permeability of cell membrane and damage its integrity, ultimately leading to cell death (Raffi et al. 2010; Wei et al. 2014). 


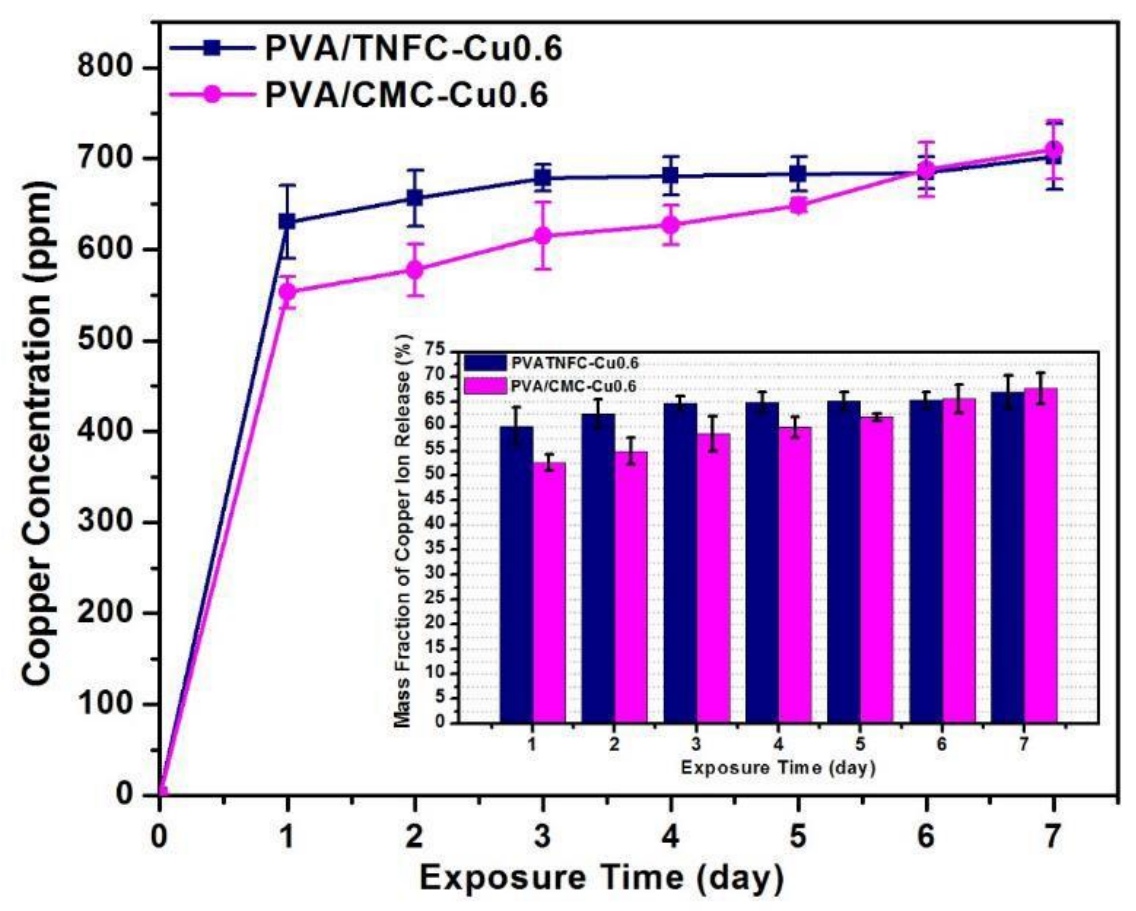

Figure 4. 7 The amount of copper leaching from PVA/TNFC-Cu0.6 and PVA/CMC$\mathrm{Cu} 0.6$ films and their corresponding mass fraction of copper ions leaching from the films (inset).

\subsubsection{Thermal analysis}

Figure 4.8a and 4.8b shows typical TGA and DTG curves of the PVA composite films. PVA/TNFC-Cu0.6 and PVA/CMC-Cu0.6 films display four distinct weight loss stages 1) loss of weakly physic-sorbed water at $30-210^{\circ} \mathrm{C} ; 2$ ) decomposition of cellulose-copper nanoparticles nanocomposites at $210-230{ }^{\circ} \mathrm{C}$; 3 ) decomposition of side chain of PVA at 230-380 ${ }^{\circ} \mathrm{C}$ and 4 ) decomposition of main chain of PVA at $380-550{ }^{\circ} \mathrm{C}$. 

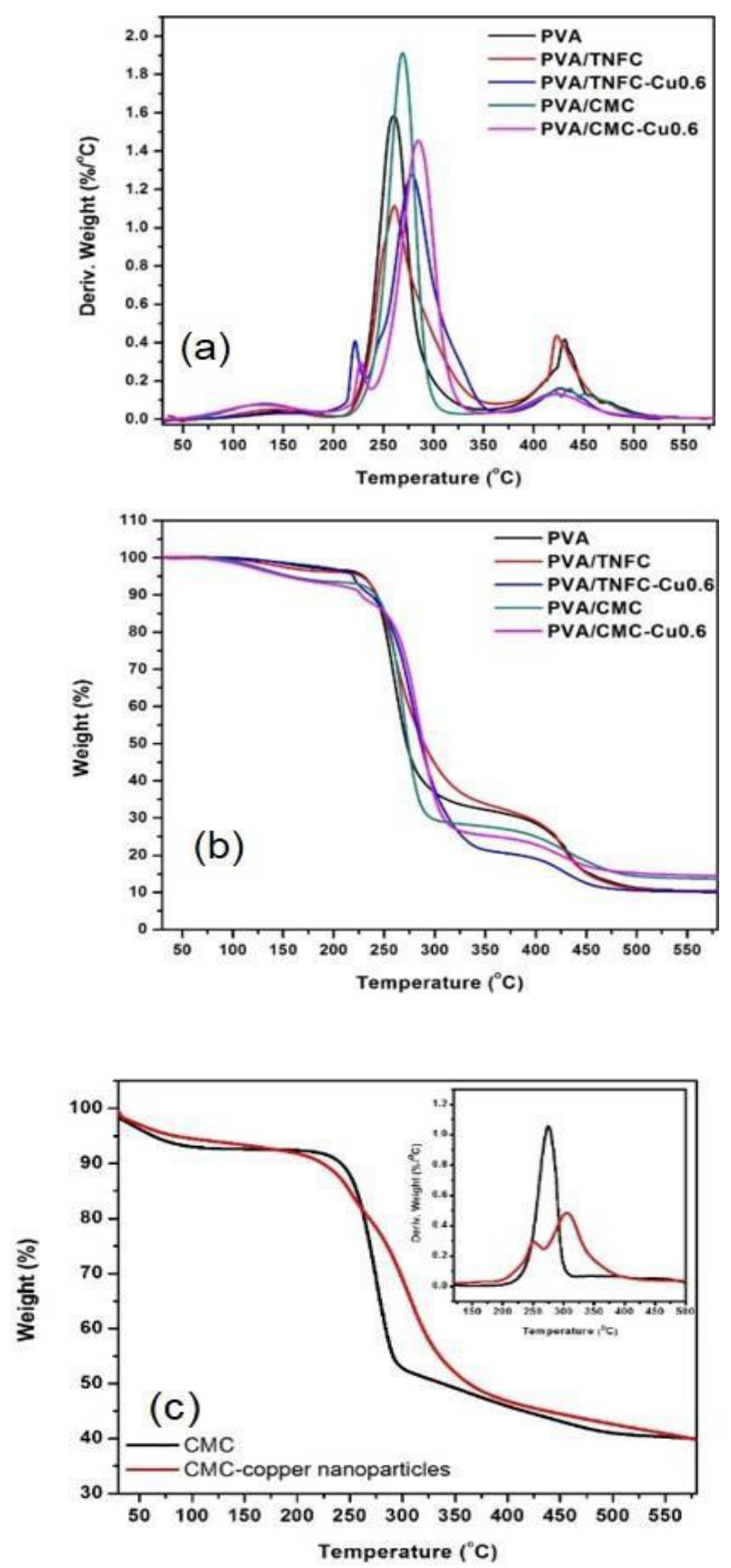

Figure 4.8 (a) TGA curves for the pure PVA, PVA/TNFC film, and PVA/TNFC-Cu0.6 film, PVA/CMC film, and PVA/CMC-Cu0.6 film; (b) the corresponding DTG curves (Peak temperature $\left(T_{\max }\right)$ of PVA, PVA/TNFC, PVA/TNFC-Cu0.6, PVA/CMC, PVA/CMC-Cu0.6 was $260,263,278,269$, and $285^{\circ} \mathrm{C}$, respectively); (c) TGA cure for CMC and CMC-copper nanoparticles and its corresponding DTG cure (Inset). 
The weight loss around $225^{\circ} \mathrm{C}$ for both PVA/TNFC-Cu0.6 and PVA/CMC-Cu0.6 films was attributed to the decomposition of the hybrid cellulose-copper nanoparticles nanocomposite within the PVA matrix. The phenomenon is consistent with the thermal behavior of CMC-copper nanoparticles alone as shown in Figure 4.8c. Major weight losses were observed in the range of $210-550{ }^{\circ} \mathrm{C}$, which corresponded to the structural decomposition of PVA and thermal degradation of cellulose. $T_{\max }$ is the decomposition temperature corresponding to the maximum weight loss and relates to the maximum decomposition rate. In Figure $4.8 \mathrm{~b}$, we can see that $T_{\max }$ of PVA/TNFC-Cu0.6 and PVA/CMC-Cu0.6 films shifted to higher temperature compared to that of pure PVA, an increase of $T_{\max }$ was observed from $260{ }^{\circ} \mathrm{C}$ to $278{ }^{\circ} \mathrm{C}$ for PVA/TNFC-Cu0.6 composite film. The $T_{\max }$ of PVA composite was brought up to $285^{\circ} \mathrm{C}$ after CMC-copper nanoparticles were incorporated, compared to pure PVA. A higher amount of crystalline $\mathrm{Cu}_{2} \mathrm{O}$ within PVA/CMC-Cu0.6 might contribute to higher $T_{\max }$. This explains why PVA/CMC-Cu0.6 has higher $T_{\max }$ than PVA/TNFC-Cu0.6. The major degradation peak when shifting to higher temperature is an indicator of enhanced thermal stability ( $\mathrm{Lu}$ et al. 2008). The thermal decomposition of PVA/TNFC-Cu0.6 and PVA/CMC-Cu0.6 films shifted slightly toward high temperature, suggesting that the composite films had higher thermal stability that might be as a consequence of the presence of crystalline structure of cellulose and copper nanoparticles embedded in the PVA matrix.

\subsubsection{Dynamic mechanical analysis}

Dynamic mechanical analysis measures stiffness and damping of materials; these characteristics are reported respectively as storage modulus $\left(E^{\prime}\right)$ and $\tan ^{\delta}$. Figures 4.9a) and 4.9b) display the temperature dependence of storage modulus (E') and $\tan ^{\delta}$ for PVA and various PVA composite films. All PVA composite films have higher storage modulus than pure PVA as shown in Figure 4.9a. At the PVA glass transition temperature $\left(T_{g}=48^{\circ} \mathrm{C}\right)$ the correspondent storage modulus from higher to lower resulted as: PVA/CMC-Cu0.6

$>\mathrm{PVA} / \mathrm{TNFC}-\mathrm{Cu} 0.6 \approx \mathrm{PVA} / \mathrm{TNFC}>\mathrm{PVA} / \mathrm{CMC}>\mathrm{PVA}$; the storage modulus increased 
by $217 \%, 164 \%, 158 \%$ and $33 \%$ respectively, when compared with the storage modulus of 
pure PVA. The incorporation of copper nanoparticles on CMC raw material significantly increased the composites' storage modulus; however, not much increase is observed when TNFC is used as template. This fact might be attributed to the morphology of the cellulosic materials and their concentration on the films. It is important to mention here that the CMC loading in the PVA/CMC film was 9.0 wt.\%, whereas TNFC loading was $1.1 \mathrm{wt} \%$. With higher cellulose loading in the matrix, the possible increment of aggregation led to poor cellulose/matrix interfacial interaction, and finally might reduce the modulus of the composite film. The addition of copper nanoparticles on CMC helped reduce the cellulose aggregations due to electrostatic repulsion (Zhong et al. 2015b) and therefore increased the composites' storage modulus. The effect is not that dramatic when TNFC is used since less cellulosic material is used.

The significant reinforcing effects of metallic nanoparticles on the dynamic mechanical properties have been reported previously by others (Chou et al. 2006; Gautam and Ram 2010). Chou et al. reported that the incorporation of $3.02^{\times} 10^{-3}$ wt.\% silver nanoparticles in polyurethane (PU) resulted in the increase in the storage modulus of its composite up to $117.4 \%$ at $37^{\circ} \mathrm{C}$. Gautam and Ram reported that the storage modulus of polyvinyl alcohol composites increased up to $22.2 \%$ at $-25{ }^{\circ} \mathrm{C}$ after the addition of 0.2 wt.\% silver nanoparticles; silver nanoparticles can restrict the mobility of the polymer chain due to their large surface area and their van der Waals attraction to the polymer matrix, which causes the strengthening of mechanical properties of PVA-silver nanocomposite films.

The other factor that also might have contributed to the increase in the storage modulus for PVA/CMC-Cu0.6 films was the copper crystallinity (Chou et al. 2006; Gautam and Ram, 2010). From the XRD analysis in our previous work, the intensity of the characteristic peak for crystalline $\mathrm{Cu}_{2} \mathrm{O}(\mathrm{Cu}(\mathrm{I}))$ in $\mathrm{PVA} / \mathrm{CMC}-\mathrm{Cu} 0.6$ film resulted significant much stronger than that in PVA/TNFC-Cu0.6 film, indicating that higher amount of crystalline $\mathrm{Cu}_{2} \mathrm{O}$ is present in PVA/CMC-Cu0.6. 

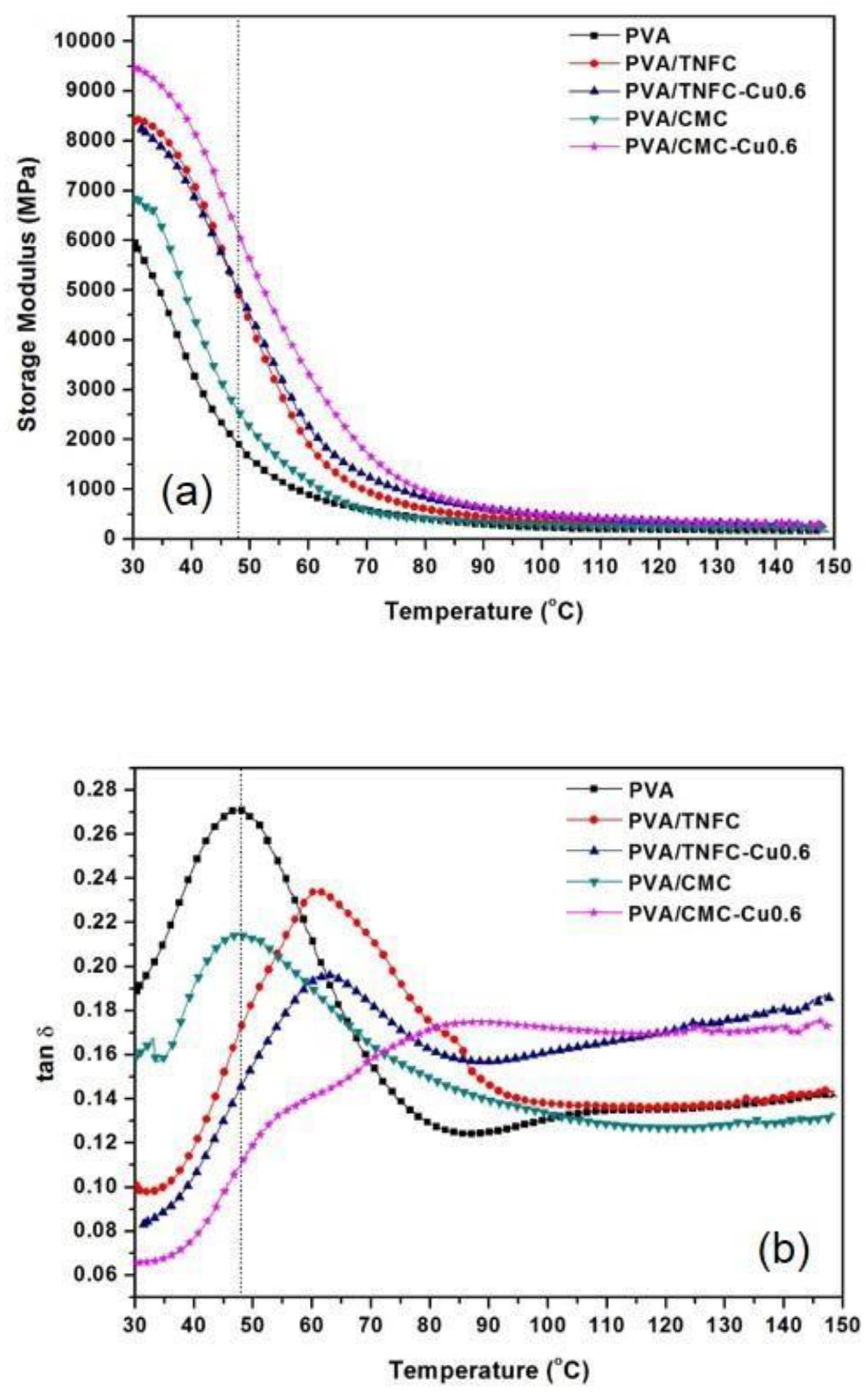

Figure 4. 9 (a) Storage modulus as a function of temperature for PVA composite films and (b) corresponding $\tan \delta$.

The loss factor $\tan \delta$ is a measure of damping and an indicator of how efficiently the materials lose energy to molecular rearrangement and internal friction; the peak in $\tan \delta$ 
corresponds to the glass transition temperature $(T g$ ) (Shaffer and Windle 1999; Menard 2008). From Figure $4.9 \mathrm{~b}$ we can see the intensity of $\tan ^{\delta}$ is reduced due to the reducing friction of polymer matrix and good adhesion between cellulose and the matrix (Shaffer and Windle, 1999; Amash and Zugenmaier 2000; Lu et al. 2008). The presence of TNFC and TNFC-copper nanoparticles significantly affected the peak position of PVA/TNFC and PVA/TNFC-Cu0.6 films, indicating that the incorporation of TNFC and TNFC-copper nanoparticles has enhanced their glass transition temperature. Several reports have attributed this to the formation of strong percolated interface of TNFC and matrix at low TNFC loading ( $\mathrm{Lu}$ et al. 2008; Ramanathan et al. 2008; Zaragoza-Contreras et al. 2009). The $\tan ^{\delta}$ peak for PVA/CMC has slightly shifted to low temperature probably due to cellulose aggregation and bad cellulose/matrix interaction, which was led by the overloading cellulose (e.g., 9 wt.\%) in the matrix. It is interesting to note that the $\tan { }^{\delta}$ peak for PVA/CMC-Cu0.6 became broader. It is too flat and diffuse to see the exact position for $T_{g}$. It seems that the $\tan ^{\delta}$ peak shifted to higher temperature. The shifting of $T_{g}$ to higher temperature might be associated with the decreased mobility of the chains by the incorporation of crystal $\mathrm{Cu}_{2} \mathrm{O}$. Nano-size crystalline $\mathrm{Cu}_{2} \mathrm{O}$ can restrict the mobility of polymer chains because of their high surface areas and their good interfacial interaction between the nanoparticles and the polymer matrix (Gautam and Ram 2010).

\subsection{Conclusions}

- Approximately 90\% less cellulosic material was required when using TNFC as copper template compared to $\mathrm{CMC}$ template to get similar films antimicrobial performance.

- Both PVA composite films demonstrated strong antimicrobial activities against E.coli DH 5 $\alpha$; over $99.99 \%$ of E.coli were inactivated after 3-day exposure of E.coli to either PVA/TNFC-Cu0.6 film or PVA/CMC-Cu0.6 film.

- $\mathrm{Cu}$ (I) was available in the bulk of both, PVA-CMC films and PVA-TNFC films. 
- $\mathrm{Cu}$ (II) was only available in the surface of both, PVA-CMC films and PVA-TNFC films.

- Copper ion release from PVA/TNFC films is significantly higher compared to the release from PVA/CMC after one week exposure. However, both films were equally efficient to inactivate over $99.99 \%$ of E.coli $\mathrm{DH} 5 \alpha$ after 3 days of bacterial exposure to the films.

- The incorporation of TNFC-copper nanoparticles and CMC-copper nanoparticles on the PVA matrix increased the maximum decomposition temperature by $18{ }^{\circ} \mathrm{C}$ and $25^{\circ} \mathrm{C}$ compared to pure PVA, respectively.

- The storage modulus of the PVA composite films at $48{ }^{\circ} \mathrm{C}$ increased up to $164 \%$ and $217 \%$ with the incorporation of TNFC-copper nanoparticles and CMC-copper nanoparticles, respectively.

\subsection{References}

Amash, A., \& Zugenmaier, P. (2000). Morphology and properties of isotropic and oriented samples of cellulose fibre-polypropylene composites. Polymer, 41(4), 1589-1596.

AOAC (1995). Official methods of analysis. 16th ed. Association of Official Analytical Chemists International, Gaithersburg, MD.

Black, J. L., \& Jaczynski, J. (2006). Temperature effect on inactivation kinetics of Escherichia coli O157: H7 by electron beam in ground beef, chicken breast meat, and trout fillets. Journal of Food Science, 71(6), M221-M227.

Borkow, G., \& Gabbay, J. (2009). Copper, an ancient remedy returning to fight microbial, fungal and viral infections. Current Chemical Biology, 3(3), 272-278. 
Bremner, I. (1998). Manifestations of copper excess. The American Journal of Clinical Nutrition, 67(5), 1069S-1073S.

Buettner, G. R., \& Jurkiewicz, B. A. (1996). Catalytic metals, ascorbate and free radicals: combinations to avoid. Radiation Research, 145(5), 532-541.

Cady, N. C., Behnke, J. L.. \& Strickland, A. D. (2011). Copper-Based Nanostructured Coatings on Natural Cellulose: Nanocomposites Exhibiting Rapid and Efficient

Inhibition of a Multi-Drug Resistant Wound Pathogen, A. baumannii, and Mammalian Cell Biocompatibility In Vitro. Advanced Functional Materials, 21(13), 2506-2514.

Chakraborty, A., Sain, M., \& Kortschot, M. (2006). Reinforcing potential of wood pulpderived microfibres in a PVA matrix. Holzforschung, 60(1), 53-58.

Chang, Y., Teo, J. J., \& Zeng, H. C. (2005). Formation of colloidal CuO nanocrystallites and their spherical aggregation and reductive transformation to hollow $\mathrm{Cu}_{2} \mathrm{O}$ nanospheres. Langmuir, 21(3), 1074-1079.

Cheng, Q., Wang, S., Rials, T. G., \& Lee, S. H. (2007). Physical and mechanical properties of polyvinyl alcohol and polypropylene composite materials reinforced with fibril aggregates isolated from regenerated cellulose fibers. Cellulose, 14(6), 593-602.

Chou, C. W., Hsu, S. H., Chang, H., Tseng, S. M., \& Lin, H. R. (2006). Enhanced thermal and mechanical properties and biostability of polyurethane containing silver nanoparticles. Polymer Degradation and Stability, 91(5), 1017-1024.

Dharma, J., Pisal, A., \& Shelton, C. T. (2009). Simple method of measuring the band gap energy value of $\mathrm{TiO} 2$ in the powder form using a UV/Vis/NIR spectrometer. Application Note Shelton, CT: PerkinElmer. 
Fukuzumi, H., Saito, T., Iwata, T., Kumamoto, Y., \& Isogai, A. (2009). Transparent and high gas barrier films of cellulose nanofibers prepared by TEMPO-mediated oxidation. Biomacromolecules, 10, 162-165.

Gautam, A., \& Ram, S. (2010). Preparation and thermomechanical properties of Ag-PVA nanocomposite films. Materials Chemistry and Physics, 119, 266-271.

Gong, P., Li, H., He, X., Wang, K., Hu, J., Tan, W., Zhang, S., \& Yang, X. (2007). Preparation and antibacterial activity of $\mathrm{Fe}_{3} \mathrm{O}_{4} @ \mathrm{Ag}$ nanoparticles. Nanotechnology, 18(28), 285604.

He, J., Kunitake, T., \& Nakao, A. (2003). Facile in situ synthesis of noble metal nanoparticles in porous cellulose fibers. Chemistry of Materials, 15(23), 4401-4406.

Hostynek, J. J., \& Maibach, H. I. (2004). Copper hypersensitivity: dermatologic aspects*. Dermatologic Therapy, 17(4), 328-333.

Hwang, E. T., Lee, J. H., Chae, Y. J., Kim, Y. S., Kim, B. C., Sang, B. I., \& Gu, M. B. (2008). Analysis of the Toxic Mode of Action of Silver Nanoparticles Using

Stress-Specific Bioluminescent Bacteria. Small, 4(6), 746-750.

Isogai, A., Saito, T., \& Fukuzumi, H. (2011). TEMPO-oxidized cellulose nanofibers. Nanoscale, 3(1), 71-85.

Jia, B., Mei, Y., Cheng, L., Zhou, J., \& Zhang, L. (2012). Preparation of copper nanoparticles coated cellulose films with antibacterial properties through one-step reduction. ACS Applied Materials \& Interfaces, 4(6), 2897-2902. 
Lemire, J. A., Harrison, J. J., \& Turner, R. J. (2013). Antimicrobial activity of metals: mechanisms, molecular targets and applications. Nature Reviews Microbiology, 11, 371-384.

Levanduski, L., \& Jaczynski, J. (2008). Increased resistance of Escherichia coli O157: H7 to electron beam following repetitive irradiation at sub-lethal doses. International Journal of Food Microbiology, 121(3), 328-334.

Liu, D., Sun, X., Tian, H., Maiti, S., \& Ma, Z. (2013). Effects of cellulose nanofibrils on the structure and properties on PVA nanocomposites. Cellulose, 20(6), 2981-2989.

Llorens, A., Lloret, E., Picouet, P. A., Trbojevich, R., \& Fernandez, A. (2012). Metallicbased micro and nanocomposites in food contact materials and active food packaging. Trends in Food Science \& Technology, 24(1), 19-29.

Lu, C., Qi, L., Yang, J., Wang, X., Zhang, D., Xie, J., \& Ma, J. (2005). One-Pot Synthesis of 'Octahedral ${ }^{\mathrm{Cu}}{ }_{2} \mathrm{O}$ Nanocages via a Catalytic Solution Route. Advanced Materials, 17(21), 2562-2567.

Lu, J., Wang, T., \& Drzal, L. T. (2008). Preparation and properties of microfibrillated cellulose polyvinyl alcohol composite materials. Composites Part A: Applied Science and Manufacturing, 39(5), 738-746.

Mathews, S., Hans, M., Mücklich, F., \& Solioz, M. (2013). Contact killing of bacteria on copper is suppressed if bacterial-metal contact is prevented and is induced on iron by copper ions. Applied and Environmental Microbiology, 79(8), 2605-2611.

McIntyre, N. S., \& Cook, M. G. (1975). X-ray photoelectron studies on some oxides and hydroxides of cobalt, nickel, and copper. Analytical Chemistry, 47(13), 2208-2213. 
Meghana, S., Kabra, P., Chakraborty, S., \& Padmavathy, N. (2015). Understanding the pathway of antibacterial activity of copper oxide nanoparticles. RSC Advances, 5, 12293-12299.

Menard, K. P. (2008). Dynamic mechanical analysis: a practical introduction. CRC press.

Moon, R. J., Martini, A., Nairn, J., Simonsen, J., \& Youngblood, J. (2011). Cellulose nanomaterials review: structure, properties and nanocomposites. Chemical Society Reviews, 40(7), 3941-3994.

Padalkar, S., Capadona, J. R., Rowan, S. J., Weder, C., Won, Y. H., Stanciu, L. A., \& Moon, R. J. (2010). Natural biopolymers: novel templates for the synthesis of nanostructures. Langmuir, 26(11), 8497-8502.

Palza, H. (2015). Antimicrobial Polymers with Metal Nanoparticles. International Journal of Molecular Sciences, 16, 2099-2116.

Park, H. J., Nguyen, T. T., Yoon, J., \& Lee, C. (2012). Role of reactive oxygen species in Escherichia coli inactivation by cupric ion. Environmental Science \& Technology, 46(20), 11299-11304.

Radheshkumar, C., \& Münstedt, H. (2006). Antimicrobial polymers from polypropylene/silver composites- $\mathrm{Ag}+$ release measured by anode stripping voltammetry. Reactive and Functional Polymers, 66(7), 780-788.

Raffi, M., Mehrwan, S., Bhatti, T. M., Akhter, J. I., Hameed, A., Yawar, W., \& ul Hasan, M. M. (2010). Investigations into the antibacterial behavior of copper nanoparticles against Escherichia coli. Annals of Microbiology, 60(1), 75-80. 
Ramanathan, T., Abdala, A. A., Stankovich, S., Dikin, D. A., Herrera-Alonso, M., Piner, R. D., Adamson, D. H., Schniepp, H. C., Chen, X., Ruoff, R. S., Nauyen, S. T., Aksay, I. A., Prud'homme, R. K., \& Brinson, L. C. (2008). Functionalized graphene sheets for polymer nanocomposites. Nature Nanotechnology, 3(6), 327-331.

Saito, T., \& Isogai, A. (2004). TEMPO-mediated oxidation of native cellulose. The effect of oxidation conditions on chemical and crystal structures of the water-insoluble fractions. Biomacromolecules, 5(5), 1983-1989.

Shaffer, M. S. P., \& Windle, A. H. (1999). Fabrication and characterization of carbon nanotube/poly (vinyl alcohol) composites. Advanced Materials, 11(11), 937-941.

Shi, M., Kwon, H. S., Peng, Z., Elder, A., \& Yang, H. (2012). Effects of surface chemistry on the generation of reactive oxygen species by copper nanoparticles. Acs Nano, 6(3), 2157-2164.

Thurman, R. B., Gerba, C. P., \& Bitton, G. (1989). The molecular mechanisms of copper and silver ion disinfection of bacteria and viruses. Critical Reviews in Environmental Science and Technology, 18(4), 295-315.

Wei, X., Yang, Z., Wang, Y., Tay, S. L., \& Gao, W. (2014). Polymer antimicrobial coatings with embedded fine $\mathrm{Cu}$ and $\mathrm{Cu}$ salt particles. Applied Microbiology and Biotechnology, 98(14), 6265-6274.

Yao, K. X., Yin, X. M., Wang, T. H., \& Zeng, H. C. (2010). Synthesis, self-assembly, disassembly, and reassembly of two types of $\mathrm{Cu}_{2} \mathrm{O}$ nanocrystals unifaceted with $\{001\}$ or $\{110\}$ planes. Journal of the American Chemical Society, 132(17), 6131-6144.

Zaragoza-Contreras, E., Hernandez-Escobar, C. A., Mendoza-Duarte, M. E., FloresGallardo, S. G., Ibarra-Gomez, R., \& Marquez-Lucero, A. (2009). Thermal and 
mechanical analysis of silver/carbon nanoparticle--PMMA nanocomposites obtained by miniemulsion polymerization. Polymer Journal, 41(10), 816-821.

Zhong, T., Oporto, G. S., Jaczynski, J., Tesfai, A. T., \& Armstrong, J. (2013). Antimicrobial properties of the hybrid copper nanoparticles-carboxymethyl cellulose. Wood and Fiber Science, 45(2), 215-222.

Zhong, T., Oporto, G. S., Jaczynski, J., \& Jiang, C. (2015a). Nanofibrillated Cellulose and Copper Nanoparticles Embedded in Polyvinyl Alcohol Films for Antimicrobial Applications. BioMed Research International, 2015, 1-8.

Zhong, T., Oporto, G. S., Peng, Y., Xie, X., \& Gardner, D. J. (2015b) Drying cellulosebased materials containing copper nanoparticles. Cellulose, 22, 2665-2681. 
CHAPTER 5: MICROSTRUCTURE, MECHANICAL, THERMAL AND ANTIMICROBIAL PROPERTIES OF FREEZE-DRIED
HYBRID COPPER NANOPARTICLES AND CELLULOSE BASED
MATERIALS EMBEDDED IN THERMOPLASTIC RESINS

\subsection{Chapter abstract}

Copper nanoparticles were synthesized "in situ" on carboxymethyl cellulose (CMC) and TEMPO-oxidized nanofibrillated cellulose (TNFC). These hybrid materials were incorporated in thermoplastic resins such as polypropylene (PP) and polylactic acid (PLA). Composites were prepared using melting extrusion method on a twin-screw extruder equipped with both injection molding process and a dry film formation process. The morphological observation of composites revealed that some of the hybrid material well distributed in the thermoplastic resins, however a large amount of the hybrid material remained as aggregates. The tensile strengths of PP and PLA composites decreased by 19.2\% after the incorporation of the hybrid material when compared to the pure thermoplastic resins. Thermal analysis showed no distinctive changes in the glass transition temperature and melting temperature for both PP and PLA composites after the incorporation of the hybrid material. The incorporation of hybrid materials significantly increased the crystallinity of PP and PLA composites compared with the pure thermoplastic resins was observed. The amount of copper ions released from the film composites was determined using atomic absorption spectrometry. The preliminary results showed that even though copper ions migrating from the surface of films were detected, the amount of leaching ions is low and the migration rate is insufficient to inactivate E.coli DH5 $\alpha$. No migration of copper ions was determined for those extruded-injected composites. 


\subsection{Introduction}

The feasibility to incorporate antimicrobial materials as active nanocomponents into thermoplastic resins has offered new pathways to develop novel application in areas such as packaging, medicine, safety, etc. (Zhang et al. 2007; Duncan 2011). However, little work has been performed with respective to the capability of releasing the active materials and how the incorporation of the active materials would affect the mechanical properties of the final composites (Fernandez et al. 2010; Fortunati et al. 2010; Echegoyen and Nerin 2013; Fortunati et al. 2013). Based on this fact, our long term goal is to develop a composite comprising hybrid cellulose-copper nanoparticles and thermoplastic resins with an effective copper release rate and appropriate mechanical properties for the use as antimicrobial material in active food packaging. Cellulose based materials we used in the research not only act as a template and stabilizer of copper nanoparticles, but also with the aim to provide an improved and controlled release of copper ions. We used commercial carboxymethyl cellulose (CMC) as template for in situ synthesizing a potent biocide copper nanoparticles in our preliminary research (Zhong et al. 2013). The results demonstrated that copper nanoparticles with the particle size ranging from 10- and $20 \mathrm{~nm}$ were synthesized on $\mathrm{CMC}$ using reducing agent sodium borohydride. The resulting hybrid materials have proven their antimicrobial effectiveness against the nonpathogenic surrogate of foodborne pathogen Escherichia coli.

Numerous thermal processing techniques have been proposed to incorporate antimicrobial materials in thermoplastic resins matrices, such as extrusion, blending and injection molding (Appendini and Hotchkiss 2002; Delgado et al. 2011; Fortunati et al. 2012a; Jokar et al. 2012, Bikiaris et al. 2013; Grigoriadou et al. 2013). Delgado et al. (2011) added cupric oxide $(\mathrm{CuO})$ and metallic copper $\left(\mathrm{Cu}^{0}\right)$ nanoparticles into polypropylene through a melt blending process followed by a press molding stage. The resulting composites, containing $5 \mathrm{v} / \mathrm{v} \%$ of nanoparticles, were able to inactivate more than $95 \%$ of the bacterium Escherichia coli after $4 \mathrm{~h}$ of direct contact. The authors demonstrated as well that nanoparticles of cupric oxide $(\mathrm{CuO})$ were more effective as antimicrobial material 
compared with nanoparticles of metallic copper $\left(\mathrm{Cu}^{0}\right)$. Fortunati et al. (2012a) developed an antimicrobial ternary composite film through the inclusion of cellulose nanocrystals and silver nanoparticles in PLA resin. The combination of $1 \mathrm{wt}$ \% silver nanoparticles and 5 wt.\% cellulose nanocrystals into the PLA matrix endowed composites with good antimicrobial properties against Staphylococcus aureus and Escherichia coli. In terms of the morphological properties of the PLA composites the authors concluded that aggregates of cellulose nanocrystals appeared in the composites after the extrusion process and therefore the tensile strength of those composites decreased as compared to pure PLA. Similarly, the melting temperature for PLA composites decreased compared to pure PLA. The reduction in melting temperature was explained based on changes in the crystalline structure of the matrices induced by cellulose nanocrystals and silver nanoparticles (Fortunati et al. 2012a; Fortunati et al. 2012b).

Jokar et al. (2012) added silver nanoparticles - embedded in polyethylene glycol (PEG) - in melted low density polyethylene (LDPE). The authors produced silver nanoparticles by the reduction of silver nitrate using polyethylene glycol (PEG) as reducing agent; PEG contributed positively to disperse copper nanoparticles in the thermoplastic matrix. The final nanocomposite films were able to reduce $57.8 \%$ of the Staphylococcus aureus growth rate.

Bikiaris et al. (2013) added copper nanofibers - prepared by reduction of a copper amine complex - as antimicrobial material into high density polypropylene (HDPE) films. The resulting copper nanofibers have a diameter of 200-400 $\mathrm{nm}$ and length of $15-25^{\mu} \mathrm{m}$. For an improved dispersion of copper nanofibers into HDPE matrix, the authors first physically premixed copper nanofibers and HDPE matrix using a ball mill device before feeding into the reomixer device; subsequently the authors used a melt mixing process, and the resulting HDPE composite films exhibited good antimicrobial properties against Escherichia coli DH5 $\alpha$, Pseudomonas fluorescens BS3 and Staphylococcus aureus strains. 
Grigoriadou et al. (2013) combined 2.5 wt.\% of copper nanfibers with high density polypropylene (HDPE) using a melt mixing process. Copper nanofibers were prepared similarly to those reported by Bikiaris et al. (2013), and the final nanocomposites resulted in the improvement in mechanical property. The tensile strength of the new nanocomposites were $8.5 \%$ higher than pure HDPE. The authors did not evaluate the antimicrobial properties of their composites.

Coating process is another methodology that was used to incorporate antimicrobial materials on thermoplastic resins (Del Nobile et al. 2004; Li et al. 2009). Del Nobile et al. (2004) developed an active polyethylene (PE) film that was prepared by depositing silver clusters via plasma. The effective inhibition of the growth of Alicyclobacillus acidoterrestris strain in apple juice medium containing $\mathrm{Ag}$ coated $\mathrm{PE}$ films was demonstrated.

Li et al. (2009) coated zinc oxide nanoparticles on polyvinyl chloride (PVC) films using a glass coater. The films with a $\mathrm{ZnO}$ nanoparticles concentration of $187 \mu \mathrm{g} / \mathrm{cm}^{2}$ resulted effective in the growing inhibition of Escherichia coli and Staphylococcus aureus.

As mentioned before, little work has been performed regarding the feasibility to release antimicrobial nanoparticles from thermoplastic resins (Fernandez et al. 2010; Echegoyen and Nerin 2013; Fortunati et al. 2013). Fernandez et al. (2010) studied the release of silver from polylactide/silver zeolites composites in different food simulants media at different temperatures. The authors concluded that the releasing capacity of silver ions was affected by the various composite processing for film formation, the types of media. The release kinetics of ions might also be as a result of the ambient temperature. The silver releasing capacity of films formed by solvent casting was higher than that of films formed by melt mixing. The releasing kinetic was greatly slower at $4{ }^{\circ} \mathrm{C}$ than that at $20{ }^{\circ} \mathrm{C}$ because the reduced molecular motion at $4{ }^{\circ} \mathrm{C}$ decrease the silver ion release from the matrix. The higher polarity and cations concentration in the media also could facilitate the silver ion release. Echegoyen and Nerin (2013) studied nanoparticles release from commercial nano- 
silver antimicrobial food containers and found that silver release ability was higher in the food simulant acetic acid $3 \mathrm{v} / \mathrm{v} \%$ than in food simulant ethanol $50 \mathrm{v} / \mathrm{v} \%$. The authors concluded that higher concentrations of cations and protons in the media could enhance silver release ability, which is consistent with Fernandez et al. (2010) conclusion. Fortunati et al. (2013) produced film nanocomposites using cellulose nanocrystals and silver nanoparticles embedded in PLA. The modified cellulose nanoparticles that have been surfactant modified by an acid phosphate ester of ethoxylated nonylphenol could result in a partial plasticizer effect of the matrix to increase diffusion of silver ions to aqueous simulants. The author evaluated the silver release from the PLA matrix and demonstrated that the release of silver ion from PLA composites containing $5 \mathrm{wt} \%$ surfactant modified cellulose nanocrystals and $1 \mathrm{wt} \%$ silver nanoparticles is greatly higher than the PLA composites only containing $1 \mathrm{wt}$ \% silver nanoparticles.

In the present research, we primarily investigated how the thermoplastic resins, PP and PLA perform in terms of their morphological, mechanical, thermal and antimicrobial properties when freeze-dried hybrid cellulose-copper nanoparticles are incorporated into the matrices. In addition, we determined the copper ion release from the matrices and their correspondent antimicrobial activity. The cellulose raw material used consisted of CMC, previously used by Zhong et al. (2013) and TNFC. Both cellulose-based materials were used as template for the synthesis of copper nanoparticles. We were expecting that TNFC's carboxylic groups' availability will promote an effective synthesis for copper nanoparticles. TEMPO-mediated oxidation followed by successive mild disintegration in water provide an effective way to produce nanofibers from native cellulose that are 3-4 $\mathrm{nm}$ wide and several microns in length (Isogai et al. 2011); a significant amount of carboxylate groups are expected to be introduced into native cellulose by TEMPO-mediated oxidation (Saito et al. 2007).

The cellulose-based materials (CMC and TNFC) were incorporated into the thermoplastic resins PP and PLA resins using extrusion-injection and extrusion-film formation. It is well known that PP has a lot of advantages such as ready availability, competitive price, good 
processability, high strength and high chemical stability that promoted PP to be used in the food industry for over 50 years (Hong et al. 2006; Emamifar et al. 2010; Allahvaisi 2012; Wu et al. 2012; Bikiaris et al. 2013). On the other hand, PLA has attracted much interest and is becoming a major sustainable alternative to petrochemical-based product because PLA is a bio-degradable thermoplastic polymer that can be produced from biomass readily available in large amount (Jonoobi et al. 2010; Nyambo et al. 2010; Frone et al. 2013). Both PP and PLA have been used in several applications such as food packaging, automotive and biomedical fields (Kale et al. 2006; Fages et al. 2011; Allahvaisi 2012; Bajpai et al. 2012; Fortunti et al. 2012a).

\subsection{Materials and methods}

\subsubsection{Materials}

Sodium carboxymethyl cellulose (CMC) (average molecular weight: 90,000) from Sigma Aldrich, USA.; technical-crystal cupric sulfate pentahydrate $\left(\mathrm{CuSO}_{4} \cdot 5 \mathrm{H}_{2} \mathrm{O}\right)$ from fisher Scientific, USA; sodium borohydride $\left(\mathrm{NaBH}_{4}\right)(0.5 \mathrm{M})$ from Acros Organics, USA.; ethanol (reagent alcohol, specially denatured alcohol formula 3A 95\% and isopropyl alcohol 5\% by volume) from Sigma Aldrich, USA.; Nitric acid (69\%-71\% w/w) from Fisher Scientific, USA.; TEMPO nanofibrillated cellulose (TNFC) (0.67 wt.\%) from the Forest Product Laboratory, Madison, WI; polypropylene (PP) (Certene PHM-20AN) from Muehlstein and Co., USA; polylactic acid (PLA) (Ingeo 4043D) from Nature Works LLC, USA; lubricant (Struktol TPW113) from Struktol Company of America, USA; maleic anhydride grafted polypropylene coupling agent (Eastman G-3015) from Eastman

Chemical Company, USA; nonpathogenic E. coli DH5 $\alpha$ from Invitrogen Inc., USA ( $\alpha$ substrain of DH5 described by Hanahan in 1985, "DH" stands for Douglas Hanahan) is a very sensitive microorganism. This $E$. coli contains mutations of the recA and gyrA (gyrase subunit A) genes that are necessary for DNA repair and replication. Therefore, recA and gyrA mutants have impaired ability to repair and recombine their DNA strands making the mutants sensitive to any stress including chemicals. This is why this sensitive 
and nonpathogenic E. coli was selected as a model microbial target in this study. Sterile trypticase soy broth (TSB) from Becton Dickinson, USA; Petrifilm E. coli/Coliform count plate from 3M, USA; Butterfield phosphate buffer from Hardy Diagnostics, USA.

\subsubsection{Preparation of the hybrid CMC-copper nanoparticles}

Table 5. 1 Preparation of the hybrid CMC-copper nanoparticles.

\begin{tabular}{ccccccc}
\hline $\begin{array}{c}\text { Na-CMC } \\
\text { solution } \\
\#\end{array}$ & $\begin{array}{c}\text { Sample } \\
\text { Codes }\end{array}$ & $\begin{array}{c}\text { Na-CMC } \\
(\mathrm{g})\end{array}$ & $\begin{array}{c}\text { Deionized } \\
\text { water } \\
(\mathrm{mL})\end{array}$ & $\begin{array}{c}\mathrm{CuSO}_{4} \\
\text { solution } \\
(0.1 \mathrm{~mol} / \mathrm{L}) \\
(\mathrm{mL})\end{array}$ & $\begin{array}{c}\text { Treated } \\
\text { with } \\
\text { washing } \\
\text { process. } \\
\text { Yes } / \mathrm{No}\end{array}$ & $\begin{array}{c}\mathrm{NaBH}_{4} \\
\text { solution } \\
(0.5 \\
\mathrm{mol} / \mathrm{L}) \\
(\mathrm{mL})\end{array}$ \\
\hline 1 & $\begin{array}{l}\mathrm{CMC}- \\
\mathrm{Cu}(1)\end{array}$ & 1 & 99 & $5^{\mathrm{a}}$ & Yes & 2 \\
2 & $\begin{array}{l}\mathrm{CMC}- \\
\mathrm{Cu}(2)\end{array}$ & 1 & 99 & $10^{\mathrm{b}}$ & Yes & 4 \\
3 & $\begin{array}{l}\mathrm{CMC}- \\
\mathrm{Cu}(3)\end{array}$ & 1 & 99 & $5^{\mathrm{a}}$ & No & 2 \\
4 & $\begin{array}{l}\mathrm{CMC}- \\
\mathrm{Cu}(4)\end{array}$ & 1 & 99 & $10^{\mathrm{b}}$ & $\mathrm{No}$ & 4 \\
\hline
\end{tabular}

CMC-Cu: carboxymethyl cellulose-copper nanoparticles

a - Expected copper concentration: $305^{\mu} \mathrm{g} / \mathrm{mL}$

b - Expected copper concentration: $582 \mu \mathrm{g} / \mathrm{mL}$

The general procedure used for the preparation of the hybrid carboxymethyl cellulosecopper nanoparticles has been described previously (Zhong et al. 2013). One gram of CMC was dissolved in $99 \mathrm{~mL}$ deionized water under vigorous magnetic stirring. Copper sulfate solution $(0.1 \mathrm{~mol} / \mathrm{L})$ was added by drops into the Na-CMC solutions (Table 5.1$)$. The mixtures were subjected to high-speed mixing while the copper sulfate solution was added. Then the mixtures were allowed to react at room temperature for $3 \mathrm{~h}$. Hybrids with copper

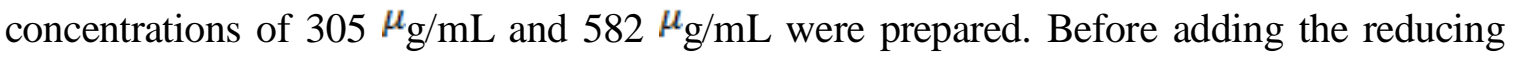
agent some of the mixtures were subjected to a washing stage (Table 5.1), where the excess of cupric ions were removed by placing the mixture into dialysis tubing. The washing process was performed with deionized water for $24 \mathrm{~h}$. For all mixtures, cupric ions were reduced to copper $\left(\mathrm{Cu}^{0}\right)$ or copper oxide nanoparticles by adding the reducing agent sodium borohydride. Hybrid carboxymethyl cellulose-copper nanoparticles solutions were 
subsequently dried on a VirTis Genesis Freeze Dryer (Warminster, USA) for 1 week. The solid hybrid material with two different copper concentration was grinded to powder using a Waring Commercial Blender for $10 \mathrm{~min}$.

\subsubsection{Preparation of the hybrid TNFC-copper nanoparticles}

A $0.8-\mathrm{mL}$ aliquot of cupric sulfate solution $(0.1 \mathrm{~mol} / \mathrm{L})$ was added by drops into sixteen grams of TNFC gel (0.67 wt.\%). The mixture of TNFC and cupric sulfate was subjected to high-speed mixing while the cupric sulfate solution was added. Then the mixture was allowed to react at room temperature for $3 \mathrm{~h}$. After that, cupric ions were reduced to copper nanoparticles by adding, while stirring, $0.16 \mathrm{~mL}$ of reducing agent sodium borohydride. The powders of hybrid TNFC-copper nanoparticles composite materials were obtained using freeze-drying followed by grinding process as described above.

\subsubsection{Preparation of composites: hybrid cellulose-copper nanoparticles embedded in thermoplastic resins}

PP and PLA pellets were dried in an oven overnight at $105^{\circ} \mathrm{C}$. The resulting hybrids of cellulose-copper nanoparticles were incorporated into the thermoplastic resins PP and PLA using extrusion-injection and extrusion-film processes. Extrusion was performed using a DSM Xplore twin-screw micro-extruder and injection process was performed using a DSM 10cc Micro Moulding Machine (Geleen, Netherlands). The operation settings for the composites preparation through extrusion-injection processes are presented in Table 5.2.

Polypropylene and maleic anhydride grafted polypropylene coupling agent were fed into the DSM Xplore micro-extruder and melted/mixed for $180 \mathrm{~s}$; then the hybrid cellulosecopper nanoparticles in mixture with a lubricant were fed into the micro-extruder allowing them to blend with the thermoplastic resin for $180 \mathrm{~s}$. At the end of the blending period of $180 \mathrm{~s}$, the composite mixture was transferred into the moulding-injection machine to obtain the extruded-injected specimens. The same general procedure was utilized when PLA was 
used as matrix; only differences in the processing temperatures were established (Table 5.2). Sample codes and mass percentages of the composite's components are presented in Table 5.3 and Table 5.4, respectively. In addition, preliminary PLA films embedded with the hybrid cellulose-copper nanoparticles were prepared by using DSM Xplore twin-screw micro-extruder followed by DSM Film Device Machine (Geleen, Netherlands).

Table 5. 2 Processing setting parameters for PP and PLA extruded-injected composites.

\begin{tabular}{lcc}
\hline DSM Xplore micro-extruder parameters & PP & PLA \\
\hline Screw speed (rpm) & 100 & 100 \\
Temperature Profile $\left({ }^{\circ} \mathrm{C}\right)$ & $180-180-180$ & $190-190-190$ \\
Mixing time (s) & 180 & 180 \\
& & \\
\hline Injection moulding setting parameters & & 50 \\
\hline Temperature of mould $\left({ }^{\circ} \mathrm{C}\right)$ & 25 & 195 \\
Temperature of transfer cylinder $\left({ }^{\circ} \mathrm{C}\right)$ & 180 & 15 \\
Injection and holding pressure $($ bar $)$ & 8 & \\
\hline
\end{tabular}

Table 5. 3 Mass percentage of the thermoplastic resin PP and the hybrid cellulose- copper nanoparticles in the composite production.

\begin{tabular}{ccccccc}
\hline & \multicolumn{7}{c}{ Sample Codes } \\
\cline { 2 - 7 } Ingredients & & PP/ & PP/ & PP/ & PP/ & PP/ \\
& & CMC- & CMC- & CMC- & CMC- & TNFC- \\
\hline PP & $100 \%$ & $93 \%$ & $93 \%$ & $93 \%$ & $93 \%$ & $\mathrm{Cu}$ \\
\hline Coupling agent & - & $3 \%$ & $3 \%$ & $3 \%$ & $3 \%$ & $93 \%$ \\
Lubricant & - & $2 \%$ & $2 \%$ & $2 \%$ & $2 \%$ & $3 \%$ \\
CMC-Cu (1) & - & $2 \%$ & - & - & - & $2 \%$ \\
CMC-Cu (2) & - & - & $2 \%$ & - & - & - \\
CMC-Cu (3) & - & - & - & $2 \%$ & - & - \\
CMC-Cu (4) & - & - & - & - & $2 \%$ & - \\
TNFC-Cu & - & - & - & - & - & $2 \%$ \\
\hline
\end{tabular}

CMC-Cu: carboxymethyl cellulose-copper nanoparticles CNF-

$\mathrm{Cu}$ : cellulose nanofibers-copper nanoparticles 
Table 5. 4 Mass percentage of the thermoplastic resin PLA and the hybrid cellulosecopper nanoparticles in the composite production.

\begin{tabular}{ccccccc}
\hline & \multicolumn{6}{c}{ Sample Codes } \\
\cline { 2 - 7 } Ingredients & PLA & PLA/ & PLA/ & PLA/ & PLA/ & PLA/ \\
& & CMC- & CMC- & CMC- & CMC- & TNFC- \\
& & $\mathrm{Cu}(1)$ & $\mathrm{Cu}(2)$ & $\mathrm{Cu}(3)$ & $\mathrm{Cu}(4)$ & $\mathrm{Cu}$ \\
\hline PLA & $100 \%$ & $93 \%$ & $93 \%$ & $93 \%$ & $93 \%$ & $93 \%$ \\
Coupling agent & - & $3 \%$ & $3 \%$ & $3 \%$ & $3 \%$ & $3 \%$ \\
Lubricant & - & $2 \%$ & $2 \%$ & $2 \%$ & $2 \%$ & $2 \%$ \\
CMC-Cu(1) & - & $2 \%$ & - & - & - & - \\
CMC-Cu(2) & - & - & $2 \%$ & - & - & - \\
CMC-Cu(3) & - & - & - & $2 \%$ & - & - \\
CMC-Cu(4) & - & - & - & - & $2 \%$ & - \\
TNFC-Cu & - & - & - & - & - & $2 \%$ \\
\hline
\end{tabular}

CMC-Cu: carboxymethyl cellulose-copper nanoparticles

$\mathrm{CNF}-\mathrm{Cu}$ : cellulose nanofibers-copper nanoparticles

\subsubsection{Field emission scanning electron microscopy-energy dispersive X-ray spectroscopy characterization (FESEM-EDX)}

The morphological and copper elemental mapping analyses of the hybrid cellulose-copper nanoparticles materials were analyzed using a Hitachi S-4700 FESEM with an EDAX brand Energy dispersive spectroscopy detector (Tokyo, Japan). To obtain clean and precise fracture surfaces, all composites samples were cryo-fractured by immersing the specimens in liquid nitrogen and breaking them manually. Before FESEM observation all fractured surfaces were coated with gold-platinum using the sputtering process.

\subsubsection{Mechanical strength-tensile testing}

The tensile strength of the extruded-injected composites were tested using an Instron 810 Material Test System (Norwood, USA) according to the ASTM D638 standard. The tensile tests were performed with a cell load of $2.5 \mathrm{kN}$ and a cross head speed of $5 \mathrm{~mm} / \mathrm{min}$. Five specimens were measured for each material and the average value and correspondent standard deviation were determined. 


\subsubsection{Differential scanning calorimetry (DSC) characterization}

The thermal properties of the produced composites was measured using a TA Instruments Q20 differential scanning calorimeter with refrigerated cooling system (New Castle, USA). The temperature of analysis were from $25^{\circ} \mathrm{C}$ to $250{ }^{\circ} \mathrm{C}$ with a heating ram-up rate of 10 ${ }^{\circ} \mathrm{C} / \mathrm{min}$. The glass transition temperature $\left(T_{g}\right)$, cold crystallization temperature $\left(T_{c c}\right)$, melting temperature $\left(T_{m}\right)$, cold crystallization and melting enthalpies $\left(\Delta H_{c c}, \Delta H_{m}\right)$ were measured from the first heating scan.

The degree of crystallinity of PP composites was determined according to equation (1) (Bahar et al. 2012):

$$
X_{c}=\left[\Delta H_{m} /\left(\Delta H_{m 0} \times w\right)\right]^{\times} 100 \% \quad \text { Eq. (1) }
$$

where $\Delta H_{m}$ is the enthalpy of melting the sample measured by DSC, $\Delta H_{m 0}$ is the enthalpy of melting for $100 \%$ crystalline PP (value of $209 \mathrm{~J} / \mathrm{g}$ as reported by Fan et al. (2011)), and $w$ is the mass fraction of PP in the sample.

The degree of crystallinity of PLA composites was calculated according to equation (2) (Fortunati et al. 2010):

$$
X_{c}=\left[\left(\Delta H_{m}-\Delta H_{c c}\right) /\left(\Delta H_{m 0} \times w\right)\right]^{\times} 100 \% \text { Eq. (2) }
$$

where $\Delta H_{c c}$ is the enthalpy of cold crystallization and $\Delta H_{m}$ is the enthalpy of melting the sample, both were measured by DSC; $\Delta H_{m 0}$ is the enthalpy of melting for $100 \%$ crystalline PLA (value of $93 \mathrm{~J} / \mathrm{g}$ as reported by Fisher et al. (1973) and Tsuji et al. (1996)), and $w$ is the mass fraction of PLA in the sample. PLA displays a cold crystallization peak above the glass transition temperature and this value is put in the formula used to determine its crystallinity. 


\subsubsection{Atomic absorption spectroscopy (AAS) characterization of copper ion release from thermoplastic composites in an aqueous medium}

Extruded-injected specimens prepared from extrusion and injection molding processes were stored in bottles containing $100 \mathrm{~mL}$ deionized water so as to monitor the copper ion release from the produced composites. The analytes collected from such storages were characterized using a Perkin-Elmer 2380 atomic absorption spectrometer (AAS) (Waltham, USA). The quantification of copper ions released from the specimens were measured at one-week interval for a month.

A rectangular piece from PLA/TNFC-Cu composites was cut to get the following dimensions: length $100 \mathrm{~mm}$ and width $30 \mathrm{~mm}$. Two pieces of PLA/TNFC-Cu composite were stored into the $100 \mathrm{~mL}$ of deionized water. The copper ions leaching into media were analyzed for quantification of copper ions release from the films at one-week interval for a month using AAS.

\subsubsection{Antimicrobial properties of composite film of PLA containing freeze-dried hybrid TNFC-copper nanoparticles}

E. coli lyfo-disks were reconstituted by crushing one pellet using a sterile spatula in $0.5 \mathrm{~mL}$ of sterile LTB. The content was aseptically transferred to $99.5 \mathrm{~mL}$ of sterile LTB and

allowed to grow aerobically at $\quad 37^{\circ} \mathrm{C}$ for $24 \mathrm{~h}$ in an incubator/shaker set at $150 \mathrm{rpm}$ (C24, New Brunswick Scientific, New Jersey, USA). This procedure yielded $100 \mathrm{~mL}$ of E. coli stock culture. For experiments, a loopful of the stock culture was transferred to 100 $\mathrm{mL}$ of sterile TSB followed by incubation at $37^{\circ} \mathrm{C}$ for $24 \mathrm{~h}$ in the $\mathrm{C} 24$ incubator/shaker set at $150 \mathrm{rpm}$. This procedure yielded a culture with appropriately $10^{8}$ colony-forming units per milliliter (CFU/mL). A 2-mL aliquot of such culture was transferred to a surface of PLA/TNFC-Cu thin film and pure PLA thin film and incubated at room temperature for $1 \mathrm{wk}$. To prevent excessive evaporation of the E. coli culture from the 
surface of films, the 
films were kept in an aerobic environment with saturated humidity. After given exposure time, $1 \mathrm{~mL}$ of $E$. coli culture was removed from the surface of films to enumerate $E$. coli survivors. Prior to removal, the E. coli culture on films was carefully mixed to obtain an equal cell distribution. Enumeration was performed by a standard serial 10-fold dilution procedure and spread plating in a bio-safety cabinet under aseptic procedures (Black and Jaczynski 2006; Levanduski and Jaczynski 2008). A 1-mL aliquot of the E. coli culture removed from the surface of films was aseptically mixed with $9 \mathrm{~mL}$ of diluent (Butterfield phosphate buffer) followed by shaking the diluent bottle to uniformly distribute bacterial cells. Subsequent serial 10-fold dilutions were aseptically made by taking $10 \mathrm{~mL}$ of diluted sample and transferring it to a $90-\mathrm{mL}$ diluent bottle. Survivors were enumerated on selective medium (Petrifilm ${ }^{\mathrm{TM}}$ E. coli/Coliform Count Plate) using a standard spreadplating technique. A 1.0-mL aliquot of each serial 10-fold dilution was pipetted and spread on $3 \mathrm{M}^{\mathrm{TM}}$ Petrifilm ${ }^{\mathrm{TM}}$ plates. The $3 \mathrm{M}^{\mathrm{TM}}$ Petrifilm ${ }^{\mathrm{TM}}$ plates were incubated at $35^{\circ} \mathrm{C}$ for $48 \mathrm{~h}$ (AOAC 1995, method 991.14).

\subsection{Results and discussion}

\subsubsection{Morphological and copper elemental mapping analysis of the hybrid cellulose- copper nanoparticles}

The morphology of the hybrid CMC-copper nanoparticles and TNFC-copper nanoparticles is presented in Figure 5.1a, 5.1b, respectively. As mentioned before, the dry hybrid materials were obtained through a freeze-drying process followed by a grinding procedure. The available grinder allowed the production of big particles (flake form of approximately 100 $\mu \mathrm{m}$ length), which most probably are having cellulose aggregates difficult to be separated. It is well known that cellulose have a great tendency to aggregate when dried because of their high surface area and strong hydrogen bonds formation after water sublimation (Jonoobi et al. 2010; Fortunati et al. 2012b). Figure 5.1c and 5.1d display the map distribution of copper on both CMC and TNFC. In those pictures copper is represented by tiny light dots and according to the picture they were distributed very homogeneously on the cellulose surfaces. 

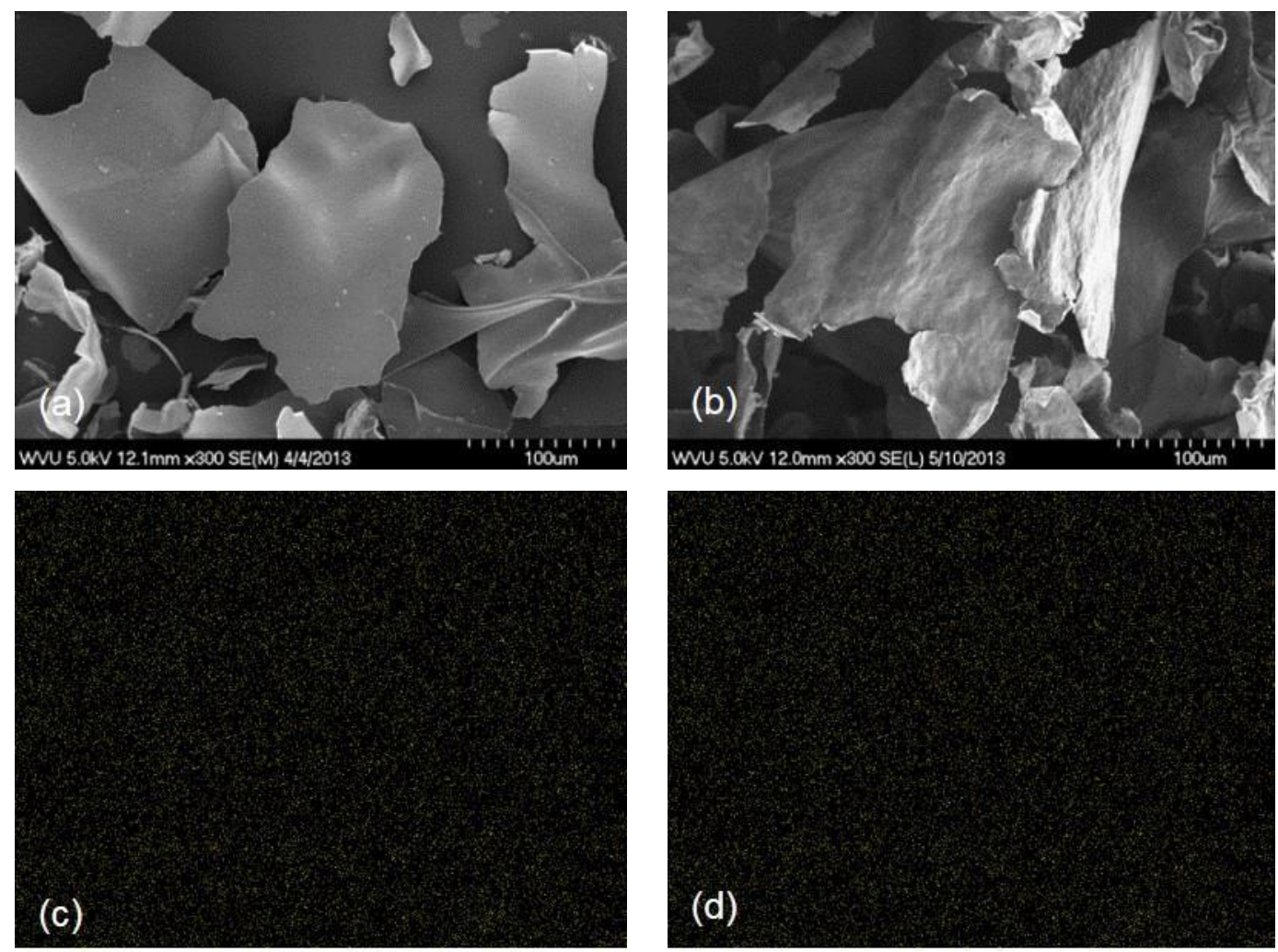

Figure 5. 1 FESEM-EDX characterization of (a) hybrid CMC-copper nanoparticles and (b) hybrid TNFC-copper nanoparticles and their corresponding copper elemental mapping images of (c) hybrid CMC-copper nanoparticles and (d) hybrid TNFC-copper nanoparticles (Tiny light dots represent copper element).

\subsubsection{Microstructure of the hybrids CMC-copper nanoparticles and TNFC-copper nanoparticles embedded in thermoplastic resins (extruded-injected specimens)}

Figure 5.2 displays the cryo-fractured surfaces of pure PP and PP composites. Some of the hybrid material (carboxymethyl cellulose-copper nanoparticles) remained as aggregates, as observed in Figure 5.2d, 5.2e (inside black circle). In Figure 5.2b, 5.2c, 5.2f, it is not possible to identify aggregates; apparently the hybrid material is coated with the polymer generating good adhesion between the composite's components. However, even though several pictures were taken, they are not enough to predict any mechanical property. As observed in Figure 5.3 at lower magnification, pure PLA seems to have relatively smooth and compacted surface than the correspondent PLA composites; however from pictures of 
PLA and PLA composites at higher magnification (Figure 5.4), a porous structure is observed. Similar results in which cellulose nanomaterials have imparted rough fracture surfaces and voids have been shown elsewhere (Srithep et al. 2013). The rough surface is likely caused by aggregate TNFC-polymer bundles that are not readily broken. The voids might be as a result of debonding between the cellulose and polymer and possibly even as a result of residual moisture vaporizing and creating voids in the polymer.
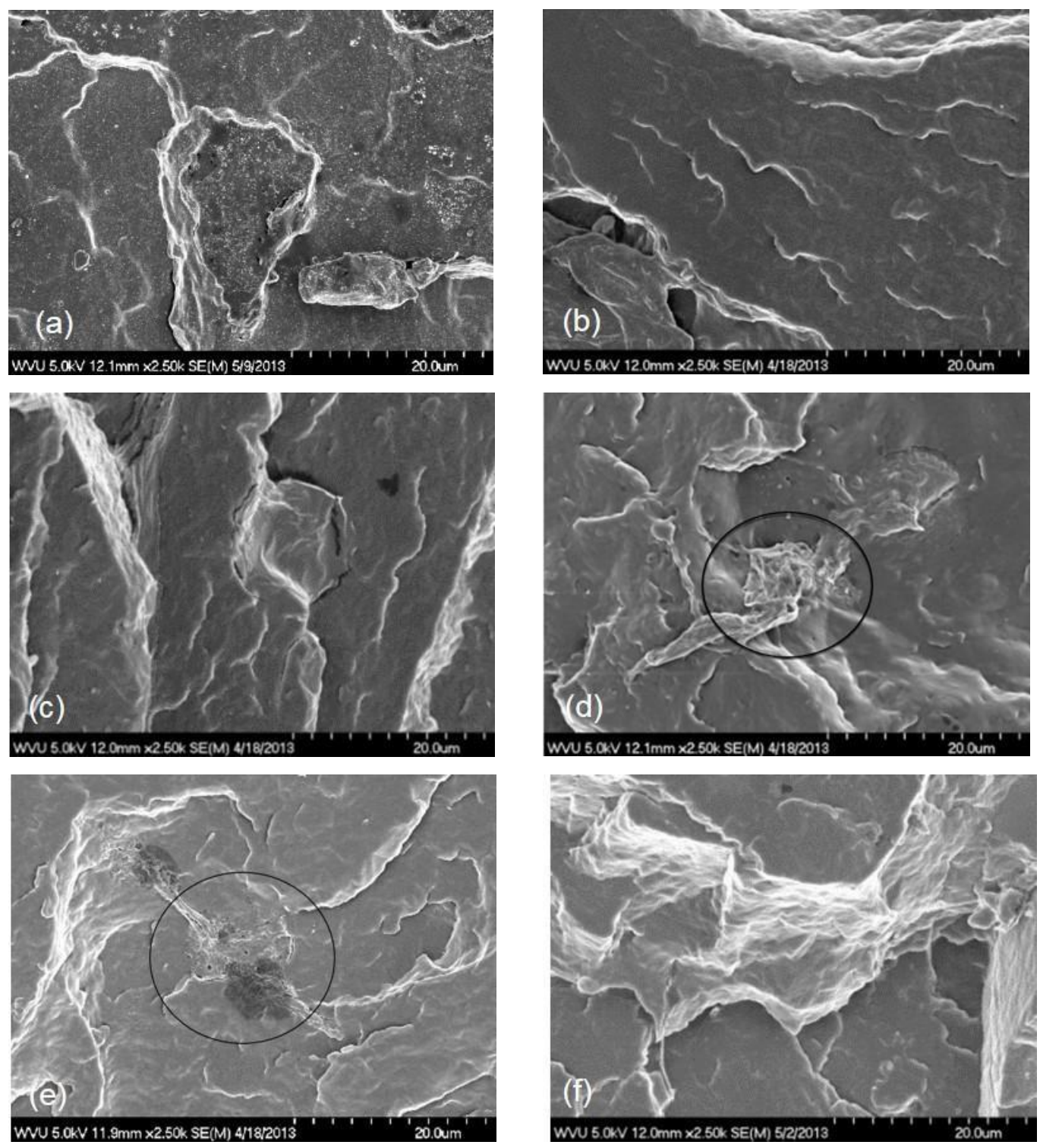

Figure 5. 2 SEM images of cryo-fractured surfaces of (a) PP, (b) PP/CMC-Cu (1), (c) PP/CMC-Cu (2), (d) PP/CMC-Cu (3), (e) PP/CMC-Cu (4), (f) PP/TNFC-Cu. 

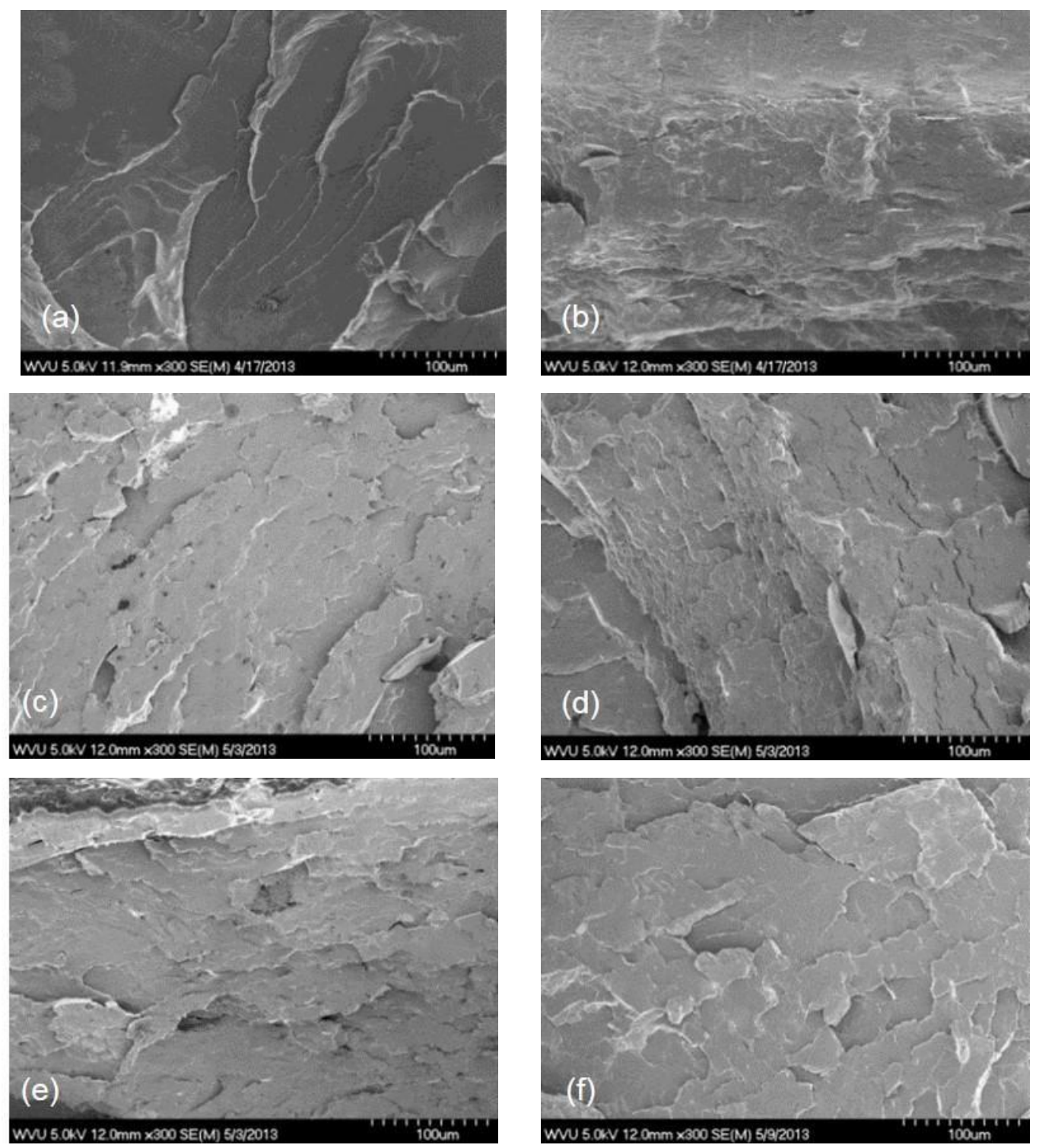

Figure 5. 3 SEM images of cryo-fractured surfaces of (a) PLA, (b) PLA/CMC-Cu (1), (c) $\mathrm{PLA} / \mathrm{CMC}-\mathrm{Cu}$ (2), (d) PLA/CMC-Cu (3), (e) PLA/CMC-Cu (4), (f) PLA/TNFC-Cu. 

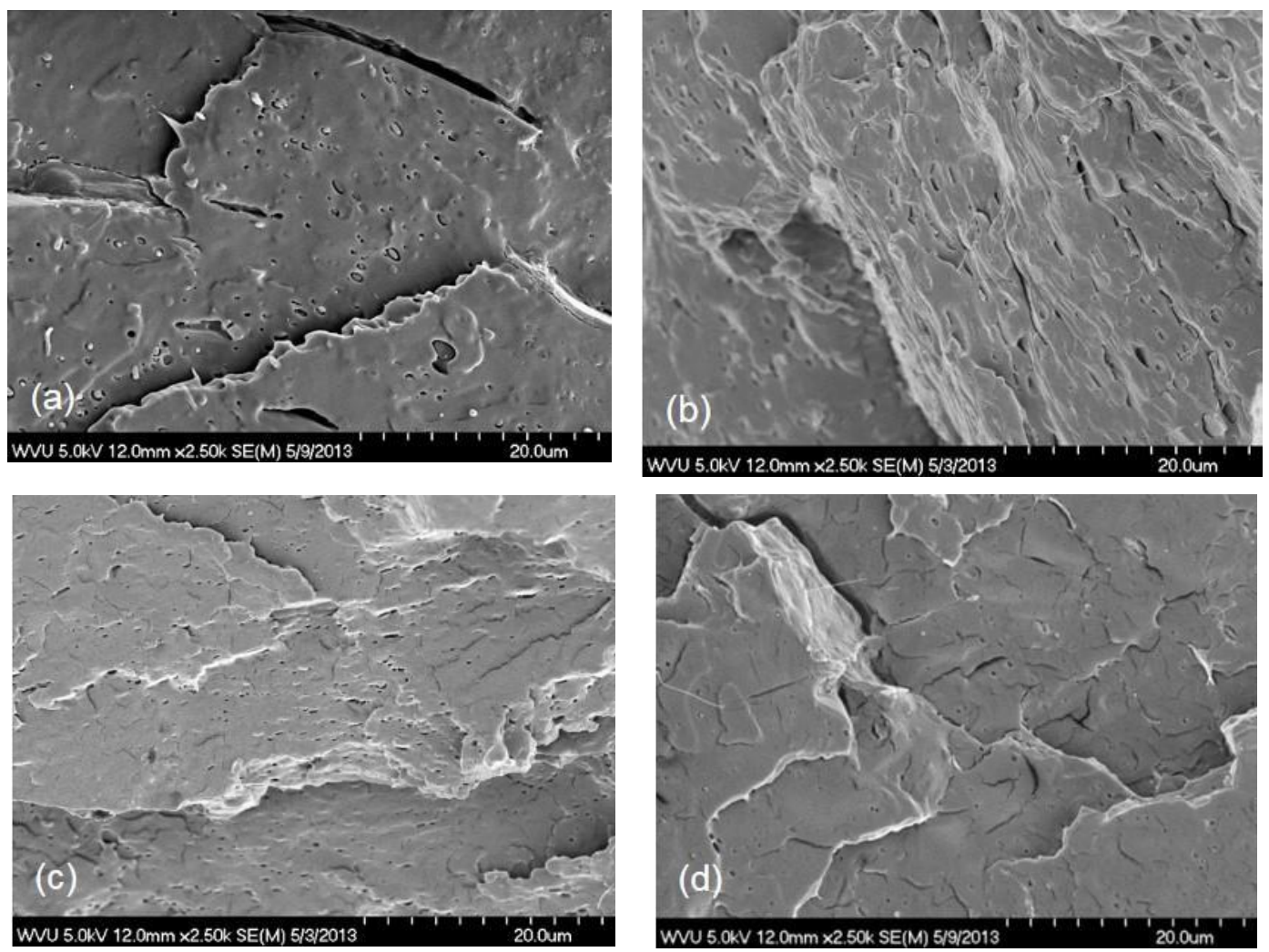

Figure 5. 4 SEM images of cryo-fractured surfaces of (a) PLA, (b) PLA/CMC-Cu(3), (c) PLA/CMC-Cu(4), (d) PLA/TNFC-Cu at higher magnification.

\subsubsection{Mechanical strength-tensile testing on the hybrids CMC-copper nanoparticles and TNFC-copper nanoparticles embedded in thermoplastic resins (extruded- injected specimens)}

Tensile strength resulted higher for pure PLA and its composites, compared to pure PP and its composites (Figure 5.5). All composites display tensile strength lower than the correspondent pure matrix, except for PLA/CMC-Cu (3) in which case there is no statistical difference between the composite and the pure matrix. It is observed from Figure 5.5a, 5.5b that for both PP and PLA composites, higher concentrations of copper in the hybrid material (solution number 2 and 4 from Table 5.1) resulted in slightly lower tensile strength values compared with lower copper concentrations. That is, for PP composites prepared with the higher copper concentration in the hybrid material (solutions 2 and 4), the tensile strength decreased up to $16.9 \%$ and $19.2 \%$, respectively. 

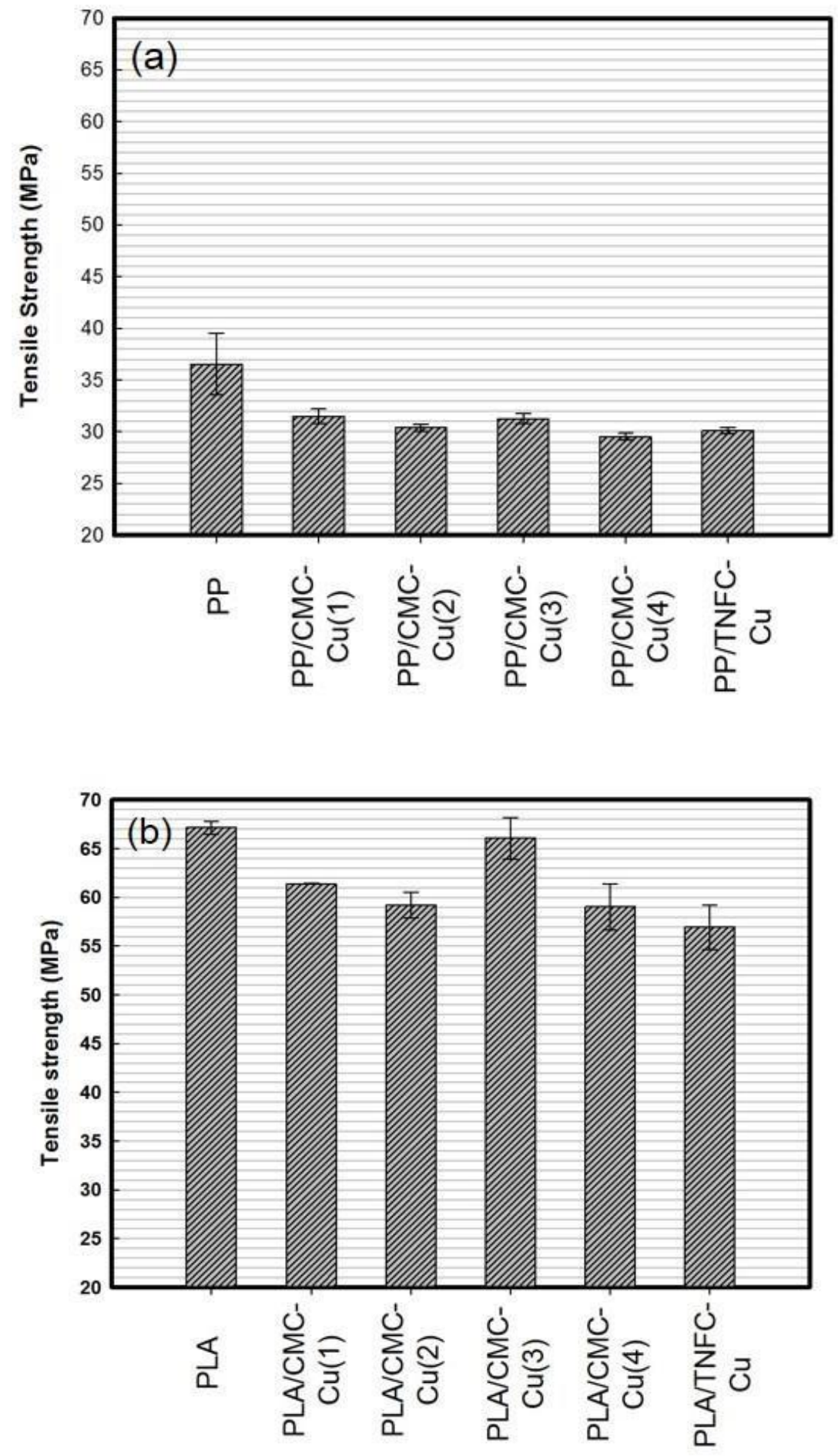

Figure 5. 5 Tensile strength of (a) PP composites and (b) PLA composites. 
For those PP composited prepared with lower copper concentrations in the hybrid material (solutions 1 and 3), the tensile strength was reduced up to $13.8 \%$ and $14.5 \%$, respectively. A reduced effect of copper concentration for PLA composites compared with PP composites was observed. That is, for PLA composites prepared with the higher copper concentration in the hybrid material (solutions 2 and 4) the tensile strength was reduced up to $11.8 \%$ and $12.1 \%$, respectively. For those PLA composites prepared with the lower copper concentrations in the hybrid material (solutions 1 and 3), the tensile strength was reduced in average up to $8.6 \%$ and $1.6 \%$, respectively.

For composites prepared with PP and PLA plus TNFC-Cu, same concentration of copper component in the hybrid material, the reduction in the tensile strength was in average up to $17.6 \%$ and $15.2 \%$, respectively.

Copper concentrations is apparently reducing the tensile strength of the composites, but most importantly, the reduction in tensile strength might be as a result of the formation of cellulose agglomerates, porous and debondings between cellulose based materials and matrices. Aggregated materials might create stress concentration zones which in turn can act as a crack initiator (Fortunati et al. 2012a; Kazmiruk et al. 2012). It widely reported that tensile strength of composite material is mainly governed by the interfacial adhesion between cellulose based materials and matrices (Oksman et al. 2003; Bajpai et al. 2012).

\subsubsection{Thermal properties of hybrid copper nanoparticles and cellulose based materials in thermoplastic resins (extruded-injected specimens)}

Figure 5.6a and 5.6b display the thermal properties (melting temperature, glass transition temperature and the cold crystallization peak) for PP, PLA and their correspondent composites. The melting temperature of PP and PLA composites slightly reduced when compared to pure PP as shown in Figure 5.6a and 5.6b; this result might be as a consequence of the formation of more defective and less ordered crystals that resulted from the heterogeneous nucleation in both composites. Similar observations have been reported 
by other authors; melting temperature depends on the size and perfection of the crystalline lamellae (Liu and Donovan 1995; Mathew et al. 2006; Fortunati et al. 2012b; Paleo et al. 2013).
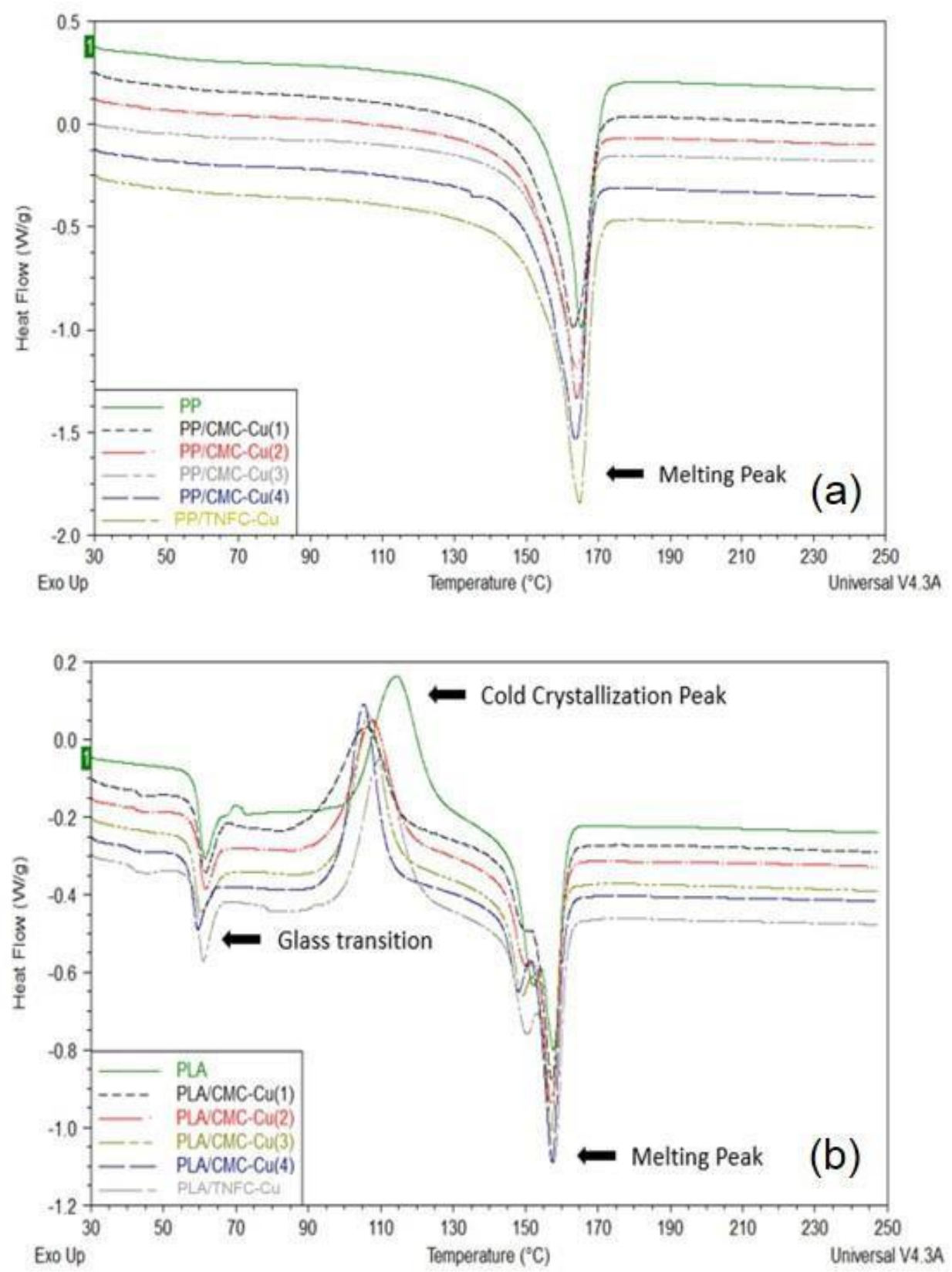

Figure 5. 6 DSC analysis for (a) PP and PP composites, and (b) PLA and PLA composites. 
A slight reduction of the glass transition temperature when PLA was embedded with the hybrids $\mathrm{CMC}-\mathrm{Cu}$ and TNFC-Cu was determined. This reduction might be as a consequence of the formation of more defective and less order crystals that made polymer chains move easily in a free volume. Two peaks of melting temperature are observed in Figure 5.6b for PLA and its composites. Each melting peak might correspond to different crystalline lamellae population essentially in terms of its thickness or its perfection or the meltingrecrystallization-melting mechanism (Avila-Orta et al. 2007; Yasuniwa et al. 2008; Frone et al. 2013).

A sharp endothermic peak linked to the glass transition of PLA is noticeable in Figure 5.6b. The peak is typically caused by stress relaxation on heating of PLA and was similarly observed by other researcheres (Pluta et al. 2002; Fortunati et al. 2010). A slight reduction of the glass transition temperature might also be attributed to the addition of hybrids CMC$\mathrm{Cu}$ and TNFC-Cu in the PLA matrix. As happened to PP composites, the slight reduction in glass transition temperature of PLA composites might be due to the formation of more defective and less ordered crystals caused by heterogeneous nucleation in PLA composites.

The cold crystallization peaks for PLA composites shifted to lower temperature when compared to the cold crystallization of pure PLA (Figure 5.6b). The lower of cold crystallization temperature could represent that faster crystallization took place, which might be induced by carboxymethyl cellulose-copper nanoparticles and cellulose nanofiberscopper nanoparticles. Cellulose based materials and metal nanoparticles could act as nucleating agents so as to enhance crystallization, and thereby decreasing the cold crystallization temperature; similar observations regarding this presented by the addition of metal nanoparticles have been reported by Fages et al. (2011) and Jiang et al. (2012). Also, hem fibers, wood flour, microcrystalline cellulose and cellulose nanofibers have been demonstrated to favor nucleation and the growth of crystals in thermoplastic resins (Mathew et al. 2006; Masirek et al. 2007; Lezak et al. 2008; Frone et al. 2013).

The degree of crystallinity of PP and PLA composites is presented in Figure 5.7. The results revealed that there is an increase in terms of the degree of crystallinity for both PP and PLA 
composites when compared to pure PP and PLA, respectively. As mentioned above, CMC and TNFC used in our case also might act as nucleating agents, and thereby contributing to the increased crystallinity of composites for both PP and PLA matrices. The increase in crystallinity of PLA after the incopration of cellulose based materials have also been reported previousl by Mathew et al. (2006). They reported that the crystallinity was increased by $130 \%$ after the addition of $25 \mathrm{wt} . \%$, of wood flour (particles size: $20-30 \mu \mathrm{m}$ ) in PLA matrices.

For PP composite prepared with hybrid materials that were treated by a washing process (solution 1 and 2), the increased in the degree of crystallinity up to $22.5 \%$ was observed for both cases. For those PP composites prepared with the hybrid materials without washing treatment (solution 3 and 4), the degree of crystallinity was brought up to $4.9 \%$ and $8.4 \%$, respectively. An increased effect of washing treatment for the PLA hybrid materials on the crystallinity compared with PP composites was also observed. For PLA composite prepared with hybrid materials that were treated by a washing process (solution 1 and 2), the degree of crystallinity was brought up to $356.2 \%$ and $393.8 \%$, respectively. For those PLA composites prepared with the hybrid materials without washing treatment (solution 3 and 4), the degree of crystallinity was brought up to $268.8 \%$ and $250.0 \%$, respectively. Washing process can remove excess of copper on the cellulose surface, and therefore copper nanoparticles might have a better ability to favor the nucleation and the growth of crystals in the thermoplastic resins. For composites prepared with PP and PLA embedded with TNFC$\mathrm{Cu}$, same concentration of copper component in the hybrid materials but without washing treatment, the increase in the degree of crystallinity was brought up to $24.9 \%$ and $106.2 \%$, respectively. In addition, the hybrid materials both $\mathrm{CMC}-\mathrm{Cu}$ and $\mathrm{TNFC}-\mathrm{Cu}$ acting as nucleation agents seems to have higher ability to enhance the crystallinity in PLA matrix in comparison with PP matrix. 

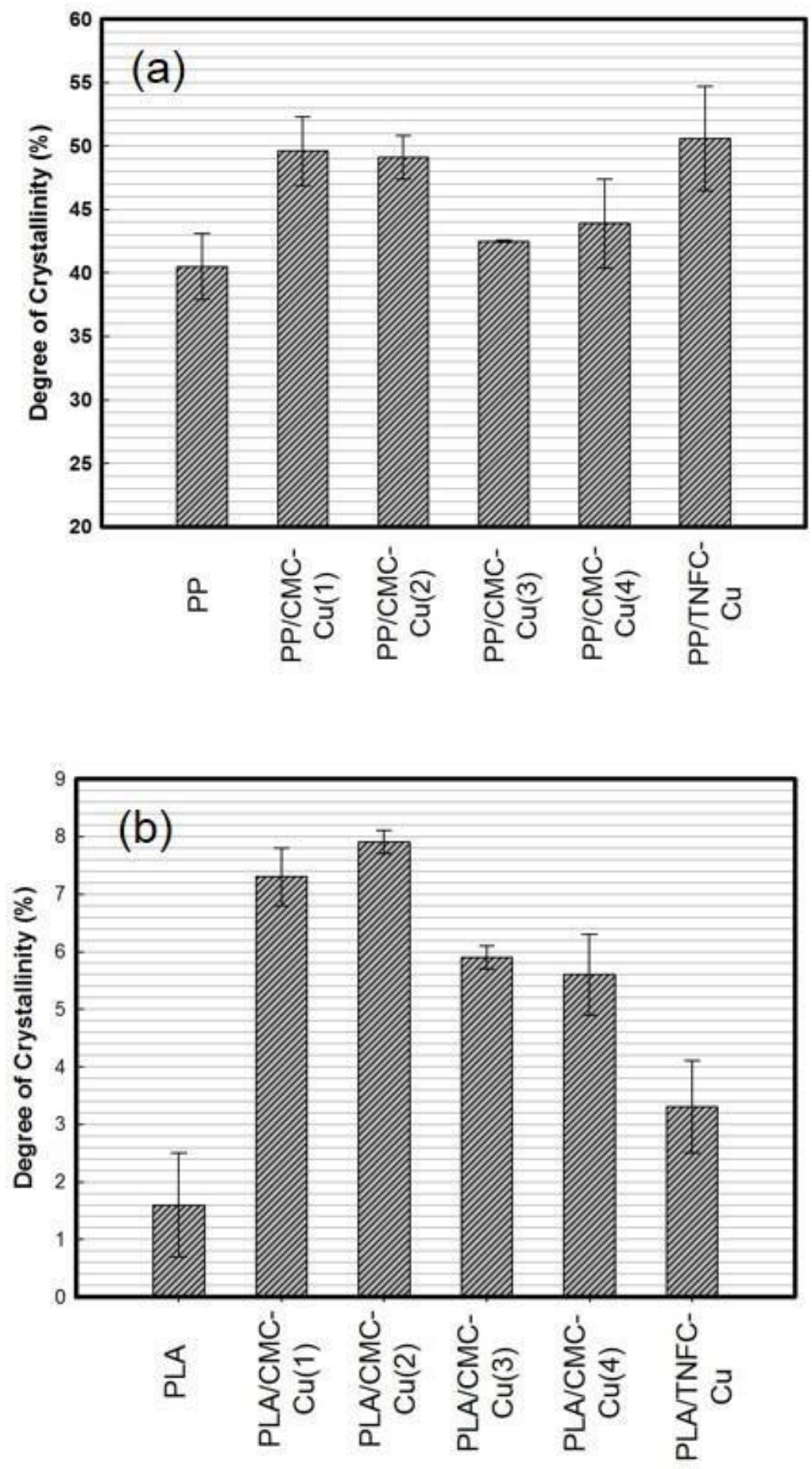

Figure 5. 7 Degree of crystallinity of (a) PP composites and (b) PLA composites. 


\subsubsection{Copper ion release from extruded-injected composites and film composites in aqueous medium}

Copper ion release from the extruded-injected composites (PP and PLA composites) was evaluated and copper ion leaching from the extruded-film formation composites (PLA composites) was also measured.

Copper ions were not found in the aqueous media in which extruded-injected specimens were immersed over one month. That might be as a result of the fact that the hybrid material was distributed in the whole composite but little copper was available on the surfaces. The thickness of those extruded-injected composites (about $1.5 \mathrm{~mm}$ ) might retard copper ions leaching from the matrices. In terms of the evaluation of copper release from PLA films (thickness: about $0.2 \mathrm{~mm}$ ) it is important to mention that just for those composites fabricated with the hybrid TNFC-copper nanoparticles, copper ions release was found (Table 5.5). Copper ions were detected in the aqueous solutions after 2-day immersion and the copper release lasted for over a period of a month.

Table 5. 5 Copper ions released from PLA film composites.

\begin{tabular}{cc}
\hline Time (days) & Copper ion concentration (ppm) \\
\cline { 2 - 2 } & PLA/TNFC-Cu \\
\hline 2 & 0.29 \\
9 & 0.42 \\
16 & 0.49 \\
30 & 0.62 \\
\hline
\end{tabular}

\subsubsection{Antimicrobial properties of pure PLA film and PLA/TNFC-Cu film against E.coli DH5 $\alpha$}

Since no copper ions leaching from those extruded injected composites were found, we only evaluated extruded-film composites (PLA/TNF-Cu) in terms of their antimicrobial properties. 
E.coli DH5 $\alpha$ culture with the initial concentration $1.9^{\times} 10^{8} \mathrm{CFU} / \mathrm{mL}$ were used as target microorganism in this study. The results of the antimicrobial tests of pure PLA film and PLA/TNFC-Cu film against E.coli $\mathrm{DH} 5 \alpha$ are presented in Table 5.6. The number of viable E.coli culture from the sample surfaces for both PLA film and PLA/TNFC-Cu film slightly increased with the increase in the exposure time, indicating both PLA film and PLA/TNFC-Cu film did not possess antimicrobial activity against E.coli DH5 $\alpha$. It has been reported that the hybrid cellulose-copper nanoparticles demonstrated excellent antimicrobial activity against E.coli DH5 (Zhong et al. 2013) and even though few copper ions ware detected from some of the film composites, the reason why these composite films did not show antimicrobial properties might be as a consequence of the low copper concentration leaching from the films. The amount of copper ions leaching from the film might not enough to result in inactivation of $E$. coli.

Table 5. 6 Antimicrobial results for PLA film and PLA/TNFC-Cu film against E.coli DH5 $\alpha$ under different exposure time.

\begin{tabular}{ccc}
\hline Exposure Time & \multicolumn{2}{c}{ Concentration of E.coli culture (CFU/mL) } \\
\cline { 2 - 3 } & PLA (Control) & PLA/TNFC-Cu \\
\hline $2 \mathrm{~h}$ & $2.0^{8} \times 10^{8}$ & $2.2 \times 10^{8}$ \\
1 week & $4.6 \times 10^{8}$ & $2.3 \times 10^{8}$ \\
2 weeks & $7.8 \times 10^{8}$ & $5.1 \times 10^{8}$ \\
\hline \hline
\end{tabular}

\subsection{Conclusions}

The incorporation of freeze dried hybrid cellulose-copper nanoparticles in both PP and PLA matrices was detrimental to their tensile strength. The reduction was greatly higher in those composites prepared with PP (19.2\%) than that in PLA (12.1\%). Even though copper concentration has apparently adverse effect on the tensile strength of the composites, the presence of fiber agglomerates in the matrices inducing the poor interfacial adhesion between fillers and matrices might be the most important factors for this mechanical property reduction. The agglomerations might be formed mainly during the drying freeze process. 
The melting temperature and glass transition temperatures slightly decreased for both PP and PLA composites after the incorporation of the hybrid materials in comparison with the pure matrices.

This result might be as a consequence of the formation of more defective and less ordered crystals due to heterogeneous nucleation in both composites. Similar effect might also lead to the lower cold crystallization temperature for PLA composites.

Higher degree of crystallinity for both PP and PLA composites were observed after the incorporation of the hybrid materials. As reported by other researchers previously, cellulose based materials could favor nucleation and crystal growth and thereby leading to the increase in the crystallinity of thermoplastic resins.

In terms of copper ion release after the immersion of composite films in an aqueous medium for a month, the preliminary results revealed that they were able to migrate only from PLA thin films. Copper ions were not able to leach from the extruded-injected PP or PLA composites. The copper ions release was detected for some PLA films, but the amount of copper ions leaching was insufficient to inactivate of E.coli. Therefore, no antimicrobial activity against E.coli DH5 $\alpha$ was demonstrated.

\subsection{References}

Allahvaisi, S. (2012). Polypropylene in the Industry of Food Packaging. INTECH Open Access Publisher.

AOAC (1995) Official methods of analysis. 16th ed. Association of Official Analytical Chemists International, Gaithersburg, MD. 
Appendini, P., \& Hotchkiss, J. H. (2002). Review of antimicrobial food packaging. Innovative Food Science \& Emerging Technologies, 3(2), 113-126.

ASTM (2010) D 638-10 Standard test method for tensile properties of plastics, West Conshohocken, PA.

Avila-Orta, C. A., Medellín-Rodríguez, F. J., Dávila-Rodríguez, M. V., Aguirre-Figueroa, Y. A., Yoon, K., \& Hsiao, B. S. (2007). Morphological features and melting behavior of nanocomposites based on isotactic polypropylene and multiwalled carbon nanotubes. Journal of Applied Polymer Science, 106(4), 2640-2647.

Bajpai, P. K., Singh, I., \& Madaan, J. (2012). Comparative studies of mechanical and morphological properties of polylactic acid and polypropylene based natural fiber composites. Journal of Reinforced Plastics and Composites, 31(24), 1712-1724.

Bikiaris, D. N., \& Triantafyllidis, K. S. (2013). HDPE/Cu-nanofiber nanocomposites with enhanced antibacterial and oxygen barrier properties appropriate for food packaging applications. Materials Letters, 93, 1-4.

Black, J. L., \& Jaczynski, J. (2006). Temperature effect on inactivation kinetics of Escherichia coli O157: H7 by electron beam in ground beef, chicken breast meat, and trout fillets. Journal of Food Science, 71(6), M221-M227.

Bahar, E., Ucar, N., Onen, A., Wang, Y., Oksüz, M., Ayaz, O., ... \& Demir, A. (2012). Thermal and mechanical properties of polypropylene nanocomposite materials reinforced with cellulose nano whiskers. Journal of Applied Polymer Science, 125(4), 2882-2889. 
Delgado, K., Quijada, R., Palma, R., \& Palza, H. (2011). Polypropylene with embedded copper metal or copper oxide nanoparticles as a novel plastic antimicrobial agent. Letters in Applied Microbiology, 53(1), 50-54.

Del Nobile, M. A., Cannarsi, M., Altieri, C., Sinigaglia, M., Favia, P., Iacoviello, G., \& d'Agostino, R. (2004). Effect of Ag-containing Nano-composite Active Packaging System on Survival of Alicyclobacillus acidoterrestris. Journal of Food Science, 69(8), E379-E383.

Duncan, T. V. (2011). Applications of nanotechnology in food packaging and food safety: barrier materials, antimicrobials and sensors. Journal of Colloid and Interface Science, 363(1), 1-24.

Echegoyen, Y., \& Nerín, C. (2013). Nanoparticle release from nano-silver antimicrobial food containers. Food and Chemical Toxicology, 62, 16-22.

Emamifar, A., Kadivar, M., Shahedi, M., \& Soleimanian-Zad, S. (2010). Evaluation of nanocomposite packaging containing $\mathrm{Ag}$ and $\mathrm{ZnO}$ on shelf life of fresh orange juice. Innovative Food Science \& Emerging Technologies, 11(4), 742-748.

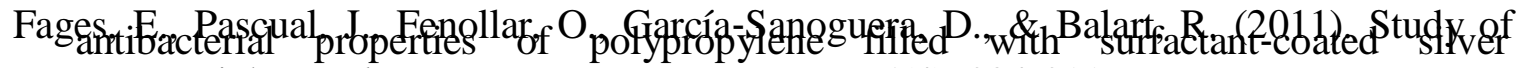
nanoparticles. Polymer Engineering \& Science, 51(4), 804-811.

Fan, Y., Zhang, C., Xue, Y., Zhang, X., Ji, X., \& Bo, S. (2011). Microstructure of two polypropylene homopolymers with improved impact properties. Polymer,52(2), 557563. 
Fernández, A., Soriano, E., Hernández-Muñoz, P., \& Gavara, R. (2010). Migration of antimicrobial silver from composites of polylactide with silver zeolites. Journal of Food Science, 75(3), E186-E193.

Fischer, E. W., Sterzel, H. J., \& Wegner, G. (1973). Investigation of the structure of solution grown crystals of lactide copolymers by means of chemical reactions. KolloidZeitschrift und Zeitschrift für Polymere, 251(11), 980-990.

Fortunati, E., Armentano, I., Iannoni, A., \& Kenny, J. M. (2010). Development and thermal behaviour of ternary PLA matrix composites. Polymer Degradation and Stability, 95(11), 2200-2206.

Fortunati, E., Armentano, I., Zhou, Q., Iannoni, A., Saino, E., Visai, L., ... \& Kenny, J. M. (2012a). Multifunctional bionanocomposite films of poly (lactic acid), cellulose nanocrystals and silver nanoparticles. Carbohydrate Polymers, 87(2), 1596-1605.

Fortunati, E., Armentano, I., Zhou, Q., Puglia, D., Terenzi, A., Berglund, L. A., \& Kenny, J. M. (2012b). Microstructure and nonisothermal cold crystallization of PLA composites based on silver nanoparticles and nanocrystalline cellulose. Polymer Degradation and Stability, 97(10), 2027-2036.

Fortunati, E., Peltzer, M., Armentano, I., Jiménez, A., \& Kenny, J. M. (2013). Combined effects of cellulose nanocrystals and silver nanoparticles on the barrier and migration properties of PLA nano-biocomposites. Journal of Food Engineering, 118(1), 117-124.

Frone, A. N., Berlioz, S., Chailan, J. F., \& Panaitescu, D. M. (2013). Morphology and thermal properties of PLA-cellulose nanofibers composites. Carbohydrate Polymers, 91(1), 377-384. 
Grigoriadou, I., Paraskevopoulos, K. M., Karavasili, M., Karagiannis, G., Vasileiou, A., \& Bikiaris, D. (2013). HDPE/Cu-nanofiber nanocomposites with enhanced mechanical and UV stability properties. Composites Part B: Engineering, 55, 407-420.

Hong, S. I., \& Krochta, J. M. (2006). Oxygen barrier performance of whey-protein-coated plastic films as affected by temperature, relative humidity, base film and protein type. Journal of Food Engineering, 77(3), 739-745.

Isogai, A., Saito, T., \& Fukuzumi, H. (2011). TEMPO-oxidized cellulose nanofibers. Nanoscale, 3(1), 71-85.

Jiang, J., Li, G., Ding, Q., \& Mai, K. (2012). Ultraviolet resistance and antimicrobial properties of $\mathrm{ZnO}$-supported zeolite filled isotactic polypropylene composites. Polymer Degradation and Stability, 97(6), 833-838.

Jokar, M., Rahman, R. A., Ibrahim, N. A., Abdullah, L. C., \& Tan, C. P. (2012). Melt production and antimicrobial efficiency of low-density polyethylene (LDPE)-silver nanocomposite film. Food and Bioprocess Technology, 5(2), 719-728.

Jonoobi, M., Harun, J., Mathew, A. P., \& Oksman, K. (2010). Mechanical properties of cellulose nanofiber (CNF) reinforced polylactic acid (PLA) prepared by twin screw extrusion. Composites Science and Technology, 70(12), 1742-1747.

Kale, G., Auras, R., \& Singh, S. P. (2006). Degradation of commercial biodegradable packages under real composting and ambient exposure conditions. Journal of Polymers and the Environment, 14(3), 317-334.

Kazmiruk, V. (2012). Scanning Electron Microscopy. InTech, New York. 737 pp. 
Levanduski, L., \& Jaczynski, J. (2008). Increased resistance of Escherichia coli O157: H7 to electron beam following repetitive irradiation at sub-lethal doses. International Journal of Food Microbiology, 121(3), 328-334.

Lezak, E., Kulinski, Z., Masirek, R., Piorkowska, E., Pracella, M., \& Gadzinowska, K. (2008). Mechanical and thermal properties of green polylactide composites with natural fillers. Macromolecular Bioscience, 8(12), 1190-1200.

Liu, Y., \& Donovan, J. A. (1995). Miscibility and crystallization of semicrystalline nylon 6 and amorphous nylon 6IcoT blends. Polymer, 36(25), 4797-4803.

Li, X., Xing, Y., Jiang, Y., Ding, Y., \& Li, W. (2009). Antimicrobial activities of ZnO powder-coated PVC film to inactivate food pathogens. International Journal of Food Science \& Technology, 44(11), 2161-2168.

Masirek, R., Kulinski, Z., Chionna, D., Piorkowska, E., \& Pracella, M. (2007). Composites of poly (L-lactide) with hemp fibers: Morphology and thermal and mechanical properties. Journal of Applied Polymer Science, 105(1), 255-268.

Mathew, A. P., Oksman, K., \& Sain, M. (2006). The effect of morphology and chemical characteristics of cellulose reinforcements on the crystallinity of polylactic acid. Journal of Applied Polymer Sscience, 101(1), 300-310.

Nyambo, C., Mohanty, A. K., \& Misra, M. (2010). Polylactide-based renewable green composites from agricultural residues and their hybrids. Biomacromolecules, 11(6), 1654-1660.

Oksman, K., Skrifvars, M., \& Selin, J. F. (2003). Natural fibres as reinforcement in polylactic acid (PLA) composites. Composites Science and Technology, 63(9), 13171324. 
Paleo, A. J., Sencadas, V., Hattum, F. W. J., Lanceros-Méndez, S., \& Ares, A. (2014). Carbon nanofiber type and content dependence of the physical properties of carbon nanofiber reinforced polypropylene composites. Polymer Engineering \& Science, 54(1), 117-128.

Pluta, M., Galeski, A., Alexandre, M., Paul, M. A., \& Dubois, P. (2002). Polylactide/montmorillonite nanocomposites and microcomposites prepared by melt blending: structure and some physical properties. Journal of Applied Polymer Science, 86(6), 1497-1506.

Saito, T., Kimura, S., Nishiyama, Y., \& Isogai, A. (2007). Cellulose nanofibers prepared by TEMPO-mediated oxidation of native cellulose. Biomacromolecules, 8(8), 2485-2491.

Srithep, Y., Ellingham, T., Peng, J., Sabo, R., Clemons, C., Turng, L. S., \& Pilla, S. (2013). Melt compounding of poly (3-hydroxybutyrate-co-3-hydroxyvalerate)/nanofibrillated cellulose nanocomposites. Polymer Degradation and Stability, 98(8), 1439-1449.

Tsuji, H., \& Ikada, Y. (1996). Blends of isotactic and atactic poly (lactide) s: 2. Molecularweight effects of atactic component on crystallization and morphology of equimolar blends from the melt. Polymer, 37(4), 595-602.

Wu, J. J., Lee, G. J., Chen, Y. S., \& Hu, T. L. (2012). The synthesis of nanosilver/polypropylene plastics for antibacterial application. Current Applied Physics, 12, S89-S95.

Yasuniwa, M., Sakamo, K., Ono, Y., \& Kawahara, W. (2008). Melting behavior of poly (1lactic acid): X-ray and DSC analyses of the melting process. Polymer, 49(7), 19431951. 
Zhang, W., Zhang, Y., Ji, J., Yan, Q., Huang, A., \& Chu, P. K. (2007). Antimicrobial polyethylene with controlled copper release. Journal of Biomedical Materials Research Part A, 83(3), 838-844.

Zhong, T., Oporto, G. S., Jaczynski, J., Tesfai, A. T., \& Armstrong, J. (2013). Antimicrobial properties of the hybrid copper nanoparticles-carboxymethyl cellulose. Wood and Fiber Science, 45(2), 215-222. 


\section{CHAPTER 6: DRYING CELLULOSE-BASED MATERIALS CONTAINING COPPER NANOPARTICLES}

\subsection{Chapter abstract}

Carboxymethyl cellulose and TEMPO nanofibrillated cellulose were used as substrates to synthesize copper nanoparticles from copper sulfate and they were subsequently dried using spray-, freeze- and modified freeze drying processes. Morphological characterization, particle size distribution, copper quantification and the oxidation state of copper were evaluated. The morphology and the size distribution of the dried particles were characterized using scanning electron microscopy-energy dispersive X-ray spectroscopy; particle size distribution was evaluated using laser diffraction; copper content was determined by inductively coupled plasma-optical emission spectroscopy; and finally, the oxidation state of copper was determined using X-ray photoelectron spectroscopy and Xray diffraction. Plate-like structures of cellulose and cellulose-copper nanoparticles were formed after the freeze-drying process, with length and width over hundreds of microns. Most of the spray dried particles exhibited spherical shapes with the particle size ranging from around $300 \mathrm{~nm}$ to several microns. The spray drying process caused more copper loss compared with freeze drying, most probably because of the additional steps required to perform the spray drying. XPS results showed that the state of oxidation of the final dried material depends on the specific substrate. CMC surfaces exhibit mostly $\mathrm{Cu}^{0}$ and/or $\mathrm{Cu}^{+}$. On the contrary TNFC surfaces present mostly $\mathrm{Cu}^{2+}$. Solvent exchange process using ethanol and butanol creates more porous structures on the CMC substrates. A solvent exchange process using ethanol and tert-butanol sequence facilitates the formation of a more porous structure on TEMPO nanofibrillated cellulose. 


\subsection{Introduction}

Properties of nanocellulose such as high strength, low weight, high aspect ratio, high specific surface area, biodegradability, renewability, and feasibility to be chemically modified have provided opportunities for applications in pharmaceutical, bio-medical, cosmetic, electronics, packaging, catalysis, safety and environmental areas (Eichhorn et al. 2010, Klemm et al. 2011). Nanocellulose (which includes cellulose nanofibers and cellulose nanocrystals) is produced in aqueous suspensions and even though there are applications that do not require a drying process to use them (e.g., papermaking), there are many others that demand the material to be dried. In our previous research we developed a facile method to incorporate copper nanoparticles in suspensions of cellulose based materials, including nanocellulose; the final hybrid materials resulting in effective antimicrobial properties after a solvent casting process (Zhong et al. 2013). Considering that our long-term research goal is to incorporate this hybrid material in thermoplastic resins, our current challenge and the main goal proposed in this research is to find an adequate process to dry it without modifying the copper state of oxidation and producing minimum agglomeration between the cellulosic components.

Hydrogen bonding and Van der Waals forces among cellulose are formed to hold them together and are the main cause of particle aggregation during the removal of water in the cellulosic drying processes (Gardner et al. 2008). To date, several studies have been performed with the aim of drying and characterizing cellulose nanostructures, however much work is still necessary to minimize those cellulosic aggregations and maintain the properties of the original nanostructures. According to Peng et al. (2013a, b), for example, drying nanocellulose will affect, in addition their crystallinity, thermostability and surface energies.

Spray drying and freeze drying processes have been the main processes used to dry nanocellulose (Pakowski et al. 2007; Elanthikkal et al. 2010; Peng et al. 2012a). 
The basic idea of spray drying is the production of highly dispersed powders from a fluid feed by evaporating the solvent. This is achieved by mixing a heated gas with an atomized (sprayed) fluid of high surface-to-mass ratio droplets within a vessel (drying chamber), causing the solvent to evaporate uniformly and quickly through direct contact of the gas. During the spray drying process, both at laboratory and pilot scale, better control of the particle size (uniformity) and morphology have been reported (Nandiyanto and Okuyama 2011). Elanthikkal et al. (2010) used spray drying to dry cellulose microfibers and obtained "doughnut shape" particles with diameters between $2 \mu \mathrm{m}$ and $15 \mu \mathrm{m}$. Peng et al. (2012a) used spray drying to dry cellulose nanocrystals (CNC) and nanofibrillated cellulose (NFC); they reported that spray drying of CNC suspension resulted in ninety percent of the dried particles with the size below $6.76 \mu \mathrm{m}$, and spray drying of NFC suspension led to the production of ninety percent of dried particles with the size below $7.48 \mu \mathrm{m}$. The authors suggested that spray drying might a suitable way to dry CNC and NFC suspension for their potential application as reinforcement in thermoplastic resins. One of the most significant difficulties associated with the spray drying process is the high temperature required in the process which might be inadequate for heat-sensitive products.

Freeze drying has been considered effective for drying heat sensitive products. A freezedrying process consists of removing water from a frozen sample by sublimation and desorption under vacuum. Three main steps considered in a freeze-drying process are: freezing, first drying and secondary drying (Abdelwahed et al. 2006). During the first step the liquid suspension is cooled, and ice crystals of pure water are formed. In the second step the ice sublimes and the water vapor condenses on the condenser. At the end of the sublimation step a resulting porous material is expected because of the spaces that were occupied by ice crystals. In the secondary drying a removal of the absorbed water from the product is expected. During the freezing and dehydration many stresses might be generated that can destabilize the original colloidal suspension inducing undesirable aggregations. Jin et al. (2004) and Peng et al. (2012a) also reported that directly freeze drying cellulosic materials from water caused the lateral aggregation and the collapsed network structures of cellulose because of capillary effects, diffusion forces and hydrogen bonding formation. 
In addition, a modified freeze drying process that considers a preliminary solvent exchange step has been proposed. The main goal here is to preserve the morphology and surface area of the nanocellulosic materials (Jin et al. 2004; Sehaqui et al. 2011a-b). The solvent corresponding to a less hydrophilic liquid (typically an alcohol) reduces the capillary effects during the drying process increasing the porosity and surface area (Sehaqui et al. 2011b).

In this study the effect of a spray drying, a freeze drying and two solvent exchange pretreatments previous to a freeze drying process were evaluated on hybrids of cellulosebased materials and copper nanoparticles. The final evaluation included morphological analysis, particle size and distribution, crystallinity and oxidation state of copper of the hybrid materials before and after the drying processes.

\subsection{Materials and methods}

\subsubsection{Materials}

Sodium carboxymethyl cellulose (CMC) (average molecular weight: 90,000) from Sigma Aldrich, USA.; technical-crystal cupric sulfate pentahydrate $\left(\mathrm{CuSO}_{4} \cdot 5 \mathrm{H}_{2} \mathrm{O}\right)$ from fisher Scientific, USA; sodium borohydride $\left(\mathrm{NaBH}_{4}\right)(0.5 \mathrm{M})$ from Acros Organics, USA.; ethanol (reagent alcohol, specially denatured alcohol formula 3A 95\% and isopropyl alcohol 5\% by volume) from Sigma Aldrich, USA.; nitric acid (69\%-71\% w/w) from Fisher Scientific, USA.; TEMPO nanofibrillated cellulose (TNFC) (0.67 wt. \%) from the Forest Product Laboratory, Madison, WI; butanol (Certified ACS) from Fisher Scientific, USA; tert-butanol (99.5\%, for analysis) from Acros Organic, USA; sodium carboxymethyl cellulose, sodium borohydride, ethanol, butanol and tert-butanol were used as received. 


\subsubsection{Preparation of control suspensions of cellulose base materials: CMC and TNFC}

One gram of CMC was dissolved in $49 \mathrm{~mL}$ of deionized water under vigorous magnetic stirring. The resulting suspension of approximately $50 \mathrm{~mL}$ of CMC suspension (2 wt.\%) was used as the control. TEMPO nanofibrillated cellulose $(0.67$ wt. $\%)$ from Forest Product Laboratory was used as control TNFC suspension.

\subsubsection{Preparation of the hybrid CMC-copper nanoparticles}

The general procedure used for the preparation of the hybrid carboxymethyl cellulosecopper nanoparticles was described previously (Zhong et al. 2013). Ten milliliters of cupric sulfate solution $(0.1 \mathrm{~mol} / \mathrm{L})$ were added by drops into the CMC control suspension. The mixtures of $\mathrm{CMC}$ and cupric sulfate were subjected to high-speed mixing while the cupric sulfate solution was added. Then the mixture was allowed to react at room temperature for $3 \mathrm{~h}$. After that, cupric ions were reduced to metallic copper or copper oxide nanoparticles by adding, while stirring, $4 \mathrm{~mL}$ of reducing agent sodium borohydride. The resulting hybrid material was coded as CMC-copper nanoparticles, standing for CMC containing copper nanoparticles.

\subsubsection{Preparation of the hybrid TNFC-copper nanoparticles}

A $2.4-\mathrm{mL}$ aliquot of cupric sulfate solution $(0.1 \mathrm{~mol} / \mathrm{L})$ was added by drops into thirty six grams of TNFC gel (0.67 wt.\%). The mixture of TNFC and cupric sulfate was subjected to high-speed mixing while the cupric sulfate solution was added. Then the mixture was allowed to react at room temperature for $3 \mathrm{~h}$. After that, cupric ions were reduced to metallic copper or copper oxide nanoparticles by adding, while stirring, $0.48 \mathrm{~mL}$ of reducing agent sodium borohydride. 


\subsubsection{Spray drying process}

The raw materials used for the spray drying process corresponded to suspensions of CMC (2 wt.\%), CMC-copper nanoparticles (2 wt.\%) and TNFC (0.335 wt.\%). Spray drying was performed at the University of Maine, using a laboratory-scale Mini Spray Dryer B-290 (Buchi, Switzerland). The parameters used corresponded to: inlet temperature: $180{ }^{\circ} \mathrm{C}$; aspirator: $100 \%$ (drying gas flow rate was approx. $35 \mathrm{~m}^{3} / \mathrm{h}$ ); suspension feed rate: $20 \%$ (6 $\mathrm{mL} / \mathrm{min})$; gas feed rate: $473 \mathrm{~L} / \mathrm{h}$; outlet temperatures for the three samples: CMC $\left(85^{\circ}{ }^{\circ} \mathrm{C}\right)$, CMC-copper nanoparticles $\left(99^{\circ} \mathrm{C}\right)$ and TNFC $\left(89^{\circ} \mathrm{C}\right)$.

\subsubsection{Freeze drying process}

The raw materials used for the freeze drying process corresponded to suspensions of CMC (2 wt.\%), CMC-copper nanoparticles (2 wt.\%), TNFC (0.335 wt.\%) and TNFC-copper nanoparticles (0.335 wt.\%). Freeze drying was conducted on a VirTis Genesis Freeze Dryer (Warminster, PA, USA) at $-40{ }^{\circ} \mathrm{C}$ for 1 week.

\subsubsection{Modified freeze drying process}

Before the freeze drying process, the raw materials were exposed to the following solvent exchange pretreatments: 1: ethanol followed by 1-butanol; 2: ethanol followed by tertbutanol.

In Table 6.1 the drying experiments and raw materials used in this research are summarized. Spray drying was not performed on TNFC-copper nanoparticles. (see discussion). 
Table 6. 1 Drying experiments.

\begin{tabular}{ccccc}
\hline Drying Process & CMC & $\begin{array}{c}\text { CMC-copper } \\
\text { nanoparticles }\end{array}$ & TNFC & TNFC-copper nanoparticles \\
\hline Spray (SD) & $\mathrm{X}$ & $\mathrm{X}$ & $\mathrm{X}$ & --- \\
Freeze (FD) & $\mathrm{X}$ & $\mathrm{X}$ & $\mathrm{X}$ & $\mathrm{X}$ \\
Modified freeze & $\mathrm{X}$ & $\mathrm{X}$ & $\mathrm{X}$ & $\mathrm{X}$ \\
$\begin{array}{c}\text { freeze } 1^{*}(\mathrm{E} / 1-\mathrm{B}-\mathrm{BD}) \\
\text { Modified freeze } 2^{* *} \\
\text { (E/tert-B-FD) }\end{array}$ & $\mathrm{X}$ & $\mathrm{X}$ & $\mathrm{X}$ & $\mathrm{X}$ \\
\hline
\end{tabular}

*Ethanol/1-butanol;

** Ethanol/tert-butanol

\subsubsection{Field emission scanning electron microscopy-energy dispersive $X$-ray spectroscopy characterization (FESEM-EDX)}

The morphological and copper elemental quantification analyses of the spray-, freeze- and modified freeze-dried materials were characterized using a Hitachi S-4700 FESEM with an EDAX brand energy dispersive spectroscopy detector (Tokyo, Japan). Before the FESEMEDX analyses, all the samples were coated with gold-palladium using a sputtering process.

\subsubsection{Laser diffraction (LD) characterization}

Spray dried samples were dispersed in ethanol for particle size distribution measurement. They were mixed by stirring using a magnetic bar followed by ultrasonic treatment for 10 min. Particle size distribution in each suspension at $0.5 \mathrm{wt} . \%$ was measured with a Malvern Mastersizer 2000 (size range from $0.2 \mu \mathrm{m}-2000 \mu \mathrm{m}$ ). Measurements were performed five times. Average diameter of each sample at 10,50 and 90 percentile, and standard deviations were obtained.

\subsubsection{Inductively coupled plasma-optical emission spectroscopy (ICP-OES) characterization}

Copper content present in the hybrid CMC-copper nanoparticles and TNFC-copper nanoparticles suspensions and in the correspondent dried materials (spray-, freeze- and 
modified freeze-dried) were determined using a Varian Vista-PRO CCD simultaneous ICPOES (Palo Alto, USA). Five drops of concentrated nitric acid were added into the hybrid suspensions to digest metallic copper or copper oxide nanoparticles and then they were analyzed by ICP-OES. The experiments and analyses were performed in duplicate. For the determination of copper content in the dried materials, $100 \mathrm{mg}$ of each dried material was digested using $4 \mathrm{~mL}$ of nitric acid, and then the mixture was transferred into a volumetric flask (100 mL); deionized water was added to complete the $100-\mathrm{mL}$ scale line. The experiments and analyses were performed in duplicate.

\subsubsection{X-ray photoelectron spectroscopy (XPS) characterization}

The oxidation states of the copper in the spray-dried (SD), freeze-dried (FD) and modified freeze-dried materials (E/1-B-FD CMC based material, E/tert-B-FD TNFC based material) were determined using a Physical Electronics VersaProbe 5000 X-ray Photoelectron Spectroscope with a monochromatic Aluminum Ka X-ray source (Chanhassen, MI, USA). The base pressure in the high vacuum analysis chamber was around $2 \times 10^{-6} \mathrm{~Pa}$. An Aluminum X-ray source of $1486.6 \mathrm{eV}$ was used for photoelectron excitation with X-ray power of $25 \mathrm{~W}$ and anode voltage of $15 \mathrm{kV}$. Pass energies of $117.4 \mathrm{eV}$ for survey scan and $23.5 \mathrm{eV}$ for detail scan were used for the data acquisition with energy steps of $0.5 \mathrm{eV}$ for survey scan and $0.05 \mathrm{eV}$ for detail scan, respectively. PHI MultiPak software was used for element identification and peak fitting. The C1s peak at a binding energy of $284.8 \mathrm{eV}$ was used as the internal reference. A Shirley-type background was subtracted from the spectra and Gauss-Lorentz curves were used to fit the spectra.

\subsubsection{X-ray diffraction (XRD) characterization}

The copper crystal structures of the spray-dried (SD), freeze-dried (FD) and modified freeze-dried materials (E/1-B-FD CMC based materials, E/tert-B-FD TNFC based materials) was studied using an X-ray diffractometer (PANalytical X'Pert Pro, Almelo, Netherlands) with a $\mathrm{Cu} \mathrm{K \alpha} \mathrm{X}$-ray source. The crystallite size of copper on each sample was 
calculated in duplicate using the Scherrer equation (Eq.1) (Djurado et al. 2000; Borchert et al. 2005; Liu et al. 2006).

$D=\frac{K \lambda}{\beta \cos \theta}$

Where $D$ is the average of crystallite size; $K$ is the Scherrer constant $(0.94$ for cubic crystallites like $\mathrm{Cu}_{2} \mathrm{O}$ (Borchert et al. 2005)); $\lambda$ is the $\mathrm{X}$-ray wavelength $(0.15418 \mathrm{~nm}$ for this study); ${ }^{\beta}$ is full width at half-maximum (FWHM) of the diffraction peak; ${ }^{\theta}$ is Bragg angle.

\subsection{Results and discussion}

\subsubsection{Morphology of the spray-, freeze- and modified freeze dried cellulosic materials}

Electronic microscopy analysis shows clear differences among the three drying processes investigated. Figure 6.1 presents SEM images of the spray-dried materials (left side pictures) and the freeze-dried materials (right side pictures). All spray-dried materials have spherical shapes with external voids and craters; on the other hand, all freeze-dried materials present a plate-like shape attributed to lateral aggregation of the cellulose nanofibrils. Similar results have been presented previously by Peng et al. (2012b) when drying cellulose nanocrystals and nanofibrillated cellulose materials.

Spray-dried materials presented different size distributions depending on the original raw material. Using the Image J software (Ferreira and Rasband 2012) and analyzing more than fifty particles in each of the spray-dried images we determined that the average diameter of the CMC particles was $5.8 \mu \mathrm{m}$ with a standard deviation of $4.3 \mu \mathrm{m}$ (Figure 6.1a). (about $500 \mathrm{~nm}$ to $10 \mu \mathrm{m})$. Smaller spherical particles with diameters less than $1 \mu \mathrm{m}$ were deposited inside the "mushroom cap-shape" CMC particles as presented in Figure 6.1a (inset, higher magnification picture). Similar morphology, but larger and less uniform 
particles were found for spray-dried CMC-copper nanoparticles (Figure 6.1c). The average diameter in this case was $11.4 \mu \mathrm{m}$ with a standard deviation of $7.1 \mu \mathrm{m}$. The higher level of agglomeration might be caused by the attachment of copper nanoparticles on the CMC surfaces. This finding requires further investigation.

As expected, and as presented in Figure 6.1e the TEMPO nanofibrillated cellulose (TNFC) spray- dried material resulted in smaller sizes and narrower size distribution compared with the $\mathrm{CMC}$ particles and CMC-copper nanoparticles. The average diameter was $3.8 \mu \mathrm{m}$ with a standard deviation of $2.1 \mu \mathrm{m}$. Peng et al. (2012a, 2012b) have demonstrated that smaller size and narrower size distribution of original cellulose in aqueous suspensions led to the production of smaller particles after the spray drying.

In general, for all freeze-dried material, the length and width of the plate-like materials were over hundreds of microns (Figure 6.1b, 6.1d, 6.1f). The phenomenon of lateral aggregation of cellulose during freeze drying was also reported by Hult et al. (2001) and Peng et al. (2012a). Cellulose nanofibrils are aligned laterally via hydrogen bonding to form a single layer; each single layer is bonded together via hydrogen bonding. The hydrogen bonding between each layer is weaker than the lateral hydrogen bonding within the layer and therefore easier to break (Peng et al. 2012a). In Figure 6.2 pictures of four freeze-dried materials (left side) and the same materials, but pre-treated using ethanol/1butanol solvent exchange before the freeze drying process (right side pictures) are presented All materials displayed an increase in their apparent porosity after the pretreatment, and the resulting pores were larger for $\mathrm{CMC}$ and $\mathrm{CMC}-\mathrm{Cu}$ materials (Figure $6.2 \mathrm{~b}$ and $6.2 \mathrm{~d}$ ) compared with those from TNFC (Figure 6.2f and 6.2h). In Figure 6.3 the effect of ethanol/tert-butanol solvent exchange pretreatment before the freeze drying process for both CMC and TNFC based materials are displayed. No significant effect of ethanol/tert-butanol solvent exchange process on the morphology of CMC based materials, thin and large plate-like structures are shown in Figure 6.3b and 6.3d. 

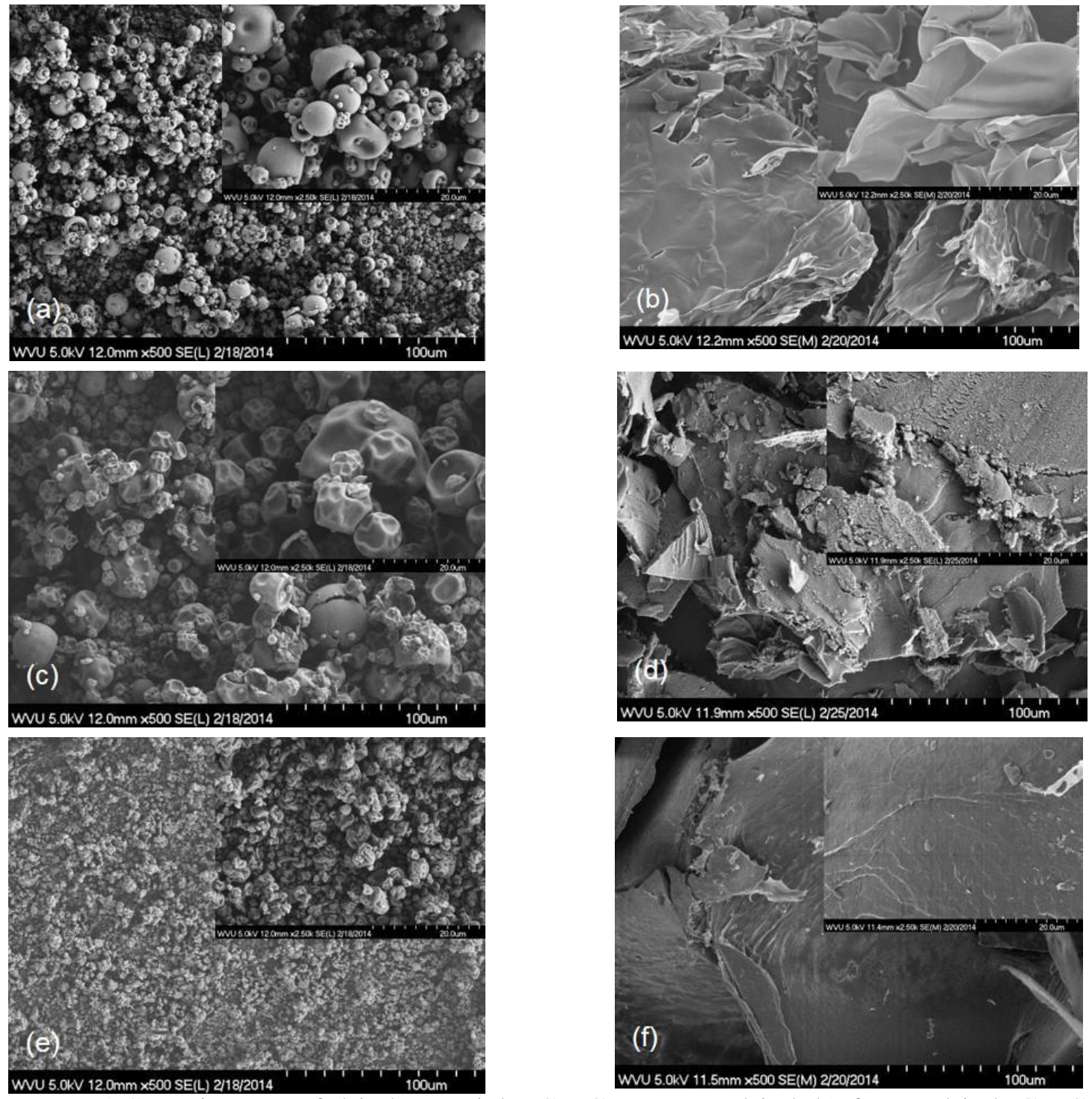

Figure 6. 1 SEM images of dried materials. CMC: a) spray dried, b) freeze dried; CMCcopper nanoparticles: c) spray dried, d) freeze dried; TNFC: e) spray dried, f) freeze dried. 

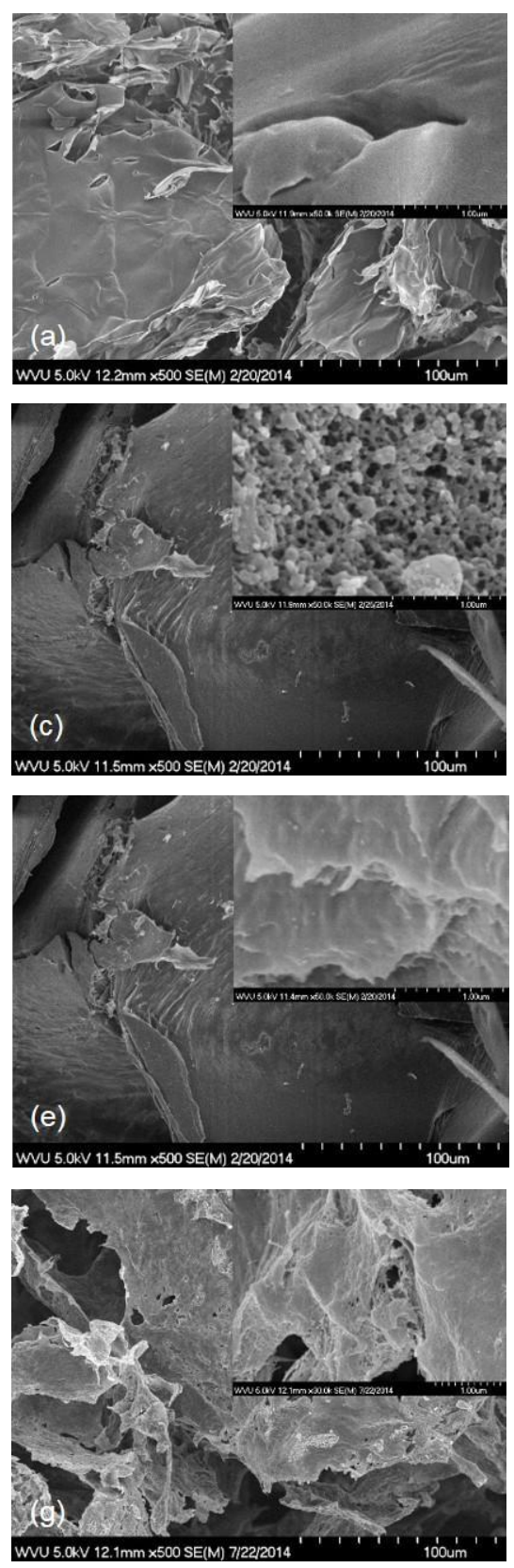
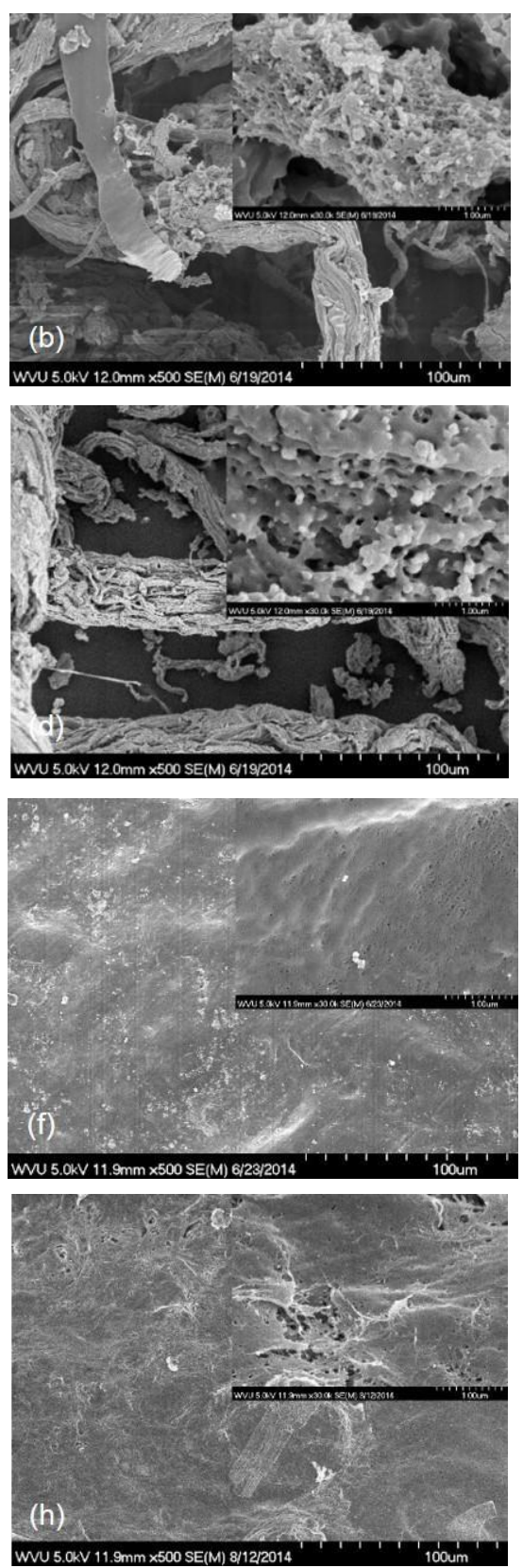

Figure 6. 2 SEM images of freeze and modified freeze dried materials: CMC: (a) CMC freeze dried, (b) $\mathrm{CMC}$ after an E/1-B-FD treatment; $\mathrm{CMC}-\mathrm{Cu}$ : (c) $\mathrm{CMC}-\mathrm{Cu}$ freeze dried, (d) CMC-Cu after an E/1-B-FD treatment; TNFC: (e) TNFC freeze dried, (f) TNFC after an E/1-B-FD treatment; TNFC-Cu: (g) TNFC-Cu freeze dried, (h) TNFC-Cu after an E/1B-FD treatment. 

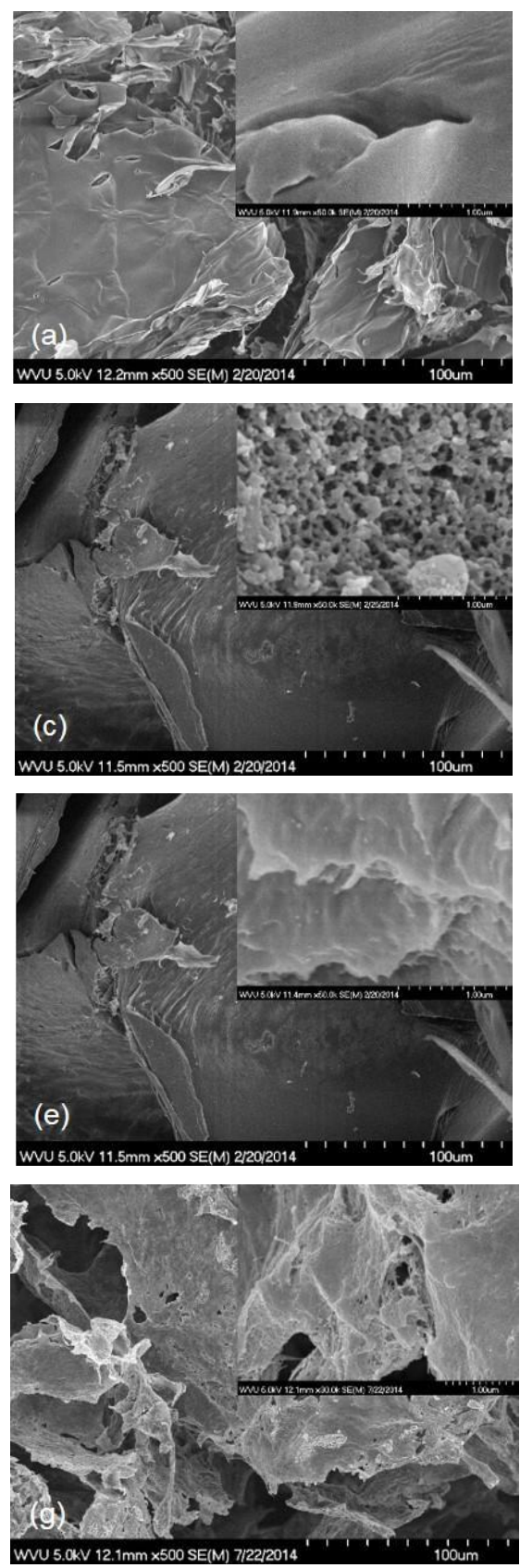
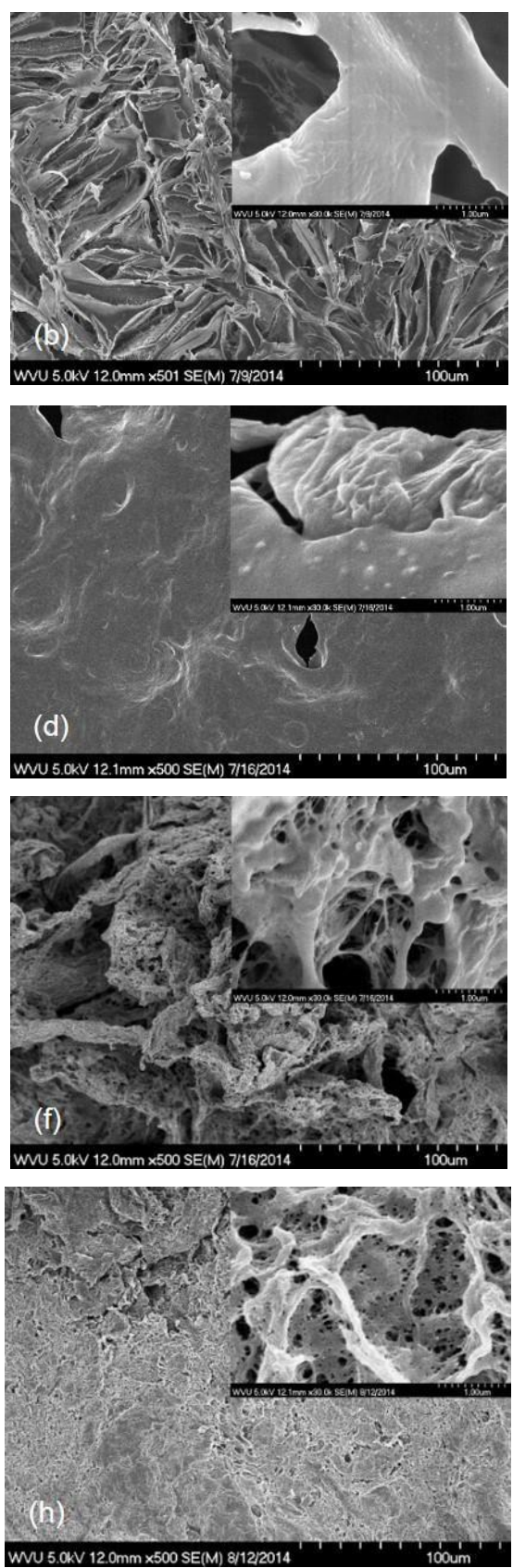

Figure 6. 3 SEM images of freeze and modified freeze dried materials: CMC: (a) CMC freeze dried, (b) CMC after an E/tert-B-FD treatment; CMC-Cu: (c) CMC freeze dried, (d) CMC-Cu after an E/tert-B-FD treatment; TNFC: (e) TNFC freeze dried, (f) TNFC after an E/tert-B-FD treatment; TNFC-Cu: (g) TNFC-Cu freeze dried, (h) TNFC-Cu after an E/tertB-FD treatment. 
However, a more important effect on the morphology of TNFC and TNFC-copper nanoparticles materials is produced with this pre-treatment before the freeze dried process (Figure 6.3f, 6.3h). A TNFC material with a highly porous structure and fibrous network is observed in both inset images of Figure 6.3f, 6.3h. The replacement of water by less hydrophilic liquids, such as alcohols, has partially reduced the agglomeration during the freeze-drying process of cellulose based materials and has improved their apparent porosity. In general, low surface tension alcohols (such as ethanol: $22.1 \mathrm{mN} / \mathrm{m}$, butanol: $24.2 \mathrm{mN} / \mathrm{m}$, and tert-butanol: $20.7 \mathrm{mN} / \mathrm{m}$ at $25^{\circ} \mathrm{C}$ ), will reduce capillary effects during the drying process and thus reduce agglomeration of cellulose materials (Sehaqui et al. 2011b). According to the results presented in Figure 6.2 and Figure 6.3, the use of 1-butanol (linear chain) or tert-butanol affects the morphology of the treated material differently. For both alcohols, the strongest intermolecular force is hydrogen bonding, however, because of its specific configuration, tert-butyl group will do a better job of blocking one side of the -OH group, making it a little bit harder to hydrogen bond. Compared to 1-butanol, the interaction of tert-butanol molecules with the cellulose will be different because of its spherical conformation which provides for a smaller volume to surface area ratio. The long straight chain of the 1-butanol molecule has much more surface area to interact with for the same volume. Dispersion forces between CMC and 1-butanol appear to be higher than those between TNFC and 1-butanol, and that might explain the higher apparent porosity for those dried CMC materials. On the contrary, the higher porosity presented by those TNFC after their pre-treatment with tert-butanol might be mostly attributed to steric effects of the tert-butyl alcohol structural conformation.

\subsubsection{Particle size distribution using laser diffraction (LD)}

Freeze-dried materials resulted in large aggregates of molecules resulting unpractical to measure their particle size. For spray-dried materials the results of particle size distribution and the average of particle size, measured by laser diffraction, are presented in Figure 6.4 and Figure 6.5, respectively. These figures show that the averages in diameter for spraydried $\mathrm{CMC}$ samples at 10,50 and 90 percentile are $0.32 \mu \mathrm{m}, 4.50 \mu \mathrm{m}$ and $10.63 \mu \mathrm{m}$, 
respectively. The averages in diameter for CMC-copper nanoparticles are significantly bigger resulting in $4.25 \mu \mathrm{m}, 11.64 \mu \mathrm{m}$ and $25.07 \mu \mathrm{m}$ at 10,50 and 90 percentile. The average particle size of the spray-dried CMC-copper nanoparticles at 90 percentile increased up to $135.8 \%$ compared to the original raw material CMC.
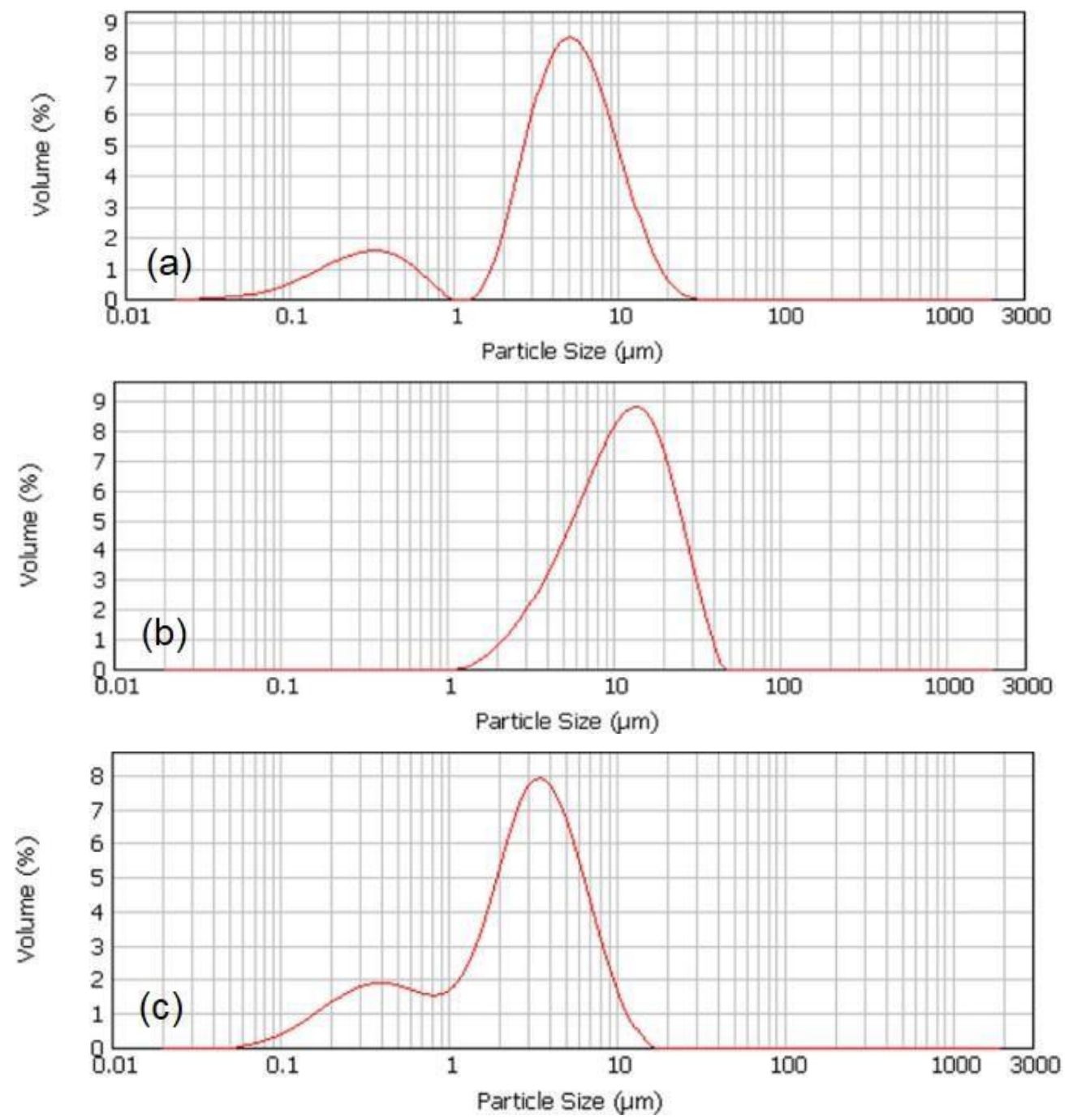

Figure 6. 4 Particle size distribution of spray dried particles: (a) CMC, (b) CMC-copper nanoparticles, (c) TNFC.

Apparently, copper is promoting the hydrogen bondability between cellulose molecules increasing the agglomeration of cellulose particles. As expected, spray-dried TEMPO nanofibrillated cellulose (TNFC) has a much smaller size and narrower size distribution 
compared with spray-dried CMC and CMC-copper nanoparticles; ninety percent of TNFC is below $6.69 \mu \mathrm{m}$ in diameter, $50 \%$ is $2.7 \mu \mathrm{m}$ and only $10 \%$ is $0.31 \mu \mathrm{m}$. The particle size of only a small portion of spray-dried TNFC materials has been reduced to nano-scale range (around three hundred nanometers). So, even the spray drying process generates a more uniform particle size, still cellulosic agglomerations generate a particle size that is more in the micrometer than in the nanometer range.

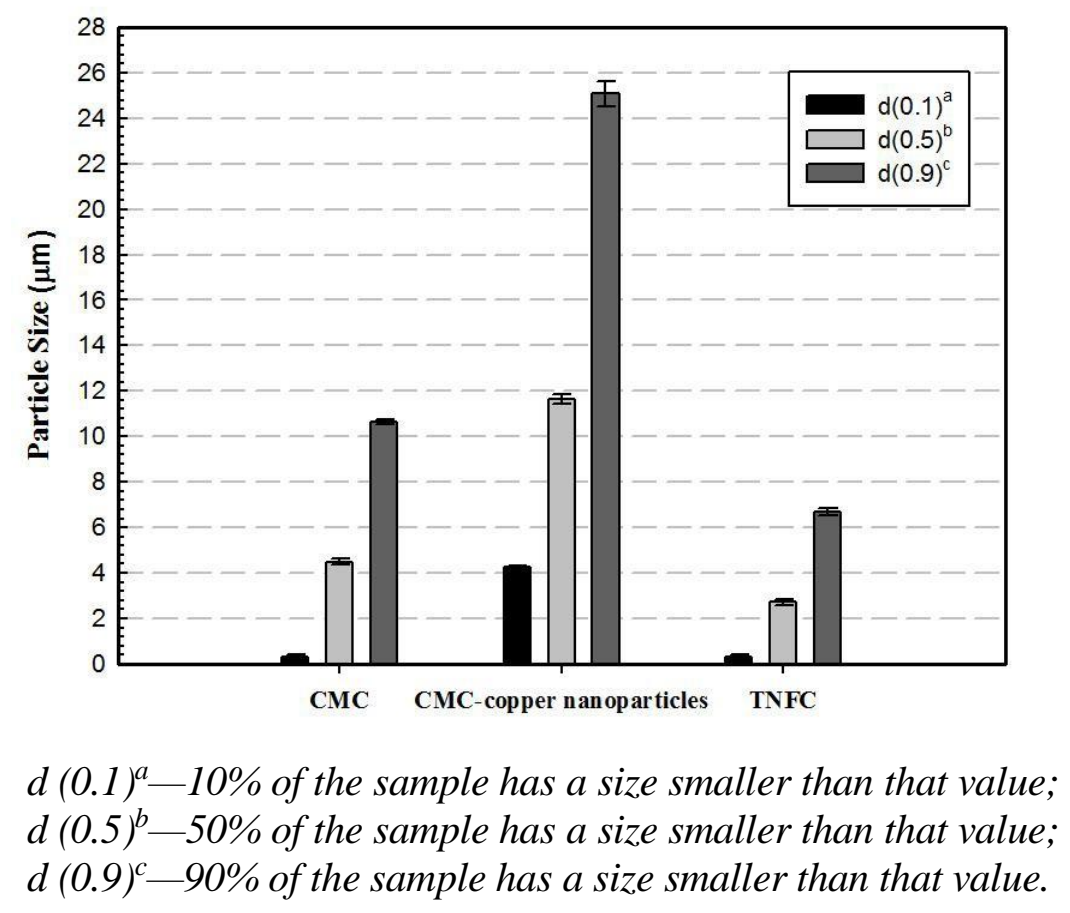

Figure 6. 5 Average diameter size histograms at 10, 50 and 90 percentile for various spray dried particles.

\subsubsection{Energy dispersive X-ray (EDX) elemental quantification and ICP-OES analysis to determine copper content on the dried materials}

To quantify copper content CMC-copper nanoparticles and TNFC-copper nanoparticles were exposed to energy dispersive X-ray (EDX) analysis. However, samples of TNFC- 
copper nanoparticles were extremely delicate and they were damaged during the exposure to the electron beam. Therefore, only data for the CMC-copper nanoparticles are available.
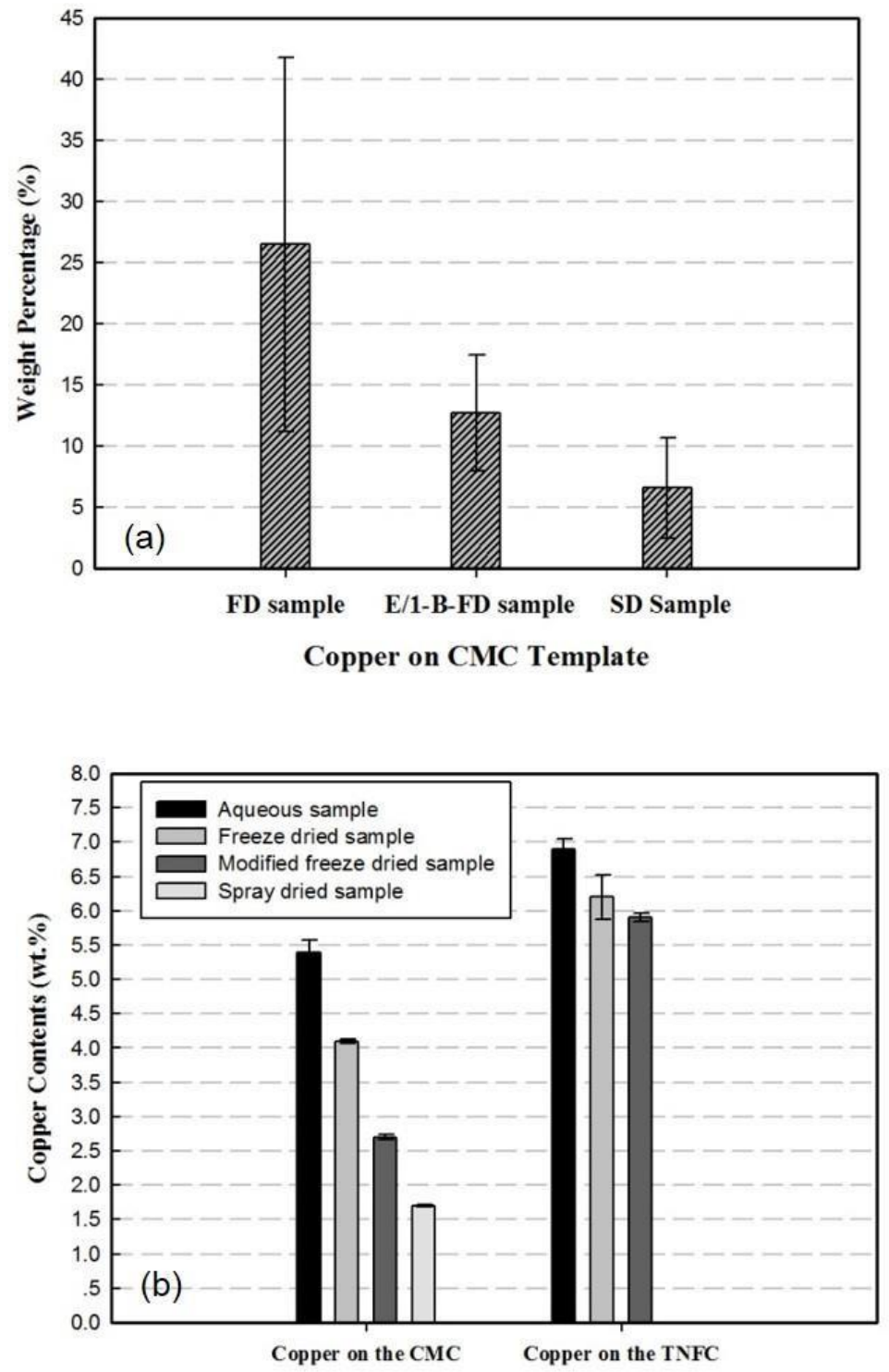

Copper on CMC and TNFC templates

Figure 6. 6 (a) Energy dispersive X-Ray (EDX) copper elemental quantification of dried CMC-copper nanoparticles materials, (b) ICP-OES quantification of copper contents on CMC and TNFC templates. 
In Figure 6.6a the results for copper elemental quantification on dried CMC-copper nanoparticles using EDX are presented. Even though an apparent reduction in the copper content depending on the drying method utilized is observed, the standard deviation analysis indicated that there is not significant differences in the copper content among samples $(p$-value $=0.097>0.05$ ). This high standard deviation might be attributed to the heterogeneous distribution of copper on the samples or some EDX parameters that are interfering in the analysis. EDX analysis is considered a semi-quantitative analysis since some analytical variables, such as critical penetration depth and detection limit might affect the results. Particle size, surface irregularity and moisture content on the samples might also affect the variability of the results (Markowicz 2011).

Inductively coupled plasma-optical emission spectrometry (ICP-OES) was the second method used to determine copper content in solid and liquid samples containing CMC- and TNFC-copper nanoparticles.

Inductively coupled plasma-optical emission spectrometry (ICP-OES) is a quantitative and powerful tool for metal determination in a variety of matrices (Hou and Jones 2012). In Figure $6.6 \mathrm{~b}$ the results for copper elemental quantification using ICP-OES on CMC- and TNFC-copper nanoparticle materials are presented. As observed in Figure 6.6b, copper content in all dried CMC-copper nanoparticles materials was reduced compared with the original aqueous hybrid CMC-copper nanoparticles. Copper content decreased up to 68.5\% in the spray-dried material; $23.5 \%$ in the freeze dried material and $49.4 \%$ for the solvent exchange with ethanol and butanol pre-freeze drying process (E/1-B-FD). Copper content differences among all CMC-copper nanoparticles for both aqueous and dried materials was significantly different $\quad\left(p\right.$-value $\left.=9.83^{\times} 10^{-6}<0.05\right)$.

The spray-drying process caused more copper loss than the freeze-drying process. This phenomenon might happen because of the additional steps required to perform a spray drying process compared to the freeze drying process. Spray drying consists on the following four steps: 1) atomization of liquid into droplet, 2) contact of the droplets with 
the hot drying gas, 3) droplets drying into particles in the drying chamber, and 4) separation of the particles from the drying gas in the cyclone chamber (Gil et al. 2010; Peng et al. 2012b). The final drying gas passes through the chamber and vent to appropriated waste stream. We assume that especially during stages 3 and 4 of spray drying, the continuous air flow and the strong cyclone forces applied on particles were responsible for copper leaching. More investigation must be performed in this regard to clarify these copper losses.

The copper content of samples exposed to solvent exchange with ethanol/butanol previous freeze drying also exhibited a reduction in copper, but not as high as those spray-dried samples. Modified freeze drying consists of three stages; copper loss might be attributed to the solvent exchange pretreatment, specifically during the process of centrifugation (Xia et al. 2006).

Copper content in CMC substrate presents the following trend: freeze-drying > modified freeze-drying > spray-drying.

Copper content in TNFC substrates presents a similar behavior compared to CMC substrates; that is, copper content decreases after the drying processes (Figure 6.6b), but the reduction is less dramatic than for CMC substrates. In the hybrid TNFC-copper nanoparticles, copper content decreased up to $10.1 \%$ for the freeze material and up to $14.5 \%$ for those solvent exchange samples previous freeze trying (E/tert-B-FD). This reduction is not statistically significant; that is, copper content among all TNFC-copper nanoparticles, that is aqueous and dried materials, are not significantly different ( $p$-value $=0.053^{>0} 0.05$ ).

\subsubsection{Oxidation state of copper CMC-copper nanoparticles and TNFC-copper nanoparticles using X-ray photoelectron spectroscopy (XPS)}


During the production of the hybrid materials CMC- and TNFC-copper nanoparticles, the suspensions experimented changes in color indicating changes in the copper's state of oxidation. In general, in aqueous suspensions, those changes were from light/dark blue (presence of $\mathrm{Cu}^{2+}$ ) to dark brown (presence of metallic copper and or $\mathrm{Cu}^{1+}$ ). Similar phenomenon was reported by Cady et al. (2011). After the spray- and freeze-drying process, the CMC-copper nanoparticles exhibited a yellow and green color, respectively. On the contrary, all dried TNFC-copper nanoparticles resulted in a light blue color.

XPS was used to determine the oxidation state of copper materials after the drying process. In measuring the core electrons of copper element there are two characteristic peaks classified as the $\mathrm{Cu} 2 \mathrm{p}_{1 / 2}$ and $\mathrm{Cu} 2 \mathrm{p}_{3 / 2}$ at energy levels near to $952 \mathrm{eV}$ and $932 \mathrm{eV}$, respectively. In Figures 6.7-6.8 the XPS spectra of CMC and TNFC samples containing copper nanoparticles after the drying process are presented; the two characteristic peaks $\mathrm{Cu} 2 \mathrm{p}_{1 / 2}$ and $\mathrm{Cu} 2 \mathrm{p}_{3 / 2}$ can be clearly observed in the expanded spectrum (Figures' inserts) which confirm the presence of copper in the surface of all the cellulosic materials.

To determine the actual oxidation state of copper in the cellulosic materials, a comparison of the characteristic binding energy for $\mathrm{Cu}_{2} \mathrm{p}_{3 / 2}$, presented in Table 6.2, and the resulting binding energies, presented in Table 6.3, was measured. Based on this analysis we can conclude that copper present on the SD-, FD- and E/1-B-FD CMC materials are $\mathrm{Cu}^{0}$ or $\mathrm{Cu}^{+}$ (binding energies closed to $932.5 \mathrm{eV}$ ). Unfortunately, $\mathrm{Cu}^{0}$ or $\mathrm{Cu}^{+}$cannot be distinguished from one another based on XPS analysis only since both peaks appear in the range between $932 \mathrm{eV}$ and $933 \mathrm{eV}$ and the $\mathrm{Cu} 2 \mathrm{p}_{3 / 2}$ bands for the two species are separated by $0.1 \mathrm{eV}$ (Cady et al. 2011). The absence of $\mathrm{Cu} 2 \mathrm{p}_{3 / 2}$ peaks between $933 \mathrm{eV}$ and $934 \mathrm{eV}$ suggests that no $\mathrm{Cu}^{2+}$ is present in the dried CMC-copper nanoparticles samples.

In terms of the oxidation state of copper for the TNFC dried materials, the characteristic binding energies for $\mathrm{Cu} 2 \mathrm{p}_{3 / 2}$ of both FD- and E/tert-B-FD TNFC-copper nanoparticles appear between the bands between $933 \mathrm{eV}$ and $934 \mathrm{eV}$ (Table 6.3) suggesting that $\mathrm{Cu}^{2+}$ is present in the surfaces of the dried TNFC-copper nanoparticles materials. 

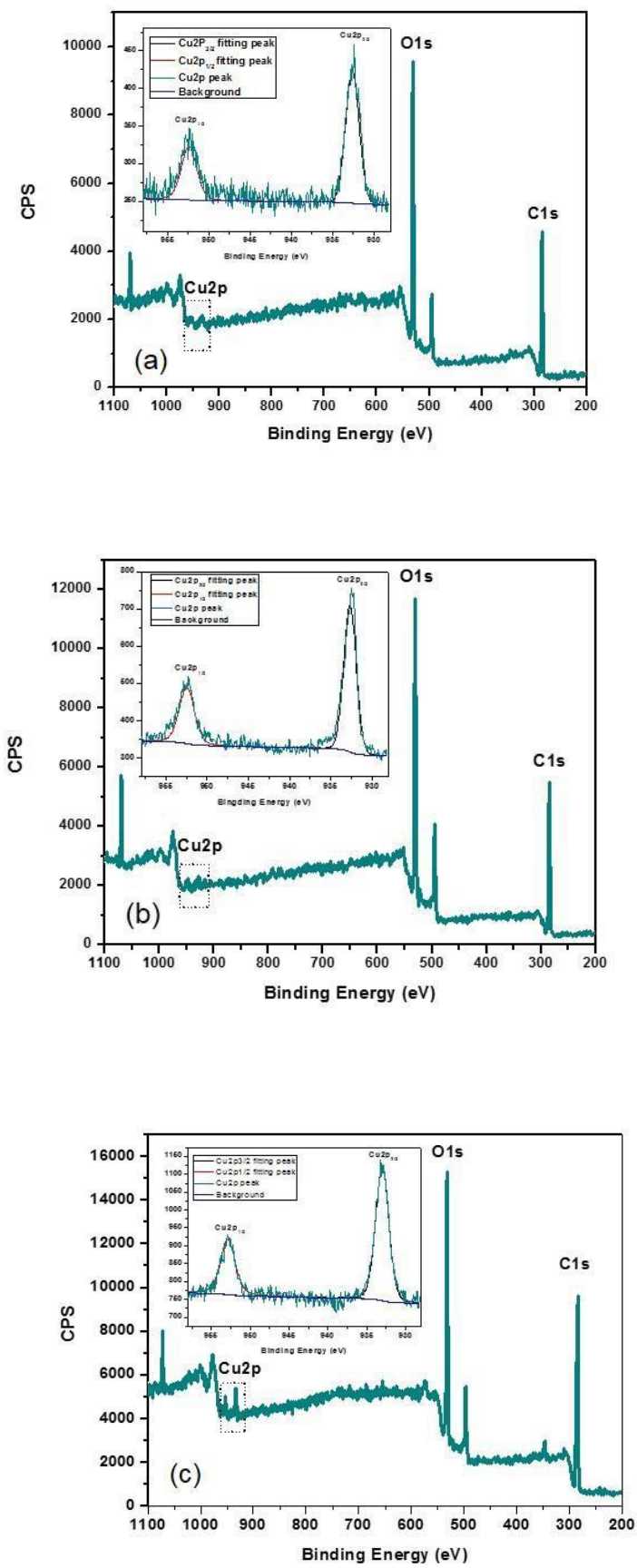

Figure 6. 7 XPS full survey scan of (a) SD CMC-copper nanoparticles, (b) FD CMCcopper nanoparticles and (c) E/1-B-FD CMC-copper nanoparticles. Inset: $\mathrm{Cu} 2 \mathrm{p}_{3 / 2}$ and $\mathrm{Cu} 2 \mathrm{p}_{1 / 2}$ XPS detail scan. 

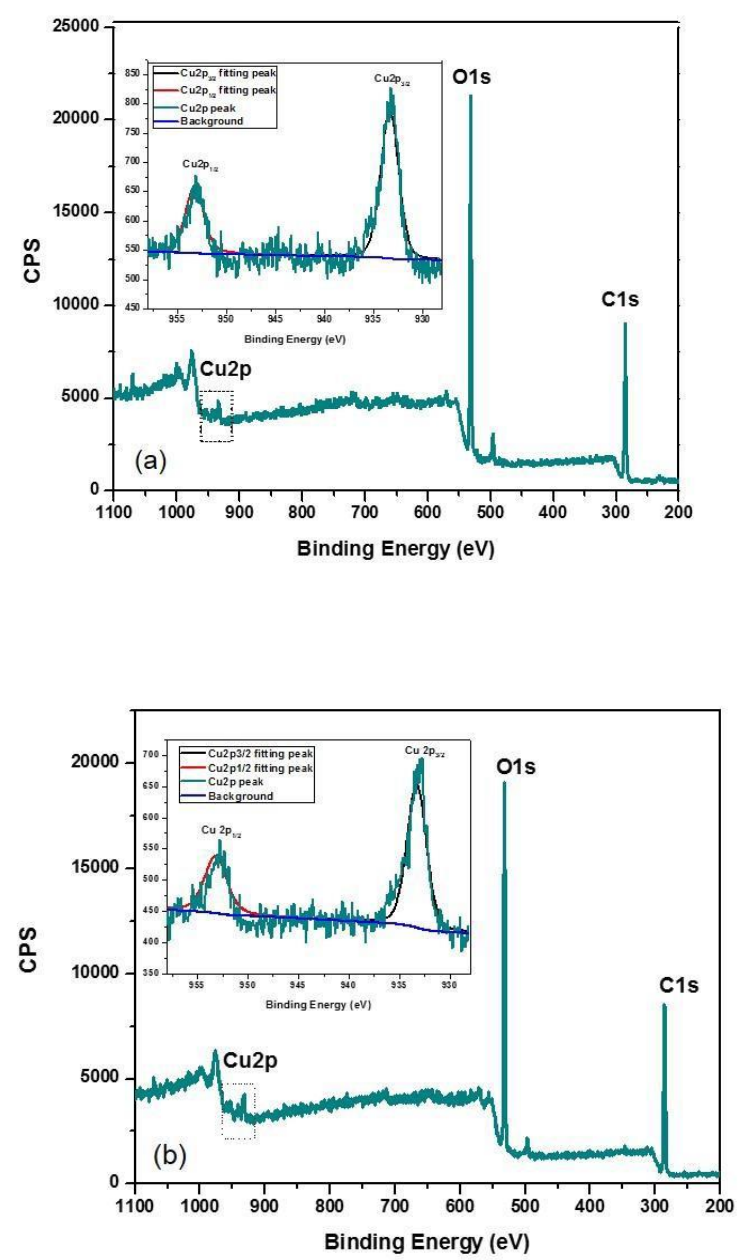

Figure 6. 8 XPS full survey scan of (a) FD TNFC-copper nanoparticles, and (c) E/tert-BFD TNFC-copper nanoparticles. Inset: $\mathrm{Cu} 2 \mathrm{p}_{3 / 2}$ and $\mathrm{Cu} 2 \mathrm{p}_{1 / 2}$ XPS detail scan.

Table 6. 2 Binding energy $\left(\mathrm{Cu} 2 \mathrm{p}_{3 / 2}\right)$ for copper different states of oxidation (Mclntyre and Cook 1975).

\begin{tabular}{ccc}
\hline Chemical state & Formula & $\begin{array}{c}\text { Binding energy, } \mathrm{Cu} 2 \mathrm{p}_{3 / 2} \\
(\mathrm{eV})\end{array}$ \\
\hline $\mathrm{Cu}$ metal or $\left(\mathrm{Cu}^{0}\right)$ & $\mathrm{Cu}$ & $932.5 \pm 0.15$ \\
$\mathrm{Cu}(\mathrm{I})$ oxide or $\left(\mathrm{Cu}^{+1}\right)$ & $\mathrm{Cu}_{2} \mathrm{O}$ & $932.5 \pm 0.2$ \\
$\mathrm{Cu}(\mathrm{II})$ oxide or $\left(\mathrm{Cu}^{+2}\right)$ & $\mathrm{CuO}$ & $933.8 \pm 0.2$ \\
$\mathrm{Cu}$ hydroxide or $\left(\mathrm{Cu}^{+2}\right)$ & $\mathrm{Cu}(\mathrm{OH})_{2}$ & $934.4 \pm 0.2$ \\
\hline
\end{tabular}


Table 6. 3 Summary of binding energy for $\mathrm{Cu} 2 \mathrm{p}_{3 / 2}$.

\begin{tabular}{cc}
\hline Samples & Binding Energy $(\mathrm{eV})$ for $\mathrm{Cu} 2 \mathrm{p}_{3 / 2}$ \\
\hline SD CMC-copper nanoparticles & 932.77 \\
FD CMC-copper nanoparticles & 932.71 \\
E/1-B-FD CMC-copper nanoparticles & 932.98 \\
FD TNFC-copper nanoparticles & 933.33 \\
E/tert-B-FD TNFC-copper nanoparticles & 933.27 \\
\hline \hline
\end{tabular}

\subsubsection{Crystal structures of CMC-copper nanoparticles and TNFC-copper nanoparticles using X-ray diffraction (XRD)}

The results from XRD are consistent with the XPS analysis and confirms the existence of $\mathrm{Cu}_{2} \mathrm{O}\left(\mathrm{Cu}^{+}\right)$crystallites in all CMC-copper samples (SD, FD and E/1-B-FD). In Figure $6.9 \mathrm{a}, \mathrm{b}$ the XRD patterns of CMC-copper samples matching the most prominent characteristic peaks of $\mathrm{Cu}_{2} \mathrm{O}$ at $2 \theta=36.5^{\circ}$ are presented (International Center for Diffraction Data (ICDD): 01-077-0199) (See Table 6.4). The impurities sodium sulfate crystallites (ICDD: 00-024-1132) were also found in all CMC-copper samples as shown in Figure 6.9a, b (inside the square), which might be introduced from the byproducts in the reaction between $\mathrm{CMC}$ and cupric sulfate in this study.

The results indicate that $\mathrm{SD}$ may favor the formation of $\mathrm{Cu}_{2} \mathrm{O}$ crystallites since the SD sample has the greatest intensity and numbers of characteristic peaks of $\mathrm{Cu}_{2} \mathrm{O}$, compared to the other two CMC-copper samples. The E/1-B-FD CMC-copper sample has the least intensity and numbers of characteristic peaks of $\mathrm{Cu}_{2} \mathrm{O}$ possibly because of higher solubility of $\mathrm{Cu}_{2} \mathrm{O}$ in the alcohol solvents. Therefore, in terms of forming $\mathrm{Cu}_{2} \mathrm{O}$ crystallites in $\mathrm{CMC}$ copper materials the effectiveness of the three drying methods used in this study from the greatest to the least is: $\mathrm{SD}>\mathrm{FD}>\mathrm{E} / 1-\mathrm{B}-\mathrm{FD}$.

Unusual crystal morphologies that possess numerous edge/corner and other reactive surface sites increased antimicrobial properties (Stoimenov et al. 2002). We speculate that having more $\mathrm{Cu}_{2} \mathrm{O}$ crystals on SD- and FD CMC might contribute positively in their antimicrobial performance. 
The XRD pattern of freeze-dried TNFC shows that the intense peaks at $2^{\theta}=16.2^{\circ}$, $22.7^{\circ}$, and $34.0^{\circ}$ as shown in Figure $6.9 \mathrm{c}$ are the characteristic peaks for the crystalline planes of (-110), (200), (004) of cellulose, which is consistent with reported literature value (Zhao et

al. 2007; Peng 2013c). An unidentified peak with high intensity was observed at $2 \theta$ around $44^{\circ}$. It is important to note that the material in analysis is not pure cellulose; the material was previously chemically modified to get the final TNFC, so impurities are expected to be

found. We speculate that the peak at $2 \theta$ around $44^{\circ}$ might be assigned to sodium ions in the freeze-dried TNFC. This issue will require further investigation. No copper typical peaks are present which indicates that freeze-dried process was not favorable to the formation of copper nanoparticles when nanofibrillated cellulose was used as the substrate.

Table 6. 4 Typical XRD parameters for copper and cellulose according to its chemical state (International Center for Diffraction Data (ICDD).

\begin{tabular}{|c|c|c|c|c|}
\hline Chemical state & Formula & 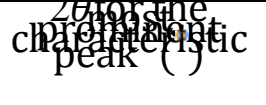 & $\begin{array}{l}\text { Corresponding } \\
\text { crystalline plane }\end{array}$ & $\begin{array}{c}\text { ICDD } \\
\text { Card No. }\end{array}$ \\
\hline $\mathrm{Cu}$ metal or $\left(\mathrm{Cu}^{0}\right)$ & $\mathrm{Cu}$ & 43.3 & (111) & $\begin{array}{c}\text { 01-085- } \\
1326\end{array}$ \\
\hline $\begin{array}{c}\mathrm{Cu}(\mathrm{I}) \text { oxide or } \\
\left(\mathrm{Cu}^{+}\right)\end{array}$ & $\mathrm{Cu}_{2} \mathrm{O}$ & 36.5 & (111) & $\begin{array}{c}01-077- \\
0199\end{array}$ \\
\hline $\begin{array}{l}\mathrm{Cu} \text { (II) oxide or } \\
\left(\mathrm{Cu}^{2+}\right)\end{array}$ & $\mathrm{CuO}$ & 35.6 & $(002)$ & $\begin{array}{c}01-089- \\
5899\end{array}$ \\
\hline Cellulose & $\left(\mathrm{C}_{6} \mathrm{H}_{10} \mathrm{O}_{5}\right)_{\mathrm{n}}$ & 22 & $(200)$ & ------ \\
\hline
\end{tabular}



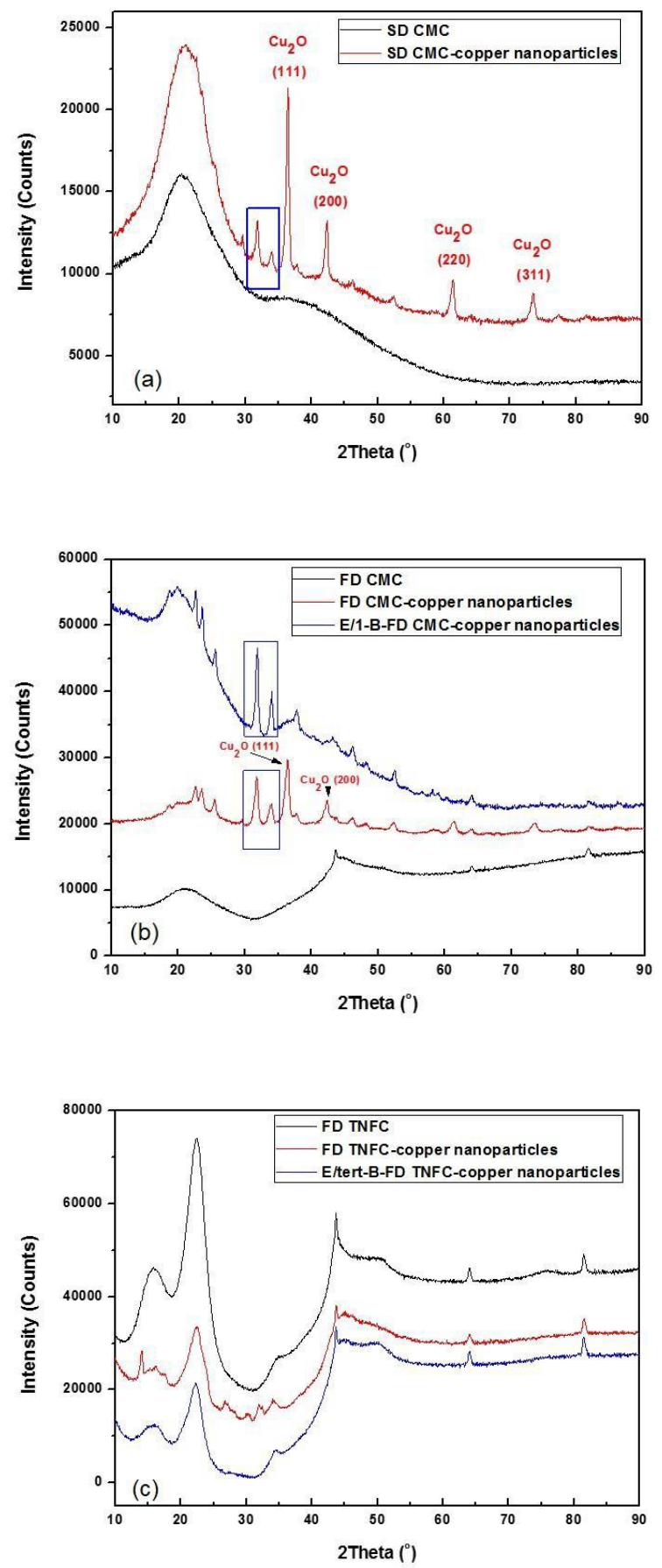

Figure 6. 9 XRD patterns of (a) CMC based materials obtained from SD process, (b) CMC based materials obtained FD and E/1-B-FD processes and (c) TNFC based materials obtained from FD and E/tert-B-FD processes. 
Four additional peaks at $2^{\theta}=14.1^{\circ}, 26.7^{\circ}, 30.5^{\circ}$ and $31.9^{\circ}$ in the XRD pattern of FD TNFC- copper nanoparticles (Figure 6.9c) are difficult to assign crystalline behavior, all these peaks are not assigned to crystalline planes of $\mathrm{Cu}^{0}, \mathrm{Cu}_{2} \mathrm{O}$ nor $\mathrm{CuO}$. The XPS result shows the presence of $\mathrm{Cu}^{2+}$ in the FD TNFC-copper nanoparticles material, so we can speculate that these peaks might be attributed to crystalline planes of $\mathrm{Cu}^{2+}$ TNFC complex. These peaks at $2^{\theta}=14.1^{\circ}, 26.7^{\circ}, 30.5^{\circ}$ and $31.9^{\circ}$ disappeared in the XRD pattern of E/tert-B-FD TNFC-copper nanoparticles materials, suggesting no crystalline planes of copper- containing compound in the E/tert-B-FD TNFC-copper nanoparticles material. The disappearance of peaks for the crystalline planes of postulated $\mathrm{Cu}^{2+} \mathrm{TNFC}$ complex might be because of additional solvent exchange process, which caused copper loss or the dissolution of crystals in the organic solvents. $\mathrm{Cu}^{2+}$ complex also demonstrated good antimicrobial properties (Beeton et al. 2014), however, copper nanoparticles have been demonstrated to possess higher antimicrobial activities than the equivalent amount of copper containing compounds such as $\mathrm{CuCl}_{2}$ because copper nanoparticles have larger surface to volume ratio, crystalline structure. The more reactive sites of copper nanoparticles therefore could produce more reactive oxygen species (ROS) that could result in considerable lipid peroxidation, protein oxidation and DNA degradation and finally killing the cells (Rai et al. 2009; Chatterjee et al. 2014). In term of favoring the formation of copper crystallites in the cellulose substrates, the most effectiveness of substrates used in this study is in the order: CMC > TNFC.

\subsubsection{Estimation of crystallite size using XRD data}

Full width at half maximum (FWHM) of the XRD peaks is related to the characteristic crystallite size (Zanchet et al. 2000). Therefore FWHM was used to estimate the crystallite size of copper nanoparticles in spray-, freeze- and modified freeze dried materials. To better evaluate crystallite size, the calculation often can obtain better results from the most intense peak for the crystalline plane of copper particles. The most intense XRD peak for the crystalline plane of cubic $\mathrm{Cu}_{2} \mathrm{O}$ in either SD- or FD CMC-copper nanoparticles was at $2 \theta=36.5^{\circ}$ corresponding to the (111) plane. The FWHM values from the peaks for the 
crystalline plane (111) were used to calculate the crystallite size. The estimated average crystallite size of $\mathrm{Cu}_{2} \mathrm{O}$ on SD CMC-copper nanoparticles material was $29.6 \pm 0.002 \mathrm{~nm}$ for the (111) crystalline plane. The estimated average crystallite size of $\mathrm{Cu}_{2} \mathrm{O}$ on FD CMC- copper nanoparticles is $14.2 \pm 5.1 \mathrm{~nm}$. We assume that the high standard deviation in the calculation of the crystallite size of copper nanoparticles on FD sample might be attributed to the sample heterogeneity, and the shape and size distribution of the crystallites. The intensity of the peak for (111) crystalline planes of $\mathrm{Cu}_{2} \mathrm{O}$ was too weak to be used for estimating the crystallite size on E/1-B-FD CMC-copper nanoparticles. Smaller crystals and having more crystal structures exhibit higher antimicrobial activities than big ones and having less crystals because of their larger surface to volume ratio that allows them to interact closely with bacterial membranes (Stoimenov et al. 2002; Azam et al. 2012). Smaller copper crystallites and more crystallites on cellulose substrates would probably contribute to higher antimicrobial activity of hybrid cellulose-copper materials.

For all dried TNFC-copper nanparticles materials, there were no obvious peaks for crystalline planes of copper particles (neither $\mathrm{Cu}, \mathrm{Cu}_{2} \mathrm{O}$ nor $\mathrm{CuO}$ ) in the XRD patterns, therefore the crystallite size data was not obtained for all dried TNFC-copper nanoparticles.

\subsection{Conclusions}

The final morphologies were different among spray-dried (SD), freeze-dried (FD) and modified freeze-dried cellulose-copper nanoparticle materials. The SD material exhibited spherical shapes and FD material showed plate-like structures. The particle size of SD materials were significantly reduced compared with the FD materials. The particle size of the SD materials ranged from several hundred nanometers to microns. The length and width of plate-like FD materials were over hundreds of microns.

Copper generates a porous structure on both CMC and TNFC substrates; this porous characteristic is enhanced after the solvent exchange pre-treatment. Bundle-like and 
pororus structures were observed for the carboxymethyl cellulose (CMC) substrate when 
using ethanol and "1-butanol" solvent exchange process followed by the freeze drying step. A film-like and porous structure was exhibited for TEMPO nanofibrillated cellulose when using ethanol and "tert-butanol" solvent exchange process followed by freeze drying.

The inductively coupled plasma-optical emission spectrometry (ICP-OES) data shows that the spray-drying process caused most copper losses compare to the freeze and modified freeze-dried processes. The copper content on CMC-copper nanoparticles material decreased by $68.5 \%$ when using the spray-drying process. It is assumed that the additional steps performed during the spray-drying process is the main cause of the extra loss in copper.

The XPS analysis confirms that $\mathrm{Cu}^{+}$or $\mathrm{Cu}^{0}$ is present for all CMC-copper nanoparticle materials; $\mathrm{Cu}^{2+}$ was not detected in these samples. On the contrary, $\mathrm{Cu}^{2+}$ was found for all TNFC-copper nanoparticle materials. The XRD results shows that the copper nanoparticles in the $\mathrm{CMC}$ substrate is $\mathrm{Cu}_{2} \mathrm{O}$, and the most effectiveness of drying methods to form copper crystallites on CMC is the spray-drying process. The desirable substrate for favoring the formation of copper crystallites on the cellulose substrate is CMC compared to the TNFC material.

The XRD data indicate that the average crystallite size of $\mathrm{Cu}_{2} \mathrm{O}$ on SD CMC-copper nanoparticles material is $29.6 \mathrm{~nm}$, while on FD CMC-copper nanoparticles material the crystallite size is $14.2 \mathrm{~nm}$. The intensity of the peaks corresponding to crystalline planes of $\mathrm{Cu}_{2} \mathrm{O}$ was too weak to be used to estimate the crystallite size for E/1-B-FD CMC-copper nanoparticles. No evident crystal structure of copper nanoparticles or copper containing compounds were found in those dried TNFC-copper nanoparticles; therefore, crystallite size data were not obtained. 


\subsection{References}

Abdelwahed, W., Degobert, G., Stainmesse, S., \& Fessi, H. (2006). Freeze-drying of nanoparticles: formulation, process and storage considerations. Advanced Drug Delivery Reviews, 58(15), 1688-1713.

Azam, A., Ahmed, A. S., Oves, M., Khan, M. S., \& Memic, A. (2012). Size-dependent antimicrobial properties of $\mathrm{CuO}$ nanoparticles against Gram-positive and-negative bacterial strains. International Journal of Nanomedicine, 7, 3527.

Beeton, M. L., Aldrich-Wright, J. R., \& Bolhuis, A. (2014). The antimicrobial and antibiofilm activities of copper (II) complexes. Journal of Inorganic Biochemistry, 140, 167-172.

Borchert, H., Shevchenko, E. V., Robert, A., Mekis, I., Kornowski, A., Grübel, G., \& Weller, H. (2005). Determination of nanocrystal sizes: a comparison of TEM, SAXS, and XRD studies of highly monodisperse CoPt3 particles. Langmuir, 21(5), 1931-1936.

Cady, N. C., Behnke, J. L., \& Strickland, A. D. (2011). Copper-based nanostructured coatings on natural cellulose: nanocomposites exhibiting rapid and efficient inhibition

of a multi-drug resistant wound pathogen, A. baumannii, and mammalian cell biocompatibility in vitro. Advanced Functional Materials, 21(13), 2506-2514.

Chatterjee, A. K., Chakraborty, R., \& Basu, T. (2014). Mechanism of antibacterial activity of copper nanoparticles. Nanotechnology, 25(13), 135101.

Djurado, E., Bouvier, P., \& Lucazeau, G. (2000). Crystallite size effect on the tetragonalmonoclinic transition of undoped nanocrystalline zirconia studied by XRD and Raman spectrometry. Journal of Solid State Chemistry, 149(2), 399-407. 
Eichhorn, S. J., Dufresne, A., Aranguren, M., Marcovich, N. E., Capadona, J. R., Rowan, S. J., ... \& Peijs, T. (2010). Review: current international research into cellulose nanofibres and nanocomposites. Journal of Materials Science, 45(1), 1-33.

Elanthikkal, S., Gopalakrishnapanicker, U., Varghese, S., \& Guthrie, J. T. (2010). Cellulose microfibres produced from banana plant wastes: Isolation and characterization. Carbohydrate Polymers, 80(3), 852-859.

Ferreira, T., Rasband, W. (2012). ImageJ user guide IJ 1.46r. Research Services Branch Web. http://imagej.nih.gov/ij/docs/guide/user-guide.pdf. Accessed 02 October 2012

Gardner, D. J., Oporto, G. S., Mills, R., \& Samir, M. A. S. A. (2008). Adhesion and surface issues in cellulose and nanocellulose. Journal of Adhesion Science and Technology, 22(5-6), 545-567.

Gil, M., Vicente, J., \& Gaspar, F. (2010). Scale-up methodology for pharmaceutical spray drying. Chimica Oggi/Chemistry Today, 28(4).

Hou, X., \& Jones, B. T. (2000). Inductively Coupled Plasma-Optical Emission Spectrometry. Encyclopedia of Analytical Chemistry.

Hult, E. L., Larsson, P. T., \& Iversen, T. (2001). Cellulose fibril aggregation —an inherent property of kraft pulps. Polymer, 42(8), 3309-3314.

Jin, H., Nishiyama, Y., Wada, M., \& Kuga, S. (2004). Nanofibrillar cellulose aerogels. Colloids and surfaces A: Physicochemical and engineering aspects, 240(1), 63-67. 
Klemm, D., Kramer, F., Moritz, S., Lindström, T., Ankerfors, M., Gray, D., \& Dorris, A. (2011). Nanocelluloses: a new family of nature-based materials. Angewandte Chemie International Edition, 50(24), 5438-5466.

Liu, M. S., Lin, M. C., Huang, I. T., \& Wang, C. C. (2006). Enhancement of thermal conductivity with $\mathrm{CuO}$ for nanofluids. Chemical Engineering \& Technology, 29(1), 7277.

Markowicz, A. (2011). An overview of quantification methods in energy-dispersive X-ray fluorescence analysis. Pramana, 76(2), 321-329.

McIntyre, N. S., \& Cook, M. G. (1975). X-ray photoelectron studies on some oxides and hydroxides of cobalt, nickel, and copper. Analytical Chemistry, 47(13), 2208-2213.

Nandiyanto, A. B. D., \& Okuyama, K. (2011). Progress in developing spray-drying methods for the production of controlled morphology particles: from the nanometer to submicrometer size ranges. Advanced Powder Technology, 22(1), 1-19

Pakowski, Z. (2007). Modern methods of drying nanomaterials. Transport in Porous Media, 66(1-2), 19-27.

Peng, Y., Gardner, D. J., \& Han, Y. (2012a). Drying cellulose nanofibrils: in search of a suitable method. Cellulose, 19(1), 91-102.

Peng, Y., Han, Y., \& Gardner, D. J. (2012b). Spray-drying cellulose nanofibrils: effect of drying process parameters on particle morphology and size distribution. Wood and Fiber Science, 44(4), 448-461. 
Peng, Y., Gardner, D. J., Han, Y., Cai, Z., \& Tshabalala, M. A. (2013a). Influence of drying method on the surface energy of cellulose nanofibrils determined by inverse gas chromatography. Journal of Colloid and Interface Science, 405, 85-95.

Peng, Y., Gardner, D. J., Han, Y., Kiziltas, A., Cai, Z., \& Tshabalala, M. A. (2013b). Influence of drying method on the material properties of nanocellulose I: thermostability and crystallinity. Cellulose, 20(5), 2379-2392.

Peng, Y. (2013c). Cellulose nanofibrils drying and their utilization in reinforcing thermoplastic composites. The University of Maine.

Rai, M., Yadav, A., \& Gade, A. (2009). Silver nanoparticles as a new generation of antimicrobials. Biotechnology Advances, 27(1), 76-83.

Sehaqui, H., Zhou, Q., \& Berglund, L. A. (2011). High-porosity aerogels of high specific surface area prepared from nanofibrillated cellulose (NFC). Composites Science and Technology, 71(13), 1593-1599.

Sehaqui, H., Zhou, Q., Ikkala, O., \& Berglund, L. A. (2011). Strong and tough cellulose nanopaper with high specific surface area and porosity. Biomacromolecules, 12(10), 3638-3644.

Stoimenov, P. K., Klinger, R. L., Marchin, G. L., \& Klabunde, K. J. (2002). Metal oxide nanoparticles as bactericidal agents. Langmuir, 18(17), 6679-6686.

Xia, X., Xie, C., Cai, S., Yang, Z., \& Yang, X. (2006). Corrosion characteristics of copper microparticles and copper nanoparticles in distilled water. Corrosion Science, 48(12), 3924-3932. 
Zanchet, D., Hall, B. D., \& Ugarte, D. (2000). Structure population in thiol-passivated gold nanoparticles. The Journal of Physical Chemistry B, 104(47), 11013-11018.

Zhao, H., Kwak, J. H., Zhang, Z. C., Brown, H. M., Arey, B. W., \& Holladay, J. E. (2007). Studying cellulose fiber structure by SEM, XRD, NMR and acid hydrolysis. Carbohydrate Polymers, 68(2), 235-241.

Zhong, T., Oporto, G. S., Jaczynski, J., Tesfai, A. T., \& Armstrong, J. (2013). Antimicrobial properties of the hybrid copper nanoparticles-carboxymethyl cellulose. Wood and Fiber Science, 45(2), 215-222. 


\section{CHAPTER 7: CONCLUSIONS AND REMARKS ON FUTURE RESEARCH}

\subsection{Conclusions}

In this work, two cellulosic materials [carboxymethyl cellulose (CMC) and TEMPO nanofibrillated cellulose (TNFC)] have been used as templates for the synthesis of copper nanoparticles and the resulting hybrid material demonstrated effective to be used as antimicrobial agent for active packaging applications.

The following are the summary of conclusions of this research work:

(1) CMC can be effectively used as a starting material to synthesize in situ copper nanoparticles via chemical reduction of copper ions on the cellulose surface using sodium borohydride as a reducing agent. The copper nanoparticles with the diameter of 10- and $20 \mathrm{~nm}$ were distributed on the cellulose surface. The antimicrobial activity indicated that 10-h exposure of the 18-h E. coli culture to CMC-copper nanoparticles suspension resulted in at least an 8-log microbial reduction, indicating that the final hybrid material possesses effective antimicrobial properties against E. coli DH5 $\alpha$, a nonpathogenic surrogate foodborne pathogen.

(2) The hybrid materials suspension can directly mix with polar and hydrophilic thermoplastic resin. The hydrophilic polyvinyl alcohol composite films incorporating the hybrid TNFC-copper nanoparticles were successfully prepared by solvent casting of the mixture of PVA solution and TNFC-copper nanoparticles suspension. The influences of the contents of copper nanoparticles on the antimicrobial and thermal properties have been investigated. Increasing the content of copper nanoparticles into the PVA/TNFC matrix resulted into greater bacterial reduction, when the copper loading in the matrix reached 0.6 wt.\%, the bacterial reduction dramatically increased, resulting in up to 5-log microbial reduction. The PVA composite films with TNFC-copper nanoparticles exhibited improved thermal stability, the decomposition temperature, corresponding to the 
maximum weight loss, increased from $260{ }^{\circ} \mathrm{C}$ for pure PVA, to $278{ }^{\circ} \mathrm{C}$ for the PVA composite with 0.6 wt.\% copper nanoparticles.

(3) PVA composite film containing carboxymethyl cellulose and 0.6 wt.\% copper nanoparticles was successfully prepared in wet process. The antimicrobial and copper ion release properties as function of exposure time for both PVA/CMC-Cu0.6 and PVA/TNFC-Cu0.6 have been investigated. The number of survival E.coli on both PVA composite films decreased with the increasing exposure time. Both PVA composite films

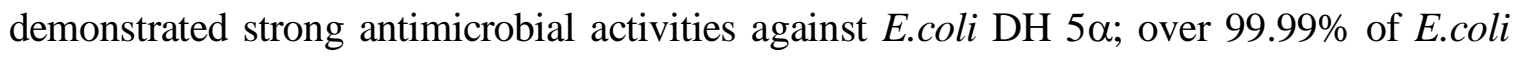
were inactivated after 3-day exposure of E.coli to either PVA/TNFC-Cu0.6 film or PVA/CMC-Cu0.6 film. Approximately 90\% less cellulosic material was required when using TNFC as copper template compared to CMC template to get similar films antimicrobial performance. Measurements of antimicrobial and copper ion release revealed that there is no direct evidence showing correlation between antimicrobial properties and the amount of copper ion release. PVA embedded with CMC-copper nanoparticles inactivated more E.coli $\mathrm{DH} 5 \alpha$ at the same exposure time compared with PVA containing TNFC-copper nanoparticles. That might be attributed to higher amount of crystalline $\mathrm{Cu}_{2} \mathrm{O}$ in PVA/CMC matrix. The incorporation of TNFC-copper nanoparticles and CMC-copper nanoparticles on the PVA matrix increased the maximum decomposition temperature by 18 ${ }^{\circ} \mathrm{C}$ and $25^{\circ} \mathrm{C}$ compared to pure PVA, respectively. The storage modulus of the PVA composite films at $48{ }^{\circ} \mathrm{C}$ increased up to $164 \%$ and $217 \%$ with the incorporation of TNFCcopper nanoparticles and CMC-copper nanoparticles, respectively.

(4) Freeze-dried (FD) hybrid materials attempted to be incorporated into nonpolar thermoplastic polypropylene (PP) and polylactic acid (PLA) matrices using a twin-screw extrusion followed by both injection molding process and a film formation process (dry process). The incorporation of FD hybrid materials was found to compromise the tensile strength of PP and PLA composites, leading to the decrease up to $19.2 \%$ for PP composite and $12.1 \%$ for PLA composite. SEM analysis showed that the presence of fiber agglomerates in the matrices and therefore the poor interfacial adhesion between them and 
matrices might be the most important reason for this mechanical property reduction. The agglomerations might be produced mainly during the freeze drying process. The crystallinity of PP and PLA were found to increase after the incorporation of FD hybrid materials, which is consistent with the literature that cellulose based materials have favored nucleation and crystal growth. No copper ion release was found from extruded-injected PP and PLA composites after being immersed in an aqueous medium for a month. Low amount of copper ions were found to leach from PLA thin film that was prepared by extrusion followed by film formation processes. No antimicrobial activities of PLA composite films were found, that might be as a consequence of insufficiency of copper ions leaching from the film.

(5) Removing water from the hybrid cellulose-copper nanoparticles suspension is a critical step before incorporating the hybrid materials into hydrophobic thermoplastic resins since water in the hybrid materials is a detriment to satisfactory processing during hot melting extrusion. Three drying methods (spray drying, freeze drying and modified freeze drying) for hybrid materials suspensions have been investigated. a), the morphologies of the dried hybrid materials were found to be dependent on the drying methods. The spray-dried (SD) material exhibited spherical shapes and freeze dried (FD) material showed plate-like structures. The particle size of SD materials were significantly reduced compared with the FD materials. The particle size of the SD materials ranged from several hundred nanometers to microns. The length and width of plate-like FD materials were over hundreds of microns. Copper generates a porous structure on both CMC and TNFC substrates; this porous characteristic is enhanced after the solvent exchange pre-treatment. Bundle-like and porous structures were observed for the carboxymethyl cellulose (CMC) substrate when using ethanol and "1-butanol" solvent exchange process followed by the freeze drying step. A film-like and porous structure was exhibited for TEMPO nanofibrillated cellulose when using ethanol and "tert-butanol" solvent exchange process followed by freeze drying. b), the final copper contents on cellulose substrates were also found to be significantly affected by the drying methods. Spray drying process caused the most copper loss (up to $68.5 \%$ loss), the freeze drying caused least copper loss. c), 
cellulose substrates were also found to have significantly influences on crystalline structures and oxidation states of copper on the cellulose. The XPS analysis confirms that $\mathrm{Cu}^{+}$or $\mathrm{Cu}^{0}$ is present for all CMC-copper nanoparticle materials; $\mathrm{Cu}^{2+}$ was not detected in these samples. On the contrary, $\mathrm{Cu}^{2+}$ was found for all TNFC-copper nanoparticle materials. The XRD results shows that the copper nanoparticles in the CMC substrate is $\mathrm{Cu}_{2} \mathrm{O}$, and the most effectiveness of drying methods to form copper crystallites on CMC is the spray-drying process. The desirable substrate for favoring the formation of copper crystallites on the cellulose substrate is CMC compared to the TNFC material. The XRD data indicates that the average crystallite size of $\mathrm{Cu}_{2} \mathrm{O}$ on SD CMC-copper nanoparticles material is $29.6 \mathrm{~nm}$, while on FD CMC-copper nanoparticles material the crystallite size is $14.2 \mathrm{~nm}$. The intensity of the peaks corresponding to crystalline planes of $\mathrm{Cu}_{2} \mathrm{O}$ was too weak to be used to estimate the crystallite size for E/1-B-FD CMC-copper nanoparticles. No evident crystal structure of copper nanoparticles or copper containing compounds were found in those dried TNFC-copper nanoparticles; therefore, crystallite size data were not obtained.

\subsection{Remarks on future research}

Although an 8-log microbial reduction in Chapter 2, a 5-log microbial reduction in chapter 4 and a 6-log microbial reduction in chapter 5 are encouraging, it needs to be emphasized that $E$. coli $\mathrm{DH} 5 \alpha$ is nonpathogenic with a high sensitivity to various stresses, unlike E. coli $\mathrm{O} 157: \mathrm{H} 7$, which is a typical foodborne pathogen with a higher resistance. E. coli DH5 $\alpha$ was used in this study as a nonpathogenic surrogate for the pathogenic E. coli $\mathrm{O} 157: \mathrm{H} 7$. The use of nonpathogenic bacterial surrogates is standard procedure in the initial determination of novel antimicrobial treatments such as in this study. I recommend that in the future study foodborne pathogens such as Gram-negative E. coli O157:H7 and Gram-positive Staphylococcus aureus could be used as target bacteria for antimicrobial testing of the produced polar or nonpolar thermoplastic films that containing hybrid cellulose-copper nanoparticles. It is reported that foodborne pathogen could be divided into two main groups of Gram-negative and Gram positive 
bacteria. Based on the reports by others, Gram-negative and Gram-positive bacteria have different sensitivity to copper due to their different cell membrane structure (Ruparelia et al. 2008; Azam et al. 2012).

In chapter 4 and chapter 5, although PVA composite films containing either hybrid $\mathrm{CMC} /$ copper nanoparticles or hybrid TNFC/copper nanoparticles showed strong antimicrobial activities against E.coli DH $5 \alpha$. Based on antimicrobial and copper ion release studies, we also proposed that the bacterial reduction on PVA/copper nanoparticles films are likely to be the result of a combination of different factors, the antimicrobial activities of PVA/cellulose-copper nanoparticles films not only depend on the copper ion release but also on the oxidation states of copper within the films. The exact antimicrobial mechanism of PVA/hybrid cellulose-copper nanoparticles composite films is not fully understood. Based on the literature review, three of the most pertinent determinants of antimicrobial efficacy is (1) copper nanoparticles, (2) copper ion release from the copper nanoparticles and (3) copper ion-induced ROS (Macomber and Imlay 2009; Santo et al. 2010; Mallick et al. 2012; Chatterjee et al. 2014). I recommend that future work are needed to further determine the correlation between antimicrobial properties and ROS that has been considered as main toxicity of copper, and investigate the damage of bacterial cells after exposure to PVA composite so as to provide more comprehensive knowledge about the precise antimicrobial mechanisms of copper nanoparticles on the films.

In the chapter 6 , the tensile strength were found to be compromised after the incorporation of FD hybrid materials into the nonpolar thermoplastic resins because the agglomerations of FD hybrid materials existed in the matrices, the copper ion leaching from PLA is not insufficient to inactivate E.coli because PLA is hydrophobic so that water cannot easily diffuse into matrix and trigger the copper ion leaching. I recommend in the future SD hybrid materials should be considered to be incorporated into the matrices because their much smaller size of particles when compared to FD hybrid materials, in the meantime, increasing the copper contents in the matrix also should be considered in order to make more copper ion release from the films. 
On the synthesis of copper nanoparticles on cellulose templates, we also recommend to further investigate the effect of other factors such as $\mathrm{pH}$, reducing agent, carboxylic group concentration on the templates.

\subsection{References}

Azam, A., Ahmed, A. S., Oves, M., Khan, M. S., Habib, S. S., \& Memic, A. (2012). Antimicrobial activity of metal oxide nanoparticles against Gram-positive and Gramnegative bacteria: a comparative study. International Journal of Nanomedicine, 7, 6003.

Chatterjee, A. K., Chakraborty, R., \& Basu, T. (2014). Mechanism of antibacterial activity of copper nanoparticles. Nanotechnology, 25(13), 135101.

Macomber, L., \& Imlay, J. A. (2009). The iron-sulfur clusters of dehydratases are primary intracellular targets of copper toxicity. Proceedings of the National Academy of Sciences, 106(20), 8344-8349.

Mallick, S., Sharma, S., Banerjee, M., Ghosh, S. S., Chattopadhyay, A., \& Paul, A. (2012). Iodine-stabilized $\mathrm{Cu}$ nanoparticle chitosan composite for antibacterial applications. ACS Applied Materials \& Interfaces, 4(3), 1313-1323.

Ruparelia, J. P., Chatterjee, A. K., Duttagupta, S. P., \& Mukherji, S. (2008). Strain specificity in antimicrobial activity of silver and copper nanoparticles. Acta Biomaterialia, 4(3), 707-716.

Santo, C. E., Morais, P. V., \& Grass, G. (2010). Isolation and characterization of bacteria resistant to metallic copper surfaces. Applied and Environmental Microbiology, 76(5), $1341-1348$ 